Florida International University FIU Digital Commons

6-28-2012

\title{
Social and Environmental Regulation of Signal Plasticity and Signal Reliability in the Electric Fish Brachyhypopomus gauderio
}

Sat Gavassa Becerra

Florida International University, sgava001@fiu.edu

DOI: $10.25148 /$ etd.FI12080606

Follow this and additional works at: https://digitalcommons.fiu.edu/etd

\section{Recommended Citation}

Gavassa Becerra, Sat, "Social and Environmental Regulation of Signal Plasticity and Signal Reliability in the Electric Fish Brachyhypopomus gauderio" (2012). FIU Electronic Theses and Dissertations. 687.

https://digitalcommons.fiu.edu/etd/687 


\section{FLORIDA INTERNATIONAL UNIVERSITY}

Miami, Florida

SOCIAL AND ENVIRONMENTAL REGULATION OF

SIGNAL PLASTICITY AND SIGNAL RELIABILITY

IN THE ELECTRIC FISH BRACHYHYPOPOMUS GAUDERIO

A dissertation submitted in partial fulfillment of

the requirements for the degree of

DOCTOR OF PHILOSOPHY

in

BIOLOGY

by

Sat Gavassa Becerra

2012 
To: Dean Kenneth G. Furton

College of Arts and Sciences

This dissertation, written by Sat Gavassa Becerra, and entitled Social and Environmental Regulation of Signal Plasticity and Signal Reliability in the Electric Fish Brachyhypopomus gauderio, having been approved in respect to style and intellectual content, is referred to you for judgment.

We have read this dissertation and recommend that it be approved.

Zhenmin Chen

William Crampton

Fernando Noriega

Michael Heithaus

Philip K. Stoddard, Major Professor

Date of Defense: June 28, 2012

The dissertation of Sat Gavassa Becerra is approved.

\begin{tabular}{r} 
Dean Kenneth G. Furton \\
College of Arts and Sciences \\
\hline Dean Lakshmi N. Reddi \\
University Graduate School
\end{tabular}

Florida International University, 2012 


\section{DEDICATION}

This dissertation is dedicated to the 155 fish whom made the ultimate commitment to my research and unwillingly gave their lives to make this dissertation possible. It is my pledge to them that their sacrifice did not go to waste. 


\section{ACKNOWLEDGMENTS}

I want to thank the members of my dissertation committee, Dr. Zhenmin Chen, Dr. William G. R. Crampton, Dr. Michael Heithaus, Dr. Fernando Noriega, and Dr. Philip K. Stoddard for their dedication, thoughtful discussions and advice on my dissertation research and my career. I also want to thank other professors outside my committee whom gave me guidance and encouragement: Dr. Maureen Donnelly and Dr. Lidia Kos. I want to especially thank my Major Professor, Dr. Philip K. Stoddard, for his unmitigated support and always finding a creative way to enrich my approach to science. Even when we disagree, he is patient and kind, and he always sees the bright side of things. Having him as a mentor helped me grow academically, intellectually and personally.

I also want to thank Dr. Ana C. Silva, who opened the doors of her lab, her friendship and her family to me. Without her collaboration this dissertation would not have been possible. I am tremendously thankful to her for her commitment to my research, and I am enormously thankful for having had the opportunity to work with her. I would also like to give a special thank you to the members of Dr. Ana Silva's lab and their family members who worked arduously helping me collect the data for this dissertation. My methodological design required what my advisor called "tour-the-force",

which wouldn't have been possible without the human power and expertise of Rossana Perrone, Lucia Zubizarreta, Gervasio Batista, Tabaré de los Campos, Darío Colacce, Paula Pouso, and Alvaro Cabana.

I also want to give special thanks to the members of the Stoddard lab, past and present, Enrique Machado, Sam Rhodes, Vanessa Trujillo, Christine Muñoz, and Vielka 
Salazar for assistance in the lab and manuscript revisions. I would like to thank the undergraduates who helped me collect part of the data for this dissertation: Emmanuel Gonzalez, James Roach, Juan Molina and Pedro E. Perez. I wish to thank Carol Curtis for keeping the lab's boat afloat while I focused on my research. I want to thank Anya Goldina for allocating time out of her busy schedule to train me and discuss my research, but most importantly I'm grateful for her unconditional friendship and support.

I want to give very special thanks to my parents, Ilda Becerra and Virgilio Gavassa, for their support in my academic and personal development. I also want to give special thanks to my fiancé, Justin Campbell, who gave me the strength to keep on going and always keep pushing.

Finally I would like to thank my generous funding sources. My work was supported by FIU University Graduate School's Dissertation Evidence Acquisition Fellowship and Dissertation Year Fellowship. I also received funding from Tinker Field Research Grants (LACC at FIU), Judith Evans Scholarship for travel, Animal Behavior Society Research Award, and several travel and research awards from the Graduate Student Association at FIU. I also received financial support from the NSF grant IOS 0956603 to Dr. Philip K. Stoddard. Part of this dissertation has already been published elsewhere. I obtained Copyright permissions for its reuse here (licenses: 2895650303849, 2895641209577, and 2900191064037). 


\author{
ABSTRACT OF THE DISSERTATION \\ SOCIAL AND ENVIRONMENTAL \\ REGULATION OF SIGNAL PLASTICITY AND SIGNAL RELIABILITY \\ IN THE ELECTRIC FISH BRACHYHYPOPOMUS GAUDERIO \\ by
}

Sat Gavassa Becerra

Florida International University, 2012

Miami, Florida

Professor Philip K. Stoddard, Major Professor

The balance between the costs and benefits of conspicuous signals ensures that the expression of those signals is related to the quality of the bearer. Plastic signals could enable males to maximize conspicuous traits to impress mates and competitors, but reduce the expression of those traits to minimize signaling costs, potentially compromising the information conveyed by the signals.

I investigated the effect of signal enhancement on the information coded by the biphasic electric signal pulse of the gymnotiform fish Brachyhypopomus gauderio. Increases in population density drive males to enhance the amplitude of their signals. I found that signal amplitude enhancement improves the information about the signaler's size. Furthermore, I found that the elongation of the signal's second phase conveys information about androgen levels in both sexes, gonad size in males and estrogen levels in females. Androgens link the duration of the signal's second phase to other androgenmediated traits making the signal an honest indicator of reproductive state and aggressive motivation. 
Signal amplitude enhancement facilitates the assessment of the signaler's resource holding potential, important for male-male interactions, while signal duration provides information about aggressive motivation to same-sex competitors and reproductive state to the opposite sex. Moreover, I found that female signals also change in accordance to the social environment. Females also increase the amplitude of their signal when population density increases and elongate the duration of their signal's second phase when the sex ratio becomes female-biased. Indicating that some degree of sexual selection operates in females.

I studied whether male B. gauderio use signal plasticity to reduce the cost of reproductive signaling when energy is limited. Surprisingly, I found that food limitation promotes the investment in reproduction manifested as signal enhancement and elevated androgen levels. The short lifespan and single breeding season of B. gauderio diminishes the advantage of energy savings and gives priority to sustaining reproduction. I conclude that the electric signal of $B$. gauderio provides reliable information about the signaler, the quality of this information is reinforced rather than degraded with signal enhancement. 


\section{TABLE OF CONTENTS}

CHAPTER

PAGE

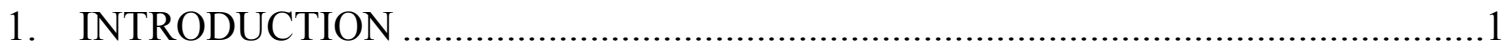

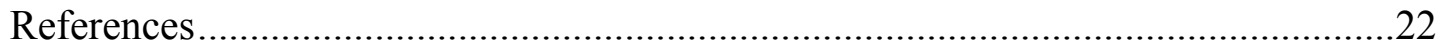

2. SIGNAL MODULATION AS A MECHANISM

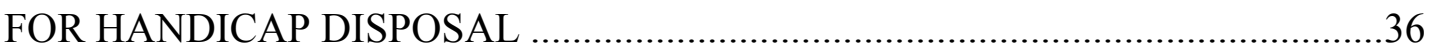

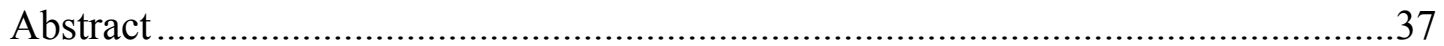

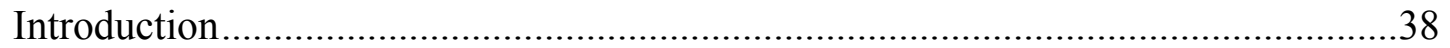

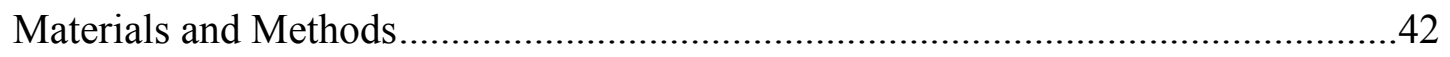

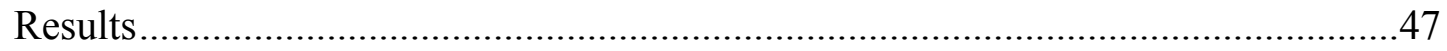

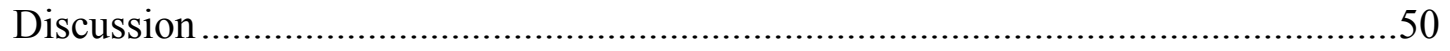

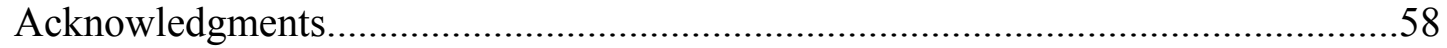

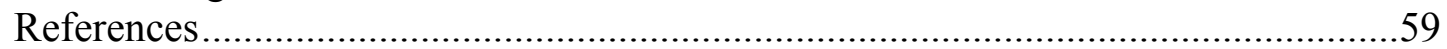

3. TIGHT HORMONAL PHENOTYPIC INTEGRATION ENSURES HONESTY OF THE ELECTRIC SIGNAL OF MALE AND FEMALE BRACHYHYPOPOMUS

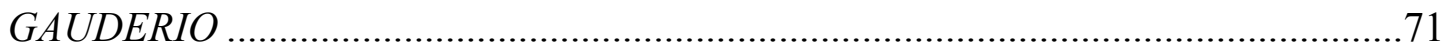

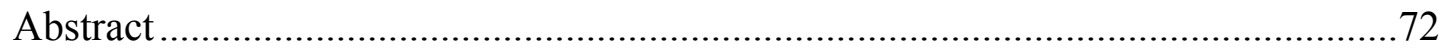

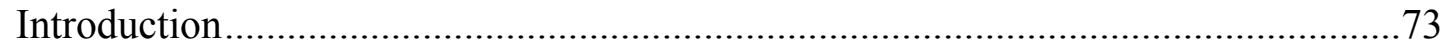

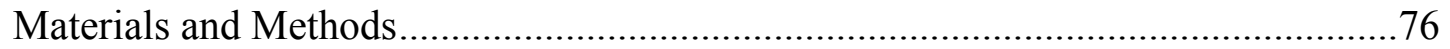

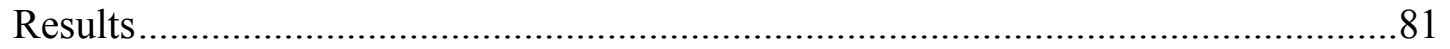

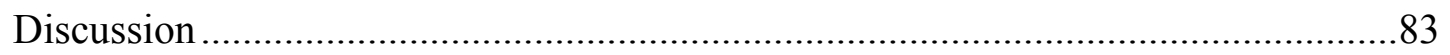

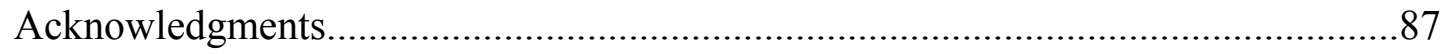

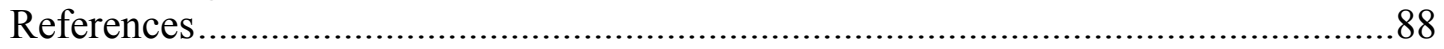

4. SOCIAL COMPETITION MASCULINIZES THE COMMUNICATION SIGNALS

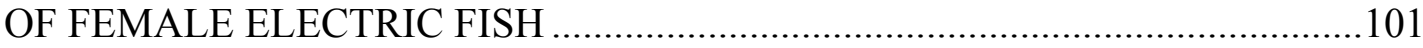

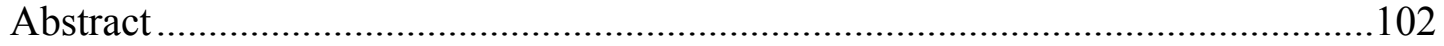

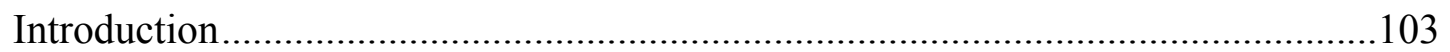

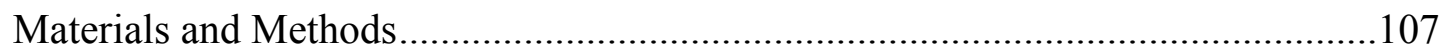

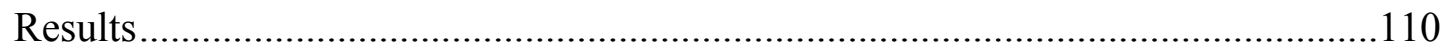

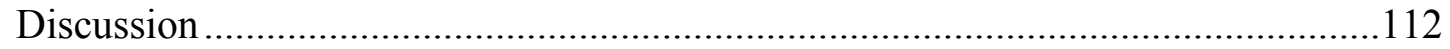

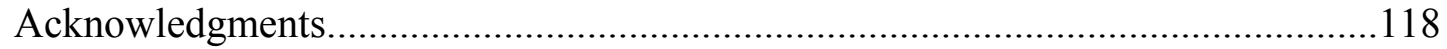

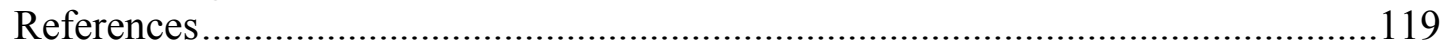

5. FOOD RESTRICTIONS PROMOTES SIGNAL ENHANCEMENT IN RESPONSE TO SOCIAL CHALLENGE IN A SHORT-LIVED ELECTRIC FISH ...................131

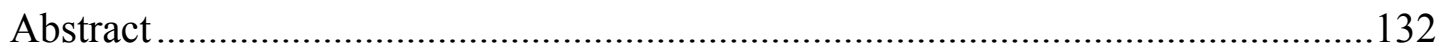

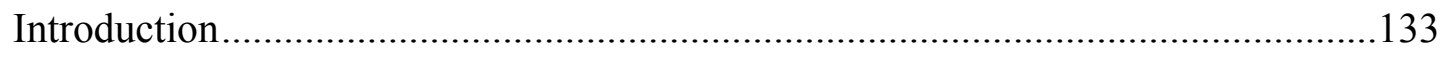

Materials and Methods...............................................................................137

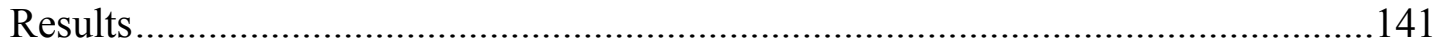

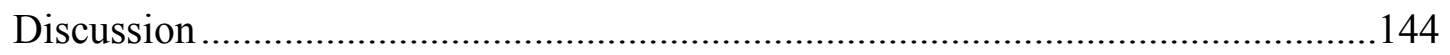




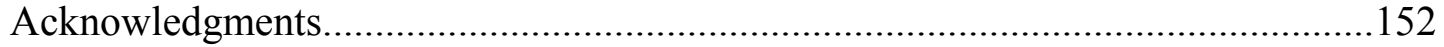

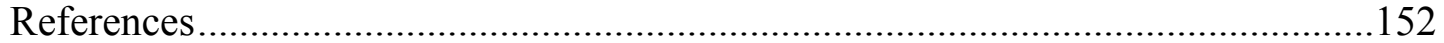

6. SOCIAL REGULATION OF ELECTRIC SIGNAL PLASTICITY IN MALE

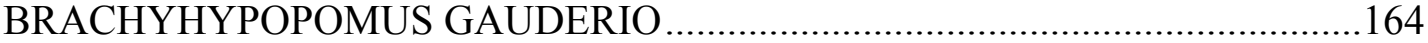

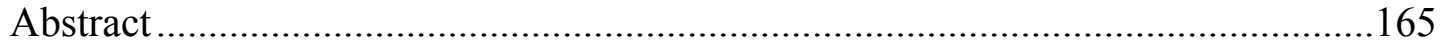

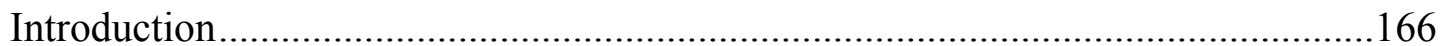

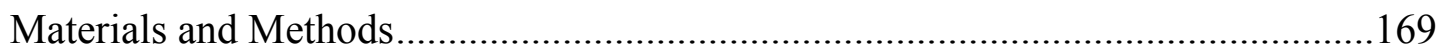

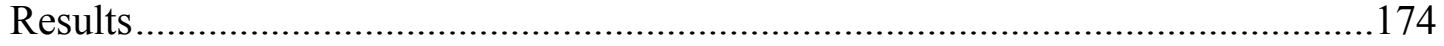

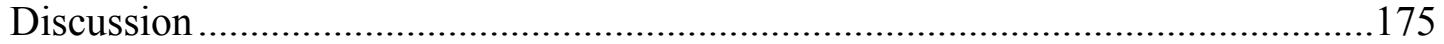

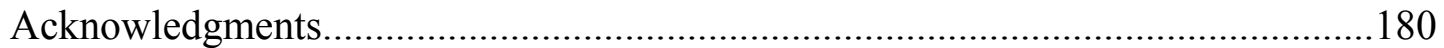

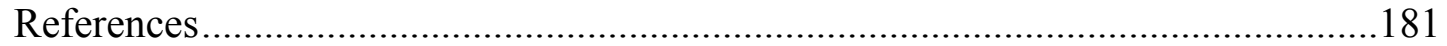

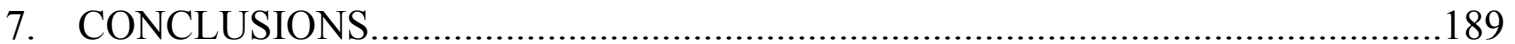

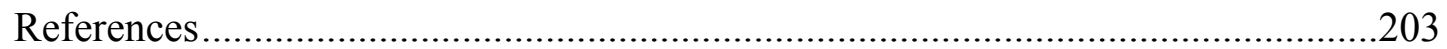

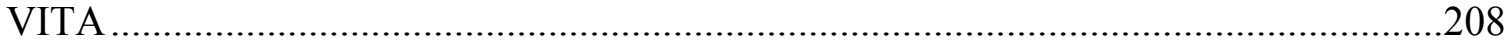




\section{LIST OF FIGURES}

FIGURE

PAGE

Chapter 1

Figure 1.1. Graphical model of signaling equilibrium.............................................32

Figure 1.2. Reproductive opportunity model.........................................................33

Figure 1.3. Cortisol levels during the breeding season $v$ s. reproduction intensity ............34

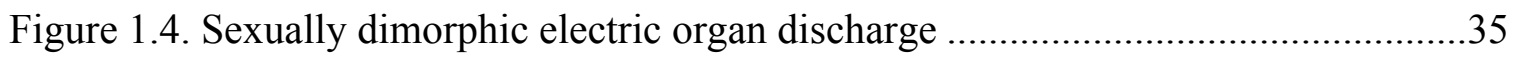

Chapter 2

Figure 2.1. Relationship between EOD amplitude and body length.............................66

Figure 2.2. Amplitude of the EOD in relation to population density ............................67

Figure 2.3. Relationship between signal amplitude, body length and population density

Figure 2.4. Reliability of EOD amplitude as a predictor of body length at different population densities

Figure 2.5. Change in EOD amplitude in relation to EOD amplitude predicted during low competition and in relation to body length .70

Chapter 3

Figure 3.1. Phenotypic integration and signal reliability .94

Figure 3.2. The biphasic EOD of B. gauderio .95

Figure 3.3. Androgen levels and EOD t $t_{\mathrm{P} 2}$ in males and females .95

Figure 3.4. Testosterone levels are related to 11-ketotestosterone in both sexes and estradiol in females. .96

Figure 3.5. Relationship between androgen levels and EOD $t_{\mathrm{P} 2}$ parameter .97

Figure 3.6. The EOD as an indicator of other androgen-mediated traits........ .98

Figure 3.7. Relationships that arise via phenotypic integration 


\section{Chapter 4}

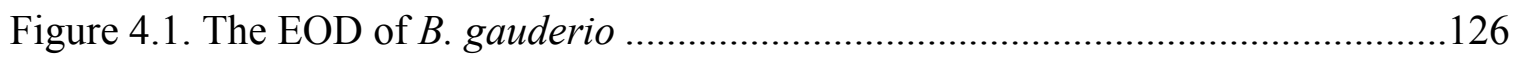

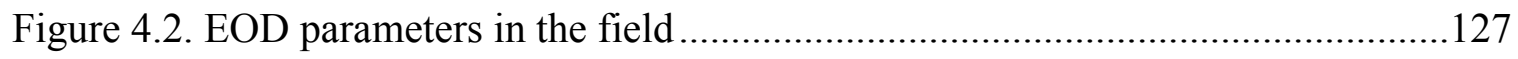

Figure 4.3. Steroid levels in relation to adult sex-ratio

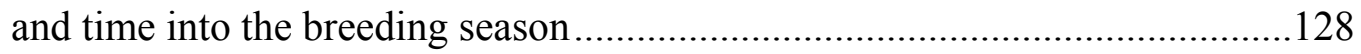

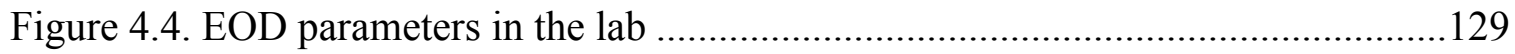

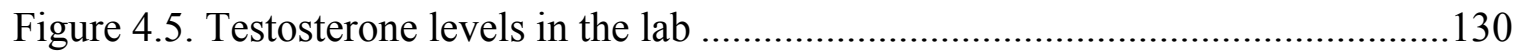

\section{Chapter 5}

Figure 5.1. The EOD of $B$. gauderio is a biphasic pulse 157

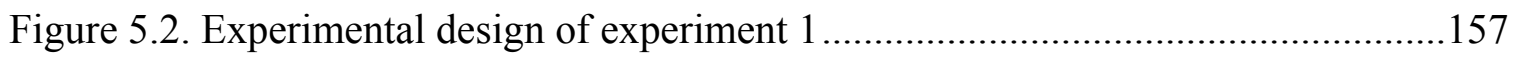

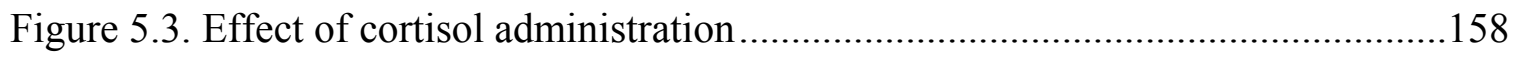

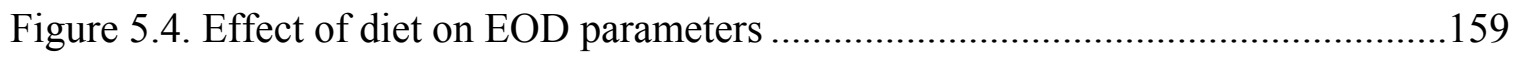

Figure 5.5. Effect of diet and social treatment on EOD parameter..............................160

Figure 5.6. Hormone profile for each treatment group

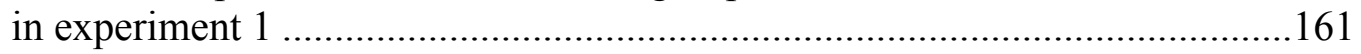

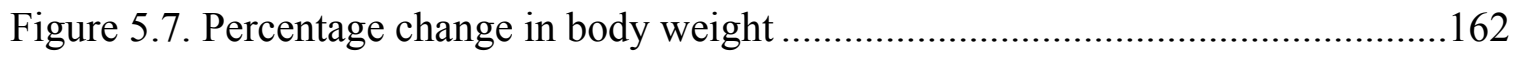

Figure 5.8. Change in the average of worms eaten daily ......................................... 163

\section{Chapter 6}

Figure 6.1. The electric organ discharge of B. gauderio .............................................186

Figure 6.2. Dissecting signal enhancement mechanisms .........................................186

Figure 6.3. Percentage change in EOD parameters in experiment 1 ............................187

Figure 6.4. Percentage change in EOD parameters in experiment 2 .......................... 188

Chapter 7

Figure 7.1. Cortisol vs. EOD amplitude corrected for body length ............................207 


\section{LIST OF TABLES}

TABLE

PAGE

\section{Chapter 1}

Table 1.1. List of marsupial species.

Chapter 2

Table 2.1. Log-Log relationship among sex steroid levels and steroid-mediated traits

Supplementary table 2.1. Equations for the regression analyses of the traits studied .......93

\section{Chapter 4}

Table 4.1. Summary of changes in EOD parameters and testosterone levels.

\section{Chapter 6}

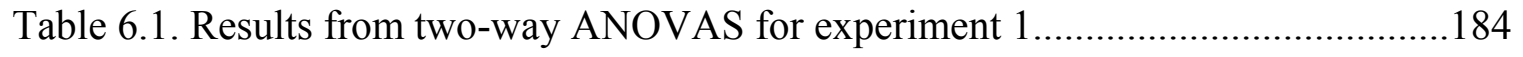

Table 6.2. Results from one-way ANOVAS for experiment 2 ...................................185 


\section{LIST OF ABBREVIATIONS}

$11-\mathrm{KT}$

$\alpha-\mathrm{MSH}$

ACTH

$\mathrm{AL}$

ANOVA

AP

ASR

CPUE

DHT

E2

EDTA

EHV

EOD

F

FR

GSI

HPA

HPI

HPG

IACUC

OSR

RHP 11-ketotestosterone

alpha-melanocyte stimulating hormone

adrenocorticotropic hormone

ad libitum

analysis of variance

action potential

adult sex-ratio

capture per unit of effort

$5 \alpha$-dihydrotestosterone

estradiol

ethylenediaminetetraacetic acid

energetics-hormone vocalization model

electric organ discharge

cortisol

food restricted

gonadosomatic index

hypothalamus-pituitary-adrenal axis

hypothalamus-pituitary-interrenal axis

hypothalamus-pituitary-gonadal

institutional animal care and use committee

operational sex-ratio

resource holding potential 
Pn

PPn

SPPn

SPSS

SEM

$\mathrm{T}$

$t_{P 2}$ pacemaker nucleus

prepacemaker nucleus

sublemniscal prepacemaker nucleus

statistical package for the social sciences

standard error of the mean

testosterone,

time constant of the repolarization of the second phase of the electric organ discharge 
CHAPTER 1

INTRODUCTION 
The sensory drive model of sexually selected display signals views organisms as trapped amid the trade-offs of attracting mates and the physiological and environmental costs of signaling (Otte, 1974; Endler, 1992; Maynard Smith and Harper, 1995). The costs of sexual signaling impose an extra energetic load on males that results in stress because of energy imbalance. However, species and individuals differ in their vulnerability to stress and in the way they cope with stress (Creel et al., 1996; Boonstra and McColl, 2000; Sapolsky, 2000; McEwen, 2002; Wingfield and Sapolsky, 2003; Wingfield, 2005; Breuner et al., 2008; Romero et al., 2009). Species capable of behavioral plasticity might have an added advantage that could enable them to circumvent reproductive trade-offs by adjusting their behavior to the supply and demands of the current environment (West-Eberhard, 1989; Tomkins et al., 2005). In the context of sexual communication, animals produce signals that can be used by their competitors to learn about the fighting ability of the sender or by potential mates to infer the quality of the signaler (Andersson, 1994). Plastic signals could allow signalers to transiently escape environmental and physiological constraints of signaling and portray themselves as being of better quality than they actually are. Conversely, signal plasticity could help signalers minimize costs by reducing signaling intensity when return on signal investment is low. I studied how a species with a plastic communication signal would use that plasticity to navigate around reproductive trade-offs and the consequences that plasticity could have on the honesty of the information conveyed by the signal. 


\section{$\underline{\text { Signal plasticity and signal honesty }}$}

Students of behavior have pondered why animal signals remain honest in competitive contexts despite the apparent temptations and benefits of cheating, lying, bluffing, or exaggerating (Dawkins and Krebs, 1978). Nonetheless, widespread dishonesty will turn the signals meaningless and receivers will ultimately ignore them (Otte, 1974). Thus, scholars have postulated numerous models for the persistence of honest communication in the face of pressures to cheat. According to the Handicap Principle, signal honesty is guaranteed when the advertising signal meets the following three conditions: 1) The expression of the signal depends on a sexually selected phenotypic trait of the signaler, 2) signals are expensive to produce or maintain and, 3) the costs of signaling rises faster than its benefits (Zahavi, 1975; Zahavi, 1977; Grafen, 1990). An advertisement signal can be referred to as an "index" if its properties depend physically on the magnitude of a physical trait of the signaler, such that the signaler has no control over the magnitude of the signal and cannot exaggerate (Maynard Smith and Harper, 1995). Despite its cost, honest communication is evolutionary stable when it provides overall selective benefits to both signalers and receivers (Searcy and Nowicki, 2005).

A corollary puzzle then, is how deception can persist in communication systems if deception is not evolutionarily stable. Posterior models found that communication prevails when deceit is limited to the following scenarios: some signalers face reduced cost of signaling compared with other signalers (Grafen, 1990), aspects of the phenotype mask the genetic quality being advertised (Kokko, 1997), or when the benefit of signaling 
at high intensity for low quality signalers outweighs the cost of increased signaling (Adams and Mesterton-Gibbons, 1995; Candolin, 1999). Grafen and Johnstone (1993) proposed that deception can be stable if receiver's response to the signal is adaptive on average. In the latter case, dishonesty persists when the receiver's cost of failing to respond to an honest signal is greater than the cost of responding to a dishonest signal (Wiley, 1994).

Behaviorally plastic communication signals could provide a signaler with multiple advantages. For instance, plastic signals can be enhanced in the presence of potential mates and competitors but they can be decreased to reduce conspicuousness when predators are present or to reduce physiological costs (Nur and Hasson, 1984). Plastic signals could better portray current state of the individual, responding to immediate changes in the hormonal profile of the individual (Parker et al., 2002; McGraw et al., 2005; McGlothlin et al., 2008; Lindsay et al., 2009).

Wagner (1992) proposed three hypotheses to explain what could happen to the signal honesty when the signaler increases the magnitude of the signal. 1) The "signal of size" hypothesis proposes that increasing the signal's magnitude makes the signal more honest, in other words, makes the signal a better predictor of body size. In this case, signalers are performing near their physiological maximum resulting in an improvement of signal reliability. The "signal of size" hypothesis could explain why, in some species, the honesty of the signal increases in the presence of mates (Gautier et al., 2008) or competitors (Howard and Young, 1998), when one would otherwise expect dishonesty because of bluffing. 2) The "signal of size-independent fighting ability" hypothesis states 
that the increase in signal output is not related to size but to an additional attribute of the signaler such as fighting ability, motivation or, energetic condition. For example, fiddler crabs (Kim et al., 2008) and the barking tree frog (Murphy, 1994) increase signaling output when they have more food available. Although the fundamental frequency of cricket frogs' song is normally related to the size of the signaler, males change the frequency of their calls during contests with other males without relation to their body size (Bee and Perrill, 1996; Burmeister et al., 1999). These changes in frequency are not related to body size but instead provide information about the aggressive intent of the signaler (Burmeister et al., 2002). 3) The "dishonest signal of size" hypothesis proposes that increasing signaling output exaggerates the apparent size of the signaler, thus conveying false information about the signaler. This scenario may occur when the potential benefits of signaling surpass the cost of signal exaggeration (Adams and Mesterton-Gibbons, 1995). For example, individuals in their last opportunity to reproduce may expend more energy in advertising than expected for their condition (Candolin, 2000a; Proulx et al., 2002). On the other hand, signal honesty can increase via socially imposed costs on low quality individuals that would otherwise bluff as intrasexual competition increases (Rohwer and Ewald, 1981; Candolin, 2000c; Candolin, 2000b; Tibbetts and Dale, 2004).

Even when signal exaggeration leads to a decrease in the honesty of the information conveyed by a particular signal parameter, other parameters can emerge as honest indicators of quality. For instance, the length of the vocal tract determines the fundamental frequency of acoustic signals, primarily the distance from the larynx to the 
external environment (Fitch and Hauser, 2002). Thus, fundamental frequency should be an honest index of size, but animals could potentially exaggerate their size by elongating their vocal tract. Some mammals do, in fact, elongate their vocal tract by positioning the larynx deeper in the body, closer to the chest, while some birds elongate their vocal tract by elongating their trachea (Charlton et al.; Fitch, 1999; Fitch and Hauser, 2002). The elongation of the vocal tract disrupts the relationship between the call's dominant frequency and body size. However, the larynx cannot be lowered beyond the sternum. Resonance in the acoustic path from the larynx to the external environment reinforces particular spectral frequencies, producing formant peaks. As a result, the active lowering of the larynx reinforces the relationship between body size and formant spacing, enhancing the honesty of formant frequency as an indicator of body size (Reby and McComb, 2003).

\section{$\underline{\text { Signal plasticity and cost savings }}$}

I propose a model in which signalers benefit from producing lower quality signals (i.e., lower intensity) in order to reduce signaling costs. Reducing the quality of an expensive signal makes it a "disposable handicap". The proposition may sound counterintuitive, since signaling benefits increase with signal intensity, thus signalers should always signal at their maximum capacity. However, the relationship between signal benefits and signal intensity is context-dependent as the value of the resource changes. Under high competition, resources are scarce, thus their value increases. Consequently, the benefit from signaling to defend or gain access to the resource increases while the cost function of signaling remains unchanged (Fig. 1). As competition increases, signal 
intensity will increase until all individuals have regained their handicap and the signal is at its highest honesty. This model follows a similar rationale to Johnstone's (1997) model for "signals of need" (e.g., nestling begging calls) in that the equilibrium of signaling intensity is reached when the difference between signal cost and benefit is the greatest. What is different from Johnstone's (1997) model is that the benefit curve increases with competition, therefore the equilibrium point changes for the entire population and not just one individual.

On the other hand, for signals that increase predation risk, competition should reduce the cost of signaling when high density of signalers dilutes predation risk. In this way, competition frees signals to increase further in intensity or complexity (Ryan et al., 1982; Ryan, 1985). As long as the shape of the cost function remains unchanged, the cost-benefit equilibrium of signaling will still increase with competition (Fig. 1).

Of course receivers will benefit if competition results in transmission of more accurate information. As predicted by Wiley's (1994) "adaptive gullibility hypothesis"; in low competition, the cost of incorrectly rejecting an honest signal should outweigh the cost of responding to a dishonest signal, while the opposite should be true for high competition when the cost of believing a dishonest signal should outweigh the cost of disregarding an honest signal. For example, in low competition the cost of finding a new territory should be lower than the cost of engaging in an agonistic encounter with a territory holder who may be of better quality.

Despite these models, the ultimate question about the origin of signal plasticity remains unanswered. Did plasticity arise as a mechanism to exaggerate the signaler's condition until it reached a physiological ceiling causing honesty to emerge from 
exaggeration? Or did signalers increase their signals progressively until they reached the physiological limit and then acquired the plasticity to reduce signal intensity and its associated costs, the disposable handicap model? Signal modulation mechanisms that may have initially evolved under the selective advantage of exaggerating the signaler's quality could be maintained by the advantages of energy conservation under low competition, i.e., a disposable handicap. Conversely, energetic savings of handicap disposal could have been the original driver of signal modulation ability just as easily as exaggeration of perceived body size. Under this reversed scenario, competition could have driven the evolution of expensive signals, followed by the advantage of modulating the signal to save energy in during reduced competition.

I tested the assumptions of the disposable handicap model in Chapter 2, looking at the effect of competition and signal enhancement on the honesty of the signal in a species known to increase the intensity of its signal during social competition. Chapter 3, I tested whether other aspects of the signal could convey size-independent information such as reproductive condition and motivational state. In Chapter 4, I tested whether females were also capable of adjusting their signaling output to competition intensity, just as males do.

\section{Cost savings and life-history}

The advantages of signal plasticity to save on signaling cost should depend on the life-history of the species. The energetic expense of reproduction typically entails reconfiguration of the individual's energy budget. In response to an energy imbalance, iteroparous species favor investment in survival over investment on reproduction. On the 
other hand, semelparous species favor investment on reproduction over survival (Williams, 1966). In vertebrates, glucocorticoids (GCs) fulfill an essential role in energy management, typically diverting energy supply away from reproductive behavior during a crisis to favor survival. However, energetic reallocation for reproduction is particularly extreme in semelparous species, to the extent that some species may use GCs to release the body resources needed for reproduction. Semelparous vertebrates are typified by a seasonal rise in GC levels, as they divert energy from somatic maintenance into reproduction, (McEwen and Wingfield, 2003; Wingfield and Sapolsky, 2003).

Nonetheless, the rise in GCs depends on the intensity of investment in reproduction, which is inversely proportional to the duration of the breeding season. Semelparous species with short breeding seasons of few days or weeks show a particularly sharp rise in GC levels concurrent with the onset of reproduction (McDonald et al., 1981; Boonstra and McColl, 2000; Carruth et al., 2000; Barry et al., 2001). However, other semelparous species with longer breeding seasons (a few months) do not increase GC levels during the breeding season (Oakwood et al., 2001). I hypothesized that experimental shortening of the breeding season should cause energy to be reallocated away from survival and into reproduction and thus increasing intensity of reproductive signals. Likewise, whether or not GCs rise precipitously in semelparous species depends on the length of the breeding season itself. Semelparous species with a short breeding season are typically capital breeders, investing all their resources into a single bout of reproduction (Boonstra et al., 2001; Wingfield and Sapolsky, 2003; Boonstra, 2005). On the other hand, I predict that semelparous species with a longer breeding season should be more conservative with their energetic allocation using current income to help fuel reproduction while preventing 
a rise in GCs levels in order to endure throughout the breeding season. I questioned whether semelparous species with longer breeding seasons have mechanisms in place to prevent the rise in GCs and therefore reduce the detrimental effect of GCs on survival, more resembling the GC suppression of iteroparous species.

Sterling and Eyer (1988) coined the term allostasis to describe the physiological adaptations an organism undertakes to maintain internal stability through change. Physiological mediators orchestrate the physiological processes necessary to maintain homeostasis. These mediators include catecholamines, cytokines, and GCs, among others. McEwen $(1998 ; 2000 ; 2002)$ further developed the Allostasis Model to explain how the over-activation of allostatic systems in humans can lead to disease. The concept of allostasis was extended to model how various animals adapt to predictable and nonpredictable changes that affect their energetic balance (McEwen and Wingfield, 2003). Recently, the Reactive Scope Model extended the Allostasis Model by incorporating aspects of the life history of the animal and broadening the model's inputs beyond GCs and outputs beyond energy balance to include multiple physiological processes (Romero et al., 2009).

To provide a framework for exploring the role of GCs in reproduction and social signals Dr. Philip K. Stoddard and I advanced the "Reproductive Opportunity Model" (Stoddard \& Gavassa, unpublished). This model casts existing models of physiological regulation (Allostasis and Reactive Scope) against an axis representing opportunity for future reproduction. This axis derives from life history theory of iteroparity \& 
semelparity (Charnov and Schaffer, 1973), a connection proposed recently by Boonstra (2005).

Elements of the model: The horizontal axis (Fig. 2) represents a continuum of opportunity for reproduction remaining in an individual's life, or the intensity of reproduction. Animals with fewer opportunities to reproduce in the future should breed more intensely. This axis can be applied within the life history of the individual (e.g., young vs. old), between the sexes, or across taxa (iteroparous vs. semelparous). The vertical axis includes any physiological mediators of metabolism writ large, such as GCs levels. Energy consumption could be the regulated output, as in the Allostasis Model, but so could protein synthesis. Following from the Allostasis/Reactive Scope Models, the predictive homeostasis zone (gray band) describes the circulating range of the mediator evoked by circadian rhythms and normal activity. Animals may drop down into the under-performance zone, perhaps with a cost to fitness but not to survival (Romero et al., 2009). Homeostatic failure occurs when the animal cannot maintain the minimum output needed to sustain life. Homeostatic overload represents an unsustainable output, in which output exceeds input, and somatic systems are expected to fail over time. Deleterious physiological effects accumulate when animals remain in homeostatic overload (McEwen, 1998; McEwen, 2000; McEwen and Wingfield, 2003; Romero et al., 2009). In many cases some headroom exists between predictive homeostasis and homeostatic overload, an area called "reactive homeostasis" (Romero et al., 2009). However, the dark gray triangle, where predictive homeostasis overlaps homeostatic overload, seems like a 
contradiction because it describes a situation where failure is normal and expected (Stoddard \& Gavassa, unpublished).

Given the difficulties of estimating the opportunity for reproduction across the entire range of iteroparity and semelparity, I chose to focus on semelparous species with varying durations of the breeding season. Using only semelparous species, I can estimate the intensity of reproduction as a fraction of the duration of the breeding season to lifespan. Highly elevated glucocorticoids levels have been observed in semelparous species, for example sockeye salmon (Oncorhynchus nerka) (Carruth et al., 2000; Barry et al., 2001), arctic ground squirrels (Spermophilus parryii plesius) (Boonstra and McColl, 2000; Boonstra et al., 2001), and marsupial species from the genera Antechinus and Phascogale (Bradley et al., 1980; McDonald et al., 1981; Bradley, 1997). All of these species have a very short breeding season lasting from a few days (sockeye salmon) to a couple weeks (arctic ground squirrels and marsupials). However, the marsupial Dasyurus hallucatus shows no rise in cortisol levels during the breeding season (Oakwood et al., 2001), even though its life-history is very similar to Antechinus and Phascogale species, where all males die after a single breeding season. However, the breeding season of Dasyurus hallucatus lasts 3-4 months, much longer than the breeding season of Antechinus and Phascogale species (2-3 weeks) (Bradley et al., 1980; McDonald et al., 1981; Bradley, 1997; Oakwood et al., 2001).

I tested the assumptions of the model using a group of marsupials in which the males show a characteristic die-off after the breeding season. This is an ideal group in which to study how GC levels vary with intensity of reproduction because the species in 
this group are closely related, show great variation in the intensity of reproduction and have well documented breeding season and hormonal profiles. I found that published GC levels during the breeding season correlate almost perfectly with the intensity of reproduction (the ratio of the breeding days to life-span). Species in which breeding season lasts just a few days showed the highest levels of circulating GC during reproduction compared low GCs in species whose breeding season lasted a few months (Fig. $3 \&$ Table 1). To further test the predictions of the model, in Chapter 4 I studied the effect of GC on the reproductive signal of the electric fish Brachyhypopomus gauderio, a semelparous fish species with a relatively long breeding season (3-4 months) (Silva et al., 2003).

Electric fish are an exceptional model for studying behavioral flexibility linked to the neuroendocrine response.

I am interested in understanding the evolution of signal plasticity. In one hand, signal plasticity could offer great advantages to the signaler, allowing it to exaggerate its signal to mates and competitors and decrease it in the absence of conspecific receivers or in the presence of predators. However ideal this system from the signaler's standpoint, dishonest signaling is disadvantageous for receivers, who should be selected to ignore such signals. Thus, on first pass, signal plasticity would not seem to be an evolutionary stable strategy. For signal plasticity to remain evolutionary stable it must balance the benefits to the signaler and the cost to the receiver. First, plastic signals must retain at least some information useful to receivers; and second, plasticity should provide an advantage to signalers, such as reduction on signaling costs. To test this hypothesis, I 
need a species with a plastic communication signal, but which is easily measured and is easy to estimate the type of information and the quality of the information conveyed in its signals. The communication signal of the species should have measurable costs.

Signaling cost should be high enough to provide incentive for energy savings.

Electric fish offer great advantages for the study of animal behavior. The constant generation of EODs offers an exceptional opportunity to continuously monitor their behavior in a non-invasive manner. The ease of recording their behavior, the consistent hormonal and behavioral response to changes in the social environment, along with the tight correspondence of behavior to hormone administration, make electric fish unique models to study the interaction between the environment and endocrine state, and their effect on behavior.

Neotropical electric fish of the order Gymnotiformes are distributed from northern Argentina to southern Mexico, largely contributing to the icthyofauna diversity of South America (Crampton, 1996; Albert and Crampton, 2005). Gymnotiforms produce electric organ discharges (EOD) for electrolocation and presumably for communication as well. Their nocturnal habits and the murky waters they inhabit suggest the importance of the EOD for navigation and communication (Moller, 1995). I studied Brachyhypopomus gauderio, a species that inhabits Uruguay, Southern Brazil and Northern Argentina. $B$. gauderio reproduces throughout the austral summer in its natural habitat (Silva et al., 2003). Under field conditions, B. gauderio is an annual - only immature fish can be found during the austral winter, indicating that adults from the previous year have not survived the breeding season (Silva et al., 2002). The primary sex ratio is unity (A. Silva 
\& K. Dunlap, unpubl. data), but reproductive males disappear quickly from the population as the breeding season advances, leaving sex ratios of about 1:4 after one month of breeding effort (Miranda et al., 2008).

Although males and females generate electric signals, in some species the discharges are sexually dimorphic (Hopkins, 1981; Hagedorn and Carr, 1985; Hopkins et al., 1990; Franchina and Stoddard, 1998). Brachyhypopomus gauderio produces a biphasic sexually dimorphic EOD. At night, males discharge at higher rates than females and generate EODs with different waveforms (Bass and Hopkins, 1983; Hopkins et al., 1990; Hagedorn, 1995; Franchina and Stoddard, 1998; Franchina et al., 2001). The nocturnal male EOD has a higher amplitude and longer duration than the female EOD, increasing its energetic cost (Salazar and Stoddard, 2008) and predation risk (Stoddard, 1999; Stoddard, 2002b).

Some electric fish modulate their discharge rates, increasing the rates during courtship or agonistic interactions, and between daytime rest and nighttime activity (Bass and Hopkins, 1983; Zakon et al., 1991; Hagedorn, 1995; Franchina and Stoddard, 1998; Franchina et al., 2001; Dunlap, 2002; Silva et al., 2007). Brachyhypopomus gauderio enhances its EOD following a circadian rhythm: they reduce rate, amplitude and duration of the electric organ discharge during the day and increase them during the night when the fish are more active (Franchina and Stoddard, 1998; Franchina et al., 2001; Silva et al., 2007; Stoddard et al., 2007). Both, males and females follow this circadian pattern but it is enhanced in males (Fig. 4). The decline of the EOD during the day could be explained as a strategy to avoid predation during the day and reduce the cost of signal 
production (Stoddard, 1999; Stoddard, 2002b; Stoddard, 2002a; Salazar and Stoddard, 2008). Electric signals of males are costly handicaps; they are energetically expensive, consuming an average of $22 \%$ of the daily energetic budget (compared to $3 \%$ for females), and their energetic costs rise with the amplitude and duration of the EOD waveform (Salazar and Stoddard, 2008).

The male's further enhancement of his EOD at night seems to serve for attracting mates or deterring competing males. When given a choice, females prefer large males, which produce the most enhanced EODs (Curtis and Stoddard, 2003). Social isolation reduces the male's EOD, accompanied by drops in androgens and cortisol (Salazar and Stoddard, 2009). The EOD partially recovers minutes after the introduction of a conspecific to the tank, and fully recovers after three days, a pattern consistent with the intermediate-term effects of melanocortins and long-term effects of androgen administration (Franchina et al., 2001; Stoddard et al., 2006). The EOD recovers faster with the introduction of a male, especially if that male has a higher EOD amplitude than the focal male (Stoddard et al., 2006).

\section{Neuroendocrine control of the EOD}

The EOD is the result of the summation of simultaneous electric discharges of individual electrocytes, which compose the electric organ (EO). The EO of B. gauderio extends bilaterally from the back of the pectoral fin to the end of the caudal filament, extending along most of the fish's body. Each electrocyte is innervated on the posterior side by a spinal electromotor neuron driven by the medullary pacemaker nucleus (Pn). The Pn computes the input from the diencephalic prepacemaker nucleus (PPn) and the 
sublemniscal prepacemaker nucleus (SPPn) to set the pace of the action potentials (APs) traveling down in the electromotor neurons (rev. Lorenzo et al., 2006). The APs ultimately reach the electrocytes and initiate the EOD. In the electrocyte, an AP induces the movement of ions across the excitable membrane resulting in an electric discharge of an individual electrocyte.

At the cellular level, the EOD waveform depends on the geometry of the electrocyte and the kinetics of the ion channels in the excitable membranes of the electrocyte (Zakon et al., 1999). Brachyhypopomus gauderio generates a biphasic EOD, composed of a first, head-positive phase and a second, head-negative phase. The first phase occurs when all the electrocytes fire an action potential from the innervated posterior membrane of the electrocyte. This AP moves $\mathrm{Na}^{+}$for the posterior side to the electrocyte ions into the cell creating a positive current flowing towards the head. The second phase is generated by the electrocytes in the caudal half of the fish. Theses electrocytes fire a second action potential from the anterior non-innervated membrane (Stoddard and Markham, 2008). The flow of $\mathrm{Na}^{+}$ions into the cell from the anterior side creates a positive current towards the tail, resulting in a head-negative phase.

The EOD is controlled by two largely independent mechanisms. The discharge rate is controlled via synaptic connections, by the interaction of the SPPn and the PPn with the Pn. The EOD waveform is controlled via endocrine messengers acting at the electrocyte. Serotonin injections in vivo promote changes in the EOD waveform similar to those observed during social interactions (Stoddard et al., 2003). However, serotonin has no effect in the electrocyte, in vitro, which suggests that it acts centrally to mediate 
peripheral melanocortin release (Markham and Stoddard, 2005; Stoddard, 2006; Stoddard and Markham, 2008). The melanocortin hormones, adrenocorticotropic hormones (ACTH) and $\alpha$-melanocyte-stimulating hormone $(\alpha-\mathrm{MSH})$, enhance the waveform when applied in vivo and in vitro to the electrocyte. Furthermore, blocking melanocortin receptors diminishes the EOD amplitude and duration (Markham et al., 2009a). In another electric fish species, Sternopygus macrurus, melanocortins were shown to increase the amplitude of the EOD by increasing the trafficking of ion channels into the excitable membrane of the electrocyte (Markham et al., 2009b). These data corroborate the idea of melanocortins being pivotal for EOD waveform modulation. Androgens are also known to change the waveforms, by initiating transcription of ion channels subunits with altered kinetics of ion channels (Bass and Hopkins, 1983; Carlson et al., 2000; Bass and Zakon, 2005; Liu et al., 2008). Androgen implants masculinize the female EOD (Allee et al., 2009) and further extend the amplitude and duration of male EOD (Goldina et al., 2011). Moreover, androgens synergize with melanocortins by increasing the responsiveness of the EOD to melanocortin hormones (Allee et al., 2009; Goldina et al., 2011).

To explore hypotheses related to honest signaling (e.g., disposable handicap hypothesis), it helps greatly to know what information the signals can convey to receivers. The EOD of B. gauderio could serve as an indicator of body length since the amplitude of the signal physically depends on the length of the electric organ, which runs the length of the fish's body (Hopkins et al., 1990; Hopkins, 1999; Curtis and Stoddard, 2003). Moreover, body length is key for mate-choice and male-male interactions, since 
longer males are more attractive to females (Curtis and Stoddard, 2003) and more likely to win agonistic encounters (Salazar, 2009; Silva et al., 2010). Therefore, receivers should pay particular attention to any information about body length coded in the signal. Additionally, the energetic and predation cost of generating electric signals makes them costly handicaps.

To test the Reproductive Opportunity Model, I need a semelparous species that goes readily into homeostatic overload. The model species should have (i) limited opportunity for reproduction, (ii) energetically expensive communication signals, and (iii) it must be tractable to experimental manipulation and measurement. B. gauderio is also the perfect model to test the extreme predictions of the Reproductive Opportunity Model. Their short reproductive lifespan and high energy signal expense marks males of this species as candidates for routine homeostatic overload. Their EODs are also advantageous to study since the EOD's waveform reflects the hormonal profile of the signaler (Stoddard et al., 2006; Gavassa et al., 2011). The Stoddard lab has developed the equipment needed to continuously record calibrated electric signals from freely behaving fish for as many days as necessary (Stoddard et al., 2003). Moreover, B. gauderio are easily bred in the lab, but they can also be reliably found in the field in Uruguay.

Does signal plasticity compromise the reliability of the information conveyed by the signal, or instead, does it allow the signal to convey more current information? Do plastic signals allow individuals to better navigate through the trade-offs of costly signaling, mate attraction, and intrasexual competition while retaining sufficient information to stay relevant to receivers? 
I present the data from my dissertation in five chapters (Chapters 2-6). In Chapter 2, I investigate the effect of signal plasticity on the reliability of the information conveyed by the signal. I tested whether the ability to predict the size of the signaler from the amplitude of its EOD changes as the signaler enhances its EOD amplitude in response to competition. I compared changes in signal reliability in a natural population, estimating natural variation in population density. I tested the causality of the relationships found in the field with laboratory manipulations of population density. The combined field and lab approach provides a powerful test for the disposable handicap hypothesis. I found that as competition increases and promotes the enhancement of EOD amplitude, the reliability by which body size can be predicted from EOD amplitude increases. Therefore, I show that signal plasticity improves the information conveyed by the signal rather than compromising it.

In Chapter 3, I studied whether the plasticity of the EOD would help the signal convey current state of the signaler. The duration of the EOD's second phase is regulated by androgens, and androgens regulate multiple reproductive traits including gonad maturation and aggression. Thus, I expected the duration of the EOD's second phase to be a good indicator of androgen levels and other androgen-mediated traits. I tested whether the duration of the EOD's second phase correlated with androgen levels, gonad size, and sexual maturation. I recorded the EOD's and sampled blood from 155 individuals in the field. For each I measured androgen levels, gonad size, and sexual maturation. I found that the duration of the EOD's second phase indicates circulating androgen levels, but more importantly, androgens link the duration of the EOD's second 
phase to other androgen-mediated traits. As a result, the duration of the EOD's second phase is an honest indicator of reproductive condition in males, and estradiol levels in females.

In Chapter 4, I tested whether females would modify their electric signals in response to changes in the social environment, as males do. I compared the signal parameters of females recorded in the field to the characteristics of the natural population where the females inhabited. Later in the lab, I tested the direct effects of population structure on female signaling behavior by manipulating the social environment and recording the EODs from the females. I found that females increase the duration of the EOD's second phase when the ratio of adult males to adult females becomes femalebiased. Females also increase the amplitude of the EOD in response to an increased population density.

In Chapter 5, I investigated whether B. gauderio, a semelparous species with a relatively long breeding season, would use signal plasticity to reduce allostatic load and compromise signaling effort when food availability was low. I increased allostatic load by reducing food availability while providing incentive for costly signaling using a staged challenge from a conspecific. In order to assess the degree of allostatic load, I measured cortisol levels. I also measured androgen levels to determine whether androgens increased or decreased in response to allostatic load. I found that when food was limited, males further increased their investment on reproduction, as indicated by an increase in signaling parameters and in circulating androgen levels. The combination of the 
hormonal profile and behavioral response allowed me to infer a possible mechanism for B. gauderio to deal with stress.

In Chapter 6, I used a sender-based approach to test what signal parameters are important in a specific social context. Combining what I learned in Chapters 2 and 3 about what information is conveyed by each signal parameter and the context in which those parameters are enhanced, I aimed to elucidate the social function of EOD amplitude and duration. I found that males enhanced EOD amplitude in response to males but not to female social stimulus, while males elongated the duration of the EOD's second phase in response to both, male and female social stimuli. I suggest that males cue into information about body size coded by EOD amplitude and aggressiveness coded by pulse duration, while females may be primarily concerned about information on reproductive condition coded by pulse duration.

\section{References}

Adams, E. S., Mesterton-Gibbons, M., 1995. The cost of threat displays and the stability of deceptive communication. J. Theor. Biol. 175, 405-421.

Albert, J. S., Crampton, W. G. R., 2005. Diversity and phylogeny of Neotropical electric fishes (Gymnotiformes). Springer.

Allee, S. J., Markham, M. R., Stoddard, P. K., 2009. Androgens enhance plasticity of an electric communication signal in female knifefish, Brachyhypopomus pinnicaudatus. Horm. Behav. 56, 264-273.

Andersson, M., 1994. Sexual Selection. Princeton University Press, New Jersey.

Barry, T. P., Unwin, M. J., Malison, J. A., Quinn, T. P., 2001. Free and total cortisol levels in semelparous and iteroparous chinook salmon. J. Fish Biol. 59, 16731676.

Bass, A. H., Hopkins, C. D., 1983. Hormonal control of sexual differentiation: changes in electric organ discharge waveform. Science. 220, 971-4. 
Bass, A. H., Zakon, H. H., 2005. Sonic and electric fish: At the crossroads of neuroethology and behavioral neuroendocrinology. Horm. Behav. 48, 360-372.

Bee, M. A., Perrill, S. A., 1996. Responses to conspecific advertisement calls in the green frog (Rana clamitans) and their role in male-male communication. Behaviour. 133, 283-301.

Boonstra, R., 2005. Equipped for life: The adaptive role of the stress axis in male mammals. J. Mammal. 86, 236-247.

Boonstra, R., McColl, C. J., 2000. Contrasting stress response of male arctic ground squirrels and red squirrels. J. Exp. Zool. 286, 390-404.

Boonstra, R., McColl, C. J., Karels, T. J., 2001. Reproduction at all costs: The adaptive stress response of male Arctic ground squirrels. Ecology. 82, 1930-1946.

Bradley, A. J., 1987. Stress and mortality in the Red-tailed Phascogale, Phascogale calura (Marsupialia: Dasyuridae). Gen. Comp. Endocrinol. 67, 85-100.

Bradley, A. J., 1997. Reproduction and life history in the red-tailed phascogale, Phascogale calura (Marsupialia: Dasyuridae): the adaptive-stress senescence hypothesis. J. Zool. 241, 739-755.

Bradley, A. J., McDonald, I. R., Lee, A. K., 1980. Stress and mortality in a small marsupial (Antechinus stuartii, Macleay). Gen. Comp. Endocrinol. 40, 188-200.

Breuner, C. W., Patterson, S. H., Hahn, T. P., 2008. In search of relationships between the acute adrenocortical response and fitness. Gen. Comp. Endocrinol. 157, 288295.

Burmeister, S., Wilczynski, W., Ryan, M. J., 1999. Temporal call changes and prior experience affect graded signalling in the cricket frog. Anim. Behav. 57, 611-618.

Burmeister, S. S., Ophir, A. G., Ryan, M. J., Wilczynski, W., 2002. Information transfer during cricket frog contests. Anim. Behav. 64, 715-725.

Candolin, U., 1999. The relationship between signal quality and physical condition: is sexual signalling honest in the three-spined stickleback? Anim. Behav. 58, 12611267.

Candolin, U., 2000a. Changes in expression and honesty of sexual signalling over the reproductive lifetime of sticklebacks. Proc. R. Soc. Lond. B. 267, 2425-2430.

Candolin, U., 2000b. Increased signalling effort when survival prospects decrease: malemale competition ensures honesty. Anim. Behav. 60, 417-422.

Candolin, U., 2000c. Male-male competition ensures honest signaling of male parental ability in the three-spined stickleback (Gasterosteus aculeatus). Behav. Ecol. Sociobiol. 49, 57-61. 
Carlson, B. A., Hopkins, C. D., Thomas, P., 2000. Androgen correlates of socially induced changes in the electric organ discharge waveform of a mormyrid fish. Horm. Behav. 38, 177-186.

Carruth, L. L., Dores, R. M., Maldonado, T. A., Norris, D. O., Ruth, T., Jones, R. E., 2000. Elevation of plasma cortisol during the spawning migration of landlocked kokanee salmon (Oncorhynchus nerka kennerlyi). Comp. Biochem. Phys. C. 127, $123-131$.

Charlton, B., Ellis, W., McKinnon, A., Cowin, G., Brumm, J., Nilsson, K., Fitch, W., Cues to body size in the formant spacing of male koala (Phascolarctos cinereus) bellows: honesty in an exaggerated trait. J. Exp. Biol. 214, 3414-3422.

Charnov, E. L., Schaffer, W. M., 1973. Life-history consequences of Natural Selection: Cole's result revisited. Am. Nat. 107, 791-793.

Crampton, W. G. R., 1996. Gymnotiform fish: An important component of Amazonian floodplain fish communities. Journal of Fish Biology. 48, 298-301.

Creel, S., Creel, N. M., Monfort, S. L., 1996. Social stress and dominance. Nature. 379, 212-212.

Curtis, C. C., Stoddard, P. K., 2003. Mate preference in female electric fish, Brachyhypopomus pinnicaudatus. Anim. Behav. 66, 329-336.

Dawkins, R., Krebs, J. R., 1978, Animal signals: information or manipulation. In: J. R. Krebs, N. B. Davies, (Eds.), Behavioural Ecology: An Evolutionary Approach. Blackwell, Oxford, pp. 282-309.

Dunlap, K. D., 2002. Hormonal and body size correlates of electrocommunication behavior during dyadic interactions in a weakly electric fish, Apteronotus leptorhynchus. Horm. Behav. 41, 187-194.

Endler, J. A., 1992. Signals, signal conditions, and the direction of evolution. Am. Nat. 139, S125-S153.

Fitch, W. T., 1999. Acoustic exaggeration of size in birds via tracheal elongation: comparative and theoretical analyses. J. Zool. 248, 31-48.

Fitch, W. T., Hauser, M. D., 2002, Unpacking "honesty": vertebrate vocal production and the evolution of acoustic signals. In: A. Megela Simmons, A. N. Popper, (Eds.), Acoustic Communication. Springer-Verlag, New York, NY, USA, pp. 65-137.

Franchina, C. R., Salazar, V. L., Volmar, C. H., Stoddard, P. K., 2001. Plasticity of the electric organ discharge waveform of male Brachyhypopomus pinnicaudatus II. Social effects. J. Comp. Physiol. A. 187, 45-52. 
Franchina, C. R., Stoddard, P. K., 1998. Plasticity of the electric organ discharge waveform of the electric fish Brachyhypopomus pinnicaudatus. I. Quantification of day-night changes. J. Comp. Physiol. A. 183, 759 - 768.

Gautier, P., Barroca, M., Bertrand, S., Eraud, C., Gaillard, M., Hamman, M., Montreuil, S., Sorci, G., Faivre, B., 2008. The presence of females modulates the expression of carotenoid-based sexual signal. Behav. Ecol. Sociobiol. 62, 1159-1166.

Gavassa, S., Silva, A. C., Stoddard, P. K., 2011. Tight hormonal phenotypic integration ensures honesty of the electric signal of male and female Brachyhypopomus gauderio. Horm. Behav. 60, 420-426.

Goldina, A., Gavassa, S., Stoddard, P. K., 2011. Testosterone and 11-ketotestosteone have different regulatory effects on electric communication signals of male Brachyhypopomus gauderio. Horm. Behav. 60, 139-147.

Grafen, A., 1990. Biological signals as handicaps. J. Theor. Biol. 144, 517-546.

Grafen, A., Johnstone, R. A., 1993. Why we need ESS signaling theory. Philos. Trans. R. Soc. Lond. B. 340, 245-250.

Hagedorn, M., 1995. The electric fish Hypopomus occidentalis can rapidly modulate the amplitude and duration of its electric organ discharges. Anim. Behav. 49, 1409 1413.

Hagedorn, M., Carr, C., 1985. Single electrocytes produce a sexually dimorphic signal in South American electric fish, Hypopomus occidentalis (Gymnotiformes, Hypopomidae). J. Comp. Physiol. A. 156, 511-523.

Hopkins, C. D., 1981. The neuroethology of electric communication. Trends in Neurosciences. 4, 4-6.

Hopkins, C. D., 1999. Design features for electric communication. J. Exp. Biol. 202, 1217-1228.

Hopkins, C. D., Comfort, N. C., Bastian, J., Bass, A. H., 1990. Functional analysis of sexual dimorphism in an electric fish, Hypopomus pinnicaudatus, order Gymnotiformes. Brain Behav. Evolut. 35, 350-67.

Howard, R. D., Young, J. R., 1998. Individual variation in male vocal traits and female mating preferences in Bufo americanus. Anim. Behav. 55, 1165-1179.

Johnstone, R. A., 1997. The evolution of animal signals. Blackwell, Oxford.

Kim, T. W., Sakamotom, K., Henmi, Y., Choe, J. C., 2008. To court or not to court: reproductive decisions by male fiddler crabs in response to fluctuating food availability. Behav. Ecol. Sociobiol. 62, 1139-1147. 
Kokko, H., 1997. Evolutionarily stable strategies of age-dependent sexual advertisement. Behav. Ecol. Sociobiol. 41, 99-107.

Lindsay, W. R., Webster, M. S., Varian, C. W., Schwabl, H., 2009. Plumage colour acquisition and behaviour are associated with androgens in a phenotypically plastic tropical bird. Anim. Behav. 77, 1525-1532.

Liu, H., Wu, M. M., Zakon, H. H., 2008. A novel Na+ channel splice form contributes to the regulation of an androgen-dependent social signal. J. Neurosci. 28, 91739182.

Lorenzo, D., Silva, A., Macadar, O., 2006, Electrocommunication in Gymnotiformes: jamming avoidance and social signal during courtship. In: B. G. Kapoor, S. P. Collin, P. Moller, B. G. Kapoor, (Eds.), Communication in Fishes. Science Publisher, Inc., Enfield, NH, USA.

Markham, M., Allee, S., Goldina, A., Stoddard, P., 2009a. Melanocortins regulate the electric waveforms of gymnotiform electric fish. Horm. Behav. 55, 306-313.

Markham, M. R., McAnelly, M. L., Stoddard, P. K., Zakon, H. H., 2009b. Circadian and social cues regulate ion channel trafficking. PLoS Biol. 7, 1-14.

Markham, M. R., Stoddard, P. K., 2005. Adrenocorticotropic hormone enhances the masculinity of an electric communication signal by modulating the waveform and timing of action potentials within individual cells. J. Neurosci. 25, 8746-8754.

Maynard Smith, J., Harper, D. G. C., 1995. Animal signals: models and terminology. J. Theor. Biol. 177, 305-311.

McDonald, I. R., Lee, A. K., Bradley, A. J., Than, K. A., 1981. Endocrine changes in dasyurid marsupials with differing mortality patterns. Gen. Comp. Endocrinol. 44, 292-301.

McEwen, B. S., 1998. Stress, adaptation, and disease: allostasis and allostatic load. Ann. N. Y. Acad. Sci. 840, 33-44.

McEwen, B. S., 2000. Allostasis and allostatic load: Implications for neuropsychopharmacology. Neuropsychopharmacol. 22, 108-124.

McEwen, B. S., 2002. Sex, stress and the hippocampus: allostasis, allostatic load and the aging process. Neurobiol. Aging. 23, 921-939.

McEwen, B. S., Wingfield, J. C., 2003. The concept of allostasis in biology and biomedicine. Horm. Behav. 43, 2-15.

McGlothlin, J. W., Jawor, J. M., Greives, T. J., Casto, J. M., Phillips, J. L., Ketterson, E. D., 2008. Hormones and honest signals: males with larger ornaments elevate testosterone more when challenged. J. Evolution. Biol. 21, 39-48. 
McGraw, K. J., Hill, G. E., Parker, R. S., 2005. The physiological costs of being colourful: nutritional control of carotenoid utilization in the American goldfinch, Carduelis tristis. Anim. Behav. 69, 653-660.

Miranda, M., Silva, A., Stoddard, P. K., 2008. Use of space is consistent with exploded lek polygyny in the gymnotiform electric fish Brachyhypopomus pinnicaudatus. Environ. Biol. Fish. 83, 379-389.

Moller, P., 1995. Electric Fish: History and Behavior. Chapman \& Hall, London, NY, USA.

Murphy, C. G., 1994. Chorus tenure of male barking treefrogs, Hyla gratiosa. Anim. Behav. 48, 763-777.

Nur, N., Hasson, O., 1984. Phenotypic plasticity and the handicap principle. J. Theor. Biol. 110, 275-297.

Oakwood, M., Bradley, A. J., Cockburn, A., 2001. Semelparity in a large marsupial. Proc. R. Soc. Lond. B. 268, 407-411.

Otte, D., 1974. Effects and functions in the evolution of signaling systems. Annu. Rev. Ecol. Syst. 5, 385-417.

Parker, T. H., Knapp, R., Rosenfield, J. A., 2002. Social mediation of sexually selected ornamentation and steroid hormone levels in male junglefowl. Anim. Behav. 64, 291-298.

Proulx, S. R., Day, T., Rowe, L., 2002. Older males signal more reliably. Proc. R. Soc. Lond. B. 269, 2291-2299.

Reby, D., McComb, K., 2003. Anatomical constraints generate honesty: acoustic cues to age and weight in the roars of red deer stags. Anim. Behav. 65, 519-530.

Rohwer, S., Ewald, P. W., 1981. The cost of dominance and advantage of subordination in a badge signaling system. Evolution. 35, 441-454.

Romero, L. M., Dickens, M. J., Cyr, N. E., 2009. The reactive scope model - A new model integrating homeostasis, allostasis, and stress. Horm. Behav. 55, 375-389.

Ryan, M. J., 1985. The Túngara Frog. The University of Chicago Press, Chicago, IL, USA.

Ryan, M. J., Tuttle, M. D., Rand, A. S., 1982. Bat predation and sexual advertisement in a Neotropical anuran. The American Naturalist. 119, 136-139.

Salazar, V. K., The effect of male-male competition and its underlying regulatory mechanisms on the electric signal of the gymnotiform fish Brachyhypopomus gauderio., Biological Sciences. Florida International University, Miami, 2009, pp. 164. 
Salazar, V. L., Stoddard, P. K., 2008. Sex differences in energetic costs explain sexual dimorphism in the circadian rhythm modulation of the electrocommunication signal of the gymnotiform fish Brachyhypopomus pinnicaudatus. J. Exp. Biol. 211, 1012-1020.

Salazar, V. L., Stoddard, P. K., 2009. Social competition affect electric signal plasticity and steroid hormone levels in the gymnotiform fish Brachyhypopomus gauderio. Horm. Behav. 56, 399-409.

Sapolsky, R. M., Stress hormones: Good and bad. Neurobiology of disease. Academic Press Inc, 2000, pp. 540-542.

Schmidt, A. L., Taggart, D. A., Holz, P., Temple-Smith, P. D., Bradley, A. J., 2006. Plasma steroids and steroid-binding capacity in male semelparous dasyurid marsupials (Phascogale tapoatafa) that survive beyond the breeding season in captivity. Gen. Comp. Endocrinol. 149, 236-243.

Searcy, W. A., Nowicki, S., 2005. The Evolution of Animal Communication: Reliability and Deception in Signaling Systems. Princeton University Press, Princeton, NJ, USA.

Silva, A., Perrone, R., Macadar, O., 2007. Environmental, seasonal, and social modulations of basal activity in a weakly electric fish. Physiol. Behav. 90, 525536.

Silva, A., Quintana, L., Ardanaz, J. L., Macadar, O., 2002. Environmental and hormonal influences upon EOD waveform in gymnotiform pulse fish. J. Physiology-Paris. 96, 473-484.

Silva, A., Quintana, L., Galeano, M., Errandonea, P., 2003. Biogeography and breeding in gymnotiformes from Uruguay. Environ. Biol. Fish. 66, 329-338.

Silva, A., Zubizarreta, L., Costa, G., Interspecific differences in agonistic behavior and its serotonergic modulation. International Congress of Neuroethology, Salamanca, Spain, 2010.

Sterling, P., Eyer, J., 1988, Allostasis: a new paradigm to explain arousal pathology. In: S. Fisher, J. Reason, (Eds.), Handbook of life stress, cognition and health. John Wiley \& Sons, Chichester, pp. 750.

Stoddard, P. K., 1999. Predation enhances complexity in the evolution of electric fish signals. Nature. 400, 254-256.

Stoddard, P. K., 2002a. Electric signals: Predation, sex, and environmental constraints. Adv. Stud. Behav. 31, 201-242.

Stoddard, P. K., 2002b. The evolutionary origins of electric signal complexity. Journal of Physiology-Paris. 96, 485-491. 
Stoddard, P. K., 2006, Plasticity of the electric organ discharge waveform: context, mechanisms, and implications for electrocommunication. In: F. Ladich, S. P. Collin, P. Moller, B. G. Kapoor, (Eds.), Communication in Fishes. Science Publisher, Inc., Enfield, NH, USA, pp. 623-646.

Stoddard, P. K., Markham, M. R., 2008. Signal cloaking in electric fish. Bioscience. 58, 415-425.

Stoddard, P. K., Markham, M. R., Salazar, V. L., 2003. Serotonin modulates the electric waveform of the gymnotiform electric fish Brachyhypopomus pinnicaudatus. J. Exp. Biol. 206, 1353-1362.

Stoddard, P. K., Markham, M. R., Salazar, V. L., Allee, S., 2007. Circadian rhythms in electric waveform structure and rate in the electric fish Brachyhypopomus pinnicaudatus. Physiol. Behav. 90, 11-20.

Stoddard, P. K., Zakon, H. H., Markham, M. R., McAnelly, L., 2006. Regulation and modulation of electric waveforms in gymnotiform electric fish. J. Comp. Physiol. A. $192,613-624$.

Tibbetts, E. A., Dale, J., 2004. A socially enforced signal of quality in a paper wasp. Nature. 432, 218-222.

Tomkins, J., Kotiaho, J., LeBas, N., 2005. Phenotypic plasticity in the developmental integration of morphological trade-offs and secondary sexual trait compensation. Proc. R. Soc. Lond. B. 272, 543-551.

Wagner, W. E., 1992. Deceptive or honest signalling of fighting ability? A test of alternative hypotheses for the function of changes in call dominant frequency by male cricket frogs. Anim. Behav. 44, 449-462.

West-Eberhard, M. J., 1989. Phenotypic plasticity and the origins of diversity. Annu. Rev. Ecol. Syst.

Wiley, R. H., 1994, Errors, exaggeration, and deception in animal communication. In: L. A. Real, (Ed.), Behavioral mechanisms in evolutionary ecology. University of Chicago Press, Chicago, pp. 157-189.

Williams, G. C., 1966. Natural Selection, the costs of reproduction, and a refinement of Lack's Principle. Am. Nat. 100, 687-690.

Wingfield, J. C., 2005. The concept of allostasis: Coping with a capricious environment. J. Mammal. 86, 248-254.

Wingfield, J. C., Sapolsky, R. M., 2003. Reproduction and resistance to stress: when and how. J. Neuroendocrinol. 15, 711-724. 
Woods, H., Hellgren, E., 2003. Seasonal changes in the physiology of male Virginia opossums (Didelphis virginiana): signs of the Dasyurid semelparity syndrome? Physiol. Biochem. Zool. 76, 406-417.

Zahavi, A., 1975. Mate selection: a selection for a handicap. J. Theor. Biol. 53, 205-214.

Zahavi, A., 1977. The cost of honesty: further remarks on the handicap principle. J. Theor. Biol. 67, 603-605.

Zakon, H. H., McAnelly, L., Smith, T. G., Dunlap, K., Lopreato, G., Oestreich, J., Few, W. P., 1999. Plasticity of the electric organ discharge: implications for the regulation of ionic currents. J. Exp. Biol. 202, 1409 - 1416.

Zakon, H. H., Mills, A. C., Ferrari, M. B., 1991. Androgen-dependent modulation of the electrosensory and electromotor systems of a weakly electric fish. Semin. Neurosci. 3, 449-457. 


\section{FIGURES \& TABLES}

\begin{tabular}{lccccl}
\hline Species & $\begin{array}{c}\text { Breeding } \\
\text { season }\end{array}$ & $\begin{array}{c}\text { Breeding } \\
\text { days }\end{array}$ & $\begin{array}{c}\text { Breeding } \\
\text { intensity }\end{array}$ & $\begin{array}{c}\text { Cortisol } \\
\mathrm{ng} / \mathrm{ml}\end{array}$ & Reference \\
\hline Didelphis virginiana & $\begin{array}{c}\text { Jan-Feb \& } \\
\text { Apr-May }\end{array}$ & 120 & 3.0 & 2.6 & (Woods and Hellgren, 2003) \\
Dasyurus hallucatus & Apr-Jun & 120 & 3.0 & 3 & (Oakwood et al., 2001) \\
Antechinus stuartii & $2-3$ weeks & 18 & 20.0 & 65 & (Bradley et al., 1980) \\
Antechinus flavipes & $<2$ weeks & 14 & 25.7 & 39.85 & (McDonald et al., 1981) \\
Antechinus swainsonii & $<3$ weeks & 21 & 17.1 & 13.76 & (McDonald et al., 1981) \\
Phascogale tapoatafa & 4 weeks & 30 & 12.0 & 10 & (Schmidt et al., 2006) \\
Phascogale calura & 3 weeks & 21 & 17.1 & 30.78 & (Bradley, 1987) \\
\hline
\end{tabular}

Table 1.1. List of marsupial species used to test the reproductive opportunity model (see Fig. 3). For each species I present their respective duration of the mating season and cortisol levels for males during the mating season. Breeding intensity $=$ lifespan $/$ breeding days. Males of these species listed live up to one year. 


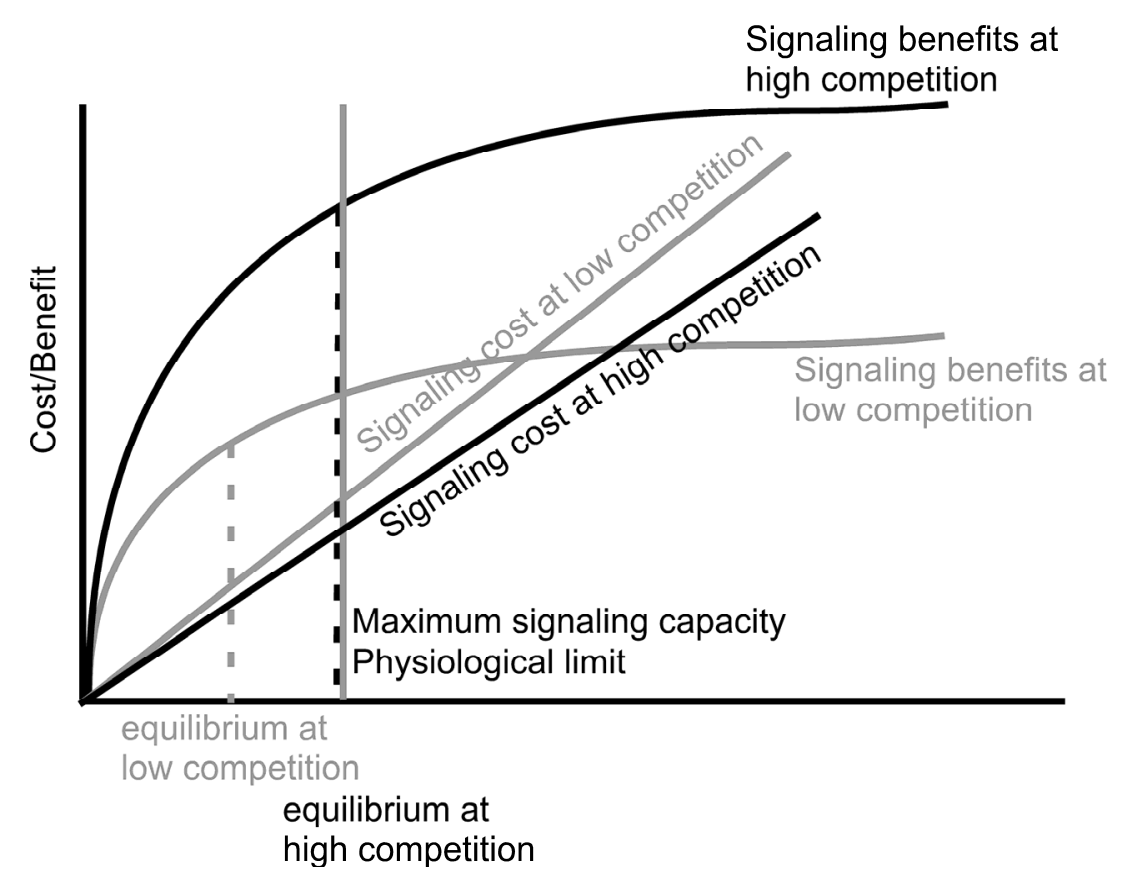

Figure 1.1. Graphical model of signaling equilibrium. Signaling benefits increase with signal intensity, while cost of signaling increase as well. Benefit function change with competition while cost function may either remain unchanged or decrease*. Equilibrium lies where the distance between the benefit and cost function is the highest (Adapted from Johnstone, 1997). * The cost function can decrease with competition, e. g. high population density dilutes predation risk. 


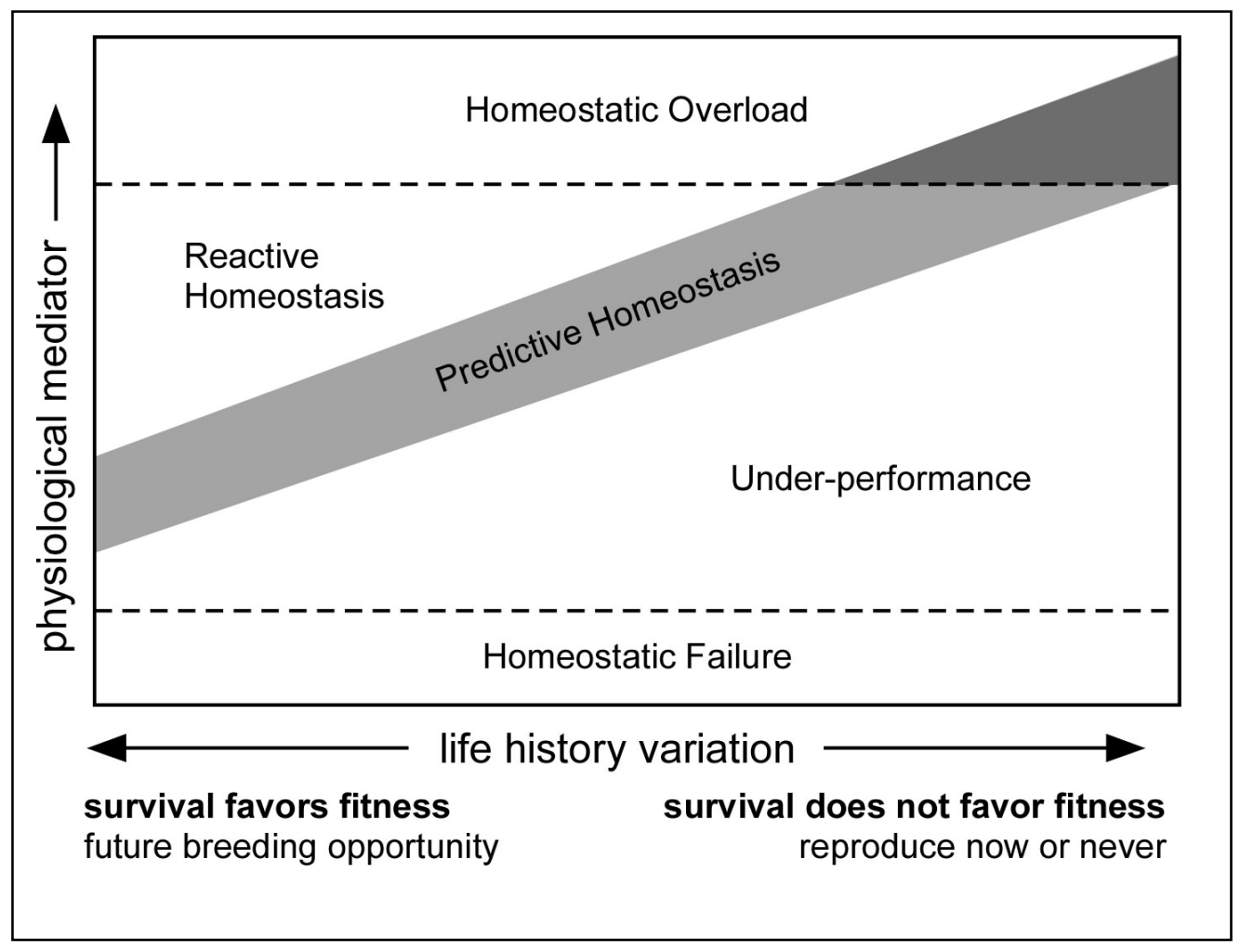

Figure 1.2. The Reproductive Opportunity Model predicts the homeostatic state of an individual based on its remaining opportunities for reproduction (Stoddard \& Gavassa, unpublished). 


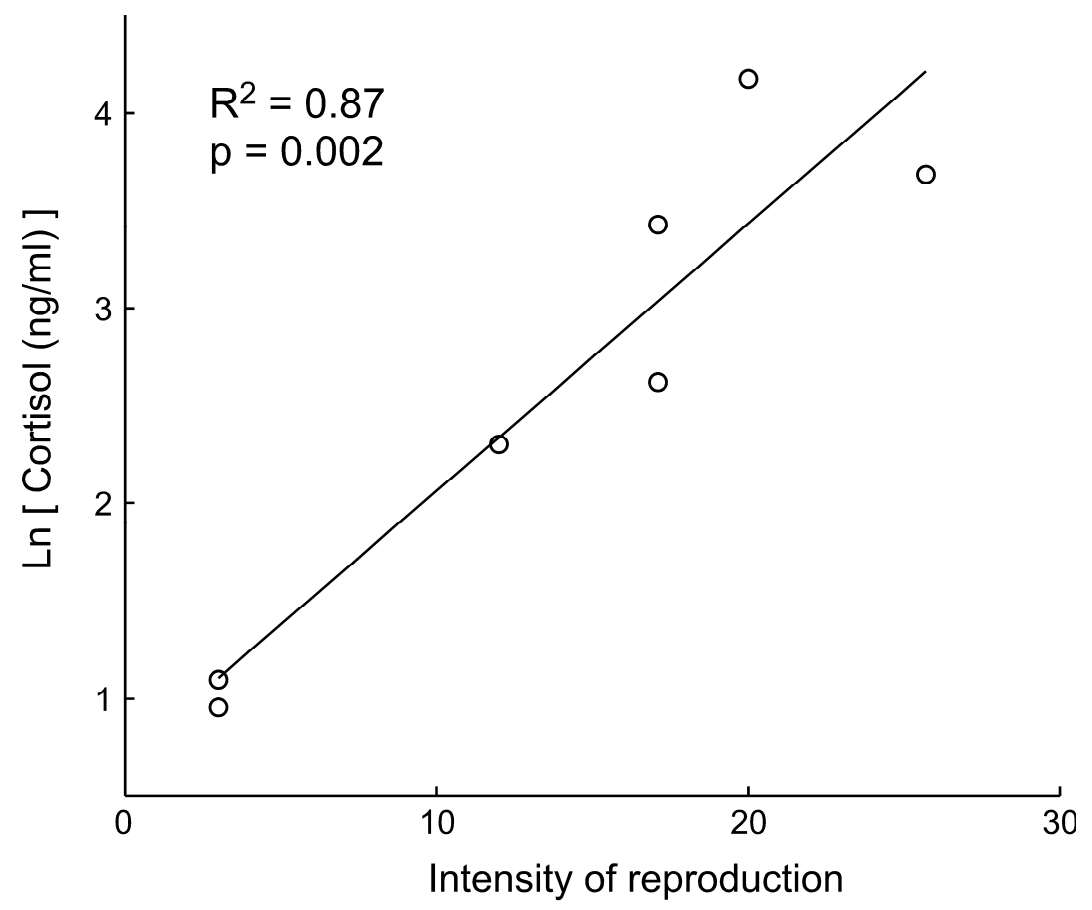

Figure 1.3. Natural logarithm of cortisol levels in males during the breeding season vs. intensity of reproduction. Marsupial males from species with short breeding seasons show the greatest levels of circulating cortisol during the breeding season. See Table 1 for species list. 


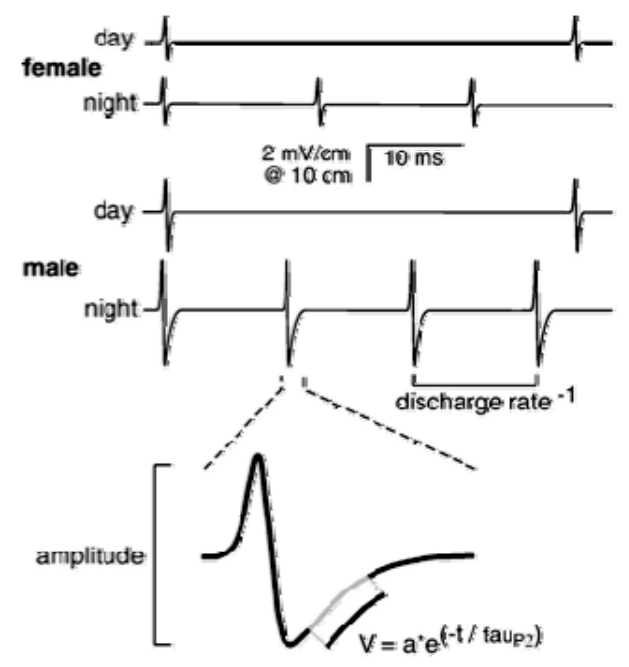

Figure 1.4. Sexually dimorphic electric organ discharge. Males and females increase amplitude and discharge rate at night. Duration of the second phase is computed as the repolarization time constant of the 2nd phase of the electric organ discharge. (Figure from: Stoddard et al., 2007). 


\section{CHAPTER 2}

SIGNAL MODULATION AS A MECHANISM FOR HANDICAP DISPOSAL

As published in Animal Behaviour

April 2012

Volume 83, Pages 935-944

Copyright (C) Elsevier 


\begin{abstract}
Signal honesty may be compromised when heightened competition provides incentive for signal exaggeration. Some degree of honesty might be maintained by intrinsic handicap costs on signalling or through imposition of extrinsic costs, such as social punishment of low-quality cheaters. Thus, theory predicts a delicate balance between signal enhancement and signal reliability that varies with degree of social competition, handicap cost and social cost. We investigated whether male sexual signals of the electric fish Brachyhypopomus gauderio become less reliable predictors of body length when competition provides incentives for males to boost electric signal amplitude. As expected, social competition under natural field conditions and in controlled laboratory experiments drove males to enhance their signals. However, signal enhancement improved the reliability of the information conveyed by the signal, as revealed in the tightening of the relationship between signal amplitude and body length. Signal augmentation in male $B$. gauderio was independent of body length, and thus appeared not to be curtailed through punishment of low-quality (small) individuals. Rather, all individuals boosted their signals under high competition, but those whose signals were farthest from the predicted value under low competition boosted signal amplitude the most. By elimination, intrinsic handicap cost of signal production, rather than extrinsic social cost, appears to be the basis for the unexpected reinforcement of electric signal honesty under social competition. Signal modulation may provide its greatest advantage to the signaller as a mechanism for handicap disposal under low competition rather than as a mechanism for exaggeration of quality under high competition.
\end{abstract}




\section{Introduction}

Dynamic signals may be reliable indicators of current quality since they can respond rapidly to changes in the individual's condition (Hill et al. 1999; Torres \& Velando 2003). However, the lability of dynamic signals may allow signallers to transiently escape signalling constraints and temporarily exaggerate their quality and thereby decrease signal reliability. Signal exaggeration can benefit a signaller when there is high reward incentive for increased signalling (Andersson 1994; Searcy \& Nowicki 2005). Although signalling systems can survive a small degree of unreliability without completely impairing the stability of the communication system (Johnstone \& Grafen 1993; Kokko 1997), a high prevalence of cheating in the population would render signals meaningless for receivers and result in receivers ignoring the signals (Dawkins \& Krebs 1978; Johnstone \& Grafen 1993). Thus, for signals to remain evolutionarily stable, dishonesty has to be contained.

According to the handicap principle, signal honesty is maintained when the advertising signal meets the following three conditions: (1) the expression of the signal depends on a sexually selected phenotypic trait of the signaller, (2) signals are expensive to produce or maintain and (3) the costs of signalling rise faster than its benefits (Grafen 1990; Zahavi 1975, 1977). A signal that satisfies the first condition is referred to as an 'index' if it is physically associated with the sexually selected trait of the signaller (Maynard Smith \& Harper 1995). Signalling cost may include the physiological cost of producing or maintaining the signal, the cost of predation risk that comes with increased conspicuousness due to signalling, and/or social cost from increased confrontation with conspecific males. 
Signallers are thought to benefit by producing costly signals when signalling incentive is high, such as during intensified competition, high resource value, or when future reproductive opportunities are reduced. Empirical studies show that competition often induces males to increase signalling effort, producing more conspicuous and costly signals (Kodric-Brown \& Brown 1984; Ryan 1985; Endler 1995; Franchina et al. 2001; Salazar \& Stoddard 2009). Likewise, males typically signal more in the presence of females (Akre \& Ryan 2011), and particularly in the presence of good-quality females (Wong \& Svensson 2009). Males also tend to signal more intensively when they are close to their last reproductive opportunity (Candolin 2000a; Proulx et al. 2002; Hall et al. 2009). Older males may benefit by reallocating energy from self-maintenance and survival to signalling if their survival probability has been reduced by age or time of season (Kokko 1997; Proulx et al. 2002; Lindstrom et al. 2009). Still, theory is unclear what effect signal enhancement should have on the reliability of the signal (Johnstone et al. 2009). Theoretical models predict that the marginal cost of signal augmentation should ultimately determine how signal enhancement affects honesty (Johnstone et al. 2009). (1) Low augmentation costs favour signal enhancement that reduces signal reliability (Zahavi 1975; Grafen 1990; Wagner 1992). (2) If signal enhancement is prevented by social costs, such as punishment of low-quality cheaters by social dominants, signal reliability should increase under social competition as low-quality signallers contain their signal output more than high-quality signallers (Rohwer \& Ewald 1981; Candolin 2000b). (3) Likewise, where signal enhancement disproportionally increases signal production costs, signal enhancement could reinforce the handicap effect and result in increased signal honesty (Wagner 1992; Johnstone et al. 2009). 
An imbalance between incentive and signalling costs can decrease signal reliability. When the benefits of signalling outweigh the costs, signal reliability is decreased by 'cheating up' wherein low-quality individuals signal at a level higher than expected for their quality (Wagner 1989a; Bee et al. 2000; Candolin 2000c). However, when the costs of signalling outweigh its benefits, signal reliability can also be reduced by 'cheating down' wherein high-quality individuals reduce their signalling output and reallocate that energy to self-maintenance as an investment in future reproduction (Lindstrom et al. 2009) or predation avoidance (Endler 1987). An expensive signal that can be attenuated to reduce costs under low competition is effectively a 'disposable handicap'.

From this disposable handicap hypothesis we can derive three testable predictions. (1) Signalling output should track incentive, such as social competition. (2) Signal reliability should increase under social competition as individuals increase their handicap magnitude and approach their physical limits. (3) Individuals that have lowered their signals the farthest below the level predicted for their quality have disposed of most of their handicap but will have the most to catch-up to when competition resumes. Thus, low-signalling individuals, rather than low-quality individuals, should enhance their signals the most when social competition increases.

We tested these predictions in Brachyhypopomus gauderio (Giora \& Malabarba 2009), a South American electric fish that produces a biphasic electric organ discharge (EOD). As with all gymnotiforms, the signal functions for active electrolocation and communication during their nocturnal active phase (Moller 1995). EODs of male $B$. gauderio and its sister species B. pinnicaudatus are greater in amplitude and second phase duration than those of females (Hopkins et al. 1990; Franchina \& Stoddard 1998). 
Moreover, the EOD of B. gauderio has the potential to serve as an indicator of body length since the amplitude of the signal physically depends on the length of the electric organ, which runs the length of the fish's body (Hopkins et al. 1990; Hopkins 1999; Curtis \& Stoddard 2003). Body length information is relevant for mate choice and malemale interactions, since longer males are more attractive to females (Curtis \& Stoddard 2003) and more likely to win agonistic encounters (Salazar 2009; A. Silva, L. Zubizarreta \& G. Costa, unpublished data). Additionally, electric signals of males are costly handicaps; they are energetically expensive, consuming an average of $22 \%$ of the daily energetic budget (compared to 3\% for females), and their energetic costs rise with the amplitude of the EOD (Salazar \& Stoddard 2008). However, the EOD of B. gauderio is highly plastic, varying in magnitude along multiple timescales (Stoddard et al. 2006). EOD amplitude and duration display short-term changes, both increasing during social interaction (Franchina et al. 2001; Silva et al. 2007) and in anticipation of night, when fish become active (Franchina \& Stoddard 1998; Stoddard et al. 2007). These short-term signal increases are mediated by melanocortin peptide hormones such as ACTH and $\alpha$ MSH (Markham \& Stoddard 2005; Stoddard \& Markham 2008; Markham et al. 2009a). Social interaction also induces long-term changes across several days (Franchina et al. 2001), and signals vary seasonally with the transition into reproductive condition (Silva et al. 2002). These long-term signal enhancements are mediated by androgens (Silva et al. 2002; Allee et al. 2009; Gavassa et al. 2011; Goldina et al. 2011).

Here we test whether signal enhancement affects signal reliability, and if so, in which direction. We studied the reliability of EOD amplitude as an indicator of body length, the main determinant of male-male competition and female choice. Given the 
cost of the electric signal of $B$. gauderio and the intrinsic relation between body length and EOD amplitude, signal modulation could be used to reduce signalling cost when signalling incentives are low (i.e. 'cheating down'). Conversely, the cost of signal enhancement may not be high enough to prevent dishonest exaggeration, in which case some individuals should be able to enhance their signal beyond their quality (i.e. 'cheating up').

\section{Materials and Methods}

\section{$\underline{\text { Subjects }}$}

We studied B. gauderio (Giora \& Malabarba 2009), sister species of $B$. pinnicaudatus (Hopkins et al. 1990), native to marshes and slow waters of Argentina, Uruguay and southern Brazil. Males have longer and broader caudal filaments than females and produce EODs of greater amplitude and longer duration than females. Brachyhypopomus gauderio is a short-lived species; adults disappear from natural populations after breeding during the austral summer (Silva et al. 2003), and males disappear faster than females (Miranda et al. 2008). Males maintain territories and females move freely between male territories, consistent with exploded lek polygyny (Miranda et al. 2008). We studied B. gauderio from a native population in Uruguay, and from our 18th generation captive-reared colony in Miami, FL, U.S.A. Collections and experimental procedures were performed under the guidelines and approval of the Comisión Honoraria de Experimentación Animal, Universidad de la República, Montevideo, Uruguay, and by the Institutional Animal Care and Use Committee of the Florida International University, Miami, FL (protocols 08-027 and 10-020). 


\section{Field Study}

Detailed field methods are described by Gavassa et al. (2011). In brief, we recorded the EODs of B. gauderio in situ from Laguna Lavalle $\left(32^{\circ} 01.259\right.$ 'S, $055^{\circ}$ 22.498'W), Department of Tacuarembó, Uruguay. We sampled during 15, 16, 27 and 28 October, 16-18 November, and 4, 12 and 13 December. Samples from consecutive days were pooled. All sampling took place during the breeding season reported for this species in this region of the Southern Hemisphere (Silva et al. 2003). All sampling occurred during the day (1100-1759 hours Uruguay Summer Time, UYST) on sites with similar depth and vegetation located along the shore of the lagoon. We located fish in their hiding places using an amplifier to convert their electric signals into sound. We recorded EODs of captured fish in a floating digitizer rig within 1 min of initial disturbance, before melanocortin-mediated EOD waveform modulation could take effect (Stoddard et al. 2006). We performed a census in each of the seasonal sampling sites to estimate population abundance. We estimated the abundance of $B$. gauderio by recording the time a skilled fishing team took to capture 30 fish, and defined population abundance in terms of fish captured per unit of effort (CPUE fish/h). This method has been very successful for estimating population abundance in this species since these fish are nocturnal and spend the day motionless in their hiding place where they are easily captured (Silva et al. 2003; Miranda et al. 2008).

We confirmed the sex of the fish by gonadal inspection after sacrifice by immersion in an anaesthetic solution (eugenol $8 \mathrm{mg} /$ litre). The body lengths of males collected ranged between 10.5 and $19.3 \mathrm{~cm}$, corresponding to the size of adult males (Franchina 1997; Silva et al. 2003). Caudal filament damage affects the EOD waveform 
(Hopkins et al. 1990); thus, only fish with intact caudal filaments were retained for EOD analysis. In each sampling event we captured 10 or more males, except for 12-13 December, when we only captured eight males, two of which had damage to their caudal filaments and could not be used for EOD analysis.

\section{Laboratory Experiment}

We tagged 18 females and 18 males with alphanumeric elastomer tags and randomly placed them in 450-litre pools outdoors in groups of 2, 6, 12 or 18 fish, keeping sex ratio at unity. The body length of males used in this experiment ranged between 15.5 and $24.0 \mathrm{~cm}$. At a given time we had six pools with two fish each, two pools with six fish each and one pool with 12 fish, this set-up was replicated three times and followed by two pools with 18 fish each. This design manipulated fish density while controlling for sex ratio. After 1 week of social treatment, we recorded EODs of all fish. In between social treatments, fish remained with an opposite-sex companion for 1 week. All fish except two (one male and one female that became sick) underwent all treatments; the two fish that became sick were replaced partway through the experiment. Treatments were temporally interspersed, except for the 18-fish pool, which was performed at the end because it required using most of the experimental individuals.

In B. gauderio, the effect of temperature on EOD amplitude, $Q_{10}$, is nonlinear (Silva et al. 2002), wherein EODs of sexually mature males partially resist effects of declining temperature (Silva et al. 1999). An early cold front arrived during the week of the 18 -fish/pool treatment and lowered the temperature of the holding pools $6{ }^{\circ} \mathrm{C}$ below the average for the other treatments. Brachyhypopomus gauderio remains socially active over wide temperature swings, and all fish in the 18-fish treatment experienced the same 
temperature, so we expected the intragroup signal dynamics to continue to reflect the pool density. Thus, although we could not directly compare the absolute values of EOD amplitude in the $18 \mathrm{fish} /$ pool treatment with the other treatment groups that experienced narrower temperature ranges, we did use these data in within-group amplitude analyses.

\section{EOD Recordings and Analysis}

In gymnotiforms, the repeatability of the recordings depends on the orientation of the fish and its relative distance to the recording electrodes (Hopkins 1986). Field EOD recordings took place inside the lagoon in a submerged plastic sheet cage $(100 \times 50 \times 50$ $\mathrm{cm}$ ) with recording electrodes located at either end, $100 \mathrm{~cm}$ apart. A mesh tube held the fish lengthwise, equidistant from the recording electrodes and $25 \mathrm{~cm}$ below the water surface. A ground electrode was located perpendicular to the fish. This EOD recording geometry was designed to permit high repeatability of amplitude measurements (Franchina \& Stoddard 1998). Water conductivity was $38-57 \mu \mathrm{S} / \mathrm{cm}$ and water temperature was $19-28{ }^{\circ} \mathrm{C}$ throughout the field study. The variability in environmental conditions and the underlying seasonal variability associated with field observations make laboratory tests crucial to confirm field observations. In the laboratory, we recorded the EODs inside a glass aquarium $(120 \times 40 \times 40 \mathrm{~cm})$, with water conductivity adjusted to $100 \pm 6 \mu \mathrm{S} / \mathrm{cm}$ and $25.8 \pm 1.3{ }^{\circ} \mathrm{C}$. Since we did not cross-calibrate the recording rigs, which differed in dimensions and water conductivity between field and laboratory, we could not directly compare absolute amplitude measurements of these populations.

The EODs were differentially amplified 100x (World Precision Instruments, Inc., Sarasota, FL, U.S.A., DAM-50, AC-coupled, low-pass filter corner $0.1 \mathrm{~Hz}$, high-pass filter corner $10 \mathrm{kHz}$ ). Signals were digitized by an RM1 mobile processor (Tucker Davis 
Technologies, Alachua, FL, U.S.A.), and stored and analysed on a portable computer using custom software developed in MatLab (Mathworks, Natick, MA, U.S.A.). We analysed the EODs using custom MatLab software to calculate median values of peak-topeak amplitude (mV) (Stoddard et al. 2003).

\section{$\underline{\text { Model Fitting and Statistics }}$}

Following Hughes (2000), we analysed the honesty of the signal as a remote indicator of body length by using signal residuals obtained from the regression of signal amplitude and body length. Based on the visual inspection of the scatter plots, we tested the fit of several regression models: linear, exponential, noninteger exponential and power function. We tested the validity of these regression models by checking whether their residuals were normally distributed, with a mean of zero, independent, uncorrelated and homeoscedastic. When we regressed EOD amplitude against body length using a simple linear regression model, the residuals were still correlated with body length $(r=$ 0.68, $P<0.0001$ ), indicating higher-order or nonlinear relationship (Fig. 1). The residual plot also showed heteroscedasticity of the error. Linear regression of $\ln (\mathrm{EOD}$ amplitude) against body length provided a strong fit while satisfying all the assumptions of linear regression; therefore, we used $\ln (\mathrm{EOD}$ amplitude) to determine reliability of EOD amplitude as an honest indicator of body length. Differences between group means were tested using ANOVA for field observations or repeated measures ANOVA for laboratory manipulations. When significant differences were detected $(P<0.05)$ by the ANOVA models, we used protected Tukey post hoc analysis to determine pairwise statistical differences at $\alpha=0.05$ level. All statistical analyses were performed using the MatLab Statistical Toolbox and SPSS v.18 (SPSS, Chicago, IL, U.S.A.). 


\section{Results}

\section{Signalling Incentive and Signalling Output}

The first prediction of the disposable handicap hypothesis is that males should modulate their signal output in proportion to social incentive, such as intensity of competition. In the field, the local population density in the breeding marshes changed from one sampling event to the next by as much as a factor of two (CPUE: 5, 8, 4.5, 10 and 5.7 fish/h). The highest mean signal amplitude in the field occurred at the highest population density (ANOVA: $F_{4,50}=6.39, P<0.001$; Fig. 2 a). Moreover, residual signal amplitude adjusted for body length correlated significantly with population density, while sex ratio, season and temperature showed no relation to signal amplitude (Spearman correlations: population density: $r_{\mathrm{s}}=0.55, N=55, P<0.001$; sex ratio: $r_{\mathrm{s}}=-0.08, N=55$, $P=0.54$; season: $r_{\mathrm{s}}=0.11, N=55, P=0.40$; temperature: $\left.r_{\mathrm{s}}=0.23, N=55, P=0.08\right)$. Our experimental laboratory study confirmed the effect of population density on signal amplitude: males increased signal amplitude in the high-density treatments $(6,12$ and 18 fish/pool) with respect to the low-density treatment (repeated measures ANOVA: $F_{1,17}=$ 71.64, $P<0.001$; Fig. 2b).

\section{Signalling Incentive and Signal Reliability}

The second prediction of the disposable handicap hypothesis is that signal reliability should increase with competition. In the field, we found a strong relationship between EOD amplitude and body length throughout the season, but the strength of the association varied with population density (Fig. 3 a-e). When field population density was the highest, the residuals from the regression between $\ln (\mathrm{EOD}$ amplitude) and body length had the lowest variance compared to any other sample (Bartlett test: $\chi^{2}{ }_{4}=10.95, P=$ 
0.027). In the laboratory, only the highest-density treatments had significant relationships between EOD amplitude and body length (Fig. 3k-n). As predicted from the field data, experimental manipulation of fish density in the laboratory determined the reliability of body length as a predictor of electric signal amplitude. Under both natural field conditions and experimental laboratory conditions, the relation between body length and signal amplitude became stronger with population density (field: $R^{2}=0.95, N=5, P=$ 0.004 , post hoc statistical power $=0.99$; laboratory: $R^{2}=0.98, N=4, P=0.006$, post hoc statistical power $=0.99$; Fig. 4 ). Thus, the signal became more reliable with increased competition.

We defined the accuracy of EOD amplitude as a predictor of body length by calculating the standard deviation of the residuals from the regression of body length and $\ln$ (EOD amplitude) as a percentage of body length (100 x body length residuals/body length), encompassing $68 \%$ of the population (Fig. $3 \mathrm{~s}$ ). In the field, the accuracy with which EOD amplitude predicted the size of males varied from 7.3\% at low density (Fig. $3 \mathrm{f}-\mathrm{i}$ ) to $2.4 \%$ at the highest density (Fig. $3 \mathrm{j}$ ). That is, at the highest density in the field, EOD amplitude could be used to predict the body length of $68 \%$ of males to within $2.4 \%$ of actual body length. The accuracy of EOD amplitude as a predictor of body length was lower in the laboratory, where EOD amplitude could predict body length with an accuracy of $9.1 \%$ at most (Fig. 3o-r).

\section{$\underline{\text { Handicap Disposal versus Social Cost }}$}

The third prediction of the disposable handicap hypothesis is that males whose signal amplitudes are farthest below those predicted for their body lengths under low competition should enhance their signals the most under high competition to reveal their 
full signalling capability. Comparison of males in the laboratory experiment that had experienced both low and high density ( 2 versus 12 fish/pool, random order) revealed that every male generated signals of higher amplitude under high social competition than under low social competition (paired $t$ test: $t_{15}=8.2$, one-tailed $P<0.001$ ). Likewise, males whose signal amplitudes were the farthest below the body length regression line under low competition ( 2 fish/pool) increased signal amplitude the most under high competition (12 fish/pool) $\left(R^{2}=0.45, N=16, P=0.004\right.$; Fig. 5a). Body length did not predict the amount of amplitude change $\left(R^{2}=0.02, N=16, P=0.63\right.$; Fig. $\left.5 \mathrm{~b}\right)$, inconsistent with the hypothesis that social punishment might suppress small males from enhancing their signals.

If social costs are constraining signal reliability, we would predict larger males to increase signal amplitude more than small males during high competition (Candolin 2000b). However, neither in the field nor in the laboratory did the slope or the intercept of the regressions between body length and $\ln (\mathrm{EOD}$ amplitude) differ significantly between population densities (ANCOVA: field: $F_{4,45}=1.82, P=0.141$; laboratory: $F_{3,63}$ $=0.36, P=0.78)$. Thus, we found no evidence that social costs constrained enhancement of EOD amplitude.

\section{Discussion}

We sought to determine whether the ability of gymnotiforms to augment their electric signals could allow individuals to exaggerate their quality, in the process, reducing the reliability of their signals as indicators of body size. Alternatively, given the high energetic expense of the EOD, males might modulate EOD amplitude for the 
opposite function, to reduce signal magnitude and dispose of handicap costs when the incentive for costly signalling is low. Under the disposable handicap model, high competitive incentive would pull signallers upward towards the physiological bound of their signalling capability where the signal serves as a reliable index of size or quality. In our studies, EOD amplitude was highest when population abundance was highest, showing that competition did provide incentive for enhanced signalling. Such enhanced signalling also increased reliability of the signal as an index of body length. Thus, signal enhancement no longer functioned to exaggerate body size; on the contrary, it resulted in a more reliable indication of body size. These findings support the predictions of the 'disposable handicap' hypothesis and contradict the hypothesis that signal enhancement degrades signal reliability.

Information Coded by the Amplitude of the EOD

We expected electric signals of male B. gauderio to code information about body length since body length is the main determinant both of female preference and of malemale contest outcome. In the laboratory, females select longer males, which typically produce EODs of greater amplitude and duration (Curtis \& Stoddard 2003). Moreover, in staged resident-intruder contests, the longest male always wins the encounter despite residential status or body condition (Salazar 2009; A. Silva, L. Zubizarreta \& G. Costa, unpublished data). From the tight relationship between body length and EOD amplitude, a receiver could use the amplitude of the signaller's EOD as an indicator of his resourceholding potential. Although signal reliability increased with competition in both the laboratory and the field, signal reliability was slightly greater in the field. Our laboratory 
fish are accustomed to high densities, possibly making it harder to simulate increased competition.

Although it has not been shown that gymnotiforms use electric signals to estimate body length of conspecifics, detailed laboratory studies indicate that electric fish have the sensory capability to disambiguate strong, distant signals, from weak, close signals. Electric fish can determine the distance of an active signal source from the curvature of the field lines. Gymnotus carapo and Brachyhypopomus diazi follow the curvature of field lines to locate electric sources (Davis \& Hopkins 1988; Shieh et al. 1996), but Gymnotus will cut across the field lines to attack a familiar signaller, showing it has analysed the field structure to separate signal intensity and source location (Scudamore \& McGregor 1993). Mormyrids have been shown to distinguish between a large distant object, and a close small object (von der Emde et al. 1998). From these studies we infer that signal amplitude is not made ambiguous by source distance, so receivers can most likely ascertain EOD amplitude independently of distance to obtain information about the signaller.

\section{$\underline{\text { EOD Modulation as a Mechanism for Handicap Disposal }}$}

Both field and laboratory results satisfy the first prediction of the disposable handicap hypothesis, that males should modulate their signals in proportion to signalling incentive, such as competition intensity. Prior laboratory studies had also found that EOD amplitude of male B. gauderio varied with social context (Franchina et al. 2001), increasing with the number and proportion of male fish in the pool (Salazar \& Stoddard 2009). 
The second prediction of the disposable handicap hypothesis is that signal reliability increases with competition. This prediction is key to the hypothesis since it distinguishes between signal enhancement for dishonest versus honest signalling. If signal modulation is used to exaggerate body length (cheating up), the signal should be the least reliable under high competition, whereas if signal modulation is used to economize under low competition (cheating down), the signal should be least reliable under low competition. Consistent with the disposable handicap hypothesis, the reliability of the signal was lowest when signal intensity and signalling incentives were low (cheating down), while signal reliability increased with competition intensity.

Johnstone et al. (2009) proposed that when the marginal cost of signal enhancement rises disproportionally with signal intensity, the quality of the information conveyed by the signal improves with signal enhancement. Accordingly in B. gauderio, the energetic cost of producing a male EOD rises in proportion to EOD amplitude (Salazar \& Stoddard 2008). Given the exponential relationship between EOD amplitude and body length (Fig. 1a), for a male to effectively exaggerate his size, he would have to boost his EOD amplitude exponentially and bear the exponential cost of doing so. Furthermore, Salazar \& Stoddard (2008) showed that males spend 10 times more of their total energy budget in signalling than females. Moreover, males (but not females) trade-off between energy allocated to signals versus other metabolic compartments (Stoddard \& Salazar 2011). Additionally, by extending the duration of the second phase of the EOD, males divert energy to the low-frequency spectrum, detected by ampullary electroreceptors of predators (Stoddard \& Markham 2008), making male electric signals more conspicuous than female signals, and increasing their predation risk (Hanika \& Kramer 1999, 2000; 
Stoddard 1999, 2002). The increased predation risk and energetic metabolic trade-off of male signals should provide great incentive for handicap disposal during low competition.

The third prediction of the disposable handicap hypothesis is that social competition drives signal catch-up. Under low competition, males whose EOD amplitudes are farthest below those predicted for their body length should enhance their signals the most under high competition. In other words, the farther a male's amplitude has strayed from his individual limit under low signalling incentive, the greater his catch-up under high signalling incentive. This prediction allows us to distinguish between signal modulation for energy savings and signal modulation to avoid social costs; the latter would be concentrated among smaller individuals that face punishment for appearing larger than they are. We found that the magnitude of male EOD enhancement under social competition depends on the male's prior signal magnitude and capacity for increase rather than on his quality (size). Under a social costs constraint, we also would have expected larger males to increase signal amplitude more than small males during high competition, resulting in a steeper relationship between body length and $\ln (\mathrm{EOD}$ amplitude) under high competition than under low competition (Candolin 2000b). But we found no evidence that social costs prevent small, low-quality males from increasing signal amplitude. The slopes of the regressions between body length and $\ln (\mathrm{EOD}$ amplitude) did not differ significantly between low and high competition, disfavouring the idea that signal enhancement is constrained by social costs. 


\section{Mechanisms Ensuring Honesty}

In addition to constraints from energetic expense and predation risk, signal exaggeration by male $B$. gauderio might be subject to allometric or biophysical constraints. For instance, electric signal amplitude might be limited either by the number of electrocytes that can be packed into a given length of fish, or by sodium channel retention levels in electrocyte membranes. Animals in which the degree of signal expression is physically linked to the sexually selected trait of the signaller presumably cannot exaggerate their signals (cheat up) and thus their signals should be intrinsically honest (Maynard Smith \& Harper 1995). In frogs, the spectral frequency of the male's call is allometrically related to body size. The fundamental frequency of the call is determined in part by the size of the laryngeal apparatus, which varies with body length, resulting in a negative correlation between body length and fundamental frequency (Ryan 1980, 1985; Gerhardt 1994). Similar to the effect of competition on signal reliability in $B$. gauderio electric fish, in the toad, Bufo americanus, a decrease in fundamental frequency of the call results in a tighter relationship between frequency and body size (Howard \& Young 1998). However, some allometric constrains can be circumvented through evolution of bypass mechanisms wherein signal enhancement reduces signal honesty. For instance, in most of the anuran species studied, exaggeration of male body size through the dynamic reduction of call frequency degrades reliability of the signal as an indicator of body size (Wagner 1989b, 1992; Bee \& Perrill 1996; Bee et al. 2000).

Selection on signallers to circumvent allometric relationships may hit additional and more restricting constraints. Although the fundamental frequency of acoustic signals is determined by the size of the larynx, resonance in the acoustic path from the larynx to the 
external environment reinforces particular spectral frequencies, producing formant peaks. The frequency of those formants depends on the characteristics of the vocal tract, primarily the distance from the larynx to the external environment (Fitch \& Hauser 2010). Animals could potentially exaggerate their size by positioning the larynx deeper in the body, closer to the chest, therefore elongating their trachea to lower the pitch (Fitch 1999). Howbeit, a new constraint arises, as the larynx cannot be lowered beyond the top of the sternum (Reby \& McComb 2003). As a result, the active lowering of the larynx reinforces the relationship between body size and formant frequency, enhancing the honesty of formant frequency as an indicator of body size (Reby \& McComb 2003).

In electric fish, the amplitude of the EOD physically depends on the length of the fish: longer fish can accommodate more electrogenic cells, electrocytes, along their bodies and therefore produce EODs of greater amplitude (Hopkins et al. 1990; Hopkins 1999; Curtis \& Stoddard 2003). Furthermore, the electric field of Brachyhypopomus resembles a dipole, and separation of the dipole is proportional to the fish's length (Stoddard et al. 1999). Thus, longer fish have longer electric organs with greater dipole separation resulting in an exponential relationship between body length and EOD amplitude. At least two potential mechanisms could allow an increase in EOD amplitude outside the intrinsic relationship with body length. First, gymnotiform electric fish can increase the amplitude of their signals by augmenting the number of voltage-gated sodium channels in their electrocyte membranes (Markham et al. 2009b). Second, male B. gauderio can increase the temporal offset of the opposing action potentials in the biphasic EOD, which increases the EOD amplitude up to 25\% (Markham \& Stoddard 2005; Markham et al. 2009a). The latter mechanism merely unmasks the signal and thus 
should not require additional metabolic energy, whereas the former increases energy consumption in proportion to the opening of added ion channels. These mechanisms possibly evolved to exaggerate the signaller's quality. Nevertheless, these mechanisms could have reached their own intrinsic limits: (1) maximum number of sodium channels per electrocyte membrane, (2) maximum temporal offset of the two phases of the EOD, (3) maximum energy diverted to EOD production before other bodily functions begin to fail and/or (4) increased predation risk. Therefore, further constraints on size-independent mechanisms for electric signal enhancement still maintain a strong one-to-one relationship between body length and EOD amplitude.

\section{Adaptive Significance of the 'Disposable Handicap' Hypothesis for Receivers}

Receivers will benefit from obtaining the most accurate information from the signal, however, the benefits of receiving reliable signals may be greater under high competition than under low competition. Wiley's (1994) 'adaptive gullibility hypothesis' predicts that, under low competition, it costs more to incorrectly reject an honest signal than to believe a dishonest one, while under high competition, it costs more to believe a dishonest signal than to reject an honest one. For example, under low competition, the cost of finding a new territory might be lower than the cost of failing to believe a signal from a high-quality individual that could inflict damage while defending a contested territory. Conversely, under high competition, when territories are scarce, the cost of an agonistic encounter may be lower than the cost of suckering for a dishonest signal and abandoning attempts to obtain a particular territory. So, if signals become unreliable under high competition, they become virtually worthless to receivers, and thus not worth producing, whereas under low competition, receivers benefit by being more trusting, 
allowing signallers to cheat down a bit without losing their option for honesty under high competition when the reliability of signals matters more.

\section{$\underline{\text { Conclusion }}$}

While signal modulation by B. gauderio may have arisen as a mechanism for signal exaggeration, in the present day when every male has this capacity, signal modulation appears to benefit the individual by allowing a reduction in signalling costs during low competition (cheating down) rather than by effective exaggeration of body length during high competition (cheating up). This conclusion is supported by (1) downward modulation of signal amplitude during low competition, (2) increased predictive value of the signal (greater honesty) with elevated signal amplitude during increased social competition, (3) ubiquitous increase in signal amplitude with social incentive, independent of signaller quality and (4) the dependence of the magnitude of amplitude increase under high social incentive on deviance from the predicted value measured under low social incentive.

Signal modulation mechanisms that may have initially evolved under the selective advantage of exaggerating the signaller's quality could be maintained in the signaller by the advantages of energy conservation under low competition (i.e. a disposable handicap). Under this scenario, competition should improve the reliability of disposable handicaps as indicators of quality. The enhancing effect of signal augmentation on signal reliability suggests an evolutionarily stable strategy where signals still increase, but no receivers are fooled, and no signaller can afford not to boost his signal. Thus, attention to signals by receivers should be reinforced, rather than degraded, by the signaller's capacity to enhance the signal, provided the signal costs rise with the signal output faster 
than the increasing benefits of enhanced signalling. Furthermore, handicap disposal (energetic savings) could have served as the originating driver of signal modulation just as easily as exaggeration of body size.

\section{Acknowledgments}

We thank R. Perrone, L. Zubizarreta, G. Batista, T. de los Campos, D. Colacce, I. Becerra, P. Pouso, A. Cabana and J. E. Campbell for assistance in the field. We thank Dr O. Macadar, Min. de Cultura y Educación, Uruguay, and Fac. de Ciencias, Universidad de la República, Montevideo, Uruguay, for field logistics. We thank J. Molina, A. Goldina, C. Curtis, P. Perez and J. Roach for assistance in the lab. We thank two anonymous referees for their helpful comments on the manuscript. This work was supported by Tinker Travel Grant (LACC at FIU), FIU Graduate School's Dissertation Evidence Acquisition Fellowship, Judith Evans Parker Scholarship, Animal Behavior Society Graduate Student Award to S.G., National Institutes of Health MBRS RISE R25 award GM061347 to C. H. Bigger, and National Science Foundation grant IOS 0956603 to P.K.S. This paper is contribution number 215 to the FIU Tropical Biology Program. 


\section{References}

Akre, K. L. \& Ryan, M. J. 2011. Female túngara frogs elicit more complex mating signals from males. Behavioral Ecology, 22, 846-853.

Allee, S. J., Markham, M. R. \& Stoddard, P. K. 2009. Androgens enhance plasticity of an electric communication signal in female knifefish, Brachyhypopomus pinnicaudatus. Hormones and Behavior, 56, 264-273.

Andersson, M. 1994. Sexual Selection. Princeton, New Jersey: Princeton University Press.

Bee, M. A. \& Perrill, S. A. 1996. Responses to conspecific advertisement calls in the green frog (Rana clamitans) and their role in male-male communication. Behaviour, 133, 283-301.

Bee, M. A., Perrill, S. A. \& Owen, P. C. 2000. Male green frogs lower the pitch of acoustic signals in defense of territories: a possible dishonest signal of size? Behavioral Ecology, 11, 169-177.

Candolin, U. 2000a. Changes in expression and honesty of sexual signalling over the reproductive lifetime of sticklebacks. Proceedings of the Royal Society of London, Series B, 267, 2425-2430.

Candolin, U. 2000b. Male-male competition ensures honest signaling of male parental ability in the three-spined stickleback (Gasterosteus aculeatus). Behavioral Ecology and Sociobiology, 49, 57-61.

Candolin, U. 2000c. Increased signalling effort when survival prospects decrease: malemale competition ensures honesty. Animal Behaviour, 60, 417-422.

Curtis, C. C. \& Stoddard, P. K. 2003. Mate preference in female electric fish, Brachyhypopomus pinnicaudatus. Animal Behaviour, 66, 329-336.

Davis, E. A. \& Hopkins, C. D. 1988. Behavioural analysis of electric signal localization in the electric fish, Gymnotus carapo (Gymnotiformes). Animal Behaviour, 36, 1658-1671.

Dawkins, R. \& Krebs, J. R. 1978. Animal signals: information or manipulation. In: Behavioural Ecology: an Evolutionary Approach (Ed. by J. R. Krebs \& N. B. Davies), pp. 282-309. Oxford: Blackwell.

von der Emde, G., Schwarz, S., Gomez, L., Budelli, R. \& Grant, K. 1998. Electric fish measure distance in the dark. Nature, 395, 890-894. 
Endler, J. A. 1987. Predation, light intensity and courtship behaviour in Poecilia reticulata (Pisces: Poeciliidae). Animal Behaviour, 35, 1376-1385.

Endler, J. A. 1995. Multiple-trait coevolution and environmental gradients in guppies. Trends in Ecology \& Evolution, 10, 22-29.

Fitch, W. T. 1999. Acoustic exaggeration of size in birds via tracheal elongation: comparative and theoretical analyses. Journal of Zoology, 248, 31-48.

Fitch, W. T. \& Hauser, M. D. 2010. Unpacking 'honesty': vertebrate vocal production and the evolution of acoustic signals. In: Acoustic Communication (Ed. by A. Megela Simmons \& A. N. Popper), pp. 65-137. New York: Springer-Verlag.

Franchina, C. R. 1997. Ontogeny of the electric organ discharge and the electric organ in the weakly electric pulse fish Brachyhypopomus pinnicaudatus (Hypopomidae, Gymnotiformes). Journal of Comparative Physiology A, 181, 111-119.

Franchina, C. R. \& Stoddard, P. K. 1998. Plasticity of the electric organ discharge waveform of the electric fish Brachyhypopomus pinnicaudatus. I. Quantification of day-night changes. Journal of Comparative Physiology A, 183, 759 - 768.

Franchina, C. R., Salazar, V. L., Volmar, C. H. \& Stoddard, P. K. 2001. Plasticity of the electric organ discharge waveform of male Brachyhypopomus pinnicaudatus II. Social effects. Journal of Comparative Physiology A, 187, 45-52.

Gavassa, S., Silva, A. C. \& Stoddard, P. K. 2011. Tight hormonal phenotypic integration ensures honesty of the electric signal of male and female Brachyhypopomus gauderio. Hormones and Behavior, 60, 420-426.

Gerhardt, H. C. 1994. The evolution of vocalization in frogs and toads. Annual Review of Ecology and Systematics, 25, 293-324.

Giora, J. \& Malabarba, L. R. 2009. Brachyhypopomus gauderio, new species, a new example of underestimated species diversity of electric fish in the southern South America (Gymnotiformes: Hypopomidae). Zootaxa, 2093, 60-68.

Goldina, A., Gavassa, S. \& Stoddard, P. K. 2011. Testosterone and 11-ketotestosteone have different regulatory effects on electric communication signals of male Brachyhypopomus gauderio. Hormones and Behavior, 60, 139-147.

Grafen, A. 1990. Biological signals as handicaps. Journal of Theoretical Biology, 144, 517-546. 
Hall, M. L., Molles, L. E., Illes, A. E. \& Vehrencamp, S. L. 2009. Singing in the face of death: male banded wrens Thryophilus pleurostictus sing more to playback in their last breeding season. Journal of Avian Biology, 40, 217-224.

Hanika, S. \& Kramer, B. 1999. Electric organ discharges of mormyrid fish as a possible cue for predatory catfish. Naturwissenschaften, 86, 286-288.

Hanika, S. \& Kramer, B. 2000. Electrosensory prey detection in the African sharptooth catfish, Clarias gariepinus (Clariidae), of a weakly electric mormyrid fish, the bulldog (Marcusenius macrolepidotus). Behavioral Ecology and Sociobiology, 48, 218-228.

Hill, J. A., Enstrom, D. A., Ketterson, E. D., Nolan, V. \& Ziegenfus, C. 1999. Mate choice based on static versus dynamic secondary sexual traits in the dark-eyed junco. Behavioral Ecology, 10, 91-96.

Hopkins, C. D. 1986. Temporal structure of non-propagated electric communication signals. Brain, Behavior and Evolution, 28, 43-59.

Hopkins, C. D. 1999. Design features for electric communication. Journal of Experimental Biology, 202, 1217-1228.

Hopkins, C. D., Comfort, N. C., Bastian, J. \& Bass, A. H. 1990. Functional analysis of sexual dimorphism in an electric fish, Hypopomus pinnicaudatus, order Gymnotiformes. Brain, Behavior and Evolution, 35, 350-367.

Howard, R. D. \& Young, J. R. 1998. Individual variation in male vocal traits and female mating preferences in Bufo americanus. Animal Behaviour, 55, 1165-1179.

Hughes, M. 2000. Deception with honest signals: signal residuals and signal function in snapping shrimp. Behavioral Ecology, 11, 614-623.

Johnstone, R. A. \& Grafen, A. 1993. Dishonesty and the handicap principle. Animal Behaviour, 46, 759-764.

Johnstone, R. A., Rands, S. A. \& Evans, M. R. 2009. Sexual selection and conditiondependence. Journal of Evolutionary Biology, 22, 2387-2394.

Kodric-Brown, A. \& Brown, J. H. 1984. Truth in advertising: the kinds of traits favored by sexual selection. American Naturalist, 124, 309-323.

Kokko, H. 1997. Evolutionarily stable strategies of age-dependent sexual advertisement. Behavioral Ecology and Sociobiology, 41, 99-107. 
Lindstrom, J., Pike, T. W., Blount, J. D. \& Metcalfe, N. B. 2009. Optimization of resource allocation can explain the temporal dynamics and honesty of sexual signals. American Naturalist, 174, 515-525.

Markham, M. R. \& Stoddard, P. K. 2005. Adrenocorticotropic hormone enhances the masculinity of an electric communication signal by modulating the waveform and timing of action potentials within individual cells. Journal of Neuroscience, 25, 8746-8754.

Markham, M., Allee, S., Goldina, A. \& Stoddard, P. 2009a. Melanocortins regulate the electric waveforms of gymnotiform electric fish. Hormones and Behavior, 55, 306-313.

Markham, M. R., McAnelly, M. L., Stoddard, P. K. \& Zakon, H. H. 2009b. Circadian and social cues regulate ion channel trafficking. PLoS Biology, 7, 1-14.

Maynard Smith, J. \& Harper, D. G. C. 1995. Animal signals: models and terminology. Journal of Theoretical Biology, 177, 305-311.

Miranda, M., Silva, A. \& Stoddard, P. K. 2008. Use of space is consistent with exploded lek polygyny in the gymnotiform electric fish Brachyhypopomus pinnicaudatus. Environmental Biology of Fishes, 83, 379-389.

Moller, P. 1995. Electric Fish: History and Behavior. London: Chapman \& Hall.

Proulx, S. R., Day, T. \& Rowe, L. 2002. Older males signal more reliably. Proceedings of the Royal Society of London, Series B, 269, 2291-2299.

Reby, D. \& McComb, K. 2003. Anatomical constraints generate honesty: acoustic cues to age and weight in the roars of red deer stags. Animal Behaviour, 65, 519-530.

Rohwer, S. \& Ewald, P. W. 1981. The cost of dominance and advantage of subordination in a badge signaling system. Evolution, 35, 441-454.

Ryan, M. J. 1980. Female mate choice in a Neotropical frog. Science, 209, 523-525.

Ryan, M. J. 1985. The Túngara Frog. Chicago: University of Chicago Press.

Salazar, V. K. 2009. The effect of male-male competition and its underlying regulatory mechanisms on the electric signal of the gymnotiform fish Brachyhypopomus gauderio. Ph.D. thesis, Florida International University.

Salazar, V. L. \& Stoddard, P. K. 2008. Sex differences in energetic costs explain sexual dimorphism in the circadian rhythm modulation of the electrocommunication 
signal of the gymnotiform fish Brachyhypopomus pinnicaudatus. Journal of Experimental Biology, 211, 1012-1020.

Salazar, V. L. \& Stoddard, P. K. 2009. Social competition affect electric signal plasticity and steroid hormone levels in the gymnotiform fish Brachyhypopomus gauderio. Hormones and Behavior, 56, 399-409.

Scudamore, R. E. \& McGregor, P. K. 1993. Approach paths of electric fish to an active electrode are affected by playback stimulus. Animal Behaviour, 46, 1240-1242.

Searcy, W. A. \& Nowicki, S. 2005. The Evolution of Animal Communication: Reliability and Deception in Signaling Systems. Princeton, New Jersey: Princeton University Press.

Shieh, K. T., Wilson, W., Winslow, M., McBride, D. W. \& Hopkins, C. D. 1996. Shortrange orientation in electric fish: an experimental study of passive electrolocation. Journal of Experimental Biology, 199, 2383-2393.

Silva, A., Quintana, L., Galeano, M., Errandonea, P. \& Macadar, O. 1999. Water temperature sensitivity of EOD waveform in Brachyhypopomus pinnicaudatus. Journal of Comparative Physiology A, 185, 187-197.

Silva, A., Quintana, L., Ardanaz, J. L. \& Macadar, O. 2002. Environmental and hormonal influences upon EOD waveform in gymnotiform pulse fish. Journal of Physiology-Paris, 96, 473-484.

Silva, A., Quintana, L., Galeano, M. \& Errandonea, P. 2003. Biogeography and breeding in gymnotiformes from Uruguay. Environmental Biology of Fishes, 66, 329-338.

Silva, A., Perrone, R. \& Macadar, O. 2007. Environmental, seasonal, and social modulations of basal activity in a weakly electric fish. Physiology \& Behavior, 90, 525-536.

Silva, A., Zubizarreta, L. \& Costa, G. 2010. Interspecific differences in agonistic behavior and its serotonergic modulation. In: International Congress of Neuroethology. Salamanca, Spain.

Stoddard, P. K. 1999. Predation enhances complexity in the evolution of electric fish signals. Nature, 400, 254-256.

Stoddard, P. K. 2002. Electric signals: predation, sex, and environmental constraints. Advances in the Study of Behavior, 31, 201-242. 
Stoddard, P. K. \& Markham, M. R. 2008. Signal cloaking by electric fish. BioScience, 58, 415-425.

Stoddard, P. K. \& Salazar, V. L. 2011. Energetic cost of communication. Journal of Experimental Biology, 214, 200-205.

Stoddard, P. K., Rasnow, B. \& Assad, C. 1999. Electric organ discharges of the gymnotiform fishes: III. Brachyhypopomus. Journal of Comparative Physiology A, 184, 609-630.

Stoddard, P. K., Markham, M. R. \& Salazar, V. L. 2003. Serotonin modulates the electric waveform of the gymnotiform electric fish Brachyhypopomus pinnicaudatus. Journal of Experimental Biology, 206, 1353-1362.

Stoddard, P. K., Zakon, H. H., Markham, M. R. \& McAnelly, L. 2006. Regulation and modulation of electric waveforms in gymnotiform electric fish. Journal of Comparative Physiology A, 192, 613-624.

Stoddard, P. K., Markham, M. R., Salazar, V. L. \& Allee, S. 2007. Circadian rhythms in electric waveform structure and rate in the electric fish Brachyhypopomus pinnicaudatus. Physiology \& Behavior, 90, 11-20.

Torres, R. \& Velando, A. 2003. A dynamic trait affects continuous pair assessment in the blue-footed booby, Sula nebouxii. Behavioral Ecology and Sociobiology, 55, 6572.

Wagner, W. E. 1989a. Fighting, assessment, and frequency alteration in Blanchard's cricket frog. Behavioral Ecology and Sociobiology, 25, 429-436.

Wagner, W. E. 1989b. Social correlates of variation in male calling behavior in Blanchard's cricket frog, Acris crepitans blanchardi. Ethology, 82, 27-45.

Wagner, W. E. 1992. Deceptive or honest signalling of fighting ability? A test of alternative hypotheses for the function of changes in call dominant frequency by male cricket frogs. Animal Behaviour, 44, 449-462.

Wiley, R. H. 1994. Errors, exaggeration, and deception in animal communication. In: Behavioral Mechanisms in Evolutionary Ecology (Ed. by L. A. Real), pp. 157189. Chicago: University of Chicago Press.

Wong, B. \& Svensson, P. 2009. Strategic male signalling effort in a desert-dwelling fish. Behavioral Ecology and Sociobiology, 63, 543-549. 
Zahavi, A. 1975. Mate selection: a selection for a handicap. Journal of Theoretical Biology, 53, 205-214.

Zahavi, A. 1977. The cost of honesty: further remarks on the handicap principle. Journal of Theoretical Biology, 67, 603-605. 
(a)

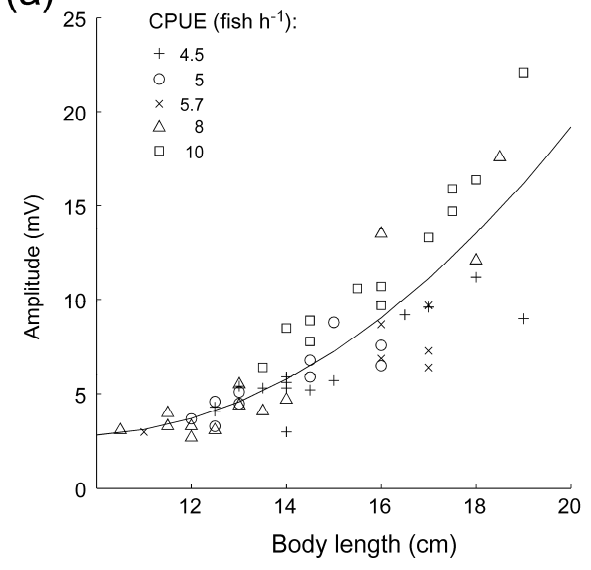

(b)

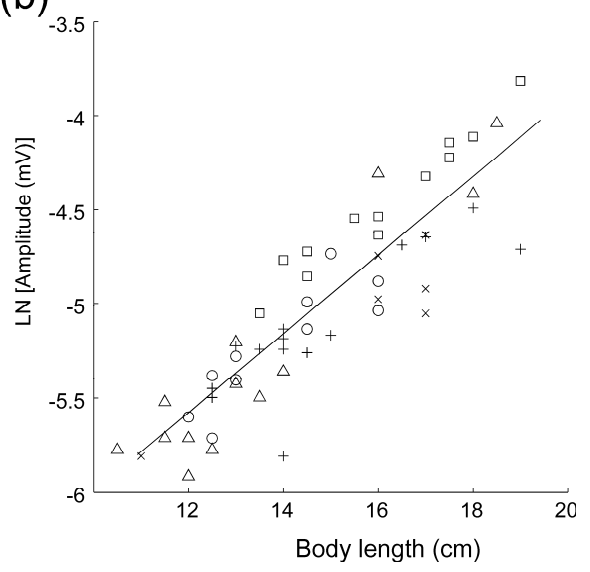

Figure 2.1. Relation between electric organ discharge (EOD) amplitude and body length in electric fish Brachyhypopomus gauderio. (a) Body length is plotted against signal amplitude in a linear scale. (b) Body length is plotted against the natural logarithm of EOD amplitude. CPUE is 'catch per unit effort', our index of population density in the field. 

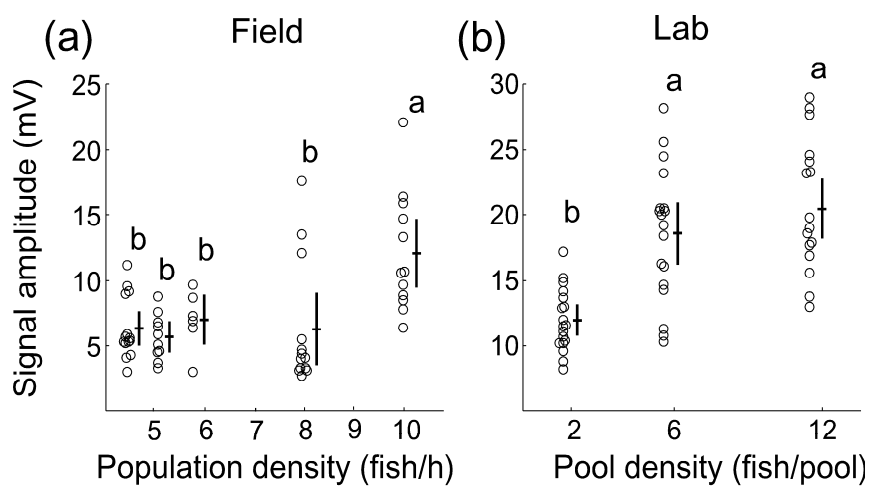

Figure 2.2. Relation between signal amplitude and population density of electric fish Brachyhypopomus gauderio under (a) natural field conditions (b) experimentally controlled laboratory conditions. Circles indicate raw data; crosses indicate means $\pm \mathrm{SE}$. Significant differences between groups, based on Tukey post hoc analysis $(\alpha=0.05)$, are noted by lower case letters. 
Field

4.5 fish $\mathrm{h}^{-1}$

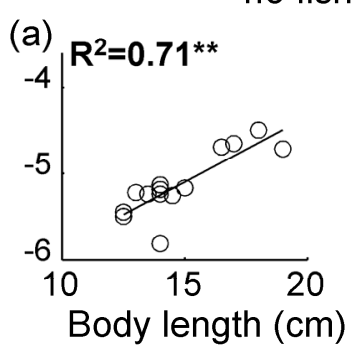

(f)

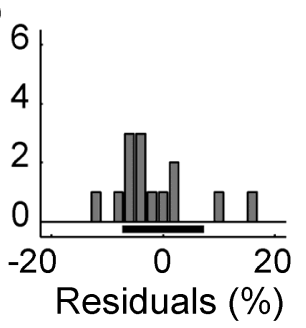

5 fish $\mathrm{h}^{-1}$

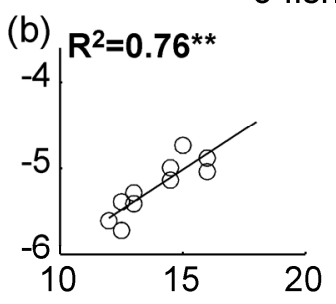

(g) 6

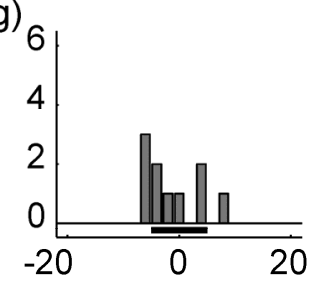

5.7 fish $\mathrm{h}^{-1}$

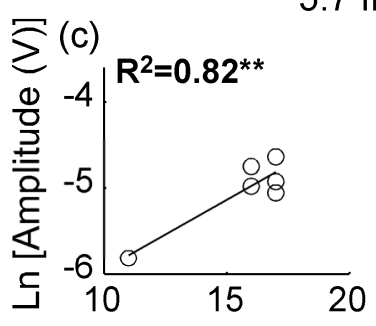

(h)

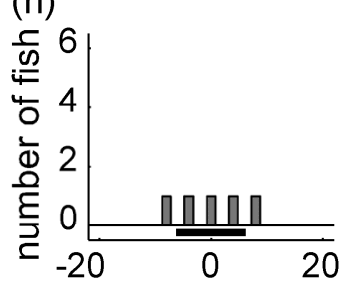

8 fish $\mathrm{h}^{-1}$

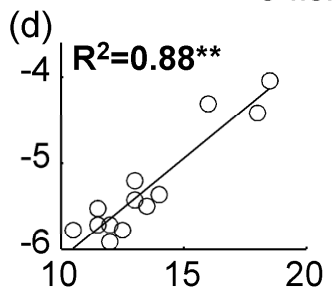

(i)

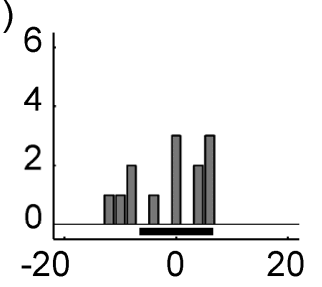

10 fish $h^{-1}$

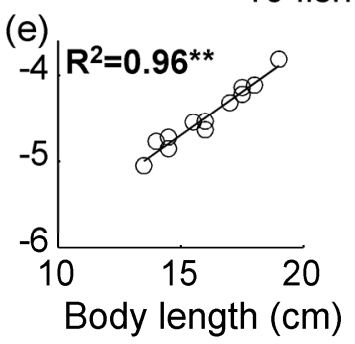

(j)

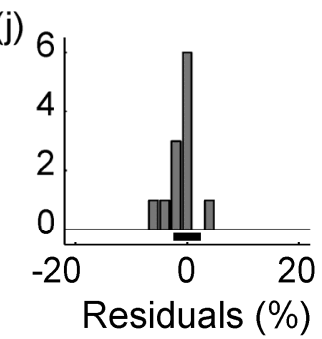

Lab

2 fish pool-1

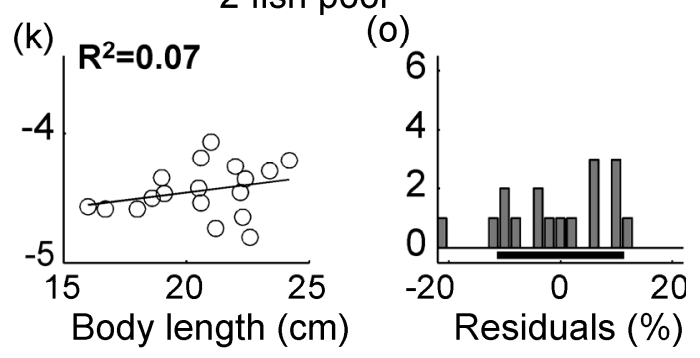

6 fish pool-1
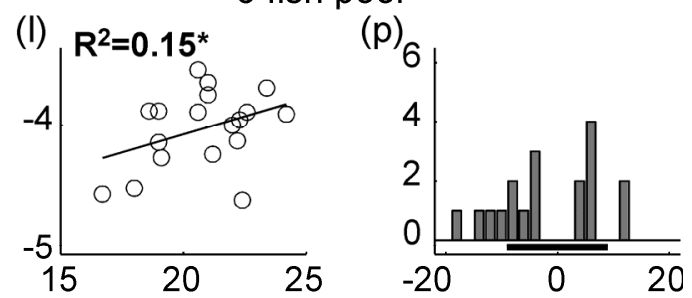

12 fish pool-1

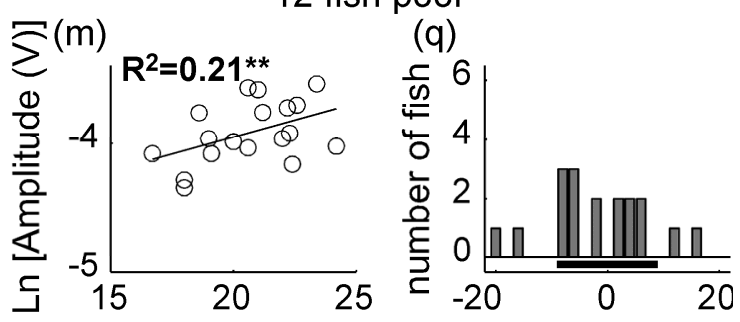

18 fish pool-1

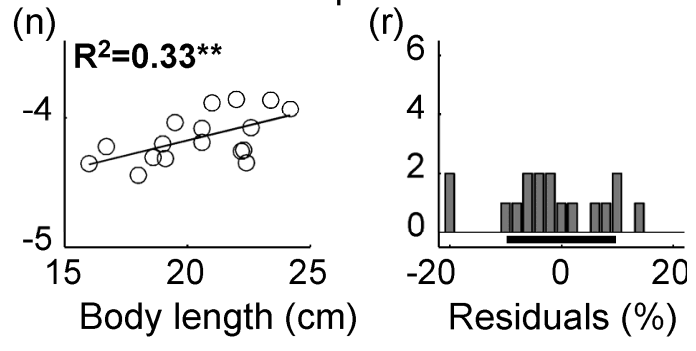

(s)

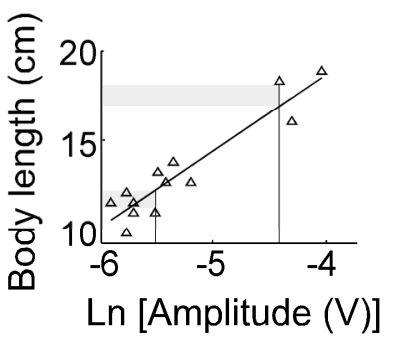

Figure 2.3. Relation between signal amplitude, body length and density of

Brachyhypopomus gauderio in the field and in the laboratory. Scatterplots and linear 
regressions show relationships between body length and the natural logarithm of signal amplitude in the field (a: $N=14, P=0.0002 ; \mathrm{b}: N=10, P=0.001 ; \mathrm{c}: N=6, P=0.013$; d: $N=13, P<0.0001 ; \mathrm{e}: N=12, P<0.0001)$ and in the laboratory $(\mathrm{k}: N=18, P=0.3 ; 1: N$ $=18, P=0.10 ; \mathrm{m}: N=18, P=0.05 ; \mathrm{n}: N=17, P=0.01) .{ }^{*} P \leq 0.1 ; * * P \leq 0.05$. Bar plots show the accuracy of EOD amplitude as a predictor of body length in the field $(f-j)$, and in the laboratory (o-r). The $X$-axis values represent the accuracy of EOD amplitude as a predictor of body length, calculated from the residuals of the regression between $\ln (\mathrm{EOD}$ amplitude) and body length (s), expressed as a percentage of body length. The height of the bars indicates the number of fish whose length could be predicted from the EOD at a given accuracy level. Horizontal bars indicate \pm SD accuracy of the EOD amplitude as a predictor of body length. Note that the accuracy of EOD amplitude as a predictor of body length was within $2.4 \%$ of the fish's actual body length (j) for the highest population density in the field (e).
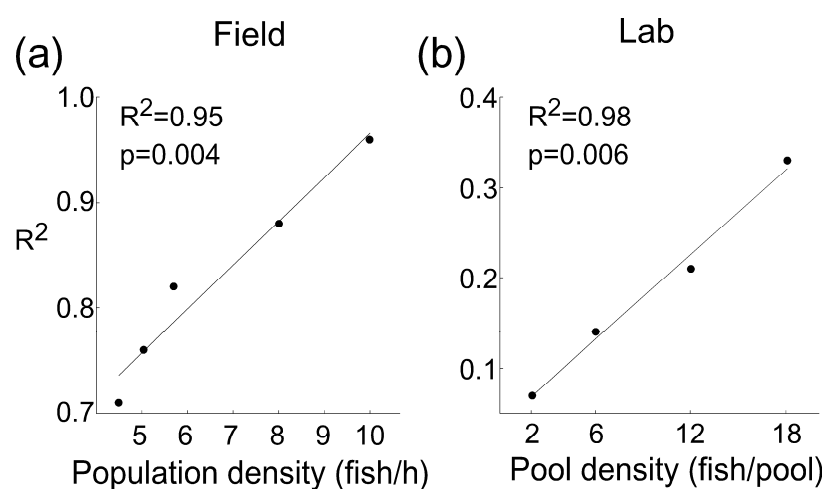

Figure 2.4. Reliability of electric organ discharge (EOD) of Brachyhypopomus gauderio as a predictor of body length at different population densities in (a) the field and (b) the laboratory. Linear regression of $R^{2}$ obtained from the linear regressions between body length and $\ln ($ EOD amplitude) (Fig. $3 \mathrm{a}-\mathrm{e}, \mathrm{k}-\mathrm{n}$ ) plotted against fish density. 
(a)

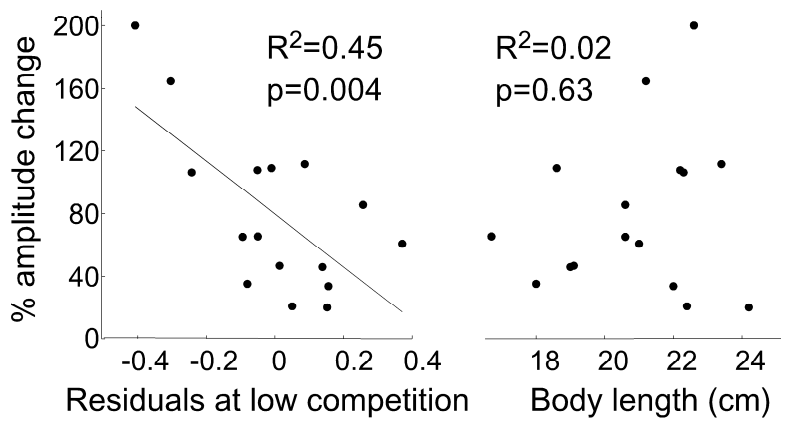

Figure 2.5. (a) Percentage change in electric organ discharge (EOD) amplitude of male Brachyhypopomus gauderio between low competition ( 2 fish/pool) and high competition (12 fish/pool) in the laboratory relative to that predicted based on male body length at low competition. The significant negative slope shows that the farther below their predicted signal amplitude at low competition (negative residuals of regression between body length and $\ln (\mathrm{EOD}$ amplitude)), the greater the males increased their signal amplitudes under high competition. (b) Relation between body length and percentage change in signal amplitude. 


\section{CHAPTER 3}

\section{TIGHT HORMONAL PHENOTYPIC INTEGRATION ENSURES HONESTY OF THE}

ELECTRIC SIGNAL OF MALE AND FEMALE BRACHYHYPOPOMUS GAUDERIO

As published in Hormones and Behavior

July 2011

Volume 60, Pages 420-426

Copyright (C) Elsevier 


\begin{abstract}
Hormones mediate sexually selected traits including advertisement signals. Hormonal co-regulation links the signal to other hormonally-mediated traits such that the tighter the integration, the more reliable the signal is as a predictor of those other traits. Androgen administration increases the duration of the communication signal pulse in both sexes of the electric fish Brachyhypopomus gauderio. To determine whether the duration of the signal pulse could function as an honest indicator of androgen levels and other androgen-mediated traits, we measured the variation in sex steroids, signal pulse duration, and sexual development throughout the breeding season of B. gauderio in marshes in Uruguay. Although the sexes had different hormone titres and signal characteristics, in both sexes circulating levels of the androgens testosterone (T) and 11ketotestosterone (11-KT) were strongly related to signal pulse duration. Consequently, signal pulse duration can serve as an honest indicator of circulating androgens in males and females alike. Additionally, through phenotypic integration, signal pulse duration also predicts other sexual traits directly related to androgen production: gonad size in males, and estradiol (E2) levels in females. Our findings show that tight hormonal phenotypic integration between advertisement signal and other sex steroid-mediated traits renders the advertisement signal an honest indicator of a suite of reproductive traits.
\end{abstract}




\section{Introduction}

Sex steroids coordinate broad suites of physiological and behavioural traits necessary for reproduction (Wingfield et al., 1990). Of these, androgens in particular regulate the expression of signals relevant for sexual communication by changing structures and activity in neural circuits (Moore et al., 2005; Ball et al., 2008; Bass, 2008; Godwin, 2010). A largely unresolved question is whether communication signals that are regulated by sex steroids can accurately reflect sex steroid levels themselves as well as other behaviourally relevant traits also regulated by sex steroids, which are not directly related to the signal. The signal could predict other sex steroid-mediated traits through second order relationships between the signal and the advertised traits that arise from first order relationships between sex steroids and traits, including the communication signal. Sex steroids would serve as intermediaries of these higher order relationships. However, phenotypic integration can be masked or impaired by differences in tissue sensitivity, in receptor density or binding affinity, in the time scale of the response to hormone levels, the plasticity of the signal, and the intervention of other hormonal regulators, any of which can compromise the reliability of the signal (Adkins-Regan, 2008; Ball et al., 2008; Kempenaers et al., 2008; Karubian et al., 2011). The central question of this study is whether the integration of the communication signal and its hormonal regulator (in this case androgens) is sufficiently tight to make the signal a reliable predictor of instantaneous endocrine and reproductive states.

Animals produce communication signals that advertise their quality to potential mates or their fighting ability to potential competitors (Searcy and Nowicki, 2005). In 
both contexts the signaller could benefit from exaggeration, but a high prevalence of cheating would turn the signals meaningless to receivers and the communication system would degrade and possibly vanish (Johnstone and Grafen, 1993). Signal honesty, on the other hand, might be stable under a select group of circumstances: when the expression of the advertisement signal depends on a sexually selected phenotypic trait of the signaller (Maynard Smith and Harper, 1995), when signals are expensive to produce or maintain, or when high quality males receive greater benefit from signalling than low quality males (Zahavi, 1977; Grafen, 1990). A related mechanism to maintain honest signalling is through hormonal control of suites of characters, of which at least one character is costly (Folstad and Karter, 1992). Hormones such as sex steroids can regulate communication signals as well as life-history traits that trade-off against one another, such as mate attraction versus parental care and immunocompetence (Wingfield et al., 1990; Casto et al., 2001; Hau, 2007). Sexually selected signals that are regulated by these same hormones should be honest due to the cost or trade-offs associated with elevated hormone levels (Folstad and Karter, 1992; Owen-Ashley et al., 2004; McGlothlin et al., 2008). Signallers could escape the cost of elevated hormone levels by increasing sensitivity of the advertisement signal to the regulating hormone or by uncoupling signal expression from the circulating hormone entirely (Ketterson and Nolan, 1999; Hau, 2007; AdkinsRegan, 2008; Ketterson et al., 2009). The success of hormonally controlled suites at assuring signal honesty will depend entirely on the tightness of phenotypic integration between circulating hormone levels and the traits they influence, at the levels of both the individual and the population (McGlothlin and Ketterson, 2008; Ketterson et al., 2009). Tight hormonal phenotypic integration guarantees that a communication signal will 
reliably indicate hormonal levels as well as upstream and downstream hormone-mediated traits, allowing signal receivers to make rapid and accurate comparisons of endocrine state and inferences of reproductive condition among signalling individuals in a population (Fig. 1).

The electric fish, Brachyhypopomus gauderio (Giora and Malabarba, 2009), sister species of B. pinnicaudatus (Hopkins et al., 1990), produces a biphasic pulse-type electric organ discharge (EOD, Fig. 2) used for active electrolocation and communication during their nocturnal active phase, as is typical of weakly electric fish (Moller, 1995). The male EOD waveform is greater in amplitude and $2^{\text {nd }}$ phase duration than the female EOD (Hopkins et al., 1990; Franchina and Stoddard, 1998). Both sexes increase the amplitude and $2^{\text {nd }}$ phase duration of the EOD at night when active (Franchina and Stoddard, 1998; Stoddard et al., 2007) and as intrasexual competition intensifies (Franchina and Stoddard, 1998; SG unpublished; Salazar and Stoddard, 2009). Socially induced enhancements of EOD parameters are accompanied by increases in testosterone $(\mathrm{T})$ and 11ketotestosterone (11-KT) (Salazar and Stoddard, 2009). In both sexes, androgen treatments mimic the effects of prolonged social interaction on the duration of EOD (Silva et al., 1999; Allee et al., 2009; Goldina et al., 2011). However, the reliability of $2^{\text {nd }}$ phase duration as an indicator of androgen level could be compromised by the strong and rapid enhancing effects of melanocortins on waveform $2^{\text {nd }}$ phase duration (Markham and Stoddard, 2005; Stoddard et al., 2006) or the interaction between androgens and melanocortins (Goldina et al., 2011). On the other hand, extending the duration of the $2^{\text {nd }}$ phase of the EOD significantly increases energetic expense (Salazar and Stoddard, 2008) 
and may increase predation risk (Stoddard, 1999). Additionally, extension of the $2^{\text {nd }}$ phase through increasing androgen titres may impose a fitness cost by compromising immunocompetence (Folstad and Karter, 1992).

The effect of experimental androgen treatment on EOD duration, the costs associated with extending EOD $2^{\text {nd }}$ phase duration, and the known effects of androgens in vertebrate reproductive physiology and behaviour, collectively prompted us to predict

that EOD $2^{\text {nd }}$ phase duration had the capacity to serve as an honest indicator of androgen levels and androgen-mediated traits in B. gauderio. However, because so many mechanisms can weaken the relationship between hormone titers and trait expression, it is uncertain whether the signal could indicate other hormonally-mediated traits (AdkinsRegan, 2008). To determine the strength of phenotypic integration between signals, steroids, and reproductive condition in B. gauderio, we estimated the relationship between androgen levels ( $\mathrm{T}$ and $11-\mathrm{KT}$ ), $2^{\text {nd }}$ phase duration (parameterized as $t_{\mathrm{P} 2}$, the time constant of repolarisation, Fig. 2), and gonadosomatic index (GSI) in a natural population that we followed throughout the breeding season.

\section{Materials and methods}

We sampled B. gauderio during the breeding season in Laguna Lavalle ( $32^{\circ} 01.259^{\prime}$ $\left.\mathrm{S}, 055^{\circ} 22.498^{\prime} \mathrm{W}\right)$ in Department of Tacuarembó, Uruguay, Oct-Dec 2009. Water conductivity was $38-57 \mu \mathrm{S} \mathrm{cm}^{-1}$, and temperature was $19-28^{\circ} \mathrm{C}$ throughout the study. All sampling took place during the day (11:00-17:59 UYST) when the fish are inactive in 
their refuges among the plant roots. All sampling was conducted at sites with similar depth, distance to shore, and vegetation. In total we sampled 42 sexually mature females and 31 sexually mature males. Female body length ranged from 11.9 to $17.5 \mathrm{~cm}$ and body weight ranged from 4.0 to $13.0 \mathrm{~g}$. Male body length ranged from 11.2 to $19.3 \mathrm{~cm}$ and body weight ranged from 5.1 to $12.0 \mathrm{~g}$.

Fish were located using an audio amplifier to convert their electric signals into sound, netted rapidly, and taken into a floating EOD recording rig. EODs were recorded within a minute of disturbing the fish, before melanocortin-mediated EOD waveform modulation can take effect (Stoddard et al., 2006). Immediately after recording the EOD, fish were anesthetized by immersion in a fast-acting eugenol solution $\left(1.2 \mathrm{mg} \mathrm{l}^{-1}\right)$ for blood sampling. In the lab we have observed that taking the fish out of the water and injecting it, even with saline, causes a rapid drop in EOD amplitude. However, it quickly recovers after the fish is returned to its tank (Stoddard, 2007). On the other hand, steroids take several minutes before noticeable changes in the circulating levels can be observed. We took the blood samples within the time range usually used for lab studies in gymnotiforms (Dunlap, 2002; Salazar and Stoddard, 2008).

After blood sampling, fish were then euthanized in a stronger eugenol solution (8 $\mathrm{mg}^{-1}$ ) and fixed in $10 \%$ formalin. We weighed and measured the fish, and noted whether the tail was intact, since tail damage changes the EOD waveform (Hopkins et al., 1990). In this genus it is almost impossible to accurately remove all gonad tissue from fresh samples. Therefore, it is typically done in fixed specimens (Schaan et al., 2009). After 5 days of fixation, we determined sex by gonadal inspection and weighed the gonads to 
calculate the gonadosomatic index (GSI) [100*gonad weight / body weight]. Collections and experimental procedures were performed under the guidelines and approval of the FIU IACUC (08-027).

\section{EOD measurements}

Field EOD recordings took place inside the lagoon in a submerged plastic sheet cage $(100 \times 50 \times 50 \mathrm{~cm})$ with recording electrodes located at either end, $100 \mathrm{~cm}$ apart. A mesh tube held the fish lengthwise, equidistant from the recording electrodes and $25 \mathrm{~cm}$ below the water surface. A ground electrode was located perpendicular to the fish. EODs were differentially amplified 100X (World Precision Instruments Inc., Sarasota, FL. DAM-50, AC-coupled, high pass filter corner $0.1 \mathrm{~Hz}$, low pass filter corner $10 \mathrm{kHz}$ ). Signals were digitized by an RM1 mobile processor (Tucker Davis Technologies, Alachua, FL), and stored and analyzed on a portable computer using custom software developed in MATLAB. We analyzed the EODs to calculate amplitude and second phase duration, parameterized as $t_{\mathrm{p} 2}$, the time constant of an inverse exponential function fitted to the repolarisation curve of the second phase between $30 \%$ and $70 \%$ of the amplitude (Fig. 2).

Blood collection and hormone assays

After recording the EOD, and within four minutes of capture, we drew blood from the ventral vertebral sinus into a syringe containing $5 \mu 1$ 10\% EDTA. Sample handling and androgen extraction and quantification ( $\mathrm{T}$ and 11-KT) followed a validated protocol for this species (Salazar and Stoddard, 2009). In females we assayed E2 as well. Plasma was diluted 1:12.5 in buffer provided with the immunoassay kit (Cayman Chemical, Inc., 
Ann Arbor, MI). For the assay, $250 \mu \mathrm{l}$ of diluted plasma was extracted four times in 4:1 hexane:ethyl acetate solution, solvent was evaporated in a vacuum centrifuge, and the sample resuspended in immunoassay buffer. Because we were short on plasma after the androgen assays, and the sensitivity of the E2 assay was not high enough for the volume of plasma we had remaining, we biased sample E2 concentrations into the dynamic range of the EIA by spiking the samples with E2 standard provided with the kit. The sensitivity of the E2 assay (19 $\mathrm{pg} / \mathrm{ml})$ is low compared to the sensitivity of T $(6 \mathrm{pg} / \mathrm{ml})$ and $11-\mathrm{KT}$ $(1.3 \mathrm{pg} / \mathrm{ml})$, for which we could dilute the female plasma 1:50 and 1:12, respectively, and still be within the dynamic range of the assay. However for E2, a dilution of 1:12, which was the highest concentration we could get with the amount of plasma left and the volume required by the EIA kit, was below the dynamic range of the assay for some samples. Thus, we had to spike the samples with E2 to be sure. All samples were plated in duplicate as specified by the kit manufacturer. To verify extraction recovery, we extracted EIA kit standards alongside the plasma samples. Sample concentrations were corrected for EDTA addition, dilution, and percentage of recovery. Extraction of $\mathrm{T}$ and $11-\mathrm{KT}$ were run in three batches, with a recovery percentage of $\mathrm{T}: 98.9 \%, 98.1 \%$, and $96.9 \%$ each, and $93.1 \%, 97.7 \%$, and $94.4 \%$ for $11-\mathrm{KT}$. E2 extractions were run in two batches, with a recovery percentage of $95.8 \%$ and $91.9 \%$ each. We ran samples in six T assays, five 11-KT assays, and two E2 assays and calculated the coefficient of variation from pooled samples. For T, the intra-assay coefficients of variation were 5.37\%, 5.61\%, $8.5 \%, 2.3 \%, 4.5 \%$ and $4.96 \%$ and the inter-assay coefficient of variation was $14.58 \%$. For $11-\mathrm{KT}$, the intra-assay coefficients of variation were $6.69 \%, 1.67 \%, 2.96 \%, 6.26 \%$ and $0.95 \%$, while the inter-assay coefficient of variation was $3.51 \%$. For E2, the intra-assay 
coefficients of variation were $6.46 \%$ and $4.48 \%$, while the inter-assay coefficient of variation was $7.07 \%$.

\section{Data analyses}

We normalized all the variables with a natural log transform, verified with Shapiro-Wilk test. We selected an appropriate regression model to test the relationships among steroid levels, signal duration, and gonad maturation, and tested the fit of the regression models using residual analysis. Prior studies used linear regressions on raw data between steroid levels and EOD parameters (Salazar and Stoddard, 2009). However, our large data set allowed us to do residual analysis of the fit of the regression, as explained above. The residual analysis showed that a linear regression on raw data was not the best fit since it violated the assumption of heteroskedasticity, and the residuals were still correlated with the predicting variable, indicating a higher order relationship. We performed an analysis of covariance to test whether the linear regressions differed between the sexes. Significant differences were then tested for differences in slope and intercept using Fisher's LSD post hoc test. All statistical analyses were performed using the MATLAB Statistical Toolbox, setting $\alpha=0.05$. Analyzing $11-\mathrm{KT}$ or $\mathrm{T}$ by themselves does not give a reliable picture since both androgens act on the EOD waveform in males (Goldina et al., 2011) and individuals may have a different ratio of T to 11-KT. Thus, we had to construct a model to combine both androgens and take into account both androgens simultaneously. We know from a prior lab study that $11-\mathrm{KT}$ is 1.15 times more potent than $\mathrm{T}$ in increasing EOD $\mathrm{t}_{\mathrm{P} 2}$ in males (Goldina et al., 2011), we assumed the 
same was true for females, although no studies have addressed this issue. Therefore, our combined model weighted 11-KT 1.15 times over T.

\section{Results}

$\underline{\text { Sex differences in } \mathrm{t}_{\mathrm{p} 2} \text { and androgen levels }}$

Males had greater EOD tp2 than females $(\mathrm{p}<0.001, \mathrm{t}=-6.10, \mathrm{df}=59$, Fig. 3a). Males also had slightly greater mean circulating levels of testosterone than females but the ranges overlapped considerably. Males had much greater circulating levels of 11ketotestosterone than females (ANOVA, $\mathrm{F}_{3,95}=96.5$, $\mathrm{p}<0.001$, Fig. $3 \mathrm{~b}$ ). Moreover, males had similar levels of 11-KT and $\mathrm{T}$, while females had significantly higher levels of $\mathrm{T}$ than 11-KT. In both sexes the levels of 11-KT and T were strongly related (Table 1, Fig. 4), although the slopes of these relationships did not differ between the sexes, the intercepts were significantly different (ANCOVA, $\mathrm{F}_{\text {stat }}=0.81$, d.f. $=32$, slope $\mathrm{p}=0.37$, intercept $\mathrm{p}<0.001)$.

$\underline{\text { Signal duration as an honest indicator of androgen levels }}$

Plasma levels of T and 11-KT were both highly correlated with EOD pulse duration ( $t_{\mathrm{P} 2}$ parameter) in the field population (Table 1). However, the relationship between $\mathrm{T}$ levels and tp2 was steeper in males than in females, probably because males also have greater 11-KT acting on $\mathrm{t}_{\mathrm{P} 2}$ than females (Table $1 \&$ Supplementary Table 1; T: ANCOVA, $\mathrm{F}=4.8, \mathrm{df}=42$, slope $\mathrm{p}=0.034$, intercept $\mathrm{p}<0.001$; 11-KT: ANCOVA, $\mathrm{F}=1.01$, $\mathrm{df}=32$, slope $\mathrm{p}=0.32$, intercept $\mathrm{p}=0.53$ ). Because both androgens are biologically active in 
regulating the waveform in males (Goldina et al., 2011), we used a weighted sum of these two androgens as the independent variable in our regression model, weighting 11-KT plasma titres by 1.15 relative to $T$. Under the weighted model, the relationship between $t_{\mathrm{P} 2}$ and androgen levels remained significant in males but it was lost in females (Table 1, Fig. 5). Our combined model is not better nor worse than using only $11-\mathrm{KT}$ to explain $\mathrm{t}_{\mathrm{P} 2}$ variation in males $\left(\mathrm{F}_{1,18}=0.224, \mathrm{p}=0.64\right)$, but it is our best attempt to account for both androgens acting on the EOD waveform. Although $t_{\mathrm{P} 2}$ can serve as a reliable indicator of the circulating levels of $\mathrm{T}$ and 11-KT in both sexes, the combined model may not apply to females as we constructed it, or the combined relationship suffered from a reduced sample size, since we had very few females for which we had enough plasma to measure both androgens.

Phenotypic integration and the information conveyed by the electric signal

In males but not in females, EOD $t_{\mathrm{P} 2}$ predicted GSI, a $2^{\text {nd }}$ order relationship (males: Table 1, Fig. 6a). Androgens should be the logical mechanistic linkage between GSI and EOD t $t_{\mathrm{P} 2}$. Accordingly, we found a significant relationship between GSI and both T and 11-KT levels in males, but neither androgen nor E2 was related to GSI in females (Table $1)$.

T levels predicted E2 levels in females (Table 1), a $1^{\text {st }}$ order relationship since E2 is synthesized from T. EOD t $t_{2}$ also predicted circulating level of E2 in females, presumably a $2^{\text {nd }}$ order relationship, with $\mathrm{T}$ as the common link (Table 1, Fig. 6b). 
As a consequence of these significant $2^{\text {nd }}$ order relationships with circulating androgen levels, extension of the waveform's $2^{\text {nd }}$ phase, EOD $t_{P 2}$, can function as an honest indicator of other reproductive traits, such as gonad size in males and estradiol levels in females.

\section{Discussion}

We found tight phenotypic integration between androgen-mediated traits, which resulted in $t_{\mathrm{P} 2}$ predicting androgen levels $(\mathrm{T}, 11-\mathrm{KT})$ in both sexes, GSI in males, and E2 in females. Under tight phenotypic integration, a strong correlation between androgen levels and androgen-mediated traits would allow any one of those traits to serve as an honest indicator of the others. The tight relationships we have found between androgens, signal structure, reproductive condition, and other steroids in B. gauderio indicate that the $2^{\text {nd }}$ phase duration of the electric signal waveform can serve as a reliable intraspecific signal of circulating androgen levels, as well as expression levels of androgen-mediated traits. Through phenotypic integration, EOD t $t_{\mathrm{P} 2}$ can serve as an honest indicator of traits not directly involved in signal regulation, such as gonad size in males and E2 levels in

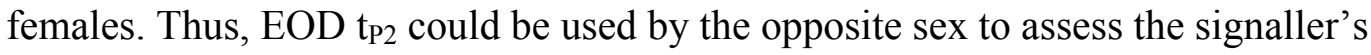
reproductive condition. By assessing male gonad size from the electric signal, females might benefit by being able to identify males with mature gonads that would fertilize their eggs with greater reliability. The information conveyed by EOD $t_{\mathrm{P} 2}$ remains honest

since the hormones (androgens) that regulate its expression also regulate the traits that EOD $t_{\mathrm{P} 2}$ is advertising. 
The relationship between EOD $t_{\mathrm{P} 2}$ and GSI may originate from the action of sex steroids in gonadal development. In teleost fish, 11-KT is released by testicular Leydig cells and acts on adjacent Sertoli cells to induce maturation of spermatogonia (Nagahama, 1994; Devlin and Nagahama, 2002). Nonetheless, gonadal development in fish requires a complex interplay between brain gonadotropin-releasing hormone, pituitary gonadotropins, and gonadal and non-gonadal sex steroids (Devlin and Nagahama, 2002; Zohar et al., 2010). Thus, other sex hormones upstream of sex-steroids such as gonadotropins and gonadotropin-releasing hormone can also contribute to the integration between EOD t $\mathrm{P}_{\mathrm{p}}$, steroid levels, and gonadal size.

In females, EOD t t $2_{2}$ also predicts E2 levels even though E2 probably does not increase $t_{\mathrm{p} 2}$. Long-term $\mathrm{E} 2$ treatment did not affect EOD duration of the female $B$. occidentalis (Hagedorn and Carr, 1985), a congener of our study species B. gauderio. Moreover, acute E2 injections did not affect EOD duration of male B. gauderio (PKS, unpublished). Therefore, we think the relationship between $\mathrm{t}_{\mathrm{P} 2}$ and $\mathrm{E} 2$ arises from the phenotypic integration of $\mathrm{T}$ as a precursor of $\mathrm{E} 2$ and a modulator of $\mathrm{EOD} \mathrm{t}_{\mathrm{P} 2}$ parameter. Inexplicably, though, the relationship between E2 and $t_{\mathrm{P} 2}$ was stronger than either the relationship between $\mathrm{T}$ and $\mathrm{E} 2$, or between $\mathrm{T}$ and $\mathrm{t}_{\mathrm{P} 2}$.

Ketterson et al. (2009) hypothesized that selection favouring strong phenotypic integration in both sexes should result in both sexes expressing a similar phenotype when their levels of a particular hormone are the same. The steeper slope in the relationship between $T$ and $t_{P 2}$ in males compared to females would indicate a greater sensitivity to $T$ in males. However, males also have about 100 times more circulating 11-KT than 
females (Fig. 3b \& 4), which also increases tp2 (Goldina et al., 2011). To make sense of the whole picture, we should note that females have a lot more T than 11-KT while males have more 11-KT than T (Fig. 3b \& 4). Since both androgens act on EOD waveform, plotting each androgen separately does not allow us to determine whether sex differences in signal phenotype are due to differences in tissue sensitivity or just to differences in circulating androgen levels. Unfortunately, our combined androgen model did not result in a significant relationship between androgen levels and EOD t $\mathrm{P}_{\mathrm{P} 2}$ in females, although there is a high degree of overlap between total androgen levels and EOD t $t_{2}$ in the sexes (Fig. 5). It is possible that the relative sensitivity of $\mathrm{T}$ and $11-\mathrm{KT}$ is different in females and males; future studies should address this issue. At this point we cannot determine whether the differences in EOD $t_{\mathrm{P} 2}$ between males and females are due to differences in circulating androgen levels or to differences in tissue sensitivity or receptor affinity. Females implanted with the non-aromatizable androgen 5-dihydrotestosterone increased EOD $t_{\mathrm{P} 2}$, and females with the highest implant doses produced EODs of greater $t_{\mathrm{P} 2}$ than untreated males (Allee et al., 2009). Testosterone levels in the two sexes are highly correlated in teleosts, indicating weak or nonexistent selection to decouple female testosterone production from that of males (Mank, 2007). However, the end products differ profoundly between the sexes, with male teleosts converting much of their testosterone to 11-ketotestosterone, and females converting theirs to estrogens. Thus, sexual selection appears to act on the downstream enzymatic pathways, and not on the production of testosterone per se. Therefore, the apparent extension of female EODs by testosterone may be a non-adaptive by-product of estradiol biosynthesis, in combination 
with direct selection on males for expression of androgen receptors in electrocytes causing their expression in both sexes.

Phenotypic integration among sex steroid actions allows the social signal to convey reproductive condition, and possibly serve as an honest indicator of other steroidmediated traits that we did not measure (Fig. 7). For instance, the androgen-regulated structure of the electric signal, EOD $t_{\mathrm{P} 2}$ could provide information about the signaller's dominance status and aggressive behaviour (Wingfield et al., 1990; Balthazart et al., 1996). 11-KT in particular is implicated in regulating dominance, aggression, and secondary sexual characteristics in male teleost fish (Schaefer and Zakon, 1996; Dunlap, 2002; Oliveira et al., 2005; Fernandes et al., 2010; Maruska and Fernald, 2010). Male $B$. gauderio increase EOD amplitude and duration more when interacting with males than with females (Franchina et al., 2001), while androgen-treated females increase their aggression and EOD responsiveness to female intruders but not to male intruders (Allee et al., 2009). Thus it is likely that a conspecific receiver could attend to signal duration to infer the aggressiveness of the signaller through a secondary relationship similar to those in our field data shown above.

Phenotypic integration is neither fixed nor resistant to evolution (Adkins-Regan, 2008). Phenotypic integration may arise from correlational selection favouring the coexpression of traits that work well together and thus will be maintained as long as selection continues to favour the correlations within that suite of traits (Wingfield et al., 1997; McGlothlin and Ketterson, 2008; Ketterson et al., 2009). If females screen prospective mates for reproductive condition to minimize the cost of unfertilized eggs, 
males would benefit from advertising to prospecting females their degree of reproductive investment, thus favouring tighter integration of signal and reproductive state. Dishonesty in the form of exaggerated signals may be discouraged by energetic expense (Salazar and Stoddard, 2008) and predation costs of spectral shifting from 2nd phase extension (Stoddard, 1999). Alternately, honest signalling may be sustained by evolutionary inertia caused by phenotypic integration (McGlothlin and Ketterson, 2008; Ketterson et al., 2009). In addition to advertising his reproductive condition and capability to fertilize eggs, a male could extend EOD t $t_{2}$ to advertise genetic quality by broadcasting his ability to bear an energetically expensive (Salazar and Stoddard, 2008) and potentially risky signal (Stoddard, 1999). Females, on the other hand, may not need to advertise their reproductive condition, since they do not engage courting males except when they are ready to spawn (PKS unpublished). We should also recognize that the relations found in this study between signal structure and other androgen-mediated traits account for roughly $20-40 \%$ of the variance. The remaining variance still leaves room for some degree of dishonest signalling.

\section{Acknowledgments}

We thank R. Perrone, L. Zubizarreta, G. Batista, T. de los Campos, D. Colacce, V. Gavassa I. Becerra, P. Pouso, and J. Campbell for assistance in the field. We thank Dr. O. Macadar, Ministerio de Cultura y Educación, Uruguay, and Facultad de Ciencias, Universidad de la República, Montevideo, Uruguay, for logistic assistance in the field. We thank Dr. L. Kim and Dr. F. Noriega for use of the plate reader, and A. Goldina and 
V. Salazar for assistance with immunoassays. We thank Dr. E. Ketterson and two anonymous reviewers for helpful comments on the manuscript. This work was supported by Tinker Field Research Grants (LACC at FIU) to S.G., University Graduate School's Dissertation Evidence Acquisition Fellowship to S.G., Judith Evans Scholarship for travel to S.G., and Animal Behavior Society award to S.G., and NSF grant IOS 0956603 to P.K.S.

\section{References}

Adkins-Regan, E., 2008. Review. Do hormonal control systems produce evolutionary inertia? Philos. Trans. R. Soc. Lond. B. 363, 1599-609.

Allee, S. J., Markham, M. R., Stoddard, P. K., 2009. Androgens enhance plasticity of an electric communication signal in female knifefish, Brachyhypopomus pinnicaudatus. Horm. Behav. 56, 264-273.

Ball, G. F., Tlemçani, O., Balthazart, J., 2008. Individual variation and the endocrine regulation of behaviour and physiology in birds: a cellular/molecular perspective. Philos. Trans. R. Soc. Lond. B. 363, 1699-1710.

Balthazart, J., Tlemçani, O., Ball, G. F., 1996. Do sex differences in the brain explain sex differences in the hormonal induction of reproductive behavior? What 25 years of research on the Japanese quail tells us. Horm. Behav. 30, 627-661.

Bass, A. H., 2008. Steroid-dependent plasticity of vocal motor systems: Novel insights from teleost fish. Brain Res. Rev. 57, 299-308.

Casto, J., Nolan Jr, V., Ketterson, E. D., 2001. Steroid hormones and immune function: experimental studies in wild and captive dark-eyed juncos (Junco hyemalis). Am. Nat. 157, 408-420. 
Devlin, R. H., Nagahama, Y., 2002. Sex determination and sex differentiation in fish: an overview of genetic, physiological, and environmental influences. Aquaculture. 208, 191-364.

Dunlap, K. D., 2002. Hormonal and body size correlates of electrocommunication behavior during dyadic interactions in a weakly electric fish, Apteronotus leptorhynchus. Horm. Behav. 41, 187-194.

Fernandes, C. C., Smith, G. T., Podos, J., Nogueira, A., Inoue, L., Akama, A., Ho, W. W., Alves-Gomes, J., 2010. Hormonal and behavioral correlates of morphological variation in an Amazonian electric fish (Sternarchogiton nattereri: Apteronotidae). Horm. Behav. 58, 660-668.

Folstad, I., Karter, A. J., 1992. Parasites, bright males, and the immunocompetence handicap. Am. Nat. 139, 603-622.

Franchina, C. R., Salazar, V. L., Volmar, C. H., Stoddard, P. K., 2001. Plasticity of the electric organ discharge waveform of male Brachyhypopomus pinnicaudatus II. Social effects. J. Comp. Physiol. A. 187, 45-52.

Franchina, C. R., Stoddard, P. K., 1998. Plasticity of the electric organ discharge waveform of the electric fish Brachyhypopomus pinnicaudatus. I. Quantification of day-night changes. J. Comp. Physiol. A. 183, 759 - 768.

Giora, J., Malabarba, L. R., 2009. Brachyhypopomus gauderio, new species, a new example of underestimated species diversity of electric fish in the southern South America (Gymnotiformes: Hypopomidae). Zootaxa. 2093, 60-68.

Godwin, J., 2010. Neuroendocrinology of sexual plasticity in teleost fishes. Front. Neuroendocrin. 31, 203-16.

Goldina, A., Gavassa, S., Stoddard, P. K., 2011. Testosterone and 11-ketotestosteone have different regulatory effects on electric communication signals of male Brachyhypopomus gauderio. Horm. Behav. 60, 139-147.

Grafen, A., 1990. Biological signals as handicaps. J. Theor. Biol. 144, 517-546.

Hagedorn, M., Carr, C., 1985. Single electrocytes produce a sexually dimorphic signal in South American electric fish, Hypopomus occidentalis (Gymnotiformes, Hypopomidae). J. Comp. Physiol. A. 156, 511-523. 
Hau, M., 2007. Regulation of male traits by testosterone: implications for the evolution of vertebrate life histories. Bioessays. 29, 133-144.

Hopkins, C. D., Comfort, N. C., Bastian, J., Bass, A. H., 1990. Functional analysis of sexual dimorphism in an electric fish, Hypopomus pinnicaudatus, order Gymnotiformes. Brain Behav. Evol. 35, 350-67.

Johnstone, R. A., Grafen, A., 1993. Dishonesty and the handicap principle. Anim. Behav. $46,759-764$.

Karubian, J., Lindsay, W. R., Schwabl, H., Webster, M. S., 2011. Bill coloration, a flexible signal in a tropical passerine bird, is regulated by social environment and androgens. Anim. Behav. 81, 795-800.

Kempenaers, B., Peters, A., Foerster, K., 2008. Sources of individual variation in plasma testosterone levels. Philos. Trans. R. Soc. Lond. B. 363, 1711-23.

Ketterson, E. D., Atwell, J. W., McGlothlin, J. W., 2009. Phenotypic integration and independence: Hormones, performance, and response to environmental change. Integr. Comp. Biol. 49, 365-379.

Ketterson, E. D., Nolan, V., Jr., 1999. Adaptation, Exaptation, and Constraint: A Hormonal Perspective. Am. Nat. 154, S4-S25.

Mank, J. E., 2007. The evolution of sexually selected traits and antagonistic androgen expression in actinopterygiian fishes. Am. Nat. 169, 142-149.

Markham, M. R., Stoddard, P. K., 2005. Adrenocorticotropic hormone enhances the masculinity of an electric communication signal by modulating the waveform and timing of action potentials within individual cells. J. Neurosci. 25, 8746-8754.

Maruska, K. P., Fernald, R. D., 2010. Behavioral and physiological plasticity: Rapid changes during social ascent in an African cichlid fish. Horm. Behav. 58, 230240.

Maynard Smith, J., Harper, D. G. C., 1995. Animal signals: models and terminology. J. Theor. Biol. 177, 305-311.

McGlothlin, J. W., Jawor, J. M., Greives, T. J., Casto, J. M., Phillips, J. L., Ketterson, E. D., 2008. Hormones and honest signals: males with larger ornaments elevate testosterone more when challenged. J. Evolution. Biol. 21, 39-48.

McGlothlin, J. W., Ketterson, E. D., 2008. Hormone-mediated suites as adaptations and evolutionary constraints. Philos. Trans. R. Soc. Lond. B. 363, 1611-1620. 
Moller, P., 1995. Electric Fish: history and behavior. Chapman \& Hall, London, New York.

Moore, F. L., Boyd, S. K., Kelley, D. B., 2005. Historical perspective: Hormonal regulation of behaviors in amphibians. Horm. Behav. 48, 373-83.

Nagahama, Y., 1994. Endocrine regulation of gametogenesis in fish. Int. J. Dev. Biol. 38, 217-229.

Oliveira, R. F., Ros, A. F., Goncalves, D. M., 2005. Intra-sexual variation in male reproduction in teleost fish: a comparative approach. Horm. Behav. 48, 430-9.

Owen-Ashley, N. T., Hasselquist, D., Wingfield, J. C., 2004. Androgens and the immunocompetence handicap hypothesis: Unraveling direct and indirect pathways of immunosuppression in song sparrows. Am. Nat. 164, 490-505.

Salazar, V. L., Stoddard, P. K., 2008. Sex differences in energetic costs explain sexual dimorphism in the circadian rhythm modulation of the electrocommunication signal of the gymnotiform fish Brachyhypopomus pinnicaudatus. J. Exp. Biol. 211, 1012-1020.

Salazar, V. L., Stoddard, P. K., 2009. Social competition affect electric signal plasticity and steroid hormone levels in the gymnotiform fish Brachyhypopomus gauderio. Horm. Behav. 56, 399-409.

Schaan, A. B., Giora, J., Fialho, C. B., 2009. Reproductive biology of the Neotropical electric fish Brachyhypopomus draco (Teleostei: Hypopomidae) from southern Brazil. Neotrop. Icthyol. 7, 737-744.

Schaefer, J., Zakon, H. H., 1996. Opposing actions of androgen and estrogen on in vitro firing frequency of neuronal oscillators in the electromotor system. J. Neurosci. $16,2860-8$.

Searcy, W. A., Nowicki, S., 2005. The evolution of animal communication: reliability and deception in signaling systems. Princeton University Press, Princeton, NJ, USA.

Silva, A., Quintana, L., Galeano, M., Errandonea, P., Macadar, O., 1999. Water temperature sensitivity of EOD waveform in Brachyhypopomus pinnicaudatus. J. Comp. Physiol. A. 185, 187-197.

Stoddard, P. K., 1999. Predation enhances complexity in the evolution of electric fish signals. Nature. 400, 254-256. 
Stoddard, P. K., 2007, Plasticity of the electric organ discharge waveform: contexts, mechanisms, and implications for electrocommunication. In: F. Ladich, et al., (Eds.), Communication in Fishes. Science Publisher, Inc., Enfield, NH, USA, pp. 623-646.

Stoddard, P. K., Markham, M. R., Salazar, V. L., Allee, S., 2007. Circadian rhythms in electric waveform structure and rate in the electric fish Brachyhypopomus pinnicaudatus. Physiol. Behav. 90, 11-20.

Stoddard, P. K., Rasnow, B., Assad, C., 1999. Electric organ discharges of the gymnotiform fishes: III. Brachyhypopomus. Journal of Comparative Physiology A: Neuroethology, Sensory, Neural, and Behavioral Physiology. 184, 609-630.

Stoddard, P. K., Zakon, H. H., Markham, M. R., McAnelly, L., 2006. Regulation and modulation of electric waveforms in gymnotiform electric fish. J. Comp. Physiol. A. $192,613-624$.

Wingfield, J., Hegner, R. E., Dufty Jr, A. M., Ball, G., 1990. The "challenge hypothesis": theoretical implications for patterns of testosterone secretion, mating systems, and breeding strategies. Am. Nat. 136, 829-846.

Wingfield, J. C., Jacobs, J., Hillgarth, N., 1997. Ecological constraints and the evolution of hormone-behavior interrelationships. Ann. N. Y. Acad. Sci. 807, 22-41.

Zahavi, A., 1977. The cost of honesty: further remarks on the handicap principle. J. Theor. Biol. 67, 603-605.

Zohar, Y., Munoz-Cueto, J. A., Elizur, A., Kah, O., 2010. Neuroendocrinology of reproduction in teleost fish. Gen. Comp. Endocrinol. 165, 438-455. 


\section{Table}

\begin{tabular}{|c|c|c|c|c|c|c|c|c|}
\hline \multirow[b]{2}{*}{$\begin{array}{c}\text { Log-log } \\
\text { Relationship }\end{array}$} & \multicolumn{4}{|c|}{ Males } & \multicolumn{4}{|c|}{ Females } \\
\hline & $\mathbf{R}^{2}$ & $\mathbf{p}$ & df & order & $\mathbf{R}^{2}$ & $\mathbf{p}$ & df & order \\
\hline $\mathrm{EOD} \tau_{\mathrm{P} 2}$ vs. T & 0.47 & $<0.001$ & 20 & & 0.21 & 0.02 & 24 & $1^{\mathrm{st}}$ \\
\hline $\mathrm{EOD} \tau_{\mathrm{P} 2}$ vs. $11-\mathrm{KT}$ & 0.77 & $<0.001$ & 21 & & 0.32 & 0.03 & 13 & $1^{\mathrm{st}}$ \\
\hline $\mathrm{EOD} \tau_{\mathrm{P} 2}$ vs. $[11-\mathrm{KT}+\mathrm{T}]$ & 0.73 & $<0.001$ & 19 & $1^{\text {st }}$ & 0.32 & 0.32 & 8 & $I^{s t}$ \\
\hline T vs. 11-KT & 0.65 & $<0.001$ & 25 & $1^{\text {st }}$ & 0.58 & 0.01 & 9 & $1^{\mathrm{st}}$ \\
\hline $\mathrm{EOD} \tau_{\mathrm{P} 2}$ vs. E2 & - & - & - & - & 0.45 & 0.006 & 14 & $1^{\text {st }}-2^{\text {nd }}$ \\
\hline $\mathrm{EOD} \tau_{\mathrm{p} 2}$ vs. GSI & 0.36 & 0.002 & 23 & $2^{\text {nd }}$ & $<0.01$ & 0.79 & 36 & $2^{n d}$ \\
\hline T vs. GSI & 0.13 & 0.07 & 26 & $1^{\mathrm{st}}$ & 0.02 & 0.48 & 25 & $1^{s t}$ \\
\hline 11-KT vs. GSI & 0.26 & $<0.001$ & 27 & $1^{\mathrm{st}}-2^{\mathrm{nd}}$ & 0.06 & 0.37 & 14 & $2^{\text {nd }}$ \\
\hline E2 vs. GSI & - & - & - & - & 0.019 & 0.61 & 15 & $1^{s t}-2^{n d}$ \\
\hline E2 vs. T & - & - & - & - & 0.34 & 0.035 & 12 & $1^{\mathrm{st}}$ \\
\hline
\end{tabular}

Table 3.1. Log-log relationships among sex steroid levels and steroid-mediated traits. Non-significant relationships are shown in italics. Please note that second order relationships are indirect, arising from serial ordering of first order, causal relationships. From a statistical standpoint, correlation analysis could suffice to show second order relationships, without implying causation. But, we want to show how much of the variance in the dependent variables can be inferred from the independent variable, something only regression analysis allows. For the regression equations please refer to supplementary figure 1 


\section{Figures}

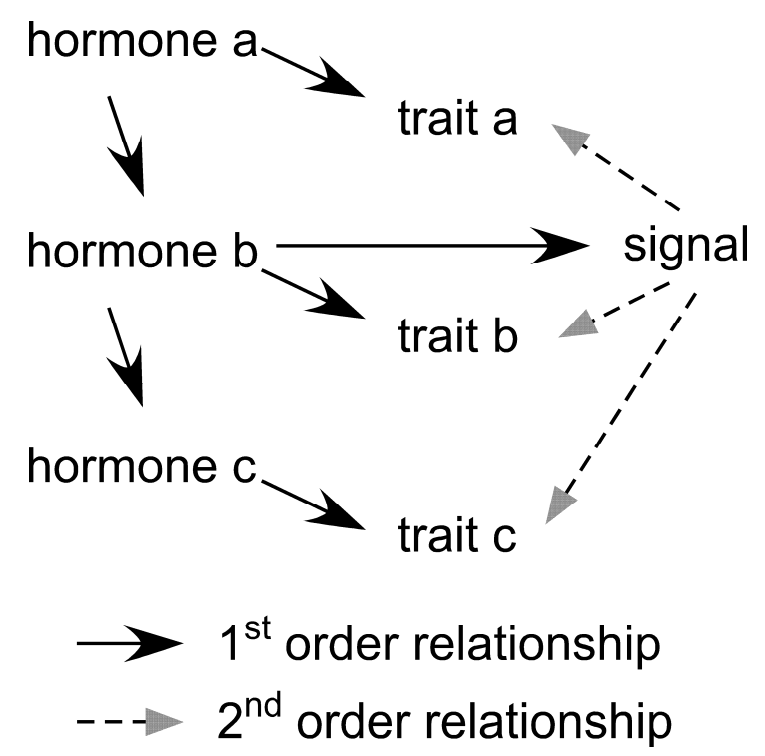

Figure 3.1. Through tight phenotypic integration, a hormonally controlled communication signal could reliably indicate circulating levels of its hormonal regulator. More importantly, under phenotypic integration, the signal could indicate other traits that are regulated by the same hormone via indirect, second order relationships. Second order relationships arise from direct first order relationships (i. e. the hormone regulating the expression of traits, such as the advertising signal). If the phenotypic integration is sufficiently tight, a receiver can use the communication signal as an external indicator of the expression of less perceptible traits. 


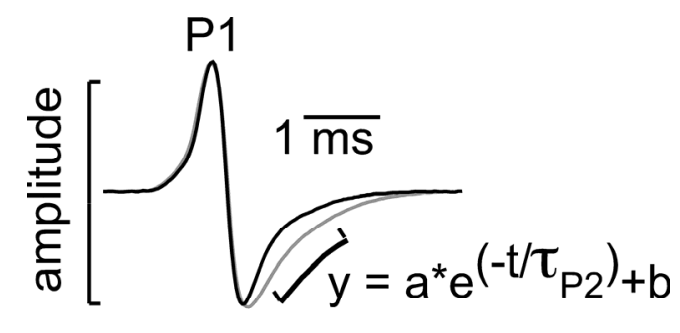

$\mathrm{P} 2$

Figure 3.2. The biphasic EOD waveform of B. gauderio varies in peak-to-peak amplitude and the duration of the $2^{\text {nd }}$ phase, as measured by EOD $t_{\mathrm{p} 2}$, the repolarization time constant of the second phase. The gray trace shows an EOD after an increase in $t_{\mathrm{p} 2}$ parameter. The asymmetric extension of the second phase is important to perception because it shifts energy to the low frequency spectrum (Stoddard et al., 1999).
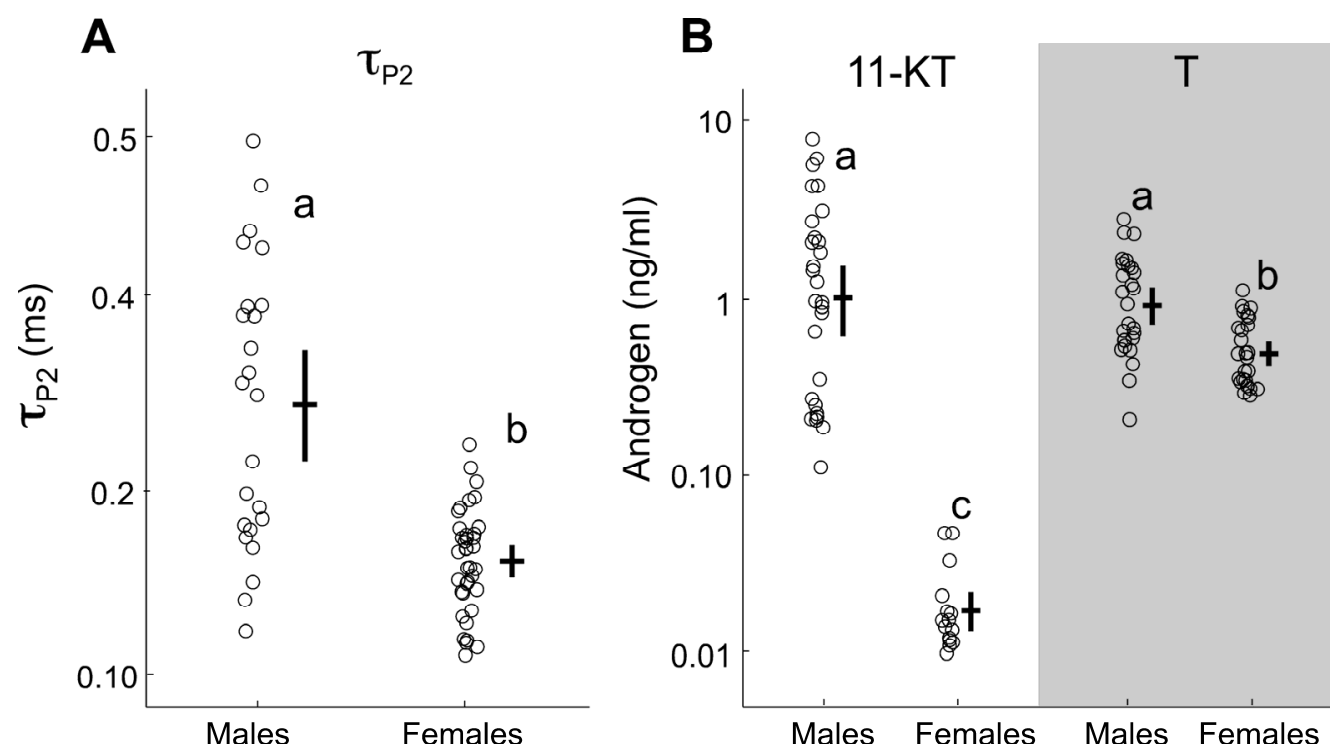

Figure 3.3. Androgen levels and EOD $t_{\mathrm{P} 2}$ parameter in males and females plotted on a log scale. (A) Males have longer duration of the EOD's second phase as estimated by the 
$t_{P 2}$ parameter. (B) Androgen levels in males and females. Horizontal lines indicate group means and vertical lines indicate standard error of log-transformed data. Lower case letters indicate significant differences between groups. Males had greater levels of $\mathrm{T}$ and 11-KT than females. Males had statistically indistinguishable levels of 11-KT and T, while females had significantly higher levels of T than 11-KT.

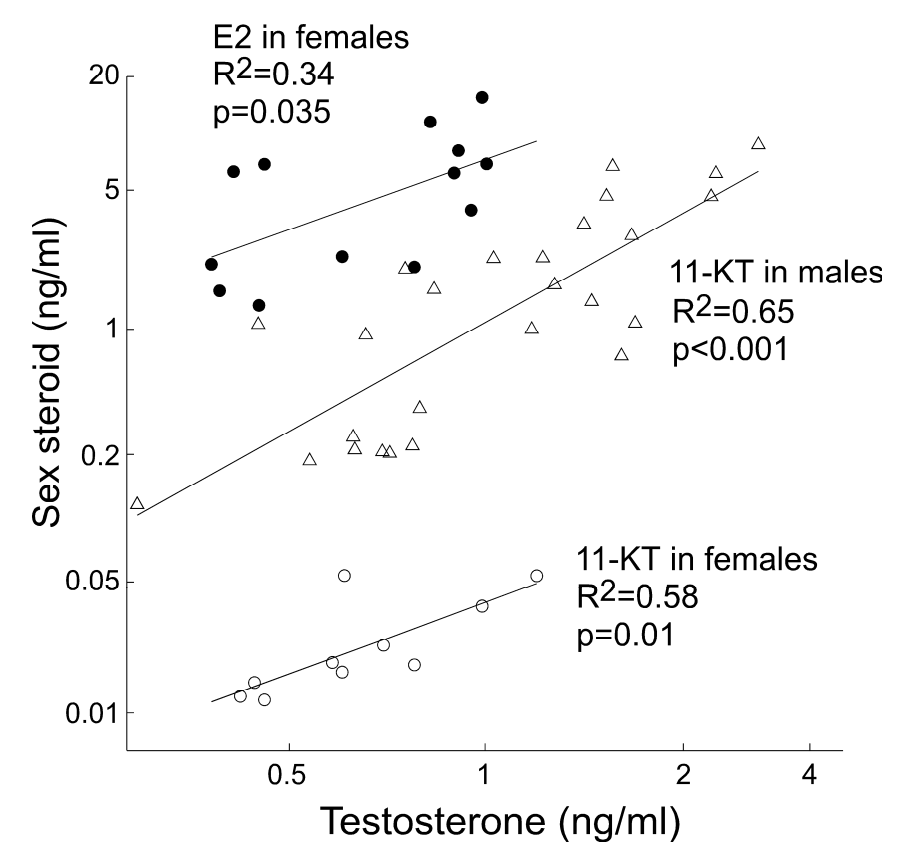

Figure 3.4. Testosterone levels are related to 11-ketotestosterone levels in males and females and estradiol in females, here plotted on a log scale. However, males have more 11-KT per amount of $\mathrm{T}$ than females, while females have high levels of E2 per amount of $\mathrm{T}$. 


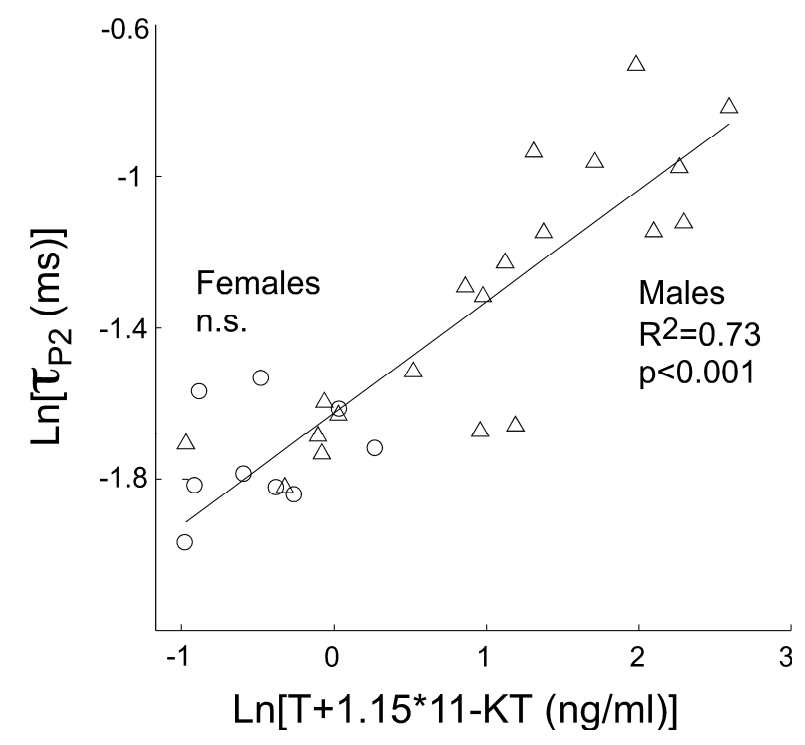

Figure 3.5. First order relationship between androgen levels and the EOD's $t_{p 2}$ parameter. Considering each androgen by itself ignores the important fact that both androgens act on the EOD waveform, so we constructed a model to account for both androgens simultaneously. The weighted sum of circulating androgens is strongly related to EOD $t_{\mathrm{P} 2}$ in males (regression line) but not in females. The difference in the predictability of both androgens for the parameter $t_{\mathrm{P} 2}$ may depend on the relative potency of each androgen in each sex, and on sex differences in circulating levels of androgens (see Discussion). 

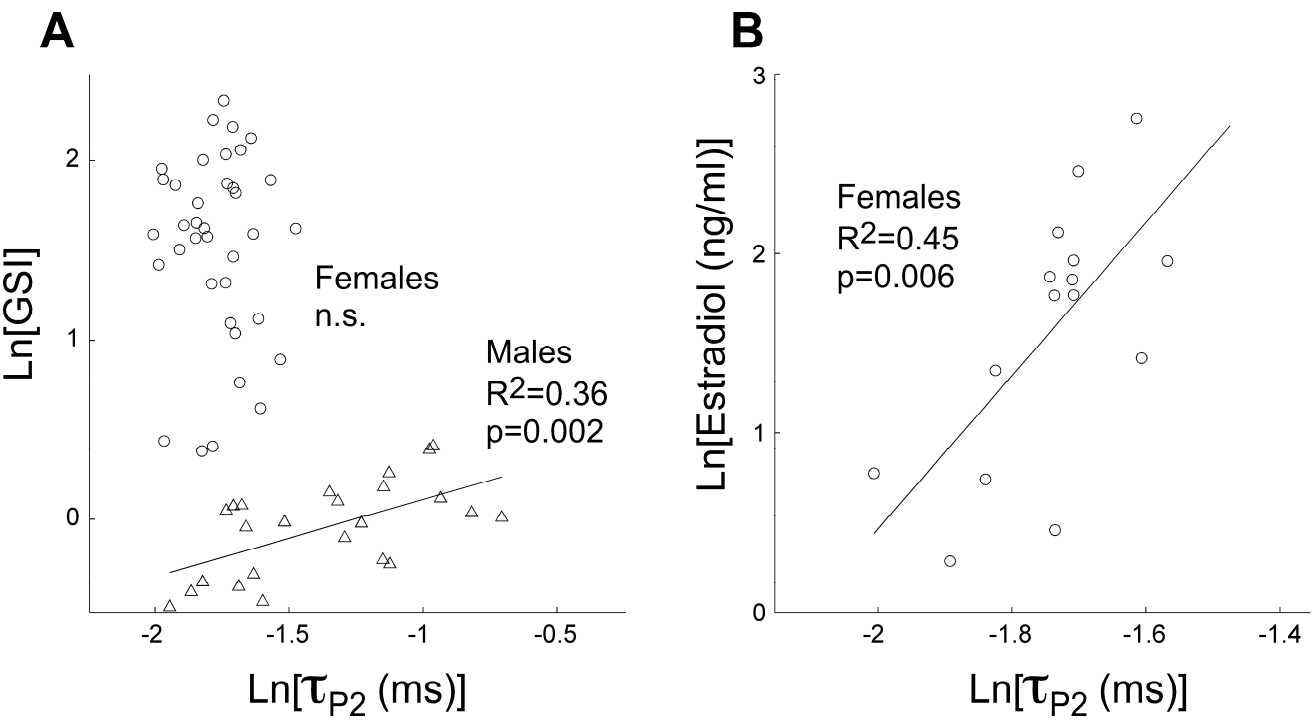

Figure 3.6. The EOD can serve as an indicator of other traits via indirect second order relationships. (A) A receiver could use EOD t $t_{\mathrm{P} 2}$ to predict the gonad size (estimated as GSI) of males but not of females. (B) Similarly, a receiver could use the EOD's $2^{\text {nd }}$ phase elongation parameter, $t_{\mathrm{P} 2}$, to predict circulating levels of estradiol in females. 

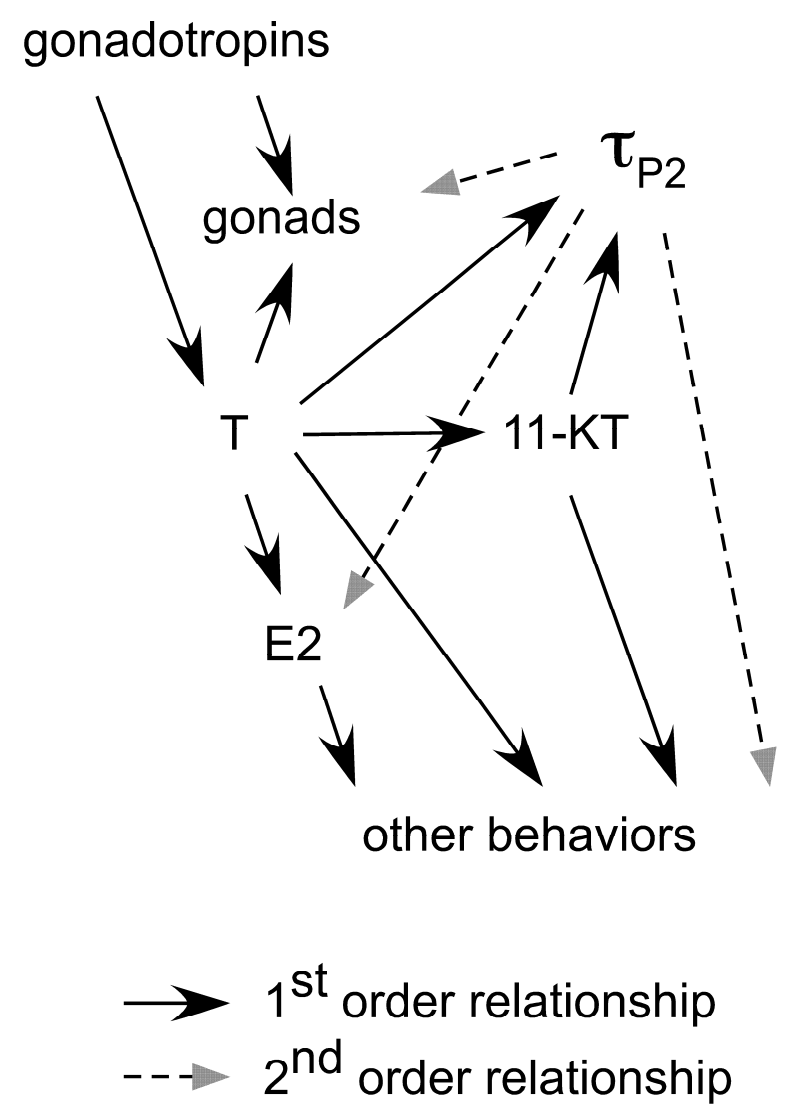

Figure 3.7. In $B$. gauderio the EOD duration parameter $t_{\mathrm{P} 2}$ reliably indicates androgen levels, a relationship that arises from direct action of androgens on EOD $t_{\mathrm{P} 2}$. Through second order relationships, EOD t $t_{\mathrm{P} 2}$ can be used to estimate other, less obvious traits that are also causally related to androgen levels, such as E2 levels, gonad size, and gonad maturation. Both androgens, $\mathrm{T}$ and 11-KT, can serve as intermediaries of these higher order relationships. 


\section{Supplementary table}

\begin{tabular}{|l|l|c|}
\hline \multicolumn{1}{|c|}{ x vs. $\mathbf{y}$} & \multicolumn{1}{c|}{ Males } & Females \\
\hline T vs. EOD $\tau_{\mathrm{P} 2}$ & $\ln y=0.379 \ln x-1.37$ & $\ln y=0.126 \ln x-1.67$ \\
\hline $11-\mathrm{KT}$ vs. EOD $\tau_{\mathrm{P} 2}$ & $\ln y=0.234 \ln x-1.40$ & $\ln y=0.147 \ln x-1.19$ \\
\hline T+1.15*11-KT vs. EOD $\tau_{\mathrm{P} 2}$ & $\ln y=0.294 \ln x-1.63$ & n.s. \\
\hline T vs. 11-KT & $\ln y=1.56 \ln x+0.08$ & $\ln y=1.02 \ln x-3.24$ \\
\hline EOD $\tau_{\mathrm{P} 2}$ vs. GSI & $\ln y=0.431 \ln x+0.543$ & $\mathrm{n} . \mathrm{s.}$ \\
\hline T vs. GSI & $\ln y=0.123 \ln x-0.32$ & $\mathrm{n} . \mathrm{s.}$ \\
\hline $11-\mathrm{KT}$ vs. GSI & $\ln y=0.091 \ln x-0.037$ & $\mathrm{ln} y=1.007 \ln x+2.013$ \\
\hline T vs. E2 & $\mathrm{NA}$ & $\ln y=0.104 \ln x-1.91$ \\
\hline EOD $\tau_{\mathrm{P} 2}$ vs. E2 & $\mathrm{NA}$ & \\
\hline
\end{tabular}

Supplementary table 3.1. Equations for the regression analyses of the traits studied. 


\section{CHAPTER 4}

\section{SOCIAL COMPETITION MASCULINIZES THE COMMUNICATION SIGNALS OF FEMALE ELECTRIC FISH}

As published in Behavioral Ecology and Sociobiology

April 2012

Volume 66, Pages 1057-1066

Copyright (C) Springer 


\begin{abstract}
Male intrasexual competition and female choice explain the evolution of male ornaments. Except in sex-role-reversed taxa, female ornaments have been regarded as an epiphenomenon of genetic correlation, with no female-specific function or independently selected basis. Females from species with conventional sex-roles may still experience some degree of female-female competition and male choice that could explain the persistence of female ornaments. We studied the effect of female competition on the expression of a sexually dimorphic communication signal. In the electric fish Brachyhypopomus gauderio, both sexes produce an electric signal pulse for communication and electrolocation. Male electric pulses are longer in duration and greater in amplitude than those of females. As competition increases, males further enhance their signals in response to elevated androgen levels. We explored whether females respond to social competition as males do, by enhancing their communication signals and increasing androgen levels. We measured amplitude and duration of the electric signal pulse, and testosterone levels in female $B$. gauderio in their natural habitat in Uruguay and estimated social competition by calculating population density and adult sex ratio (ASR). In the lab, we manipulated ASR and population density independently to separate these factors and eliminate seasonal confounds. Under both field and lab conditions, signal pulse amplitude increased with population density while pulse duration increased with female bias in ASR. In the field, but not the lab, androgen levels increased when ASR was female-biased. Our findings indicate that the socially-mediated mechanism of signal regulation is shared by the sexes, although whether androgens regulate this signal plasticity in females remains unclear.
\end{abstract}




\section{Introduction}

In species with conventional sex roles, sexual selection acts on males either through male-male competition or via female choice to produce conspicuous ornaments and behaviors (Andersson 1994). Ornaments serve as communication signals allowing conspecific receivers to infer the quality of the signaler (Bradbury and Vehrencamp 1998). Conversely, sexual selection favoring elaborate ornaments in females has been assumed to be weak or absent, and the presence of male-like ornaments in females has been attributed to genetic correlation with positively selected male traits (Ketterson et al. 2005; Mank 2007). Nonetheless, male mate choice of females has been found even in species with conventional sex roles (Amundsen et al. 1997; Wong and Svensson 2009), demonstrating that some degree of sexual selection acts on females regardless of the mating system (Borg et al. 2002; Forsgren et al. 2004; Clutton-Brock 2007). Likewise, sexual selection may also act on females via female-female competition to promote the evolution of elaborate traits and behaviors. Heightened female competition is common in species with reversed sex-roles (Colwell and Oring 1988; Geberzahn et al. 2009;

Geberzahn et al. 2010), and in species where females compete for direct benefits offered by a male (Yasukawa and Searcy 1982; Sandell and Smith 1997; Langmore 1998; Sandell 1998; Langmore et al. 2002). Less well documented is whether female-female competition has a similar effect on signaling behavior and androgen levels on females of species with conventional sex roles, lacking male parental care or similar direct benefits offered by males to females. 
The social environment is a key factor in determining the intensity of competition and, therefore, the strength of sexual selection acting on a particular sex. For instance when the availability of one sex is limited, the intensity of competition among individuals of the opposite sex will increase while individuals of the limited sex will have greater opportunity to exercise mate choice (Emlen and Oring 1977; Kvarnemo et al. 1995; Borg et al. 2006; Clutton-Brock 2007). The operational sex ratio (OSR), or the ratio of breeding females to breeding males at the site and time of mating, serves as an indicator of the intensity of sexual selection (Emlen and Oring 1977). OSR depends on two factors 1) the adult sex-ratio (ASR), and 2) the potential reproductive rate (PRR) of each sex, which is the maximum number of offspring a given sex can produce per unit of time (Clutton-Brock and Parker 1992). Since PRR depends on the time and energy each sex allocated to their progeny, PRR is set by life-history of the species and the particular sex. On the other hand, OSR is intrinsically correlated to ASR (Clutton-Brock and Parker 1992). Changes in population density can also affect the strength of selection independently of sex-ratio (Kokko and Rankin 2006). For instance, increased encounter rates between same-sex individuals increases competition and the possibility of an individual becoming dominant over a larger number of animals, or the possibility of mate monopolization. Likewise, mate encounter rate may affect mate-choice, or act as a switch point between alternative mating strategies (Kokko and Rankin 2006).

In response to competition, male vertebrates frequently elevate androgen levels and aggressiveness (Wingfield et al. 1990). Androgens, in turn, commonly increase the intensity of sexually selected signals and promote aggression (Moore et al. 2005; Oliveira 
et al. 2005; Ball et al. 2008; Bass 2008; Godwin 2010). The association between androgen levels, aggressive behavior, and signaling effort in social competition is very clear for male vertebrates (Hirschenhauser et al. 2003; Ball and Balthazart 2004; Hirschenhauser and Oliveira 2006). Conversely, socially-mediated changes in behavior and androgen levels in females has received little attention. Nonetheless, females may have significant circulating androgen levels, even in species where the male is the dominant sex (Ketterson et al. 2005; Mank 2007). Whether female aggression is regulated by androgens or other hormones corresponds to differences in breeding systems and the degree of sexual dimorphism (Wingfield et al. 1999a; Wingfield et al. 1999b; Ketterson et al. 2005). Studies in birds have suggested that androgens may play a greater role in monogamous species than in polygamous species, as well as in monomorphic species compared to sexually dimorphic ones (Wingfield et al. 1999b; Ketterson et al. 2005). However, the relationship between $\mathrm{T}$ levels and degree of sexual dimorphism is less clear for polygamous species (Wingfield et al. 1999b). Here we studied whether female-female competition promotes changes in behavior and androgen levels in females from a sexually dimorphic, polygynous species with conventional sex roles.

The electric fish Brachyhypopomus gauderio (Giora \& Malabarba 2009), sister species of $B$. pinnicaudatus, produces a biphasic and sexually dimorphic electric organ discharge (EOD, Fig. 1) for communication and navigation (Hopkins et al. 1990). Males maintain exclusive home ranges and females move among males consistent with an exploded lek polygamous mating system (Miranda et al. 2008). Females have a fractional spawning event about once a week during the breeding season but can spawn several 
times during a spawning night (PKS, unpublished). Males produce EODs of greater amplitude and second phase duration than females (Franchina and Stoddard 1998). At night when active, males and females increase the amplitude and second phase duration of the EOD (Franchina and Stoddard 1998; Stoddard et al. 2007). Under male-male competition, males increase in circulating levels of T and 11-KT (Salazar and Stoddard 2009) and further increase EOD amplitude and second phase duration (Franchina et al. 2001; Salazar and Stoddard 2009). Treating males with T or 11-KT mimics the effects of long-term social interaction on the duration of EOD (Silva et al. 1999; Pouso et al. 2010; Goldina et al. 2011), while 11-KT treatment slightly increases EOD amplitude (Goldina et al. 2011). The duration of the female EOD also increases with androgen administration (Allee et al. 2009), as shown in other pulse-type electric fish with sexually dimorphic second phase extension (Hagedorn and Carr 1985; Bass and Volman 1987). Moreover, EOD duration in both sexes is correlated with androgen levels (Gavassa et al. 2011). Therefore, androgens regulate EOD duration in both sexes of B. gauderio.

We studied whether females respond to social competition as males do, by increasing EOD amplitude and duration along with androgen levels. We investigated the relationship between the intensity of competition, EOD duration, EOD amplitude, and androgen levels in females in an observational study in the field followed by an experimental study in the lab in order to control for any confounding effects observed in the field. 


\section{Materials and Methods}

\section{Field study}

We recorded the EODs of B. gauderio from Laguna Lavalle $\left(32^{\circ} 01.259^{\prime} \mathrm{S}, 055^{\circ}\right.$

22.498'W), Department of Tacuarembó, Uruguay. We sampled five times during the breeding season: October 15-16, 27-28, November 16-18, December 04, 12 and 13, 2009. Fish were located using an audio amplifier to convert their electric signals into sound, netted rapidly, and taken into the EOD recording setup. Only fish with intact tails were retained for analysis (Hopkins et al. 1990). We estimated population abundance by recording the time a skilled fishing team took to capture 30 fish, and defined population density in terms of fish captured per hour. Fish were euthanized by immersion in a eugenol solution $\left(8 \mathrm{mg}^{-1}\right)$ and fixed in $10 \%$ formalin. After $5+$ days of fixation, we determined sex by gonadal inspection. Females with vitelogenic oocytes and males with gonadosomatic index above $0.6 \%$ were considered reproductively active and were used to estimate ASR. We could not estimate OSR directly since OSR depends on both ASR and the PRR of each sex. However, the PRR of B. gauderio has not been determined. Therefore, we used ASR as a proxy for OSR. Anecdotal evidence indicates that males have a higher PRR than females, with males being capable of spawning every night and females spawning every 3-8 nights (PKS, unpublished), as expected for a species with conventional sex roles (Clutton-Brock and Parker 1992). Thus, true OSR will be more male-biased than inferred from ASR alone. Detailed sampling methods are described by Gavassa et al. (2011). 


\section{$\underline{\text { ASR experiment }}$}

We carried out a parallel lab experiment using sexually mature B. gauderio from our eighteenth generation captive-reared colony, from May to October 2010 in Miami, Florida, USA. We manipulated ASR to match the range of values observed in the field, one female per male or four females per male. Seven males and 13 females were held with an opposite sex companion for a week prior to study, then placed in a 450L pool outdoors for another week in groups of either five males and five females (ASR 1:1) or two males and eight females (ASR 1:4). After the week of social treatment, we netted up all the fish and recorded their EODs. We took a blood sample from the first two females captured from each pool. Each ASR treatment was replicated six times, each time using randomly selected fish from our colony, for a total of 30 females in the unity sex ratio treatment and 48 females in the $4: 1$ treatment.

\section{$\underline{\text { Population abundance experiment }}$}

We randomly selected 18 females and 18 males $B$. gauderio from our captive-reared colony. We tagged the fish with individual alphanumeric elastomer tags and placed them at random in outdoor 450L pools of 2, 6, or 12 fish, keeping sex ratio at unity. After a week of social treatment, we recorded their EODs. In between social treatments, fish remained with an opposite sex companion for a week. Female fish went through all treatments in random order, except for one female who died and was replaced with another female from our colony. Treatments were temporally interspersed; we run the experiment in three rounds, in each round we had six fish per treatment. We did not take blood samples from females in this experiment, since blood sampling would impair the 
females from going through all the treatments. Detailed methods are described by Gavassa et al. (2012).

\section{EOD recordings}

Field EOD recordings took place inside the lagoon in a submerged plastic mesh cage $(100 \times 50 \times 50 \mathrm{~cm})$ with recording electrodes located at either end, $100 \mathrm{~cm}$ apart. A mesh tube held the fish lengthwise, equidistant from the recording electrodes and $25 \mathrm{~cm}$ below the water surface. A ground electrode was located perpendicular to the fish. Water conductivity was $38-57 \mu \mathrm{S} \mathrm{cm}^{-1}$ and water temperature was $19-28^{\circ} \mathrm{C}$ throughout the field study. In the lab, we recorded the EODs inside a glass aquarium (120 x $40 \times 40 \mathrm{~cm})$, in water adjusted to $100 \pm 6 \mu \mathrm{S} / \mathrm{cm}$ and $25.8 \pm 1.3^{\circ} \mathrm{C}$.

In the field and in the lab, EODs were differentially amplified 100x (WPI, Inc. DAM-50, AC-coupled, highpass filter corner $0.1 \mathrm{~Hz}$, lowpass filter corner $10 \mathrm{KHz}$ ). Signals were digitized by an RM1 mobile processor (Tucker Davis Technologies, Alachua, FL) at a sampling rate of $48.8 \mathrm{kHz}$, and stored and analyzed on a portable computer using custom software developed in MATLAB. We analyzed the EODs of fish with intact caudal filaments, determining peak-to-peak amplitude $(\mathrm{mV})$ and second phase duration as the parameter $\tau_{\mathrm{P} 2}$, the time constant of repolarisation of the second phase (Fig. 1; Stoddard et al. 2003).

\section{Blood collection and hormone assays}

After recording their EODs, we anesthetized the fish by immersion in eugenol solution $\left(1.2 \mathrm{mg} \mathrm{l}^{-1}\right)$ for about a minute. Within five minutes of capturing the fish, we drew blood from the ventral vertebral sinus into a syringe containing $5 \mu 1$ 10\% EDTA, then 
centrifuged the blood for $15 \mathrm{~min}$ at $6000 \mathrm{rpm}$ at $4^{\circ} \mathrm{C}$, transferring the plasma to a polypropylene tube to be stored at $-80^{\circ} \mathrm{C}$ until analysis.

We measured total levels of circulating sex steroids following a validated protocol of extraction and quantification for this species (Salazar and Stoddard 2009). In the field we measured T, 11-KT and E2. However, since we were short on funds, and all these steroid hormones are highly correlated in this species and females have very little 11-KT (Gavassa et al. 2011), we only measured T in the lab population. Detailed hormone assay protocols are described in Gavassa et al. (2011).

\section{$\underline{\text { Data analysis }}$}

We normalized all the variables with a natural log transform, verified with Shapiro-Wilk test. For the field observations, we performed Pearson correlations between EOD parameters (amplitude and duration), hormone levels (T, 11-KT, and E2) with population density and ASR. For the population density lab manipulations we used repeated measures analysis of variance (ANOVA) to test whether population density had a direct effect on EOD parameters. For the ASR manipulation, we used t-test to examine the difference between the two ASR treatments in EOD parameters and testosterone levels. We used MATLAB Statistical Toolbox for statistical analysis.

\section{Results}

ASR in the field varied from 4.0 to 1.0 females per male (4.0, 3.7, 0.6, 0.7 and 1.5; probability of unity: $P=0.05, P=0.03, P=0.3, P=0.3$, and $P=0.3$ respectively; 
collection dates: October 15-16 and 27-28, November 16-18, December 4 and 12-13, 2009 respectively). Population density in the field varied from 4.5 to 10 fish captured per hour. Season was confounded with other variables in our field observations. Seasonal progression correlated with ASR and water temperature, but not with population density nor water conductivity (ASR: $r=-0.93, P=0.02$; water temperature: $r=0.82, P=0.01$; population density: $r=0.25, P=0.68$; water conductivity: $r=0.41, P=0.31$ ).

The strong correlation between time into the breeding season and ASR makes multiple linear regression useless to disentangle which one of those two variables has a stronger effect on the EOD. Thus, we performed individual linear regressions using time into the breeding season and ASR separately. EOD amplitude was positively correlated with population density $(r=0.57, P<0.001$; Fig. $2 \mathrm{a}$, Table 1$)$, and time into the breeding season $(r=0.37, P=0.001$; Fig. $2 \mathrm{c})$, but negatively correlated with female-bias in ASR $(r=-0.34, P=0.004$; Fig. $2 \mathrm{~b})$. On the other hand, EOD duration $\left(\tau_{\mathrm{P} 2}\right)$ did not correspond to population density ( $r=-0.03, P=0.78$; Fig. $2 \mathrm{~d}$ ), though we found a strong positive correlation between $\tau_{\mathrm{P} 2}$ in the field and female-bias in $\operatorname{ASR}(r=0.61, P<0.001$; Fig. $2 \mathrm{e})$. However, $\tau_{\mathrm{P} 2}$ also shows a strong seasonal effect, decreasing with time into the breeding season $(r=-0.70, P<0.001$; Fig. $2 \mathrm{f})$.

In the field, changes in sex steroid levels parallel those found in the $\tau_{\mathrm{P} 2}$ parameter of female EODs. Female-bias in ASR corresponded to elevated T, 11-KT, and E2 levels in females (T: $r=0.53, P<0.001 ; 11-\mathrm{KT}: r=0.77, P<0.001$; E2: $r=0.56, P=0.003$; Fig. 3 a \& c). Here again, a negative seasonal trend is evident in T, 11-KT, and E2, all of which declined in serum concentration as the breeding season progressed (T: $r=-0.53, P$ 
$<0.001$; 11-KT: $r=-0.74, P<0.001$; E2: $r=-0.63, P<0.001$; Fig. 3 b\&d). Because of seasonal trends, we consider manipulative lab tests critical for testing causality of effects seen in the field.

As in the field, EOD amplitude in the lab study increased with population density (Repeated measures ANOVA, $F_{1,16}=12.89, P=0.002, n=17$; Fig. 4a). However in contrast to the field observations, our lab experiment yielded no effect of ASR on female EOD amplitude (t-test: $t_{\text {stat }}=0.59$, d.f. $=74, P=0.55$; Fig. $4 \mathrm{~b}$, Table 1).

Unlike the field, population density in the lab did correlate with EOD duration $\left(\tau_{\mathrm{P} 2}\right)$ (repeated measures ANOVA, $F_{1,16}=58.01, P<0.001, n=17$; Fig. $4 \mathrm{c}$ ). In the lab as in the field, $\tau_{\mathrm{P} 2}$ was higher in the treatment with a female-biased ASR (t-test, $t_{\text {stat }}=-2.43$, d.f. $=74, P=0.017$; Fig. $4 \mathrm{~d}$ ). On the other hand, the lab manipulations of ASR produced no difference in females' $\mathrm{T}$ levels (t-test, $t_{\text {stat }}=1.01$, d.f. $=19, P=0.32$; Fig. 5). We did not measure $\mathrm{T}$ levels in females at different fish density treatments.

\section{Discussion}

We found that females respond to social competition by increasing the amplitude of their EODs as population density increases, and they respond to intrasexual competition by elongating their EODs if the adult sex-ratio becomes female-biased. In the field, androgen levels were correlated with the intensity of female intrasexual competition, although this relationship was confounded by other variables and no such correlation was found in the lab. In response to social competition, female $B$. gauderio enhance their 
electric signal waveforms the same way as males, just to a lesser extent. Females did not express the strong effect of social competition on androgen levels seen in males, which may account for the difference in degree of signal enhancement displayed by the two sexes in response to same-sex competition.

Across our field and lab studies, EOD amplitude appeared to be driven by population density, whereas EOD second phase duration $\left(\tau_{\mathrm{P} 2}\right)$ responded strongly to ASR. EOD amplitude enhancements may signal resource holding potential under social competition (Salazar 2009; Silva et al. 2010; Gavassa et al. 2012) or may improve electrolocation by overcoming the increase in background noise that results from greater population density. Both field and lab studies provided strong evidence that females increase $\tau_{\mathrm{P} 2}$ with female intrasexual competition. Thus, duration of the EOD's second phase may serve as a signal during intrasexual competition while EOD amplitude may function as a signal for competition between and within sexes. Interestingly, changes in female EOD waveform parallel those found in males (Salazar and Stoddard 2009; Gavassa et al. 2012). Our results show that females are capable of responding to social competition just as males do.

Effects that were unique to our observational field studies or our experimental lab studies might have been real, the result of confounds, or our inability to fully replicate field conditions in the lab. For instance, only in the field did EOD amplitude decrease as ASR became female-biased. This result could be explained by a seasonal effect on EOD amplitude that is confounded with ASR in the field (Fig. 2). In the lab, but not the field, females increased EOD pulse duration $\left(\tau_{\mathrm{P} 2}\right)$ as population density increased. In the lab, 
we created a range of population densities three times greater than we measured in the field, thus the more extreme change in population density in the lab could explain why females increased $\tau_{\mathrm{P} 2}$ with population density only there. Alternatively, the underlying seasonal variation may have prevented us from finding a relationship between population density and $\tau_{\mathrm{P} 2}$ in the field.

Although testosterone levels did not increase with competition in our experiment, a prior lab study found that females in the lab housed in outdoor breeding pools under high competition ( 6 females with either 2 or 6 males per pool) had circulating T levels that surpassed those of males (Salazar and Stoddard 2009). Perhaps the effect of social competition on androgen levels is sensitive to time in the breeding season. Nonetheless, we did find an increase in EOD duration, which is regulated by androgens (Silva et al. 2002; Allee et al. 2009; Pouso et al. 2010; Goldina et al. 2011). It is possible that the change in signal duration we found in the lab resulted from a small change in androgen levels that we failed to detect. Androgens mediate long-term changes in EOD duration (Silva et al. 2002; Allee et al. 2009; Goldina and Stoddard 2009), while peptide hormones mediate short-term changes (Markham and Stoddard 2005; Stoddard et al. 2006; Markham et al. 2009). Furthermore, androgens greatly potentiate the effect of melanocortin administration on EOD duration (Allee et al. 2009; Goldina et al. 2011). Therefore, a small increase in androgens could result in a much greater increase in EOD duration through synergy with melanocortin action.

Given their life-history (exploded lek polygyny, larger male signal structures, no parental care; Franchina and Stoddard 1998; Miranda et al. 2008), one might not predict 
female $B$. gauderio would respond to intrasexual competition. Female B. gauderio do not defend territories (Miranda et al. 2008) nor compete for direct male benefits or parental care; however, females may compete for access to high quality males, particularly when males are scarce. In the Japanese medaka (Oryzias latipes) (Clark and Grant 2010) and the sand goby (Pomatoschistus minutus) (Kvarnemo et al. 1995), females compete for access to males when OSR is female-biased, while males compete with other males when OSR is male-biased. In the electric fish B. gauderio, female aggression may prevent other females from interrupting their mating bouts, as suggested for other species (Karvonen et al. 2000). At the beginning of the breeding season the relative scarcity of males may result from males taking longer than females to mature. In order to breed, females only have to develop a few oocytes at a time (Quintana et al. 2004) while males have to grow to a certain size that is attractive to females (Curtis and Stoddard 2003). However, breeding early is often very advantageous, thus females might be under great pressure to find the best mate to fertilize their few eggs at the beginning of the season and so intrasexual competition may be critical to their fitness.

Although we didn't test directly whether EOD enhancement provides females with a mating advantage as expected from a sexually-selected trait, we are confident that signal enhancements are not the result of natural selection. We see compelling evidence of natural selection constraining the evolution of conspicuous signals (Stoddard 1999; Stoddard 2002; Salazar and Stoddard 2008). The EOD is expensive in predation terms, elongating the duration of the EOD's second phase makes the signal more conspicuous to predators (Hanika and Kramer 1999; Stoddard 1999; Hanika and Kramer 2000). Electric 
signals are also energetically expensive, their cost increasing with the amplitude and the duration of the signal (Salazar and Stoddard 2008). Female signals are less expensive and less conspicuous than male signals (Stoddard 1999; Salazar and Stoddard 2008). When competition is low, males reduce their signaling effort to reduce the energetic and predation cost of signaling. However, once competition resumes, males regain the handicap costs of signaling (Gavassa et al. 2012). Enhancing the amplitude of the signal increases the honesty of the information conveyed by the signal. Body length is tightly related to EOD amplitude and this relationship improves as EOD amplitude increases during competition as males signal the closest to their physiological limit (Gavassa et al. 2012). Body size information is key for mate choice and male-male aggression, longer males are preferred by females (Curtis and Stoddard 2003) and are more likely to win an agonistic encounter (Salazar 2009; Silva et al. 2010). On the other hand, signal duration in males and females conveys information about the reproductive state of the signaler, and possibly its aggressive intent (Gavassa et al. 2011). Thus, elongating the signals may serve to attract mates and it may also warn competitors about the aggressiveness of the signaler.

Signal plasticity allows both sexes to adjust their signaling effort and subsequent signaling cost to the intensity of competition. In both males and females, intrasexual competition promotes the elongation of the duration of the EOD's second phase, while intersexual competition promotes the increase in EOD amplitude. The similarity in the response to competition in males and females suggests either 1) both sexes face parallel selective forces, albeit with different strength, or 2) female behavior may still be the 
result of genetic correlations with sexually selected male traits, despite strong natural selection to make female signals less conspicuous and less expensive (Stoddard 1999; Stoddard and Salazar 2011). Signal amplitude and duration is greater in males than in females (Franchina and Stoddard 1998; Stoddard et al. 2007; Salazar and Stoddard 2008) indicating that overall sexual selection is stronger in males of this species than in females. If both sexes are subjects of sexual selection, the temporal changes in the population structure seem to be responsible for the maintenance of some degree of sexual selection in females. However, future studies are needed to test whether signal enhancements provide females with a competitive advantage, as would be expected if female signals are indeed under direct sexual selection.

The strength of sexual selection on a given sex depends on the OSR. We could not measure OSR directly since we do not have an accurate estimate of the PRR for each sex of B. gauderio. Nonetheless, the PRR of females is lower than that of males (see methods) so that a unity ASR is actually a male-biased OSR. If the difference in the PRR between the sexes is high enough, the OSR may still be male-biased even when the ASR is female-biased. Throughout the time we sampled we found either a unity ratio or a female-based ratio. Therefore, we do see an increase in female competition as ASR becomes female-biased, but the OSR may still be male-biased resulting in stronger sexual selection in males than in females.

We show that the social environment can have great effects in female behavior through same-sex competition. Interestingly, the presence of female-female competition can neither be explained by sex role reversal nor by competition for male direct benefits. 
We conclude that female intrasexual competition may be more common than expected from theory on mating systems. Particularly, our study highlights the importance of temporal variation in population structure affecting the degree of sexual selection experienced by either sex.

\section{Acknowledgments}

We thank R. Perrone, L. Zubizarreta, G. Batista, T. de los Campos, D. Colacce, RV. Gavassa, I. Becerra, P. Pouso, and J. Campbell for assistance in the field. We thank O. Macadar, Ministerio de Cultura y Educación, Uruguay, and Facultad de Ciencias, Universidad de la República, Montevideo, Uruguay, for logistic assistance in the field. We thank L. Kim and F. Noriega for use of the plate reader, and A. Goldina and V. Salazar for assistance with immunoassays. We thank E. Ketterson and two anonymous reviewers for helpful comments on the manuscript. This work was supported by Tinker Field Research Grants (LACC at FIU) to S.G., University Graduate School's Dissertation Evidence Acquisition Fellowship and Dissertation Year Fellowship to S.G., Judith Evans Scholarship for travel to S.G., and Animal Behavior Society award to S.G. and NSF grant IOS 0956603 to P.K.S. This paper is contribution \# 226 to the FIU Tropical Biology Program.

Ethical Standards Collections and experimental procedures were performed under the guidelines and approval of the Comisión Honoraria de Experimentación Animal, Universidad de la República, Montevideo, Uruguay, and by the Institutional Animal Care and Use Committee of the Florida International University, Miami, FL (protocols 08-027 and 10-020). 


\section{References}

Allee SJ, Markham MR, Stoddard PK (2009) Androgens enhance plasticity of an electric communication signal in female knifefish, Brachyhypopomus pinnicaudatus. Horm Behav 56:264-273

Amundsen T, Forsgren E, Hansen LTT (1997) On the function of female ornaments: male bluethroats prefer colourful females. Proc R Soc Lond B 264:1579-1586

Andersson M (1994) Sexual Selection. Princeton University Press, New Jersey

Ball GF, Balthazart J (2004) Hormonal regulation of brain circuits mediating male sexual behavior in birds. Physiol Behav 83:329-346

Ball GF, Tlemçani O, Balthazart J (2008) Individual variation and the endocrine regulation of behaviour and physiology in birds: a cellular/molecular perspective. Philos Trans R Soc Lond B 363:1699-1710

Bass AH (2008) Steroid-dependent plasticity of vocal motor systems: Novel insights from teleost fish. Brain Res Rev 57:299-308

Bass AH, Volman SF (1987) From behavior to membranes: testosterone-induced changes in action potential duration in electric organs. Proc Natl Acad Sci USA 84:92959298

Borg AA, Forsgren E, Amundsen T (2006) Seasonal change in female choice for male size in the two-spotted goby. Anim Behav 72:763-771

Borg AA, Forsgren E, Magnhagen C (2002) Plastic sex-roles in the common goby - the effect of nest availability. Oikos 98:105-115

Bradbury JW, Vehrencamp SL (1998) Principles of animal communication. Sinauer Associates, Sunderland, MA

Clark L, Grant JWA (2010) Intrasexual competition and courtship in female and male Japanese medaka, Oryzias latipes: effects of operational sex ratio and density. Anim Behav 80:707-712

Clutton-Brock T (2007) Sexual selection in males and females. Science 318:1882-5 
Clutton-Brock TH, Parker GA (1992) Potential reproductive rates and the operation of Sexual Selection. The Quarterly Review of Biology 67:437-456

Colwell MA, Oring LW (1988) Sex ratios and intrasexual competition for mates in a sexrole reversed shorebird, Wilson's phalarope (Phalaropus tricolor). Behav Ecol Sociobiol 22:165-173

Curtis CC, Stoddard PK (2003) Mate preference in female electric fish, Brachyhypopomus pinnicaudatus. Anim Behav 66:329-336

Emlen ST, Oring LW (1977) Ecology, sexual selection, and the evolution of mating systems. Science 197:215-23

Forsgren E, Amundsen T, Borg AA, Bjelvenmark J (2004) Unusually dynamic sex roles in a fish. Nature 429:551-554

Franchina CR, Salazar VL, Volmar CH, Stoddard PK (2001) Plasticity of the electric organ discharge waveform of male Brachyhypopomus pinnicaudatus II. Social effects. J Comp Physiol A 187:45-52

Franchina CR, Stoddard PK (1998) Plasticity of the electric organ discharge waveform of the electric fish Brachyhypopomus pinnicaudatus. I. Quantification of day-night changes. J Comp Physiol A 183:759 - 768

Gavassa S, Silva A, Gonzalez E, Stoddard PK (2012) Signal modulation as a mechanism for handicap disposal. Anim Behav 83:935-944

Gavassa S, Silva AC, Stoddard PK (2011) Tight hormonal phenotypic integration ensures honesty of the electric signal of male and female Brachyhypopomus gauderio. Horm Behav 60:420-426

Geberzahn N, Goymann W, Muck C, ten Cate C (2009) Females alter their song when challenged in a sex-role reversed bird species. Behav Ecol Sociobiol 64:193-204

Geberzahn N, Goymann W, ten Cate C (2010) Threat signaling in female song-evidence from playbacks in a sex-role reversed bird species. Behav Ecol 21:1147-1155

Giora J, Malabarba LR (2009) Brachyhypopomus gauderio, new species, a new example of underestimated species diversity of electric fish in the southern South America (Gymnotiformes: Hypopomidae). Zootaxa 2093:60-68 
Godwin J (2010) Neuroendocrinology of sexual plasticity in teleost fishes. Front Neuroendocrin 31:203-16

Goldina A, Gavassa S, Stoddard PK (2011) Testosterone and 11-ketotestosteone have different regulatory effects on electric communication signals of male Brachyhypopomus gauderio. Horm Behav 60:139-147

Goldina A, Stoddard PK (2009) Testosterone insensitivity is related to sociallity levels in gymnotiform fish. In: The society for behavioral endocrinology, East Lansing, MI

Hagedorn M, Carr C (1985) Single electrocytes produce a sexually dimorphic signal in South American electric fish, Hypopomus occidentalis (Gymnotiformes, Hypopomidae). J Comp Physiol A 156:511-523

Hanika S, Kramer B (1999) Electric organ discharges of mormyrid fish as a possible cue for predatory catfish. Naturwissenschaften $86: 286-288$

Hanika S, Kramer B (2000) Electrosensory prey detection in the African sharptooth catfish, Clarias gariepinus (Clariidae), of a weakly electric mormyrid fish, the bulldog (Marcusenius macrolepidotus). Behav Ecol Sociobiol 48:218-228

Hirschenhauser K, Oliveira RF (2006) Social modulation of androgens in male vertebrates: meta-analyses of the challenge hypothesis. Anim Behav 71:265-277

Hirschenhauser K, Winkler H, Oliveira RF (2003) Comparative analysis of male androgen responsiveness to social environment in birds: the effects of mating system and paternal incubation. Horm Behav 43:508-519

Hopkins CD, Comfort NC, Bastian J, Bass AH (1990) Functional analysis of sexual dimorphism in an electric fish, Hypopomus pinnicaudatus, order Gymnotiformes. Brain Behav Evolut 35:350-67

Karvonen E, Rintamaki PT, Alatalo RV (2000) Female-female aggression and female mate choice on black grouse leks. Anim Behav 59:981-987

Ketterson ED, Nolan V, Sandell M (2005) Testosterone in females: mediator of adaptive traits, constraint on sexual dimorphism, or both? Am Nat 166:S85-S98 
Kokko H, Rankin DJ (2006) Lonely hearts or sex in the city? Density-dependent effects in mating systems. Philos Trans R Soc Lond B 361:319-334

Kvarnemo C, Forsgren E, Magnhagen C (1995) Effects of sex ratio on intra- and intersexual behaviour in sand gobies. Anim Behav 50:1455-1461

Langmore NE (1998) Functions of duet and solo songs of female birds. Trends Ecol Evol $13: 136-140$

Langmore NE, Cockrem JF, Candy EJ (2002) Competition for male reproductive investment elevates testosterone levels in female dunnocks, Prunella modularis. Proc R Soc Lond B 269:2473-2478

Mank JE (2007) The evolution of sexually selected traits and antagonistic androgen expression in actinopterygiian fishes. Am Nat 169:142-149

Markham M, Allee S, Goldina A, Stoddard P (2009) Melanocortins regulate the electric waveforms of gymnotiform electric fish. Horm Behav 55:306-313

Markham MR, Stoddard PK (2005) Adrenocorticotropic hormone enhances the masculinity of an electric communication signal by modulating the waveform and timing of action potentials within individual cells. J Neurosci 25:8746-8754

Miranda M, Silva A, Stoddard PK (2008) Use of space is consistent with exploded lek polygyny in the gymnotiform electric fish Brachyhypopomus pinnicaudatus. Environ Biol Fish 83:379-389

Moore FL, Boyd SK, Kelley DB (2005) Historical perspective: Hormonal regulation of behaviors in amphibians. Horm Behav 48:373-83

Oliveira RF, Ros AF, Goncalves DM (2005) Intra-sexual variation in male reproduction in teleost fish: a comparative approach. Horm Behav 48:430-9

Pouso P, Quintana L, Bolatto C, Silva AC (2010) Brain androgen receptor expression correlates with seasonal changes in the behavior of a weakly electric fish, Brachyhypopomus gauderio. Horm Behav 58:729-36

Quintana L, Silva A, Berois N, Macadar O (2004) Temperature induces gonadal maturation and affects electrophysiological sexual maturity indicators in 
Brachyhypopomus pinnicaudatus from a temperate climate. J Exp Biol 207:18431853

Salazar VK (2009) The effect of male-male competition and its underlying regulatory mechanisms on the electric signal of the gymnotiform fish Brachyhypopomus gauderio. Dissertation, Florida International University

Salazar VL, Stoddard PK (2008) Sex differences in energetic costs explain sexual dimorphism in the circadian rhythm modulation of the electrocommunication signal of the gymnotiform fish Brachyhypopomus pinnicaudatus. J Exp Biol 211:1012-1020

Salazar VL, Stoddard PK (2009) Social competition affect electric signal plasticity and steroid hormone levels in the gymnotiform fish Brachyhypopomus gauderio. Horm Behav 56:399-409

Sandell MI (1998) Female aggression and the maintenance of monogamy: female behaviour predicts male mating status in European starlings. Proc R Soc Lond B 265:1307-1311

Sandell MI, Smith HG (1997) Female aggression in the European starling during the breeding season. Anim Behav 53:13-23

Silva A, Quintana L, Ardanaz JL, Macadar O (2002) Environmental and hormonal influences upon EOD waveform in gymnotiform pulse fish. J Physiology-Paris 96:473-484

Silva A, Quintana L, Galeano M, Errandonea P, Macadar O (1999) Water temperature sensitivity of EOD waveform in Brachyhypopomus pinnicaudatus. J Comp Physiol A 185:187-197

Silva A, Zubizarreta L, Costa G (2010) Interspecific differences in agonistic behavior and its serotonergic modulation. In: International Congress of Neuroethology, Salamanca, Spain

Stoddard PK (1999) Predation enhances complexity in the evolution of electric fish signals. Nature 400:254-256 
Stoddard PK (2002) Electric signals: Predation, sex, and environmental constraints. Advances in the Study of Behavior 31:201-242

Stoddard PK, Markham MR, Salazar VL (2003) Serotonin modulates the electric waveform of the gymnotiform electric fish Brachyhypopomus pinnicaudatus. J Exp Biol 206:1353-1362

Stoddard PK, Markham MR, Salazar VL, Allee S (2007) Circadian rhythms in electric waveform structure and rate in the electric fish Brachyhypopomus pinnicaudatus. Physiol Behav 90:11-20

Stoddard PK, Salazar VL (2011) Energetic cost of communication. J Exp Biol 214:200205

Stoddard PK, Zakon HH, Markham MR, McAnelly L (2006) Regulation and modulation of electric waveforms in gymnotiform electric fish. J Comp Physiol A 192:613 624

Wingfield J, Hegner RE, Dufty Jr AM, Ball G (1990) The "challenge hypothesis": theoretical implications for patterns of testosterone secretion, mating systems, and breeding strategies. Am Nat 136:829-846

Wingfield JC, Jacobs JD, Soma K, Maney DL, Hunt K, Wisti-Peterson D, Meddle S, Ramenofsky M, Sullivan K (1999a) Testosterone, aggression and communication: ecological bases of endocrine phenomena. In: Hauser M, Konishi M (eds) The Design of Animal Communication. M.I.T. Press, Cambridge

Wingfield JC, Jacobs JD, Tramontin AD, Perfito N, Meddle S, Maney DL, Soma K (1999b) Toward an ecological basis of hormone-behavior interactions in reproduction in birds. In: Wallen K, Schneider J (eds) Reproduction in Context. M.I.T. Press, Cambridge, MA

Wong B, Svensson P (2009) Strategic male signalling effort in a desert-dwelling fish. Behav Ecol Sociobiol 63:543-549

Yasukawa K, Searcy WA (1982) Aggression in female red-winged blackbirds - a strategy to ensure male parental investment. Behav Ecol Sociobiol 11:13-17 
Table

\begin{tabular}{cccccc}
\hline & \multicolumn{2}{c}{ Density } & \multicolumn{2}{c}{ ASR } & Season \\
& Field & Lab & Field & Lab & Field \\
\hline $\begin{array}{c}\text { EOD amplitude } \\
\text { EOD duration }\end{array}$ & $\uparrow$ & $\uparrow$ & $\downarrow$ & - & $\uparrow$ \\
$\left(\boldsymbol{\tau}_{\mathrm{P} 2}\right)$ & - & $\uparrow$ & $\uparrow$ & $\uparrow$ & $\downarrow$ \\
Testosterone & - & NA & $\uparrow$ & - & $\downarrow$ \\
\hline
\end{tabular}

Table 4.1. Summary of changes in electric organ discharge (EOD) parameters and testosterone levels with population density, adult sex-ratio (ASR) and season between the field observations and the lab manipulations. Horizontal line indicates non-significant change. Testosterone levels were not assayed (NA) for population density manipulation in the lab 


\section{Figures}

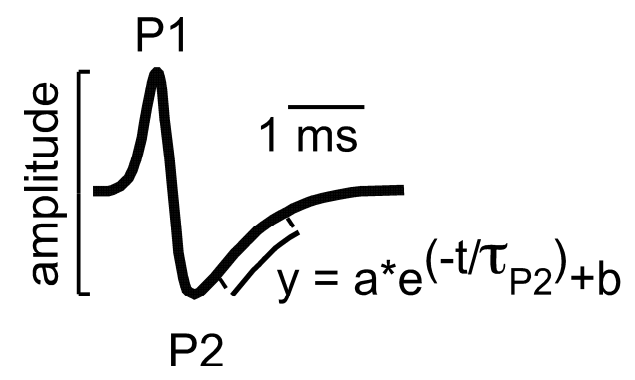

Figure 4.1. The electric organ discharge (EOD) waveform of B. gauderio is composed of a positive phase (P1) and a negative phase (P2). Amplitude was measured peak-to-peak. Duration of the second phase was characterized by second phase duration $\left(\tau_{\mathrm{P} 2}\right)$, the time constant of repolarization 

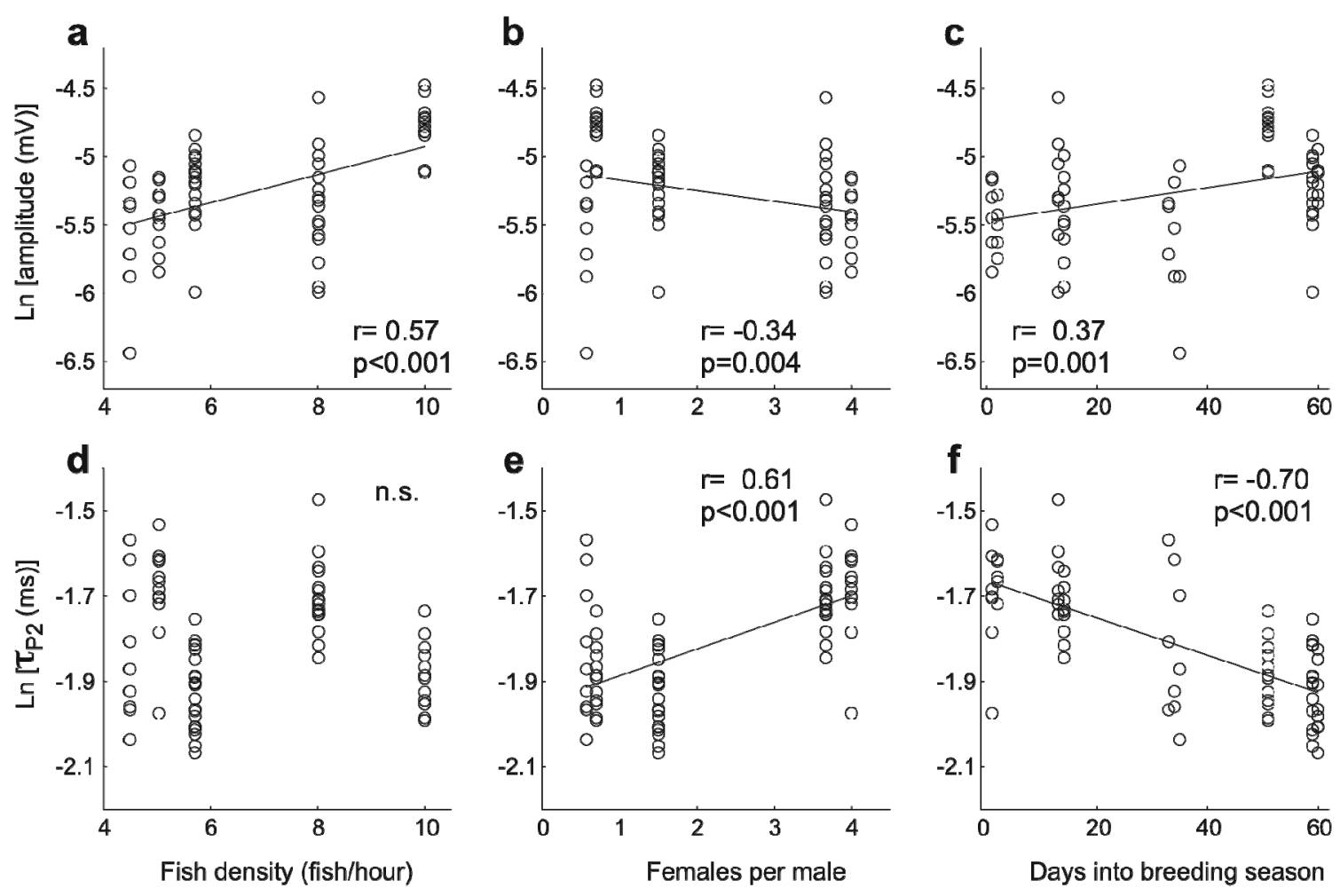

Figure 4.2. Electric organ discharge (EOD) waveform parameters in the field; amplitude and second phase duration $\left(\tau_{\mathrm{P} 2}\right)$, vary with adult sex-ratio (ASR), density, and time into the breeding season. In the field, EOD amplitude was positively correlated with population density (a) and with time into the breeding season (c). b, EOD amplitude was negatively correlated with ASR. d, EOD duration $\left(\tau_{\mathrm{P} 2}\right)$ did not vary with population density in the field. e, As the ASR became more female-biased, $\tau_{\mathrm{P} 2}$ increased. $\mathbf{f}$, Conversely, $\tau_{\mathrm{P} 2}$ decreased with time into the breeding season. Circles depict raw data, correlation lines are shown when the correlation was significant $(P<0.05)$ 

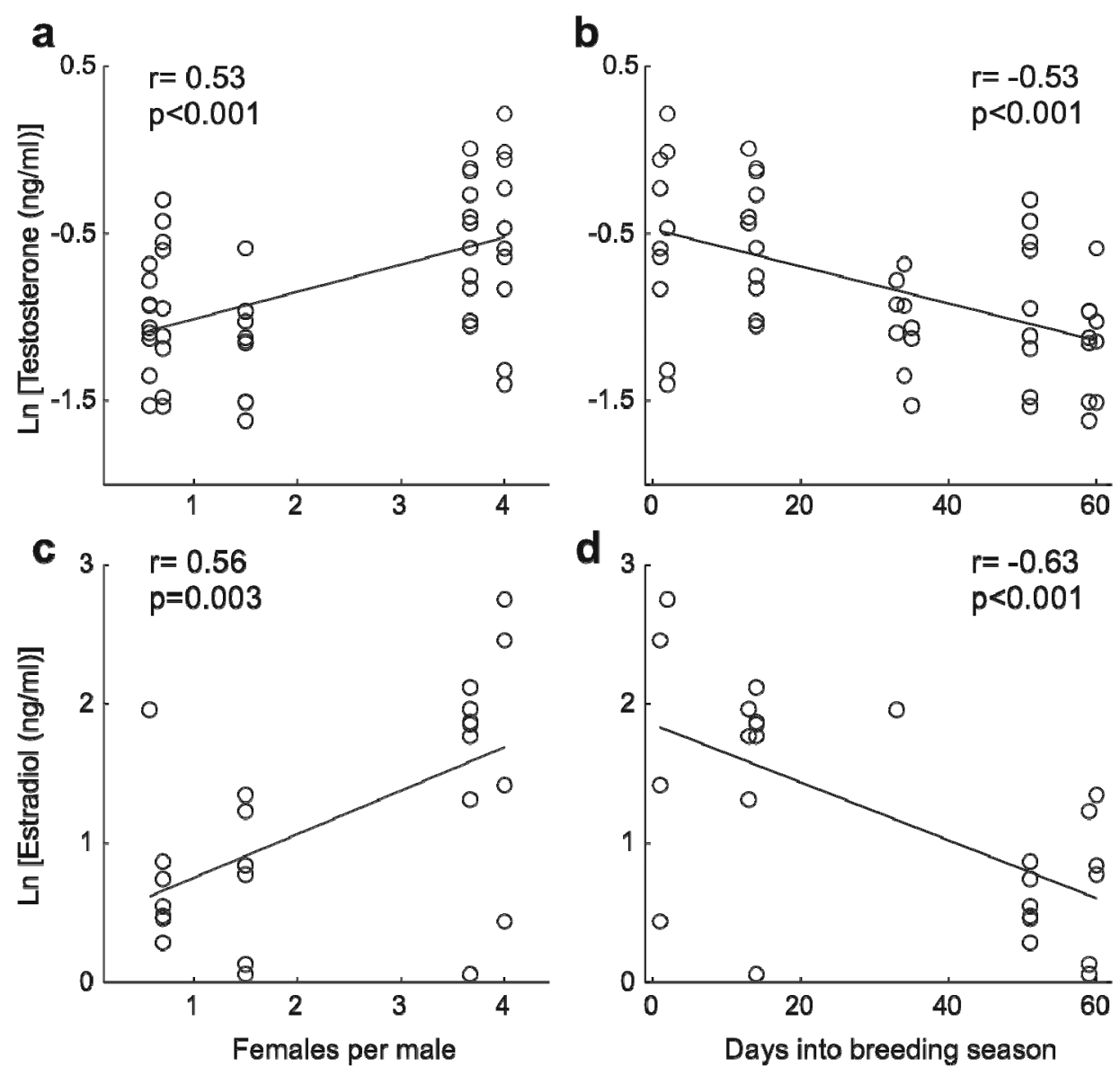

Figure 4.3. Steroid levels in relation to adult sex-ratio (ASR) and time into the breeding season. Circles indicate individual levels while lines indicate the estimated linear relationship. Both testosterone and estradiol levels in the field increased as the number of females per males increased $(\mathbf{a} \& \mathbf{c})$, but decreased with time into the breeding season (b \& d) 

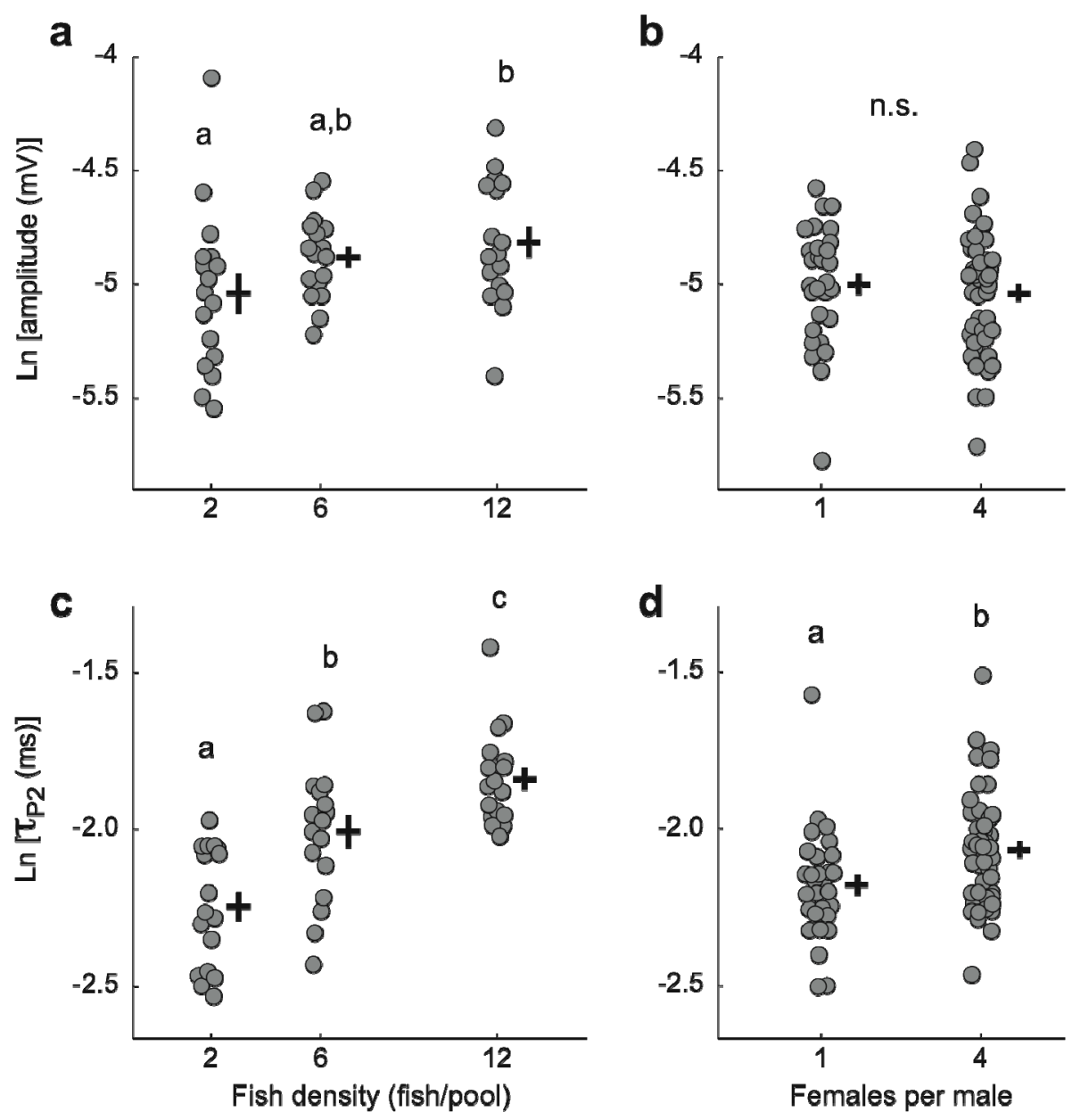

Figure 4.4. Changes in electric organ discharge (EOD) amplitude and EOD duration when fish density and adult sex-ratio (ASR) were independently manipulated in the lab. a, EOD amplitude increased as population density increased. $\mathbf{b}$, EOD amplitude in the lab did not respond to changes in ASR, as oppose to field observations (Fig. 2b). c, EOD second phase duration ( $\left.\tau_{\mathrm{P} 2}\right)$ steadily increased with population density in the lab. $\mathbf{d}$, Females increased EOD $\tau_{\mathrm{P} 2}$ as the ASR became female-biased. Circles depict raw data while crosses represent mean \pm SEM. Pairwise comparisons among groups significant $(P$ $<0.05)$ are shown by lower case letters 


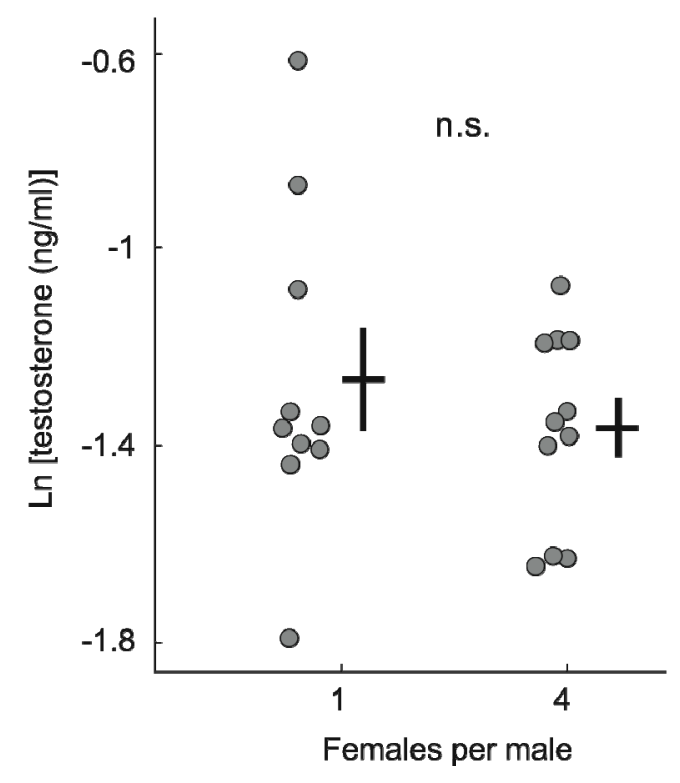

Figure 4.5. Testosterone levels from females at different adult sex-ratio (ASR) in the lab. There were no significant differences in testosterone levels between females at a unity versus a female-biased ASR 


\title{
CHAPTER 5
}

FOOD RESTRICTION PROMOTES SIGNALING EFFORT IN RESPONSE TO SOCIAL CHALLENGE IN A SHORT-LIVED ELECTRIC FISH

\author{
As accepted for publication by Hormones and Behavior \\ July 2012 \\ Copyright $($ E Elsevier
}

DOI: 10.1016/j.yhbeh.2012.07.003 


\begin{abstract}
Vertebrates exposed to stressful conditions release glucocorticoids to sustain energy expenditure. In most species elevated glucocorticoids inhibit reproduction. However individuals with limited remaining reproductive opportunities cannot afford to forgo reproduction and should resist glucocorticoid-mediated inhibition of reproductive behavior. The electric fish Brachyhypopomus gauderio has a single breeding season in its lifetime, thus we expect males to resist glucocorticoid-mediated inhibition of their sexual advertisement signals. We studied stress resistance in male $B$. gauderio (i) by examining the effect of exogenous cortisol administration on the signal waveform and (ii) by investigating the effect of food limitation on androgen and cortisol levels, the amplitude of the electric signal waveform, the responsiveness of the electric signal waveform to social challenge, and the amount of foraging activity. Exogenous cortisol administration did reduce signal amplitude and pulse duration, but endogenous cortisol levels did not rise with food limitation or social challenge. Despite food limitation, males responded to social challenges by further increasing androgen levels and enhancing the amplitude and duration of their electric signal waveforms. Food-restricted males increased androgen levels and signal pulse duration more than males fed ad libitum. Socially challenged fish increased food consumption, probably to compensate for their elevated energy expenditure. Previous studies showed that socially challenged males of this species simultaneously elevate testosterone and cortisol in proportion to signal amplitude. Thus, B. gauderio appears to protect its cortisol-sensitive electric advertisement signal by increasing food intake, limiting cortisol release, and offsetting signal reduction from cortisol with signal-enhancing androgens.
\end{abstract}




\section{Introduction}

The energetic expense of reproduction typically entails reconfiguration of the individual's energy budget. Energetic reallocation is particularly extreme in semelparous species, which breed only once in their lifetimes. Semelparous species favor investment in reproduction over investment in survival during their single breeding season (Williams, 1966). Semelparous vertebrates are typified by a seasonal rise in glucocorticoid (GC) levels, as they divert energy from somatic maintenance into reproduction, (McEwen and Wingfield, 2003; Wingfield and Sapolsky, 2003). Semelparous species with short breeding seasons of few days or weeks show a particularly sharp rise in GC levels concurrent with the onset of reproduction (McDonald et al., 1981; Boonstra and McColl, 2000; Carruth et al., 2000; Barry et al., 2001). However, other semelparous species with longer breeding seasons (a few months) do not increase GC levels during the breeding season (Oakwood et al., 2001). We hypothesize that whether GCs rise precipitously in semelparous species depends on the length of the breeding season itself. Semelparous species with a short breeding season are typically capital breeders, investing all their resources into a single bout of reproduction. On the other hand, semelparous species with a longer breeding season should be more conservative with their energetic allocation using current income to help fuel reproduction while preventing a rise in GCs levels in order to endure throughout the breeding season. We asked whether semelparous species with longer breeding seasons have mechanisms to prevent the rise in GCs and therefore reduce the detrimental effect of GCs on survival, somewhat resembling what occurs in iteroparous species, which breed multiple times throughout their lifetimes. 
Sterling and Eyer (1988) coined the term allostasis to describe the physiological adaptations an organism undertakes to maintain internal stability when external or internal conditions change. Allostatic load is the effort of maintaining homeostasis, the difference between the energy available and the energy needed for physiology and behavior while engaged in the demands of life history: finding mates, breeding, defending territories, surviving, etc. Allostatic load increases when energy available diminishes or when the energetic demands of the organisms increase. Allostatic overload occurs when energetic demands surpass the amount of available energy (rev. McEwen and Wingfield, 2003). To reduce allostatic load, an overloaded individual could increase energy consumption or decrease energy expenditure by reducing the amount of energy allocated to functions not essential for immediate survival, such as reproduction (McEwen and Wingfield, 2003). Since glucocorticoids regulate behavioral and physiological mechanisms related to energy expenditure and intake, their circulating levels are commonly taken as indicators of allostatic load. In most species elevated glucocorticoids result in reproductive inhibition. However, those species for which forgoing reproduction to benefit survival would not increase fitness have evolved mechanisms to resist stress while continuing to reproduce. Following the logic of G.C. Williams (1966), we expect semelparous species to go into allostatic overload during reproduction (Wingfield and Sapolsky, 2003).

We investigated the strategy by which a semelparous vertebrate with a relatively long breeding season fuels and sustains reproductive behavior. Specifically, we studied the effect of increased allostatic load on foraging behavior and reproductive signal production in the electric fish Brachyhypopomus gauderio, and whether the 
advertisement signal of B. gauderio resists the inhibitory effects of cortisol. We consider three possible responses of B. gauderio to allostatic load: (i) Although semelparous species are not expected to forgo reproduction when resources are limited, semelparous species with long breeding seasons could postpone reproduction or reduce reproductive investment for the short term until environmental conditions improve. In this case, a rise in GCs may beneficially inhibit reproductive behaviors and physiology. (ii) Short-lived species with a long breeding season will have mechanisms to reduce allostatic load, such as increasing food supply to sustain reproduction and prevent the rise of GCs. (iii) Alternatively, when allostatic load increases semelparous species with long breeding season might respond like those species with short breeding seasons, through a sharp increase in circulating GCs and direction of all remaining energy capital towards reproduction. In the latter case, a rise in GCs should not inhibit reproductive behaviors or physiology.

The gymnotiform electric fish B. gauderio (southern sister species of $B$. pinnicaudatus) is an excellent model to study the behavioral and physiological effects of allostatic load. B. gauderio reproduces throughout the austral summer in subtropical regions of the southern hemisphere (Silva et al., 2003). Under field conditions, $B$. gauderio is an annual - only immature fish can be found during the austral winter, indicating that adults from the previous year have not survived the breeding season (Silva et al., 2003). Thus, a single reproductive season lasting a few months (Silva et al., 2003), combined with energetically expensive signals (Salazar and Stoddard, 2008; Stoddard and Salazar, 2011) marks males B. gauderio as likely candidates for routine allostatic overload. 
B. gauderio generate electric organ discharges (EODs) 24 hours a day that reflect their hormonal profiles (Stoddard et al., 2006; Gavassa et al., 2011). Male B. gauderio produce EODs of higher magnitude, longer duration and greater energetic cost than females or subadults (Hopkins et al., 1990; Franchina and Stoddard, 1998; Silva et al., 2002; Salazar and Stoddard, 2008). At night and especially when challenged by a conspecific male, males increase EOD amplitude and duration of the second phase (Franchina et al., 2001; Silva et al., 2002; Silva et al., 2007) resulting in a further increase on the energetic expense of signaling (Salazar and Stoddard, 2008; Stoddard and Salazar, 2011). Social competition increases androgen and cortisol levels along with signal enhancement (Salazar and Stoddard, 2009). The concurrent increase in cortisol levels with androgen levels and signal enhancement suggests that the electric signal of $B$. gauderio resists suppression by cortisol.

We tested response of the electric signal of B. gauderio to experimentally elevated cortisol levels. We also manipulated the allostatic load by manipulating energy availability and demand. We reduced the availability of energy by manipulating food availability, and we increased energetic demand by staging social challenges from conspecific males. If B. gauderio is typical of semelparous vertebrates, we might expect to see cortisol elevate at the onset of reproduction while reproductive physiology and signaling behavior continue or increase. Alternatively, since this species has a relatively long breeding season for a semelparous species, food limitation might reveal a capacity for cortisol-mediated suppression of reproduction, such as reduced signal output, or individuals might seek to compensate through increased foraging effort. 


\section{Materials and methods}

\section{$\underline{\text { Animals }}$}

In the experiment manipulating allostatic load we used 48 sexually mature male Brachyhyopomus gauderio (mean \pm s.d.: total length $=19.3 \pm 1.9 \mathrm{~cm}$, weight $=9.97 \pm$ $2.64 \mathrm{~g}$ ) from the captive-bred $18^{\text {th }}$ generation colony at Florida International University. In the cortisol administration experiment we used 36 sexually matured males from the $17^{\text {th }}$ generation of the same colony (total length $=18.0 \pm 1.8 \mathrm{~cm}$, weight $=7.81 \pm 1.88 \mathrm{~g}$ ). We kept fish in 450-liter outdoors pools in mixed-sex groups containing from 6 to 14 fish with water hyacinths, Eichhornia crassipes as natural hiding and spawning sites. We maintained water conductivity in the pools between $70-100 \mu \mathrm{S} \mathrm{cm}^{-1}$. Seasonal rain and temperature fluctuations of South Florida stimulated breeding. We fed the fish live oligochaete blackworms three times a week. Experiments were conducted during the breeding season, May-Sept 2009 and 2010. Experimental procedures were performed under the guidelines and approval of the Institutional Animal Care and Use Committee at Florida International University (protocol 08-027).

Experimental design

\section{Experiment 1: cortisol administration}

Males were isolated in 40-liter tanks for one week, a treatment that standardizes circulating cortisol levels among males (Salazar and Stoddard, 2009). After isolation, males were assigned to receive either cortisol or a vehicle (control). We manipulated cortisol levels by adding cortisol to the water of the fish's tank. Hydrocortisone (98\% HPLC grade, Sigma Aldrich, St Louis, MO) was initially dissolved in 90\% ethanol at a concentration of $15 \mathrm{mg} \mathrm{ml}^{-1}$ and then dissolved in aquarium water to a concentration of 
$150 \mathrm{ng} \mathrm{ml}^{-1}$ for cortisol, which is the upper limit of endogenous cortisol concentration in socially challenged fish (Salazar and Stoddard, 2009), and a final concentration of $10 \mu 11^{-}$ ${ }^{1}$ of ethanol in aquarium water. Fish in the control groups had the same volume of $90 \%$ ethanol dissolved in aquarium water $\left(10 \mu \mathrm{l}^{-1}\right)$. We recorded electric signals for three days after cortisol treatment, while fish remained in the treated water. We took a blood sample to verify the rise in circulating cortisol levels among the treated males. We drew sufficient blood samples from eight cortisol-treated fish and six controls.

\section{Experiment 2: allostatic load manipulation}

We randomly assigned males to one of four treatments: 1) ad libitum (AL) food and social challenge, 2) food restriction (FR) and social challenge, 3) ad libitum food and isolation, and 4) food restriction and isolation. Each male went through one single treatment. The food restriction diet consisted of two blackworms per day. A pilot study showed that males fed ad libitum eat approximately 20 blackworms per day, thus two worms supplied $10 \%$ of the average daily food intake. Before the experiment, we isolated all males for one week. On experiment day 1, we transferred each male to an EOD machine tank, initiated continual EOD recording, and began the $10 \%$ diet for the males in the food restriction treatments. After 10 days of food treatment, a conspecific male of similar size was added to the recording tank of each male in the social challenge treatments for another five days (Fig. 2).

\section{$\underline{\text { EOD recordings }}$}

We recorded EODs in the automated system for calibrated EOD recordings called the EOD machine (Franchina and Stoddard, 1998; Stoddard et al., 2003). The EOD machine automatically records EODs from free-swimming fish and analyzes the EODs 
simultaneously. The EOD machine consists of 12 tanks, each one divided in three compartments by two screen-mesh partitions. An unglazed ceramic tube connects the two mesh partitions. The focal fish resides in the two outer compartments. When the fish rests in or swims through the ceramic tube connecting those compartments, a set of electrodes placed on top of the tube detects the fish's position and triggers a recording of the EOD from a pair of nichrome electrodes located at both ends of the tank. EODs are amplified 500X and low-pass filtered. The EOD parameters analyzed are (1) EOD amplitude, calculated as the sum of the peak amplitude of each phase, and (2) the duration of the EOD's $2^{\text {nd }}$ phase estimated by the variable $t_{\mathrm{P} 2}$, the time constant of the repolarization of the second phase (Fig. 1). When used, a stimulus fish was placed in the inner compartment, where it could interact electrically with the focal male through the electrically transparent mesh, but with no direct mechanical contact.

\section{Blood collection and analysis}

For males in the allostatic manipulation experiment, we sampled blood on the last day of recordings (day 15), between 10:00 and 11:00. For fish in the cortisol administration experiment, we sampled blood a week after the start of cortisol treatment, between 14:00 and 15:00. Prior to drawing blood, fish were anesthetized in a fast acting eugenol solution $\left(0.8 \mathrm{mg}^{-1}\right)$. We drew blood from the subveretebral sinus into a syringe with $5 \mu 1$ of $10 \%$ EDTA. The sample was then centrifuged at $7000 \mathrm{rpm}$ for 15 minutes. Plasma was stored at $-80^{\circ} \mathrm{C}$ until analysis. Plasma samples were collected, extracted and analyzed following a validated protocol for this species (Salazar and Stoddard, 2009). Plasma was diluted 1:100 using the buffer provided by the immunoassay manufacturer (Cayman Chemical Inc. Ann Arbor, MI). We extracted $250 \mu \mathrm{l}$ of diluted plasma four times in a mixture of 
7:3 hexane:ethyl-acetate for cortisol (F) and testosterone (T), and 9:1 hexane:ethylacetate for 11-ketotestosterone (11-KT). All samples were plated in duplicate as specified by the kit manufacturer. To verify extraction recovery, we extracted EIA kit standards alongside the plasma samples. For the allostatic load manipulation experiment we used one plate for each hormone assay. Unfortunately, isolated fish had very low testosterone concentration that fell bellow the detection limit of the assay, and we did not have another plate to re-run the samples at a higher concentration. The detection limits of the EIA kits reported by the manufacturer were $35 \mathrm{pg} \mathrm{ml}^{-1}$ for $\mathrm{F}, 6 \mathrm{pg} \mathrm{ml}^{-1}$ for $\mathrm{T}$, and $1.3 \mathrm{pg}$ $\mathrm{ml}^{-1}$ for $11-\mathrm{KT}$. The intra-assay coefficients of variation were: $5.54 \%$ for $\mathrm{F}, 5.29 \%$ for $\mathrm{T}$, and $6.88 \%$ for $11-\mathrm{KT}$. For the cortisol administration experiment, the intra-assay coefficient of variation for the cortisol plate was $3.65 \%$.

Data analysis

We log transformed all variables to fulfill the normality assumption and verified normality using Shapiro-Wilk's test. For experiment 1 we used a t-test to analyze the differences in EOD parameters between cortisol-treated and control males. For experiment 2 we divided the analysis in two parts: first we tested the effect of diet on the EOD waveform and body weight before social treatment, comparing the changes that occurred between days 1 and 10 of the experiment. Then we tested the effect of social treatment combined with diet by comparing the percentage change in EOD waveform and body weight between days 10 and 15 of the experiment. We analyzed the effect of social treatment and diet using a two-way ANOVA, with two factors and two levels each: 1) diet treatment (AL vs. FR), and 2) social treatment (social vs. isolated). We used Tukey post-hoc test to determine significant pairwise differences. We used nonparametric 
analysis to study changes in worm consumption since the average number of worms eaten per day could not be normally transformed. We used Wilcoxon sign test to test for differences in worm consumption within each social treatment. We used a Wilcoxon rank sum test to test for differences in average worms eaten between social and isolated treatments, both before and after social treatments. Since the treatments were compared twice (days 1-10 and days 10-15) we used a Bonferroni-corrected $\alpha=0.025$. All analyses were performed using MATLAB Statistical Toolbox and SPSS v.18.

\section{Results}

Effect of cortisol in the amplitude of the EOD

Cortisol administration in the water elevated the levels of circulating cortisol in treated fish over untreated controls ( $t$-test: $\mathrm{t}=-2.83, \mathrm{p}=0.015$ d.f. $=12$; Fig. $3 \mathrm{~A}$ ) within the physiological range. Cortisol administration decreased both amplitude and the duration of the EOD in treated fish relative to untreated controls (amplitude: $t$-test $t=2.90, p=0.006$, d.f.=33; duration: t-test $\mathrm{t}=2.34, \mathrm{p}=0.025$, d.f. $=33$; Fig. 3B,C). Thus, cortisol on its own has a suppressive effect on the EOD waveform. However, we found no significant correlation between circulating levels of cortisol and change in signal parameters (cortisol vs. amplitude change in untreated fish: $r=0.23, p=0.65$; cortisol vs. amplitude change in cortisol-treated fish: $\mathrm{r}=0.28, \mathrm{p}=0.51$; cortisol vs. duration change in untreated fish: $r=0.59, p=0.21$; cortisol vs. duration change in cortisol-treated fish: $r=-0.33, p=0.42$, Pearson correlations).

\section{Effect of food restriction on the electric signal}

We tested the effect of 10 days of food restriction on EOD parameters before adding the social stimulus. We found no significant difference in the percentage change 
in EOD amplitude between fish in ad libitum diet and food restricted diets (day EOD amplitude $\mathrm{t}$-test: $\mathrm{t}=0.58, \mathrm{p}=0.57$ d.f. $=44$; night $\mathrm{EOD}$ amplitude $\mathrm{t}$-test: $\mathrm{t}=0.42, \mathrm{p}=0.67$ d.f.=44; Fig. 4A). Neither did we find a significant difference in the percentage change in EOD duration between the two diet treatments (day EOD duration $t$-test: $t=-0.25, p=0.80$ d.f. $=44$; night EOD duration t-test: $\mathrm{t}=-1.11$, $\mathrm{p}=0.27$ d.f.=44; Fig. 4B).

\section{Effect of food restriction and social challenge on the electric signal}

Fish in both food treatments increased the amplitude and duration of their electric signals when exposed to a conspecific male. However, in response to social stimulus, fish in the food restricted treatment increased the amplitude of their EOD more than fish fed ad libitum (day amplitude change: social effect: $\mathrm{F}_{1,42}=51.51, \mathrm{p}<0.001$, diet effect: $F_{1,42}=3.24 p=0.079$, interaction: $F_{1,42}=1.48 p=0.23$; night amplitude change: social effect: $F_{1,42}=68.42, p<0.001$, diet effect: $F_{1,42}=5.87 p=0.019$, interaction: $F_{1,42}=3.71 p=0.061$; Fig. 5A). Likewise, fish in the FR treatment increased EOD duration more in response to social stimulation than AL fish. However, FR males that remained in isolation show changes in EOD duration comparable to AL males with social treatment (day EOD duration change: social effect: $F_{1,42}=20.19, p<0.001$, diet effect: $F_{1,42}=4.64 p=0.037$, interaction: $F_{1,42}<0.01 p=0.95$; night EOD duration change: social effect: $F_{1,42}=20.75$, $p<0.001$, diet effect: $F_{1,42}=7.74 p=0.008$, interaction: $F_{1,42}=0.97 p=0.33$; Fig. 5B). Effect of food restriction and social challenge on the hormonal profile

The differences in EOD duration reflect differences in 11-ketotestosterone levels. Males in the social treatments had higher 11-KT levels, despite food treatment, while males that remained in isolation and food restriction had 11-KT levels in between those 
of social challenged fish and isolated fish with ad libitum food (social effect: $\mathrm{F}_{1,31}=8.96$, $p=0.005$, diet effect: $F_{1,31}=0.68 p=0.41$, interaction $F_{1,31}=0.46 p=0.51$; Fig. $6 B$ ). Neither food restriction nor social challenge had a detectable effect on circulating levels of testosterone or cortisol (testosterone social effect: $\mathrm{F}_{1,25}=1.88, \mathrm{p}=0.18$, diet effect: $F_{1,25}=0.02 p=0.88$, interaction: $F_{1,25}=2.74 p=0.11$; cortisol social effect: $F_{1,31}=0.08$, $p=0.78$, diet effect: $F_{1,31}=1.36, p=0.25$, interaction: $F_{1,31}=0.46, p=0.50$; Fig. 6 A,C). Effect of food restriction and social challenge on body condition and feeding behavior

Although all male groups lost weight before social stimulus was added, some males in AL diet maintained their weight and some even gained weight, while no male in FR diet gained nor maintained his weight (2-tailed t-test: $\mathrm{t}=2.67, \mathrm{df}=45, \mathrm{p}=0.01$; Fig. $7 \mathrm{~A}$ ). Following the social challenge, males receiving the social treatment with ad libitum diet held their weight better than all the other treatments (2-way ANOVA, Social effect: $F_{1,41}=9.96, p<0.01$, diet effect: $F_{1,41}=5.13, p=0.03$, interaction: $F_{1,41}=9.08, p=0.004 ;$ Fig. 7B). Social challenge seems to increase feeding behavior, at least in the FR groups, where we counted the number of worms consumed, socially challenged males ate more worms after the social challenge while the males that remained in isolation for the same period of time ate fewer worms (within group comparison worms eaten in days 1-10 to worms eaten days $10-15$, Wilcoxon sign test, isolated $\mathrm{p}=0.31$, social $\mathrm{p}=0.039$; between group comparison worms eaten in social vs. isolated, Wilcoxon rank test, days 1-10: $\mathrm{p}=0.732 *$, days $10-15 \mathrm{p}=0.004 *$; *Bonferroni corrected $\alpha=0.025$ Fig. 8 ). 


\section{Discussion}

$\underline{\text { Response to allostatic load in the context of life history theory }}$

Semelparous species should resist stress and continue to reproduce during stressful periods while iteroparous species should postpone reproduction in response to stress and focus on survival (Wingfield and Sapolsky, 2003). However, semelparous species vary in duration of their breeding season from just a few days to a few months. We studied how food restriction affects the energetically demanding signal of a semelparous species with a relatively long breeding season. Male $B$. gauderio in our study could have responded to the increased allostatic load in one of three ways: (i) reducing the load by lowering energetic expense by decreasing signaling effort, thereby compromising reproduction, (ii) "pay as you go", reducing the load by foraging more to increase the energy supply, preventing a rise in GCs and sustaining the signal, or (iii) "go for broke", burning energy stores to fuel reproductive signaling resulting in a negative energy balance. In response to increasing allostatic load B. gauderio males did not reduce energy output by diminishing their signals; in fact the biggest signals came from food-restricted males exposed to social challenge. Males signaling at high intensity foraged more when that was possible (higher food availability), or in the absence of food, simply burned faster through their remaining energy stores. Males sustained reproductive signaling, varying their fueling strategy depending on food availability. When food was plentiful, males opted for a "pay as you go" strategy to fuel signaling; when food was scarce, males switched to a "go for broke" strategy. Social challenge induced male $B$. gauderio to increase their energetically expensive electric signal parameters, especially when under energetic stress from dietary restriction. This general pattern of investment in signals is consistent with the "go for 
broke" energetic strategy expected of animals facing time-limited reproductive opportunity, and contrasts with the "postpone" strategy of animals that can forestall reproduction until more favorable conditions reappear. However, cortisol levels did not rise even in animals that had to burn energy stores to fuel reproduction.

\section{Alternative 1: Postpone reproduction}

According to the Energetics-Hormone Vocalization (EHV) model (Emerson, 2001), signaling output should decrease as energy reserves decline. The EHV model proposes that energetically expensive signaling would elevate circulating androgens, as predicted by the Challenge Hypothesis (Wingfield et al., 1990), but it would also elevate GCs to fuel signaling. Prolonged signaling will decrease energetic stores until GCs rise to a level at which they inhibit androgen release. The resultant drop in circulating androgen then causes signaling output to decline (Emerson, 2001; Moore and Jessop, 2003). For instance, male barking tree frogs (Hyla gratiosa) increase the number of nights they attend a chorus when supplemented with extra food (Murphy, 1994), although food supplementation has no effect on the amount of time spent at the chorus on a given night (Murphy, 1999). Likewise, male fiddler crabs (Uca lactea), though not regulated by androgens and GCs, nevertheless increased courting effort on the day after food supplementation (Kim et al., 2008). Food limitation prevents signal exaggeration of low quality male sticklebacks (Gasterosteus aculeatus) (Candolin, 2000). Conversely, in male green anole lizards (Anolis carolinensis), food limitation does not compromise signal expression (dewlap size) but it does compromise the advertised trait expression (bite force) (Lailvaux et al., 2012). Iteroparous species seem to compromise traits important for reproduction when under food limitation (Moore and Jessop, 2003). Barking tree 
frogs, sticklebacks, and green anoles are iteroparous species that can live up to several years. Conversely, as expected from their semelparous life-history, B. gauderio males in the food restriction treatments did not compromise their signaling output compared to males with unrestricted food (Fig. 4).

Alternative 2A: Prevent GC rise by compensatory feeding

To protect their reproductive signaling output, males can reduce allostatic load and prevent GC rise by increasing energy intake. In our experiment, B. gauderio in the FR diet increased foraging behavior to compensate for additional energetic expenditure of EOD enhancement during social challenge. Within the food-restricted treatments, social interactions stimulated worm consumption (Fig. 7). Although we did not count the number of worms consumed by males in the ad libitum treatments, we did find that males in the ad libitum treatment with social challenge lost less weight than males in other treatments (some even gained weight), possibly a result of differential foraging activity (Fig. 6b). This finding was unexpected, since in most species males trade off between time spent signaling and time spent foraging (Fernald and Hirata, 1977; Nolan Jr, 1978; Abrahams, 1993; Griffiths, 1996; Lindstrom et al., 2009). For many taxa, male sexual displays are incompatible with feeding behavior and in others, spatial separation between preferred feeding and signaling grounds prevents simultaneous foraging and reproductive signaling. However, B. gauderio can display their enhanced electric signal and feed simultaneously.

Unexpectedly, fish with AL diet lost weight during isolation. It appears that social interaction is necessary to stimulate feeding and prevent weight loss in B. gauderio. This may explain why fish in FR diet under isolation and social conditions lost similar weight, 
the former probably due to lack of appetite while the latter lost weight due to lack of food availability. The increase in worm consumption even with a restricted diet, might have prevented a further weight loss in FR social fish. Alternatively, isolation may have additional effects on weight loss besides lack of appetite. For instance, the social environment can cause fish to either grow or shrink even when food supply is equal (Hofmann et al., 1999). We have noticed that our isolated fish tend to reduce the size of their caudal filaments.

\section{Alternative 2B: Prevent GC rise by physiological adaptation}

EOD regulation in B. gauderio involves the interplay between hormones of the hypothalamus-pituitary-interrenal (HPI) and the hypothalamus-pituitary-gonadal (HPG) axes. Social stimulation increases both EOD parameters over two time scales: 1) Rapid increases in EOD parameters are induced in minutes by direct action of HPI melanocortins (adrenocorticotropic hormone, ACTH; and alpha-melanocyte stimulating hormone, $\alpha-\mathrm{MSH}$ ). Melanocortins act directly on electrocytes (electrogenic cells) through the intracellular cAMP-PKA pathway (Markham and Stoddard, 2005; Stoddard, 2007; Markham et al., 2009). 2) Slower increases in EOD parameters are mediated by androgens. Androgen implants greatly increase the amplitude and the duration of the EOD's second phase after three days (Stoddard et al., 2006; Allee et al., 2009; Goldina et al., 2011), and endogenous levels of circulating testosterone (T) and 11-ketotestoterone (11-KT) are tightly related to the EOD's second phase duration (Gavassa et al., 2011). Androgens are also known to augment the waveforms of other electric fish taxa (Hagedorn and Carr, 1985; Bass and Volman, 1987; Landsman et al., 1990; Dunlap et al., 1998; Herfeld and Moller, 1998; Zakon et al., 1999; Silva et al., 2002; McAnelly et al., 
2003), possibly by altering the density and kinetics of ion channels (Bass and Hopkins, 1983; Carlson et al., 2000; Bass and Zakon, 2005). Furthermore in B. gauderio, rapid and slow mechanisms have synergistic effects on the EOD: androgens potentiate the effect of melanocortins on both EOD amplitude and the duration of the second phase (Allee et al., 2009; Goldina et al., 2011). Action of androgens and melanocortins fully account for the enhancing effects of a social challenge on duration of the EOD's second phase, though their experimental administration has smaller effects on EOD amplitude (Goldina et al., 2011) leaving open the possibility that another hormone also regulates EOD amplitude.

Paradoxically, male B. gauderio produce larger, and thus more expensive signals under periods of energy imbalance typically associated with strong release of glucocorticoids. These results allow us to consider Wingfield and Sapolsky's (2003) five possible mechanisms for resisting cortisol-mediated deactivation of reproductive behavior. The first two deal with preventing a rise in GCs: (i) HPA/HPI activation is prevented at the level of the central nervous system precluding the stressor to be perceived as stressful. As mentioned above, the increase in EOD parameters requires release of HPI melanocortin hormones. Therefore, the enhancement of the EOD reveals HPI activation. However, both ACTH and a-MSH stimulated EOD enhancement (Markham and Stoddard, 2005; Markham et al., 2009; Goldina et al., 2011), but commonly only ACTH stimulates cortisol release (Wendelaar Bonga and Balm, 1995). Whether B. gauderio can limit ACTH release while permitting a-MSH release is unknown. (ii) blockage of the HPA/HPI axis glucocorticoid release pathway. The lack of a significant cortisol increase in food-restricted and socially challenged males is consistent with such a blockage. However in prior studies that simulated higher social 
competition than the experiment presented here (five challenging males vs. one challenging male), cortisol increased simultaneously with increased EOD parameters (Salazar and Stoddard, 2009) despite the finding presented here that cortisol by itself reduces EOD parameters. Thus, $B$. gauderio may only block cortisol release during intermediate levels of competition. B. gauderio appears to use different stress-resistance mechanisms depending on the intensity of the stressor. Alternatively, our food restriction and social treatments were not strong enough to evoke a rise in cortisol levels.

\section{Alternative 3: simultaneous increase of GC and reproductive signaling}

Although we did not find a rise in cortisol in this study, males exposed to more intense social competition than what we presented here, show elevated levels of cortisol (Salazar and Stoddard, 2009). If male B. gauderio routinely experience sustained elevations of cortisol during periods of intense social competition (e.g., early in the breeding season), they should have a mechanism in place to prevent cortisol-mediated inhibition of reproductive signaling. In the previous section we explored mechanisms that prevented cortisol rise. The last three mechanisms for resisting cortisol-mediated deactivation of reproductive behavior proposed by Wingfield and Sapolsky's (2003) deal with blocking GC-mediated inhibition of reproductive physiology:(iii) resistance of the hypothalamic-gonadal axis (HPG) to the suppressive effects of glucocorticoids. We found that direct cortisol administration decreased EOD amplitude (Fig. 3). Thus, our experimental reduction of EOD parameters with physiological levels of cortisol rules out this mechanism. However, we found no correlation between cortisol levels and change in signal parameters. The time elapsed between signal recordings and blood collection might have allowed signals to dissociate from cortisol levels. (iv) compensatory 
activation of the gonadal axis to offset glucocorticoid suppression. Consistent with this mechanism, circulating cortisol correlates with EOD amplitude and circulating testosterone (Salazar and Stoddard, 2009), while both T and 11-KT increased in males on the food-restricted treatments. Interestingly, hormones from the HPA/HPI can be responsible for compensatory HPG activation. For example in the Arctic ground squirrel, ACTH injection promotes testosterone release in males (Boonstra and McColl, 2000). (v) protection from corticosteroid-binding proteins by sequestering free glucocorticoids.

Since we measured only total steroids, we cannot address this mechanism. Our results indicate that B. gauderio resists stress by preventing cortisol accumulation, compensatory activation of the HPG axis (increase in androgen production), and direct action of HPI hormones promoting reproductive behaviors.

\section{Conclusion}

Resistance to glucocorticoid inhibition on reproduction has been observed in semelparous species in which glucocorticoid levels soar during the breeding season, for example sockeye salmon (Oncorhynchus nerka) (Carruth et al., 2000; Barry et al., 2001), arctic ground squirrels (Spermophilus parryii plesius) (Boonstra and McColl, 2000; Boonstra et al., 2001), and marsupial species from the genera Antechinus and Phascogale (Bradley et al., 1980; McDonald et al., 1981; Bradley, 1997). All of these species have a very short breeding season lasting from a few days (sockeye salmon) to a couple weeks (arctic squirrels and marsupials). However, the marsupial Dasyurus hallucatus shows no rise in cortisol levels during the breeding season (Oakwood et al., 2001), even though its lifehistory is very similar to Antechinus and Phascogale species, where all males die after a single breeding season. However, the breeding season of Dasyurus hallucatus lasts 3-4 
months, similar to the length of the breeding season in B. gauderio, and much longer than the breeding season of Antechinus and Phascogale species (2-3 weeks) (Bradley et al., 1980; McDonald et al., 1981; Bradley, 1997; Oakwood et al., 2001).

We do not believe that B. gauderio is glucocorticoid resistant since we did not see a significant increase in cortisol levels with allostatic load, and because exogenous cortisol administration diminishes EOD parameters. Instead B. gauderio appears to prevent the rise in glucocorticoids when possible, or compensates with enhanced androgen release (Salazar and Stoddard, 2009). A more severe diet treatment and social challenge than what we performed here is necessary to test whether B. gauderio prevents cortisol increase or compensates cortisol effects by other mechanisms.

The relatively long breeding season of B. gauderio may favor a stress resistance mechanism that avoids the deleterious effects of elevated GC, extending survival throughout the breeding season. However, when survival conflicts with sustained signaling, B. gauderio would benefit from switching to a 'go for broke' strategy typical of semelparous species with short breeding season. The longer EOD duration and higher androgen levels observed in food restricted fish make us hypothesize that food availability acts as a cue for a decline in future reproductive opportunities, forcing males to increase immediate reproductive investment, as revealed by their high signaling output and androgen levels when food was limited. Life-history is the main determinant of how reproduction is regulated. This study illustrates the great diversity of mechanisms for stress resistance even within species that share a semelparous life-history. 


\section{Acknowledgments}

We thank C. Curtis, A. Goldina, E. Gonzalez, J. Molina, P. Perez and J. Roach for assistance in the lab. We thank two anonymous reviewers for their insightful comments on this manuscript. This work was supported by FIU University Graduate School's Dissertation Evidence Acquisition Fellowship and Dissertation Year Fellowship to S.G., and NSF grant IOS 0956603 to P.K.S.

\section{References}

Abrahams, M. V., 1993. The trade-off between foraging and courting in male guppies. Anim. Behav. 45, 673-681.

Allee, S. J., Markham, M. R., Stoddard, P. K., 2009. Androgens enhance plasticity of an electric communication signal in female knifefish, Brachyhypopomus pinnicaudatus. Horm. Behav. 56, 264-273.

Barry, T. P., Unwin, M. J., Malison, J. A., Quinn, T. P., 2001. Free and total cortisol levels in semelparous and iteroparous chinook salmon. J. Fish Biol. 59, 16731676.

Bass, A. H., Hopkins, C. D., 1983. Hormonal control of sexual differentiation: changes in electric organ discharge waveform. Science. 220, 971-4.

Bass, A. H., Volman, S. F., 1987. From behavior to membranes: testosterone-induced changes in action potential duration in electric organs. Proc. Natl. Acad. Sci. U. S. A. 84, 9295-9298.

Bass, A. H., Zakon, H. H., 2005. Sonic and electric fish: At the crossroads of neuroethology and behavioral neuroendocrinology. Horm. Behav. 48, 360-372.

Boonstra, R., McColl, C. J., 2000. Contrasting stress response of male arctic ground squirrels and red squirrels. J. Exp. Zool. 286, 390-404.

Boonstra, R., McColl, C. J., Karels, T. J., 2001. Reproduction at all costs: The adaptive stress response of male Arctic ground squirrels. Ecology. 82, 1930-1946.

Bradley, A. J., 1997. Reproduction and life history in the red-tailed phascogale, Phascogale calura (Marsupialia: Dasyuridae): the adaptive-stress senescence hypothesis. J. Zool. 241, 739-755. 
Bradley, A. J., McDonald, I. R., Lee, A. K., 1980. Stress and mortality in a small marsupial (Antechinus stuartii, Macleay). Gen. Comp. Endocrinol. 40, 188-200.

Candolin, U., 2000. Changes in expression and honesty of sexual signalling over the reproductive lifetime of sticklebacks. Proc. R. Soc. Lond. B. 267, 2425-2430.

Carlson, B. A., Hopkins, C. D., Thomas, P., 2000. Androgen correlates of socially induced changes in the electric organ discharge waveform of a mormyrid fish. Horm. Behav. 38, 177-186.

Carruth, L. L., Dores, R. M., Maldonado, T. A., Norris, D. O., Ruth, T., Jones, R. E., 2000. Elevation of plasma cortisol during the spawning migration of landlocked kokanee salmon (Oncorhynchus nerka kennerlyi). Comp. Biochem. Phys. C. 127, 123-131.

Dunlap, K. D., Thomas, P., Zakon, H. H., 1998. Diversity of sexual dimorphism in electrocommunication signals and its androgen regulation in a genus of electric fish, Apteronotus. J. Comp. Physiol. A. 183, 77-86.

Emerson, S. B., 2001, Male advertisement calls: behavioral variation and physiological processes. In: M. J. Ryan, (Ed.), Anuran Communication. Smithsonian Institution Press, Washington, DC, pp. 36-44.

Fernald, R. D., Hirata, N. R., 1977. Field study of Haplochromis burtoni: Quantitative behavioural observations. Anim. Behav. 25, 964-975.

Franchina, C. R., Salazar, V. L., Volmar, C. H., Stoddard, P. K., 2001. Plasticity of the electric organ discharge waveform of male Brachyhypopomus pinnicaudatus II. Social effects. J. Comp. Physiol. A. 187, 45-52.

Franchina, C. R., Stoddard, P. K., 1998. Plasticity of the electric organ discharge waveform of the electric fish Brachyhypopomus pinnicaudatus. I. Quantification of day-night changes. J. Comp. Physiol. A. 183, 759 - 768.

Gavassa, S., Silva, A. C., Stoddard, P. K., 2011. Tight hormonal phenotypic integration ensures honesty of the electric signal of male and female Brachyhypopomus gauderio. Horm. Behav. 60, 420-426.

Goldina, A., Gavassa, S., Stoddard, P. K., 2011. Testosterone and 11-ketotestosteone have different regulatory effects on electric communication signals of male Brachyhypopomus gauderio. Horm. Behav. 60, 139-147. 
Griffiths, S. W., 1996. Sex differences in the trade-off between feeding and mating in the guppy. J. Fish Biol. 48, 891-898.

Hagedorn, M., Carr, C., 1985. Single electrocytes produce a sexually dimorphic signal in South American electric fish, Hypopomus occidentalis (Gymnotiformes, Hypopomidae). J. Comp. Physiol. A. 156, 511-523.

Herfeld, S., Moller, P., 1998. Effects of 17 alpha-methyltestosterone on sexually dimorphic characters in the weakly discharging electric fish, Brienomyrus niger (Günther, 1866) (Mormyridae): electric organ discharge, ventral body wall indentation, and anal-fin ray bone expansion. Horm. Behav. 34, 303-319.

Hofmann, H. A., Benson, M. E., Fernald, R. D., 1999. Social status regulates growth rate: Consequences for life-history strategies. Proceedings of the National Academy of Sciences. 96, 14171-14176.

Hopkins, C. D., Comfort, N. C., Bastian, J., Bass, A. H., 1990. Functional analysis of sexual dimorphism in an electric fish, Hypopomus pinnicaudatus, order Gymnotiformes. Brain Behav. Evolut. 35, 350-67.

Kim, T. W., Sakamotom, K., Henmi, Y., Choe, J. C., 2008. To court or not to court: reproductive decisions by male fiddler crabs in response to fluctuating food availability. Behav. Ecol. Sociobiol. 62, 1139-1147.

Lailvaux, S. P., Gilbert, R. L., Edwards, J. R., 2012. A performance-based cost to honest signalling in male green anole lizards (Anolis carolinensis). Proceedings of the Royal Society B: Biological Sciences.

Landsman, R. E., Harding, C. F., Moller, P., Thomas, P., 1990. The effects of androgens and estrogen on the external morphology and electric organ discharge waveform of Gnathonemus petersii (Mormyridae, Teleostei). Horm. Behav. 24, 532-553.

Lindstrom, J., Pike, T. W., Blount, J. D., Metcalfe, N. B., 2009. Optimization of resource allocation can explain the temporal dynamics and honesty of sexual signals. Am. Nat. 174, 515-525.

Markham, M., Allee, S., Goldina, A., Stoddard, P., 2009. Melanocortins regulate the electric waveforms of gymnotiform electric fish. Horm. Behav. 55, 306-313.

Markham, M. R., Stoddard, P. K., 2005. Adrenocorticotropic hormone enhances the masculinity of an electric communication signal by modulating the waveform and timing of action potentials within individual cells. J. Neurosci. 25, 8746-8754. 
McAnelly, L., Silva, A., Zakon, H. H., 2003. Cyclic AMP modulates electrical signaling in a weakly electric fish. Journal of Comparative Physiology a-Neuroethology Sensory Neural and Behavioral Physiology. 189, 273-282.

McDonald, I. R., Lee, A. K., Bradley, A. J., Than, K. A., 1981. Endocrine changes in dasyurid marsupials with differing mortality patterns. Gen. Comp. Endocrinol. 44, 292-301.

McEwen, B. S., Wingfield, J. C., 2003. The concept of allostasis in biology and biomedicine. Horm. Behav. 43, 2-15.

Moore, I. T., Jessop, T. S., 2003. Stress, reproduction, and adrenocortical modulation in amphibians and reptiles. Horm. Behav. 43, 39-47.

Murphy, C. G., 1994. Determinants of chorus tenure in barking treefrogs (Hyla gratiosa). Behav. Ecol. Sociobiol. 34, 285-294.

Murphy, C. G., 1999. Nightly timing of chorusing by male barking tree frogs (Hyla gratiosa): the influence of female arrival and energy. Copeia. 2, 333-347.

Nolan Jr, V., 1978. Ecology and behavior of the prairie warbler Dendroica discolor. American Ornithologists' Union, Washington, DC.

Oakwood, M., Bradley, A. J., Cockburn, A., 2001. Semelparity in a large marsupial. Proc. R. Soc. Lond. B. 268, 407-411.

Salazar, V. L., Stoddard, P. K., 2008. Sex differences in energetic costs explain sexual dimorphism in the circadian rhythm modulation of the electrocommunication signal of the gymnotiform fish Brachyhypopomus pinnicaudatus. J. Exp. Biol. 211, 1012-1020.

Salazar, V. L., Stoddard, P. K., 2009. Social competition affect electric signal plasticity and steroid hormone levels in the gymnotiform fish Brachyhypopomus gauderio. Horm. Behav. 56, 399-409.

Silva, A., Perrone, R., Macadar, O., 2007. Environmental, seasonal, and social modulations of basal activity in a weakly electric fish. Physiol. Behav. 90, 525536.

Silva, A., Quintana, L., Ardanaz, J. L., Macadar, O., 2002. Environmental and hormonal influences upon EOD waveform in gymnotiform pulse fish. J. Physiology-Paris. $96,473-484$. 
Silva, A., Quintana, L., Galeano, M., Errandonea, P., 2003. Biogeography and breeding in gymnotiformes from Uruguay. Environ. Biol. Fish. 66, 329-338.

Sterling, P., Eyer, J., 1988, Allostasis: a new paradigm to explain arousal pathology. In: S. Fisher, J. Reason, (Eds.), Handbook of life stress, cognition and health. John Wiley \& Sons, Chichester, pp. 750.

Stoddard, P. K., 2007, Plasticity of the electric organ discharge waveform: contexts, mechanisms, and implications for electrocommunication. In: F. Ladich, S. P. Collin, P. Moller, B. G. Kapoor, (Eds.), Communication in Fishes. Science Publisher, Inc., Enfield, NH, USA, pp. 623-646.

Stoddard, P. K., Markham, M. R., Salazar, V. L., 2003. Serotonin modulates the electric waveform of the gymnotiform electric fish Brachyhypopomus pinnicaudatus. J. Exp. Biol. 206, 1353-1362.

Stoddard, P. K., Salazar, V. L., 2011. Energetic cost of communication. J. Exp. Biol. 214, 200-205.

Stoddard, P. K., Zakon, H. H., Markham, M. R., McAnelly, L., 2006. Regulation and modulation of electric waveforms in gymnotiform electric fish. J. Comp. Physiol. A. $192,613-624$.

Wendelaar Bonga, S. E., Balm, P. H. M., 1995. The involvement of ACTH and MSH in the stress-response in teleost fish. Netherlands Journal of Zoology. 45, 103-106.

Williams, G. C., 1966. Natural Selection, the costs of reproduction, and a refinement of Lack's Principle. Am. Nat. 100, 687-690.

Wingfield, J., Hegner, R. E., Dufty Jr, A. M., Ball, G., 1990. The "challenge hypothesis": theoretical implications for patterns of testosterone secretion, mating systems, and breeding strategies. Am. Nat. 136, 829-846.

Wingfield, J. C., Sapolsky, R. M., 2003. Reproduction and resistance to stress: when and how. J. Neuroendocrinol. 15, 711-724.

Zakon, H. H., McAnelly, L., Smith, T. G., Dunlap, K., Lopreato, G., Oestreich, J., Few, W. P., 1999. Plasticity of the electric organ discharge: implications for the regulation of ionic currents. J. Exp. Biol. 202, 1409 - 1416. 


\section{Figures and tables}

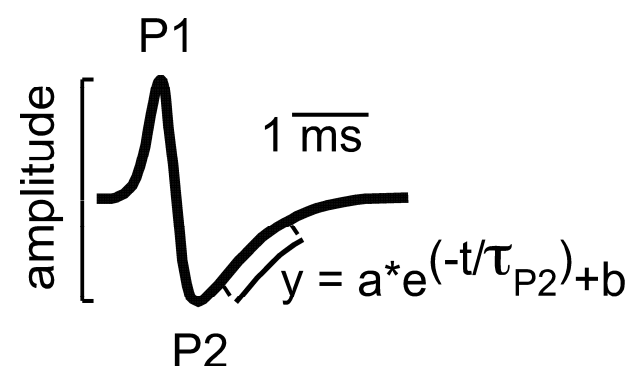

Figure 5.1. The EOD of B. gauderio is a biphasic pulse. We analyzed EOD amplitude by measuring the voltage difference between the positive and negative peaks. We estimated the duration of the signal using the time constant of the repolarization of the EOD's $2^{\text {nd }}$ phase $\left(t_{\mathrm{P} 2}\right)$.

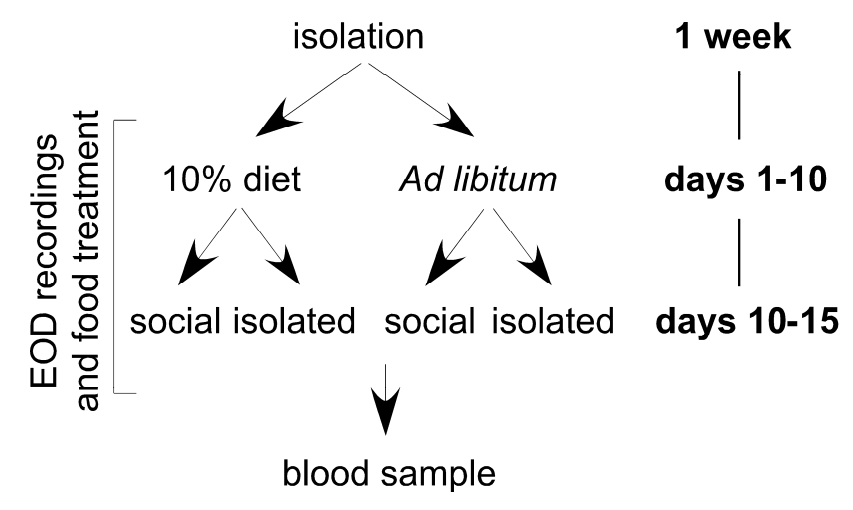

Figure 5.2. Experimental design of Experiment 2: manipulating allostatic load.

Following a week of isolation, the signals of all males were recorded around the clock for 15 days. When EOD recordings started, males were divided in two diet groups: food restriction vs. ad-lib food. After 10 days of diet treatment, half the males in each diet received a social stimulus, resulting in four treatment groups among a $2 \times 2$ design of [food restriction $v s . a d-l i b$ food] and [social isolation $v s$. social challenge]. After five days of food and social treatment, we collected a blood sample from all males. 

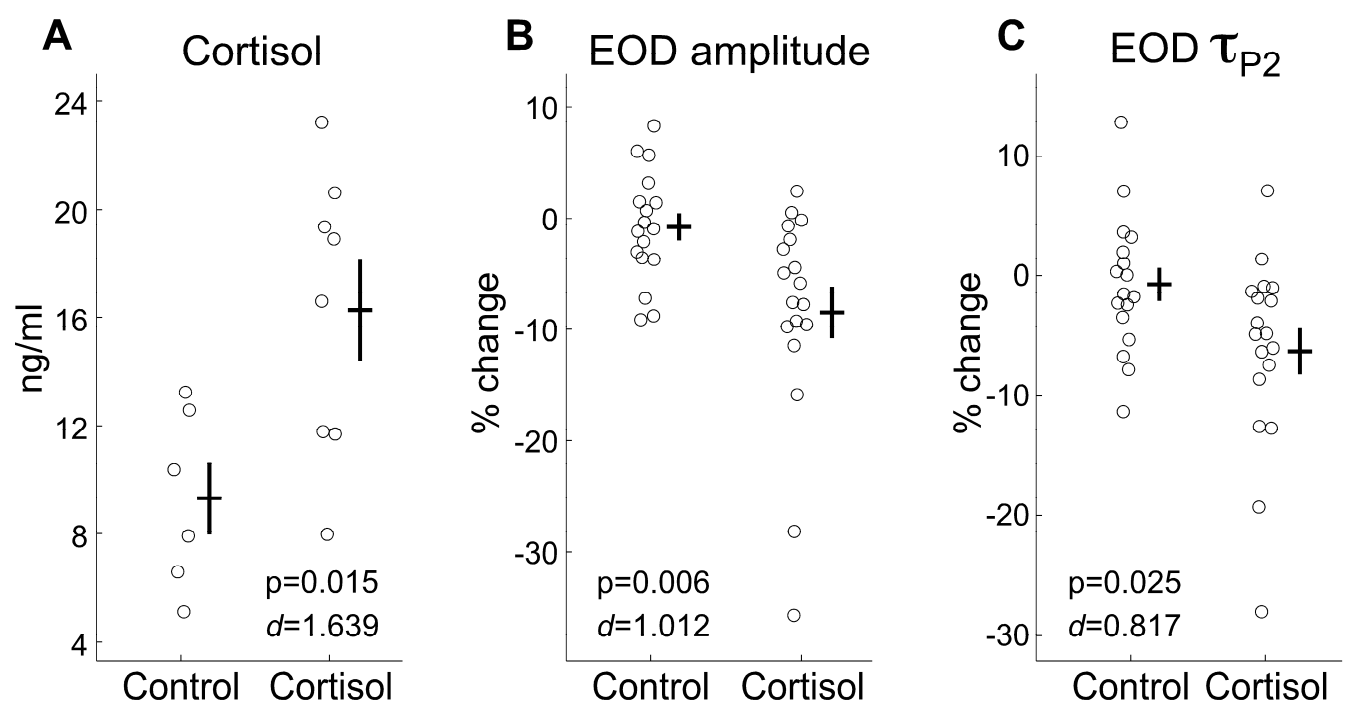

Figure 5.3. Effect of cortisol administration on circulating levels of cortisol and on the EOD waveform. (A) Cortisol administration significantly increased circulating levels of cortisol. (B) Cortisol treatment resulted in a decline in EOD amplitude and (C) on the duration of the EOD's $2^{\text {nd }}$ phase. For each group, vertical bar $=S E$, horizontal bar $=$ mean. Effect sizes are given by Cohen's $d$; p values are also displayed. 

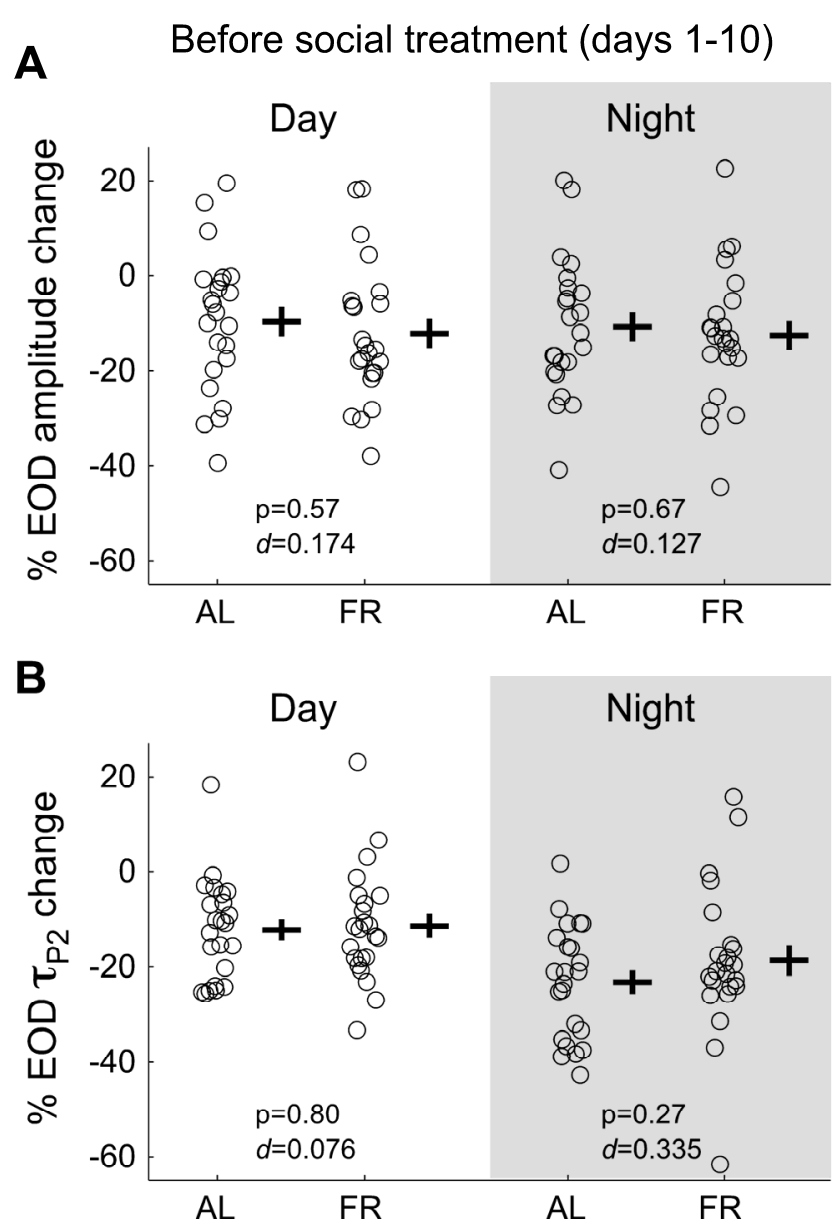

Figure 5.4. Effect of 10 days of diet (FR: food restricted, AL: ad libitum) on the electric organ discharge (EOD). Although most fish decreased both EOD parameters during the first 10 days in the experiment, the change in EOD amplitude (A) and EOD duration (B) were not related to diet treatment. Effect sizes are given by Cohen's $d ; \mathrm{p}$ values are also displayed. Circles depict data points, horizontal lines and vertical lines indicate mean and SEM respectively. 

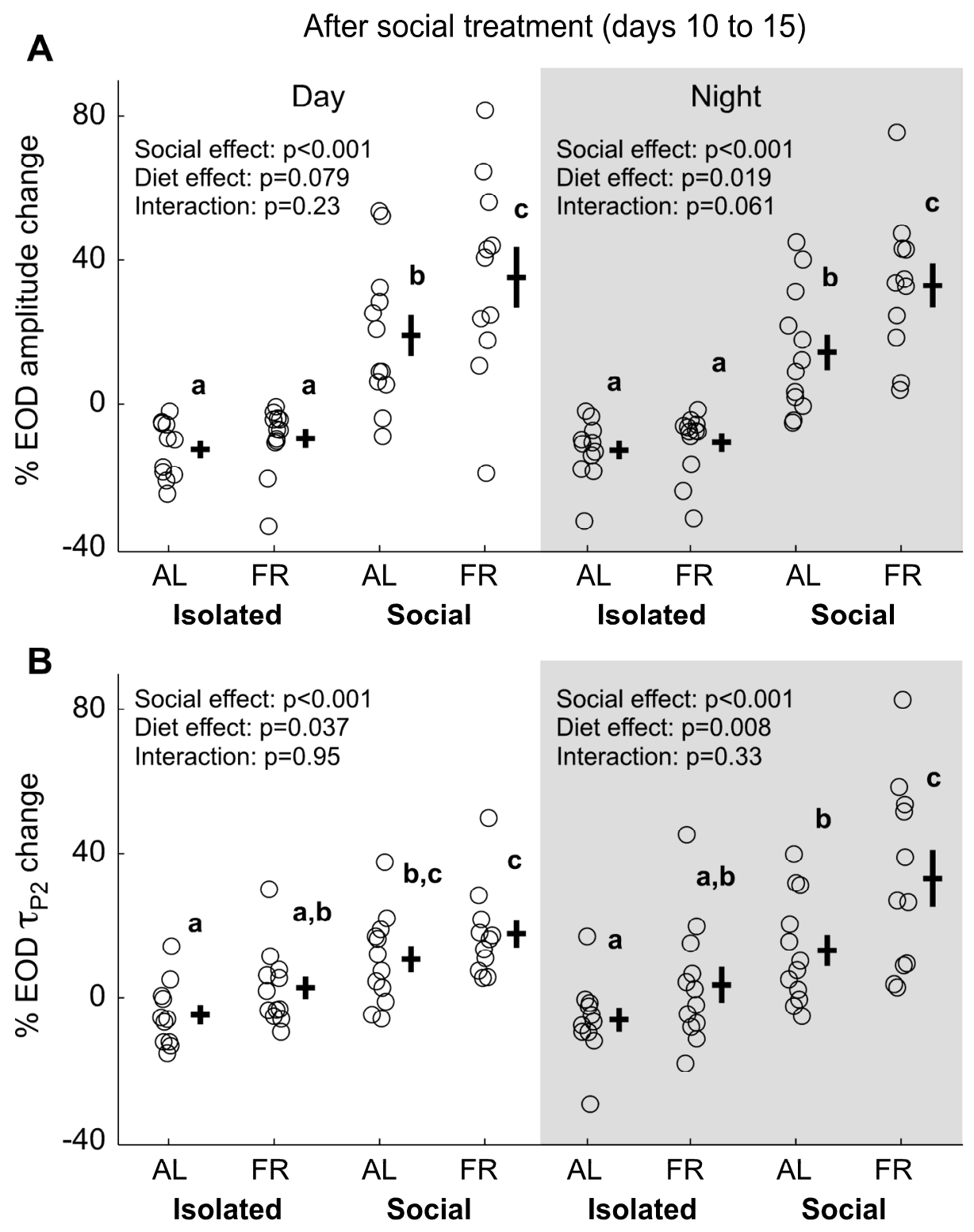

Figure 5.5. Effect of food restriction and social treatment on the electric organ discharge (EOD). Males in both diet treatments (FR: food restricted, AL: ad libitum) increased the amplitude (A) and duration (B) of their EODs in response to social challenge. Changes in the EOD occur for daytime and nighttime recordings. However, males in FR diet increase both the amplitude and the duration of the EOD further than males in AL diet. Lower 
case letters indicate group differences within a plot. Circles indicate data points, horizontal lines indicate treatment means, while vertical lines indicate SEM.
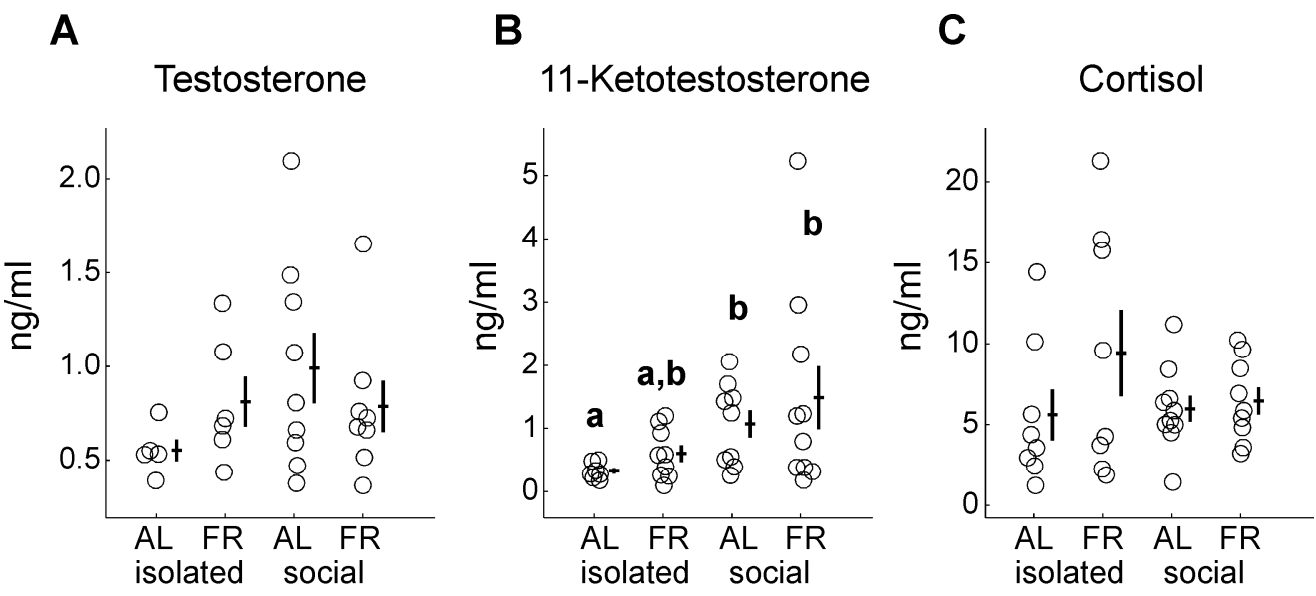

Figure 5.6. Hormone profile for each treatment group in experiment 2. 11ketotestosterone (B) increased with social challenge and increased slightly with food restriction. However, neither testosterone (A) nor cortisol (C) were affected significantly by food or social treatment. Significant differences between treatments, when present, are indicated by lower case letters. Group means and SEM are depicted by horizontal and vertical lines respectively. 

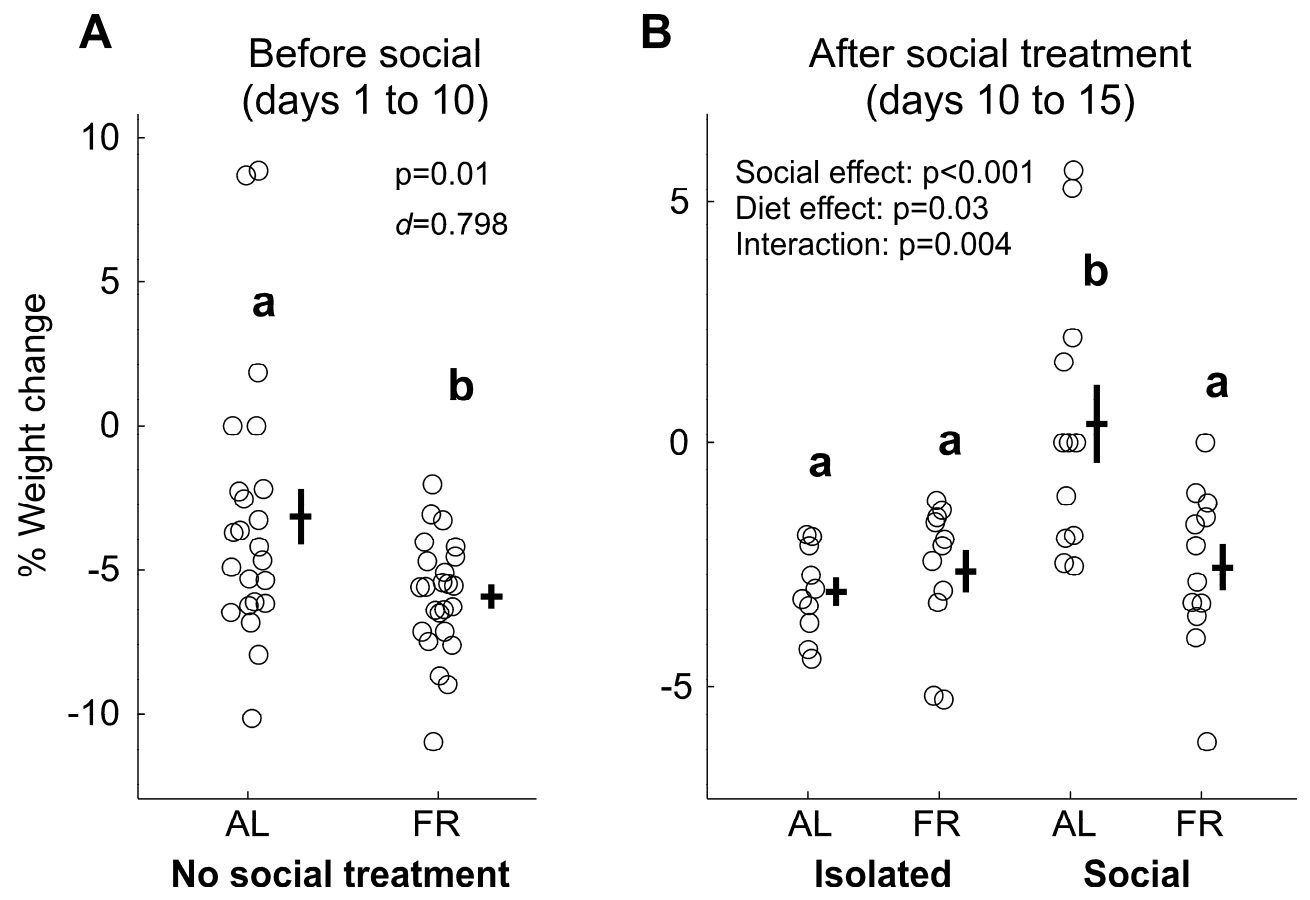

Figure 5.7. Percentage of change in body weight. A) After 10 days of food treatment, the groups in the food restricted (FR) diet lost more weight than fish in the ad libitum (AL) treatment. B) After five days of social interaction, the group with AL food with social companion had lost less weight than the other groups, and some males even gained weight, while no fish in the other treatments gained weight. Effect sizes are given by Cohen's $d$ and $\mathrm{p}$ values are also displayed. Lower case letters indicate significant differences between treatments within a graph. 


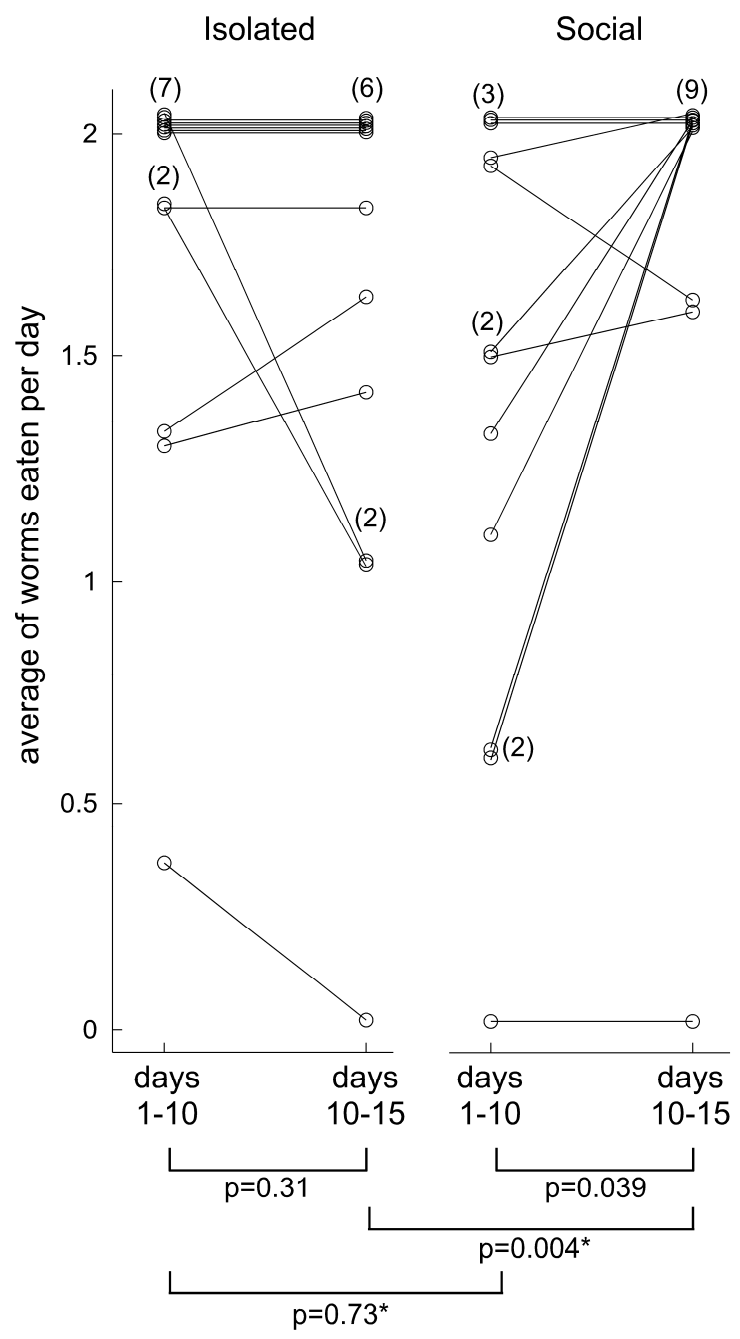

Figure 5.8. Average number of worms eaten daily by fish in the food-restricted treatments before social treatment (days 1 to 10) and after social treatment (days 10 to 15). For the fish that remained in isolation throughout the experiment, their worm consumption did not change, while fish that received a social stimulus significantly changed the number of worms they ate. Moreover, while social and isolated groups ate similar number of worms before social treatment, they differ once the social stimulus was added. Numbers in parenthesis indicate the number of points in the cluster. * Since we tested for differences between social treatment twice (days 1-10 and days 10-15), we used a Bonferroni corrected $\alpha=0.025$. 


\section{CHAPTER 6}

\section{SOCIAL REGULATION OF ELECTRIC SIGNAL PLASTICITY IN MALE BRACHYHYPOPOMUS GAUDERIO}

As submitted to Journal of Comparative Physiology A

June 2012 


\begin{abstract}
Male costly signals are the result of sexual selection acting via male-male competition and/or female mate choice. Dynamic signals allow the adjustment of their display intensity to the costs and benefits of the social context. The social context of changes in the signal provides indirect clues to the traits that are relevant for receivers. The presence of females increases the expression of signals relevant for mate choice, while the presence of competitors promotes the enhancement of signals involved in malemale competition. The electric fish Brachyhypopomus gauderio produces a biphasic electric signal pulse for electrolocation and communication. Male pulses are greater in amplitude and second phase duration than female pulses. Moreover, males further enhance the amplitude and duration of their pulse when the number of males and females increase simultaneously. The amplitude of the pulse conveys information about body size while the duration of the second phase conveys information about reproductive state and possibly aggressive intent. Here I tested the relative effect of female presence and male presence on signal enhancement. I also tested whether the size of the male competitor affected signal enhancement. I found that male presence drives the enhancement in both pulse amplitude and second phase duration. However, signal enhancement was irrespective of the opponent's size. Female presence only had an effect on the duration of the pulse's second phase. I suggest that males cue into information about body size coded by EOD amplitude and aggressiveness coded by pulse duration, while females may be primarily concerned about information on reproductive condition coded by pulse duration.
\end{abstract}




\section{Introduction}

The evolutionary stability of a communication system depends on the quality of the information conveyed by the signaler and the receiver's ability to decode that information (Andersson 1994; Bradbury and Vehrencamp 1998; Searcy and Nowicki 2005). Thus, understanding the function of communication signals requires the study of the signaling system from the sender-perspective and the receiver-perspective (Vehrencamp et al. 2007). The sender-based approach involves the study of the information conveyed by the signal and the context in which those signals are produced (Font and Carazo 2010; Seyfarth et al. 2010). The receiver-based approach involves the study of receiver's response to signals (Vehrencamp et al. 2007). The receiver-perspective has been the subject of multiple studies of signal function in aggression and territoriality, where a receiver is presented with a playback signal and its response is recorded. However, this receiver-based approach has been criticized because of the ambiguity in the interpretation of the receiver's response. For instance, while a threatening signal may result in retreat or weak response from the receiver, a motivated receiver in good condition may instead increase its aggression and responsiveness to the signal. Thus, a receiver-based approach confounds the function of the signal with the motivation and condition of the receiver (Searcy and Nowicki 2005). Therefore, a sender-based approach may provide an alternative or complementary information to the study of signal function (Vehrencamp et al. 2007).

I used a sender-perspective to study the function of the electric signal of the gymnotiform Brachyhypopomus gauderio. The electric fish B. gauderio is an excellent 
model to study the function of the electric signal in communication, the signal is easily quantified, the signal changes accordingly to the social context, and recently I have elucidated the information conveyed by these signals. Male and female B. gauderio produce a biphasic electric organ discharges (EOD) for communication and navigation (Stoddard 2007). The male EOD is greater in amplitude and longer in the duration of the second phase (Hopkins et al. 1990; Franchina and Stoddard 1998). Males further enhance EOD amplitude and second phase duration during the night and when social competition increases (Franchina and Stoddard 1998; Franchina et al. 2001; Salazar and Stoddard 2009). Two main mechanisms modulate the changes in the EOD, each mechanisms is regulated by a different set of hormones and operates at a different timescale (Franchina et al. 2001; Stoddard et al. 2006). The first mechanism results in short-term changes that occur within minutes of social interaction and with the transition from day to night, these changes are regulated by melanocortin hormones a-melanocyte stimulating hormone (aMSH) and adrenocorticotropic hormone (ACTH) (Markham and Stoddard 2005;

Stoddard et al. 2006; Markham et al. 2009). The second mechanism results in long-term changes noticeable after days of social interaction, these long-term changes are regulated primarily by androgens (Hagedorn and Carr 1985; Silva et al. 2002; Stoddard et al. 2006; Allee et al. 2009; Goldina et al. 2011).

The amplitude of the EOD is physically related to body length (Hopkins et al. 1990; Curtis and Stoddard 2003; Gavassa et al. 2012). Body length is related to EOD amplitude in two ways: 1) the EOD is produced by the electric organ, which runs along the fish's length, longer fish have longer electric organs capable of producing EOD's of 
greater amplitude (Hopkins 1999), and 2) a fish generating an EOD is also modeled as a dipole source, voltage output increases with dipole separation, while dipole separation is proportional to body length (Stoddard et al. 1999). Amplitude enhancements further tighten the relationship between body length and EOD amplitude, at the highest EOD amplitude $96 \%$ of the variation in EOD amplitude is explained by body length (Gavassa et al. 2012). However, the EOD is energetically expensive forcing B. gauderio to be very conservative with its EOD enhancement (Salazar and Stoddard 2008; Stoddard and Salazar 2011). Males decrease the amplitude of the EOD during the day and with social isolation (Franchina and Stoddard 1998; Franchina et al. 2001), reducing energetic expenditure but also compromising the quality of the information about body size conveyed by the signal (Gavassa et al. 2012).

On the other hand, the duration of the EOD's second phase conveys information about reproductive condition and androgen levels (Gavassa et al. 2011). The duration of the EOD's second phase is regulated by androgens (Silva et al. 2002; Stoddard et al. 2006; Allee et al. 2009; Goldina et al. 2011); thus, it reflects endogenous levels of the circulating androgens testosterone $(\mathrm{T})$ and 11-ketotestosterone (11-KT). Moreover, androgens link the duration of the EOD's second phase to other traits related to androgen levels such as gonad size in males (Gavassa et al. 2011). It is possible that aggressive motivation, which is commonly regulated by androgens in male vertebrates, is also indicated by EOD duration. Extending the duration of the EOD's second phase is costly in terms of predation, the asymmetry between the two phases turns the EOD more conspicuous to predators (Hanika and Kramer 1999; Stoddard 1999; Hanika and Kramer 
2000). Furthermore, the EOD's second phase duration may also be energetically expensive (Salazar and Stoddard 2008), and potentially physiologically expensive too due to androgen-associated costs in immune function (Wingfield et al. 1990). Once again, the cost of signal enhancement should encourage a very conservative use of the EOD's second phase elongation.

Although I now understand the information conveyed by the signal, and how that information is regulated, I still do not know whether this information is relevant to receivers and whether receivers use this information to modify their behavior. Here I use a signaler-based approach to study what information signalers broadcast when interacting with potential mates, potential competitors or both. I also tested whether the signaler's response depended on the relative competitive ability of the competitor, by presenting a challenger male who was larger, smaller or similar size than the focal male. This signalerbased approach will guide me towards what information is more relevant to a specific type of receiver, and is the beginning in the understanding of the evolutionary forces driving signal enhancement.

\section{Materials and methods}

\section{$\underline{\text { Subjects }}$}

I sampled 18 sexually matured males from our $19^{\text {th }}$ generation captive-reared colony at Florida International University (mean \pm s.d.: total length $=19.1 \pm 2.4 \mathrm{~cm}$, weight $=9.6 \pm 2.4 \mathrm{~g}$ ). Our colony is maintained in outdoor pools covered with water hyacinths (Eichornia crassipes). Water conductivity kept at $70-100 \mu \mathrm{S} \mathrm{cm}^{-1}$. Each male 
was weighed, measured, and individually marked with alphanumeric elastomer tags. Experimental procedures were performed under the guidelines and approval of the Institutional Animal Care and Use Committee of the Florida International University, Miami, FL (protocol 09-012).

\section{$\underline{\text { EOD recordings }}$}

Signal recordings were made by the automated system for recording calibrated EODs from freely swimming fish around the clock called the EOD Machine (Stoddard et al. 2003). The EOD Machine is composed of 12 tanks, each divided into three sections by mesh partitions. The outer segments are connected by an unglazed ceramic tube. The focal fish is placed in either one of the outer segments and the fish seeks shelter inside the ceramic tube, strategically positioned in the center of the tank for accurate EOD recordings. A set of electrodes in the tube detect when the fish is in place and trigger the recordings from a pair of nichrome electrodes located at the ends of the tank. The signal is amplified 500X and low-pass filtered, and instantly analyzed by the EOD Machine's computer. Water conductivity was kept at $100 \pm 5 \mu \mathrm{Sm}^{-1}$ and water temperature at $29 \pm$ $1{ }^{\circ} \mathrm{C}$. I focus here on EOD amplitude, estimated at the voltage difference between the positive and negative peaks of the EOD, and EOD second phase duration $\left(t_{\mathrm{P} 2}\right)$, the time constant of repolarization of the second phase (Fig. 1). When used, social stimuli are placed in the inner segment of the tank, where they can interact electrically with the focal fish but with no physical contact. 


\section{Pharmacological partitioning of signal enhancement mechanisms}

Melanocortins are responsible for the first mechanism for signal enhancement, which consists of short-term and energetically inexpensive changes to the signal (Markham and Stoddard 2005; Stoddard et al. 2006; Stoddard and Markham 2008). The second mechanism is regulated by androgens, which drive long-lasting changes that are likely to increase energetic expenditure (Stoddard et al. 2006; Allee et al. 2009; Goldina et al. 2011; Stoddard and Salazar 2011). In order to separate signal enhancements caused by melanocortin action from androgen driven changes I blocked melanocortin effects using a synthetic cyclic-a-MSH analog (Fig. 2), which either blocks or attenuates melanocortin effects (Markham et al. 2009).

Prior to presenting the stimulus, each male was isolated 8 days to maximally reduce androgen-driven enhancement in the EOD (Franchina et al. 2001; Salazar and Stoddard 2009). Then, males were housed with two mature females for 3 days in the EOD Machine to partially elevate the EOD baselines, leaving room for further increase or decrease (Franchina et al. 2001). On the third day in the EOD Machine, females were removed at 9:00 EST. An hour after removing the females (10:00 EST), experimental males where injected with cyclic-a-MSH (Markham et al. 2009), blocking the short-term effects of melanocortins on the waveform, the remaining expression of EOD parameters is caused by the fish's length and long-term changes promoted by androgens (mechanism 1) (Stoddard et al. 2006; Allee et al. 2009; Goldina et al. 2011; Gavassa et al. 2012). The EOD parameters following the initial cyclic-a-MSH provide the baseline levels for androgen and size driven EOD parameters (Fig. 2). The following afternoon (16:00 EST), 
focal males were presented with a social stimulus, according to treatment and experiment, and allowed to interact electrically but not physically for three nights. On the morning after the third night of social interaction, the social stimuli were removed (9:00 EST) and focal males were injected one last time with cyclic-a-MSH (10:00 EST) to prove the extent of androgen-driven changes in the EOD.

\section{Experiment 1: Effect of stimulus sex}

I randomly assigned each male to one of four treatments: 1) male stimulus, 2) female stimulus, 3) male and female stimuli, or 4) no stimulus (isolation). The day following aMSH injection, the designated stimulus type was introduced to the middle compartment of the focal male. All fish went through all treatments in random order. Females used for stimulus looked gravid, evidenced by swollen abdomens. Males used as stimulus where matched within $1 \mathrm{~cm}$ to the focal fish' total length.

\section{Experiment 2: Effect of stimulus size}

The protocol was the same as in experiment 1, but with different treatments. All males went through each of three treatments in random order: 1) larger male, 2) male of similar size, and 3) smaller male. Like in experiment 1, the size-matched male was within $1 \mathrm{~cm}$ in total body length from the focal male. Larger or smaller stimulus males differed by at least $3 \mathrm{~cm}$ in total body length to the focal male. To minimize the number of study subjects, I run experiments 1 and 2 simultaneously on the same individuals. However, treatment order was completely randomized. The similar size male stimulus treatment was shared by both experiments. 


\section{Analyses}

The difference between the knocked down daytime EOD parameters before social interaction (following the first cyclic-a-MSH injection) and knocked down daytime EOD parameters after three days of social interaction (following the second cyclic-a-MSH injection) indicated the magnitude of long-term changes (mechanism 2, Fig. 2). I estimated the slope of long-term changes and used it as a baseline to calculate short-term changes. The difference between the estimated baseline from mechanism 1 and the nighttime peak on the first night with the social stimulus indicates the magnitude of melanocortin-driven EOD enhancements after initial presentation of the stimulus (initial mechanism 1). The magnitude of melanocortin-driven EOD enhancements was estimated a second time, after three nights of social interaction, calculating the difference between estimated baseline from long-term changes and the nighttime peak EOD parameters on the third night of social interaction (final mechanism 1). These pharmacological manipulations allow me to estimate the relative contribution of short-term (mechanism 1) and long-term (mechanism 2) mechanisms in EOD enhancement during social interactions. Changes in EOD parameters are shown as the percentage of change. For experiment 1 I performed two-way repeated measures ANOVA using a factorial design with two factors (male or female stimulus) and two levels (presence or absence) to test for significant differences among groups. I tested the effect of each stimulus type (male or female) as well as the interaction of simultaneously presenting a male and a female. For experiment 2 I used one-way ANOVA with repeated measures with three treatments: 
larger, similar or smaller stimulus male. Statistical analyses were performed using the MatLab Statistical Toolbox and SPSS v.18 (SPSS, Chicago, IL, U.S.A.).

\section{Results}

\section{Experiment 1: Effect of stimulus sex}

As seen in a previous study (Franchina et al. 2001), I found that EOD amplitude and $t_{\mathrm{P} 2}$ both responded to male presence, while $t_{\mathrm{P} 2}$ was more responsive to female presence than EOD amplitude (Fig. 3). EOD amplitude rapidly increased after adding a stimulus male, reaching a higher nighttime peak in the treatments that included a stimulus male than those with just a female or no stimulus (initial mechanism 1, Table 1, Fig. 3a). After three days of social interaction, the proportional increase in EOD amplitude at nighttime remained significantly higher in the treatments with a male stimulus (final mechanism 1, Table 1, Fig. 3b). Likewise, long-term changes in EOD amplitude were only seen in treatments including a male stimulus (mechanism 2, Table 1, Fig. 3c). Accordingly, the combination of mechanism 1 and 2 in EOD amplitude was also only influenced by male stimulus (Table 1, Fig. 3d).

In contrast to what happened in EOD amplitude, $\mathrm{t}_{\mathrm{P} 2}$ rapidly increased on the first night of social interaction in treatments receiving either male or female stimulus (Table 1, Fig. 3e). However, the female effect on nighttime increase had disappeared by the third night of social interaction and only the male stimulus effect was still evident (Table 1, Fig. 3f). Interestingly, only female presence had an effect on the long-term, mechanism 2, enhancement of $t_{\mathrm{P} 2}$ parameter (Table 1, Fig. 3g). Yet, the total change in $\mathrm{t}_{\mathrm{P} 2}$ (combining short and long-term changes) was only apparent in treatments with a male challenger (Table 1, Fig. 3h). 


\section{Experiment 2: Effect of stimulus size}

Prior studies had suggested that the responsiveness to a challenging male could depend on the relative size of the challenger in respect to the focal male (Franchina et al. 2001; Salazar 2009). Here I systematically tested whether the effect of the stimulus male size had an effect on the response of the focal male. I could not detect an effect of the stimulus male's size on the modulation of neither EOD amplitude nor $t_{\mathrm{P} 2}$ for neither mechanism (Table 2, Fig. 4).

\section{Discussion}

The purpose of this study was to investigate how signalers modify their displays depending on the social context. My assumption was that one can infer the function of specific signal parameters by studying the social contexts that promote the enhancement of those parameters. For instance, if a signal parameter is enhanced in the presence of females but not in the presence of males, then I could infer that the signal parameter is relevant for female choice. On the other hand, a signal parameter that is enhanced in the presence of males but not in the presence of females may be relevant for male-male competition. Alternatively, a signal parameter could have dual function and be present in both contexts.

$\underline{\text { Signal changes accordingly to stimulus sex }}$

Consistent with prior reports, I found that the amplitude of the EOD is more responsive to other male social challengers than to female companion, while EOD

duration responds to the presence of challengers of either sex (Franchina et al. 2001). 
However, in this study I dissected the relative contribution of each EOD enhancement mechanism to the observed changes as opposed to prior studies where just total changes in the signal were analyzed. My approach allowed me to see that long-term changes have opposite sensitivity to sex of the stimulus fish, with EOD amplitude's mechanism 2 responding to male stimulus and EOD duration's mechanism 2 responding to female stimulus only. I also show the relative magnitude of each mechanism for EOD enhancement, with mechanism 1 being almost an order of magnitude greater than mechanism 2.

Unexpectedly, I did not find an effect of female stimulus on EOD amplitude enhancement at any timescale. Female-induced changes in EOD amplitude seem to take longer than male-induced changes, taking up to two weeks to see considerable changes (Franchina et al. 2001). On the other hand, $t_{\mathrm{P} 2}$ significantly increased on the first night of social interaction regardless of the stimulus sex, although the response to male stimulus was larger. The effect of female presence on $t_{\mathrm{p} 2}$ enhancement via mechanism 1 had disappeared by the third night of social interaction. Interestingly, only females had an effect on the longer-lasting enhancement of t $t_{\mathrm{P} 2}$ via mechanism 2. The subtle response of $t_{\mathrm{P} 2}$ to female stimulus sex disappeared once I compared total change. The greater magnitude of $t_{\mathrm{P} 2}$ changes via mechanism 1 masked any variation in mechanism 2 once they were combined.

The magnitude of EOD change for each mechanism are comparable to the changes observed with hormone administration of the respective hormonal modulator, where $t_{\mathrm{P} 2}$ has been found to be more responsive than EOD amplitude. For instance, 
melanocortin injection causes about a $100 \%$ increase in $t_{\mathrm{P} 2}$ within a couple hours of injection, while EOD amplitude has only increased about 25\% (Markham and Stoddard 2005; Markham et al. 2009; Goldina et al. 2011). Likewise, 11-KT administration causes about $80 \%$ increase in nighttime $t_{\mathrm{P} 2}$, while it only causes about a $10 \%$ difference in EOD amplitude compared to sham-implanted fish (Goldina et al. 2011). In figure 4 I plotted t $\mathrm{P2}$ and amplitude together to visualize the relative magnitude in change of each parameter. While mechanism 1 is more prevalent in $t_{\mathrm{P} 2}$ enhancement than amplitude enhancement, the range of mechanism 2 is quite comparable for both EOD parameters.

\section{Effect of stimulus size}

It had been suggested that males increased the amplitude of their EOD more in the presence of males larger than themselves than in the presence of smaller males (Salazar 2009). However, I found that males responded very similarly to male stimulus of various sizes (Table 2, Fig. 4). My finding was unexpected, since body size is the main determinant of the winner of an agonistic encounter between $B$. gauderio males (Salazar 2009; Silva et al. 2010). Small resident males should value their resource more and were expected to invest more in defending it by enhancing the amplitude of their EOD and appear or larger size. Nonetheless, signal enhancement is ultimately constrained by the signaler's body length (Gavassa et al. 2012). Thus, even when males have plenty of motivation to exaggerate their body size by enhancing EOD amplitude, the highest EOD amplitude they can reach is tightly related to the fish's body length (Gavassa et al. 2012). 


\section{$\underline{\text { Assessing signal function }}$}

Signal enhancement is expected to benefit receivers when it improves the assessment of the information coded by the signal or stimulates reproduction (Akre and Ryan 2011). The enhancement of EOD amplitude greatly improves the assessment of body size information since the strength by which EOD amplitude predicts body size increases as EOD amplitude increases (Gavassa et al. 2012). As mentioned above, body size information should be relevant to potential competitors since competitive ability depends on body size (Salazar 2009; Silva et al. 2010). Accordingly, EOD amplitude should increase in the presence of potential competitors as I found here. By enhancing its EOD amplitude, the focal male is facilitating the receiver's assessment of his body size and consequently the assessment of his fighting ability. My findings corroborate the notion that EOD amplitude functions as an initial assessment of fighting ability and potentially avoiding the need to physical contact.

Body size information should be relevant for females too. When given a choice, females prefer longer males (Curtis and Stoddard 2003). However, females would not mate with males who are smaller than a certain threshold (Curtis and Stoddard 2003). Contrarily to what I expected, males did not enhance their EOD amplitude in the presence of females, unless there was another male present. It is possible than given the way female-choice seems to operate in this species, when there is only a female present, males just need to make sure their EOD amplitude is above the acceptable threshold for female choice and there is no further advantage on enhancing their EOD beyond that. However, when another male is present, the female chooses the best out of the two. In the 
later case a male who doesn't increase its EOD amplitude while his competitor enhances his EOD would be at a mating disadvantage, even if his EOD is above the acceptable threshold for females.

The duration of the EOD's second phase is an indicator of reproductive state and possible aggressive motivation (Gavassa et al. 2011). Information about reproductive state should be particularly relevant to females, who may pay a great cost of unfertilized eggs when mating with a male in poor reproductive condition. Alternatively, $\mathrm{t}_{\mathrm{P} 2}$ may be necessary to stimulate females to breed, as suggested for male signals of other species (Akre and Ryan 2011). Accordingly, female presence resulted in an initial rapid increase in $t_{\mathrm{P} 2}$ via mechanism 1, and in a long-term increase via mechanism 2. Males should also pay attention to $t_{\mathrm{P} 2}$ since it may provide information about the aggressive motivation of the signaler. Correspondingly, male presence also stimulated an increase in $t_{\mathrm{P} 2}$ but only via mechanism 1.

\section{Conclusions}

Since androgens regulate mechanism 2 enhancements in EOD amplitude and $t_{\mathrm{p} 2}, \mathrm{I}$ expected to find a simultaneous increase in both parameters. However, I found an increase in EOD amplitude but not $t_{\mathrm{P} 2}$ via mechanism 2 in response to male stimulus, while the opposite was found in response to female stimulus. It is possible that another hormone regulates EOD amplitude enhancements via mechanism 2. For instance, melanocortins may have long-term effects on EOD amplitude in addition to the already known short-term effects (PKS, unpublished). In support to this hypothesis, male presence resulted in greater short-term increases in EOD parameters than female presence 
indicating higher levels of circulating melanocortin levels in males when receiving a male stimulus than a female stimulus.

The plasticity of the EOD could make the information coded by the EOD vulnerable to dishonesty via signal exaggeration. To prevent signal dishonesty with signal exaggeration, the mechanisms that regulate signal plasticity should be implicated in regulating the information coded by the signal. As mentioned earlier, androgens also regulate reproductive condition and likely aggressive motivation linking the long-term changes in the signal to changes in the information coded by the signal (Gavassa et al. 2011). On the other hand, the rapid changes on EOD amplitude orchestrated by melanocortins may help reduce signaling costs when there is low incentive for costly signaling and unmask the signal to show its full potential when competition increases (Stoddard and Markham 2008). Melanocortins have even greater effect on $t_{\mathrm{P} 2}$, it is possible that melanocortins also regulate aggression and therefore their release further strengthens the relationship between the signal and its information (Ducrest et al. 2008). Therefore, the enhancement of EOD parameters improves the assessment of information relevant to the specific type of receiver.

\section{Acknowledgments}

I want to give special thanks to James Roach for his help in data collection. I also thank C. Curtis, A. Goldina, E. Gonzalez, E. Machado, J. Molina, and P. Perez for assistance in the lab. This work was supported by FIU University Graduate School's Dissertation Evidence Acquisition Fellowship and Dissertation Year Fellowship to S.G., and NSF grant IOS 0956603 to P.K.S. 


\section{References}

Akre KL, Ryan MJ (2011) Female túngara frogs elicit more complex mating signals from males. Behav Ecol 22:846-853

Allee SJ, Markham MR, Stoddard PK (2009) Androgens enhance plasticity of an electric communication signal in female knifefish, Brachyhypopomus pinnicaudatus. Horm Behav 56:264-273

Andersson M (1994) Sexual Selection. Princeton University Press, New Jersey

Bradbury JW, Vehrencamp SL (1998) Principles of animal communication. Sinauer Associates, Sunderland, MA

Curtis CC, Stoddard PK (2003) Mate preference in female electric fish, Brachyhypopomus pinnicaudatus. Anim Behav 66:329-336

Ducrest A-L, Keller L, Roulin A (2008) Pleiotropy in the melanocortin system, coloration and behavioural syndromes. Trends Ecol Evol 23:502-510

Font E, Carazo P (2010) Animals in translation: why there is meaning (but probably no message) in animal communication. Anim Behav 80:e1-e6

Franchina CR, Salazar VL, Volmar CH, Stoddard PK (2001) Plasticity of the electric organ discharge waveform of male Brachyhypopomus pinnicaudatus II. Social effects. J Comp Physiol A 187:45-52

Franchina CR, Stoddard PK (1998) Plasticity of the electric organ discharge waveform of the electric fish Brachyhypopomus pinnicaudatus. I. Quantification of day-night changes. J Comp Physiol A 183:759 - 768

Gavassa S, Silva AC, Gonzalez E, Stoddard PK (2012) Signal modulation as a mechanism for handicap disposal. Anim Behav 83:935-944

Gavassa S, Silva AC, Stoddard PK (2011) Tight hormonal phenotypic integration ensures honesty of the electric signal of male and female Brachyhypopomus gauderio. Horm Behav 60:420-426

Goldina A, Gavassa S, Stoddard PK (2011) Testosterone and 11-ketotestosteone have different regulatory effects on electric communication signals of male Brachyhypopomus gauderio. Horm Behav 60:139-147 
Hagedorn M, Carr C (1985) Single electrocytes produce a sexually dimorphic signal in South American electric fish, Hypopomus occidentalis (Gymnotiformes, Hypopomidae). J Comp Physiol A 156:511-523

Hanika S, Kramer B (1999) Electric organ discharges of mormyrid fish as a possible cue for predatory catfish. Naturwissenschaften $86: 286-288$

Hanika S, Kramer B (2000) Electrosensory prey detection in the African sharptooth catfish, Clarias gariepinus (Clariidae), of a weakly electric mormyrid fish, the bulldog (Marcusenius macrolepidotus). Behav Ecol Sociobiol 48:218-228

Hopkins CD (1999) Design features for electric communication. J Exp Biol 202:12171228

Hopkins CD, Comfort NC, Bastian J, Bass AH (1990) Functional analysis of sexual dimorphism in an electric fish, Hypopomus pinnicaudatus, order Gymnotiformes. Brain Behav Evolut 35:350-67

Markham M, Allee S, Goldina A, Stoddard P (2009) Melanocortins regulate the electric waveforms of gymnotiform electric fish. Horm Behav 55:306-313

Markham MR, Stoddard PK (2005) Adrenocorticotropic hormone enhances the masculinity of an electric communication signal by modulating the waveform and timing of action potentials within individual cells. J Neurosci 25:8746-8754

Salazar VK (2009) The effect of male-male competition and its underlying regulatory mechanisms on the electric signal of the gymnotiform fish Brachyhypopomus gauderio. Dissertation, Florida International University

Salazar VL, Stoddard PK (2008) Sex differences in energetic costs explain sexual dimorphism in the circadian rhythm modulation of the electrocommunication signal of the gymnotiform fish Brachyhypopomus pinnicaudatus. J Exp Biol 211:1012-1020

Salazar VL, Stoddard PK (2009) Social competition affect electric signal plasticity and steroid hormone levels in the gymnotiform fish Brachyhypopomus gauderio. Horm Behav 56:399-409

Searcy WA, Nowicki S (2005) The Evolution of Animal Communication: Reliability and Deception in Signaling Systems. Princeton University Press, Princeton, NJ, USA 
Seyfarth RM, Cheney DL, Bergman T, Fischer J, Zuberbuhler K, Hammerschmidt K (2010) The central importance of information in studies of animal communication. Anim Behav 80:3-8

Silva A, Quintana L, Ardanaz JL, Macadar O (2002) Environmental and hormonal influences upon EOD waveform in gymnotiform pulse fish. J Physiology-Paris 96:473-484

Silva A, Zubizarreta L, Costa G (2010) Interspecific differences in agonistic behavior and its serotonergic modulation. In: International Congress of Neuroethology, Salamanca, Spain

Stoddard PK (1999) Predation enhances complexity in the evolution of electric fish signals. Nature 400:254-256

Stoddard PK (2007) Plasticity of the electric organ discharge waveform: contexts, mechanisms, and implications for electrocommunication. In: Ladich F, Collin SP, Moller P, Kapoor BG (eds) Communication in Fishes. Science Publisher, Inc., Enfield, NH, USA, pp 623-646

Stoddard PK, Markham MR (2008) Signal cloaking by electric fish. BioScience 58:415425

Stoddard PK, Markham MR, Salazar VL (2003) Serotonin modulates the electric waveform of the gymnotiform electric fish Brachyhypopomus pinnicaudatus. $\mathrm{J}$ Exp Biol 206:1353-1362

Stoddard PK, Rasnow B, Assad C (1999) Electric organ discharges of the gymnotiform fishes: III. Brachyhypopomus. J Comp Physiol A 184:609-630

Stoddard PK, Salazar VL (2011) Energetic cost of communication. J Exp Biol 214:200205

Stoddard PK, Zakon HH, Markham MR, McAnelly L (2006) Regulation and modulation of electric waveforms in gymnotiform electric fish. J Comp Physiol A 192:613 624

Vehrencamp SL, Hall ML, Bohman ER, Depeine CD, Dalziell AH (2007) Song matching, overlapping, and switching in the banded wren: the sender's perspective. Behav Ecol 18:849-859 
Wingfield J, Hegner RE, Dufty Jr AM, Ball G (1990) The "challenge hypothesis": theoretical implications for patterns of testosterone secretion, mating systems, and breeding strategies. Am Nat 136:829-846

\section{Tables}

\begin{tabular}{|c|c|c|c|c|c|c|c|}
\hline & & \multicolumn{2}{|c|}{ Male effect } & \multicolumn{2}{|c|}{ Female effect } & \multicolumn{2}{|c|}{ Interaction } \\
\hline & & Amplitude & $\tau_{\mathrm{P} 2}$ & Amplitude & $\tau_{\mathrm{P} 2}$ & Amplitude & $\tau_{\mathrm{P} 2}$ \\
\hline \multirow{2}{*}{$\begin{array}{c}\text { Initial } \\
\text { mechanism } 1\end{array}$} & $\mathrm{~F}_{1,12} ; \mathrm{F}_{1,13}$ & 12.08 & 154.76 & 0.003 & 4.69 & 0.026 & 0.04 \\
\hline & $p$ value & 0.005 & $<0.001$ & 0.96 & 0.050 & 0.87 & 0.83 \\
\hline \multirow{2}{*}{$\begin{array}{c}\text { Final } \\
\text { mechanism } 1\end{array}$} & $\mathrm{~F}_{1,11} ; \mathrm{F}_{1,13}$ & 7.57 & 32.92 & 0.37 & 0.06 & 0.01 & 0.02 \\
\hline & $\mathrm{p}$ value & 0.019 & $<0.001$ & 0.55 & 0.80 & 0.91 & 0.89 \\
\hline \multirow{2}{*}{ Mechanism 2} & $\mathrm{~F}_{1,13} ; \mathrm{F}_{1,14}$ & 13.22 & 0.24 & 0.72 & 5.02 & 0.02 & 1.41 \\
\hline & $p$ value & 0.003 & 0.63 & 0.41 & 0.042 & 0.89 & 0.25 \\
\hline \multirow{2}{*}{$\begin{array}{c}\text { Mechanisms } \\
1 \text { and } 2\end{array}$} & $\mathrm{~F}_{1,13} ; \mathrm{F}_{1,13}$ & 21.39 & 14.18 & 0.61 & 1.66 & 0.12 & 0.49 \\
\hline & $\mathrm{p}$ value & 0.001 & 0.002 & 0.43 & 0.22 & 0.73 & 0.54 \\
\hline
\end{tabular}

Table 6.1. Results of repeated measures two-way ANOVA for experiment 1, effect of stimulus sex. Effects with p values below 0.05 are shown in bold. Male presence stimulated an increase in EOD amplitude via mechanisms 1 and 2, but only an increase in $t_{\mathrm{P} 2}$ via mechanism 1. On the other hand, female presence had no effect on EOD amplitude but it increased $t_{\mathrm{P} 2}$ via mechanisms 1 and 2. 


\begin{tabular}{clll} 
& & Amplitude & $\boldsymbol{\tau}_{\mathrm{P} 2}$ \\
\hline $\begin{array}{c}\text { Initial } \\
\text { mechanism 1 }\end{array}$ & $\mathrm{F}_{2,13} ; \mathrm{F}_{2,14}$ & 0.41 & 0.067 \\
& $\mathrm{p}$ value & 0.67 & 0.93 \\
$\begin{array}{c}\text { Final } \\
\text { mechanism 1 }\end{array}$ & $\mathrm{F}_{2,13} ; \mathrm{F}_{2,11}$ & 0.25 & 1.15 \\
& $\mathrm{p}$ value & 0.78 & 0.35 \\
Mechanism 2 & $\mathrm{F}_{2,14} ; \mathrm{F}_{2,14}$ & 2.03 & 1.09 \\
& $\mathrm{p}$ value & 0.17 & 0.36 \\
Mechanisms & $\mathrm{F}_{2,13} ; \mathrm{F}_{2,11}$ & 0.27 & 0.193 \\
1 and 2 & $\mathrm{p}$ value & 0.77 & 0.83 \\
\hline
\end{tabular}

Table 6.2. Results of repeated measures one-way ANOVA for experiment 2, effect of stimulus size. There were no significant differences in the response of the focal male to stimulus males of different sizes for neither mechanism of signal enhancement. 


\section{Figures}

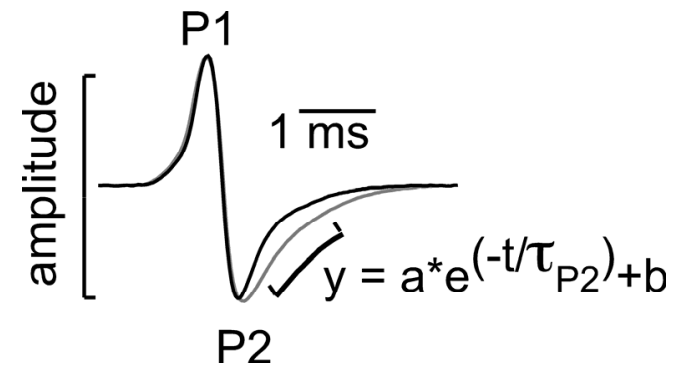

Figure 6.1. The electric organ discharge (EOD) of Brahyhypopomus gauderio. I analyzed total EOD amplitude, measured as the distance from peak-to-peak between the positive and negative phase of the EOD. I estimated the duration of the $2^{\text {nd }}$ phase of the EOD using the parameter $t_{\mathrm{P} 2}$, the time constant of the repolarization of the EOD's second phase. The gray trace shows an EOD with enhanced $t_{\mathrm{P} 2}$ parameter.

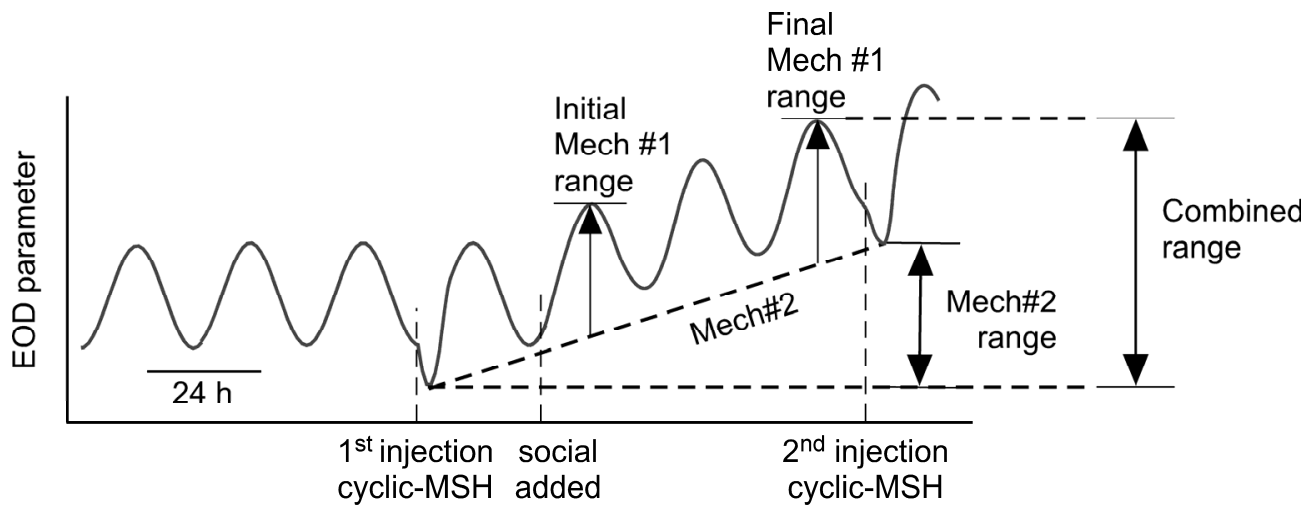

Figure 6.2. Dissecting signal enhancement mechanisms. This diagram shows a representation of the data collected for each EOD parameter throughout the seven days each fish spent in the EOD Machine on a given experimental round. Cyclic-MSH was administered twice in order to separate short-term changes (via mechanism 1) from long- 
term changes (via mechanism 2) in the EOD. The social stimulus was added the day after the first cyclic-MSH injection.
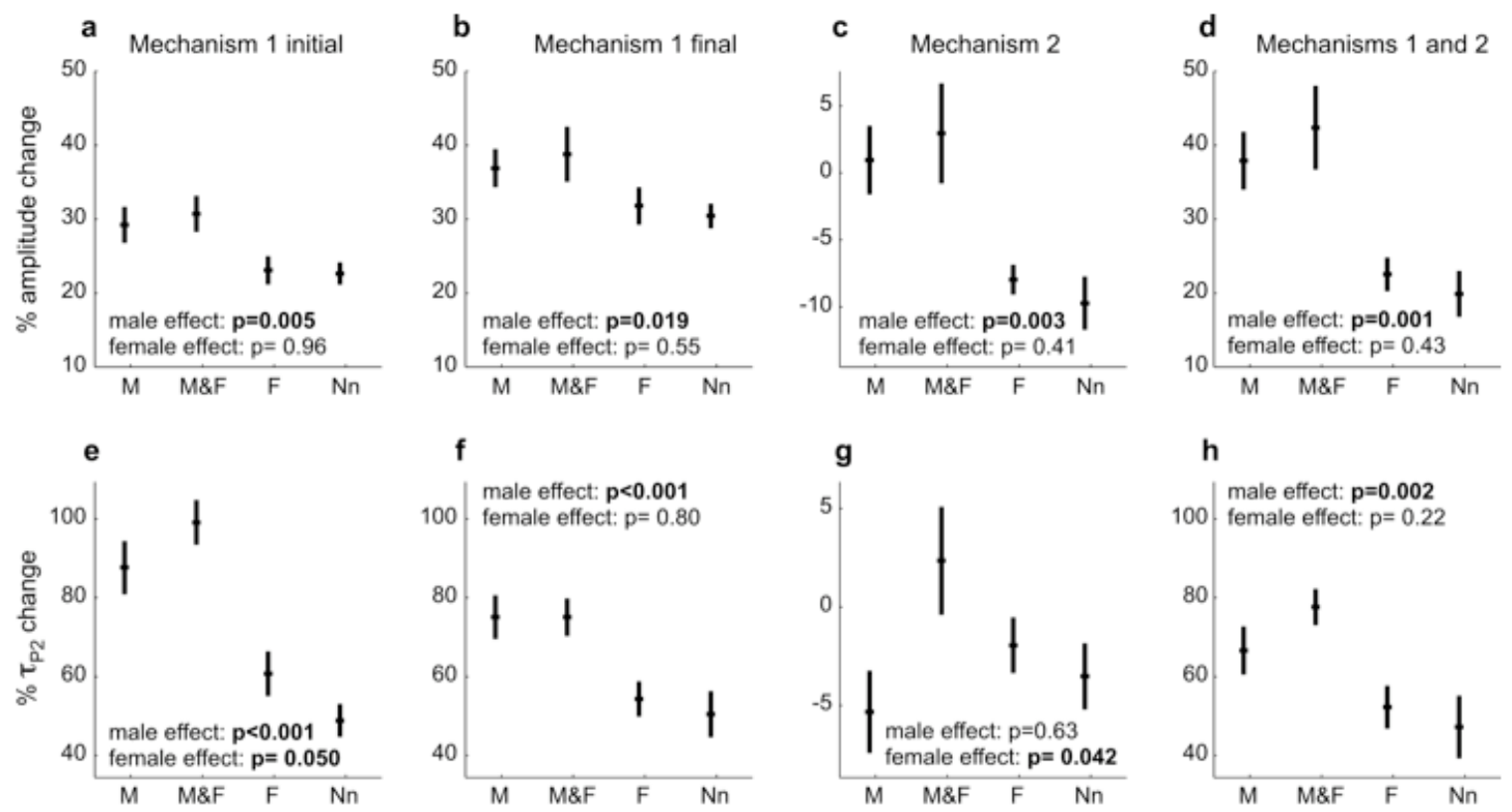

Figure 6.3. Percentage change in EOD amplitude and $t_{\mathrm{P} 2}$ at the two timescales measured for experiment 1: Effect of stimulus sex. Horizontal lines depict mean values and vertical lines depict SEM for each of the four treatments (M: male stimulus, M\&F: male and female stimuli, F: female stimulus, Nn: no stimulus). The p-values for male and female effects resulting from two-way ANOVAs (Table 1) are shown. 

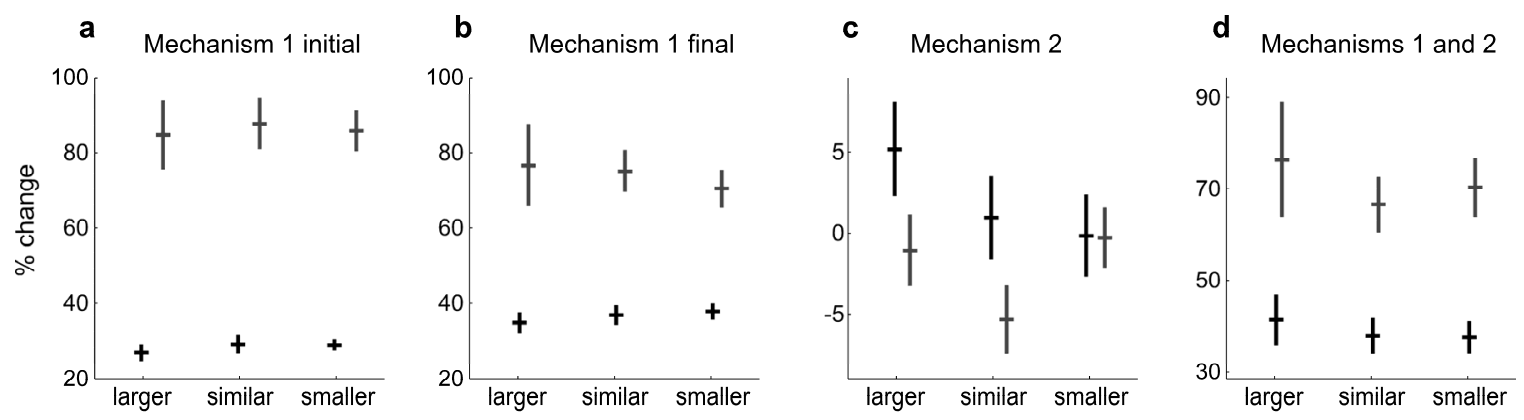

Figure 6.4. Percentage change in EOD amplitude (black) and t $t_{\mathrm{P} 2}$ (gray) for experiment 1: Effect of male stimulus size. Horizontal lines depict mean values and vertical lines depict SEM for each of the three treatments: a stimulus male (i) larger, (ii) similar or (iii) smaller than the focal male. Note that the range of mechanism 1 change in $t_{\mathrm{P} 2}$ is more than twice as high as the range of amplitude change. There was no significant effect of the size of the stimulus on EOD enhancement (see Table 2). 
CHAPTER 7

CONCLUSIONS 
The goals of my dissertation research were to i) estimate the reliability of information conveyed by signals of electric fish, ii) study how the quality of the information changes when the signal changes, and iii) explore the effect of the social and physical environment on signal plasticity.

Is signal modulation decreasing the reliability of the EOD an honest indicator of body $\underline{\text { size? }}$

Despite the good understanding of the neuroendocrine mechanisms behind signal modulation, the effects of signal plasticity on the honesty of the signal were unknown. Salazar \& Stoddard (2008) had proposed that signal enhancement could provide information about the body condition of the signaler. An alternative hypothesis was that EOD modulation would be used for dishonest communication purposes, to exaggerate the signaler's size (Stoddard, NSF grant 2009). The former hypothesis was supported in a study by Salazar \& Stoddard (2008) that found a correlation between the residuals of EOD amplitude (obtained from a linear regression between body length and EOD amplitude) and the residuals of body weight (obtained from a linear regression between body length and body weight). However, residual analyses are very sensitive to the shape of the relationships between the variables (Jakob et al., 1996; Green, 2001). The limited sample size from lab studies makes it impossible to test the adequate fit of the linear regression. All prior studies had assumed a linear relationship between EOD amplitude and body length (Hopkins et al., 1990; Curtis and Stoddard, 2003; Salazar and Stoddard, 2008). My extensive field data set allowed me to test the fit of a linear regression between both: 1) EOD amplitude and body length, and 2) body length and body weight. 
In Chapter 2, I show that neither one of these relationships is linear, EOD amplitude and body weight both increase exponentially with body length. Therefore, the prior thought connection between body condition and EOD amplitude was just an effect of the lack of fit of both linear regressions. Moreover, I found that in the field body length determines up to $96 \%$ of the variation in EOD amplitude, leaving very little room for another factor to explain the remaining variation in EOD amplitude. In fact, the $4 \%$ of the variation left unexplained corresponds to my measuring error of $0.2 \mathrm{~cm}$ for body length measurements.

I also provide a possible explanation for the non-linear relationships. I suggest that the exponential relationship between body length and EOD amplitude comes from the combination of two linear effects of body size on EOD amplitude: longer fish have longer electric organs, with more electrocytes connected in series (Hopkins, 1999), from which we expected a linear relationship between length and EOD amplitude. However, a fish generating an electric discharge resembles a dipole, longer fish have a greater dipole separation (Stoddard et al., 1999), which on its own would result in a linear relationship between body length and EOD amplitude. When both effects, more electrocytes in series and greater dipole separation, are combined, they explain the exponential relationship between body length and EOD amplitude.

Despite my improvement in the model of signal production, my field data revealed a great degree of natural variation in the tightness of the relationship between body length and EOD amplitude. Based on the dishonest communication hypothesis, one would expect that the reliability of the relationship between EOD amplitude and body length would decrease as males enhance their EOD amplitude. However, I found the 
opposite. The tightest relationship between EOD amplitude and body length occurs at the highest population densities when EOD amplitude is the highest, precisely when one would expect the greatest benefits from bluffing. I replicated the field observations in a controlled lab experiment and again showed the relationship between EOD amplitude and body length tightening with competition intensity.

The exponential relationship between body length and EOD amplitude also contributes to reinforcing the honesty of the EOD, since for a male fish to effectively exaggerate his size; it would have to greatly increase the amplitude of its EOD. The expected EOD amplitude doubles with every $3.3 \mathrm{~cm}$ difference in length. For example, for a $15 \mathrm{~cm}$ male to appear to be $18 \mathrm{~cm}$ long, he would have to double the amplitude of his EOD, an energetically expensive and perhaps physiologically unattainable undertaking.

Given that the EOD is very sensitive to hormonal manipulation, can we predict the hormonal profile of a signaler from its EOD?

The hormonal drivers of EOD plasticity have been identified as androgen driving long-term changes (Silva et al., 2002; Stoddard et al., 2006; Allee et al., 2009; Goldina et al., 2011), and peptide hormones driving mid-term changes, with serotonin acting centrally (Stoddard et al., 2003; Allee et al., 2008) and melanocortins acting directly on the electrocyte (Markham and Stoddard, 2005; Stoddard and Markham, 2008; Markham et al., 2009). However, a prior lab study failed to correlate endogenous circulating androgen levels and EOD parameters (Salazar and Stoddard, 2009). 
Sex steroids coordinate broad suites of physiological and behavioural traits necessary for reproduction (Wingfield et al., 1990). Of these, androgens in particular regulate the expression of signals relevant for sexual communication by changing structures and activity in neural circuits (Moore et al., 2005; Ball et al., 2008; Bass, 2008; Godwin, 2010). Nonetheless, communication signals that are regulated by sex steroids rarely reflect sex steroid levels at the time the signals are produced, which makes it even less likely that signals can accurately reflect other behaviourally relevant phenotypic traits regulated by sex steroids. Differences in tissue sensitivity, in receptor density or binding affinity, in the time scale of the response to hormone levels, the plasticity of the signal, and the intervention of other hormonal regulators can mask or impair phenotypic integration and ultimately compromise the reliability of the signal (Adkins-Regan, 2008; Ball et al., 2008; Kempenaers et al., 2008; Karubian et al., 2011). Despite these theoretical limitations, in Chapter 3, I found a strong relationship between endogenous androgen levels (testosterone and 11-ketotestosterone) and the duration of the EOD's second phase. Moreover, I found that androgens also linked the duration of the EOD's second phase to other androgen-mediated traits such as gonad size in males and estrogen (which derives from the aromatization of testosterone) in females. Once again, the large sample size I was able to obtain in the field and the almost simultaneous EOD recording and hormone sampling made this possible. On one hand, the variation in EOD parameters and hormone levels was much greater in the field that what we have observed in the lab (see Chapter 4). On the other hand, the large sample size from the field provided me with greater statistical power to find these relationships between hormone levels, signal parameters and other phenotypic traits. 
Interestingly, I found that gonad size can be predicted from the duration of the second phase of the EOD in males but not in females, although it predicted estradiol levels in females. Males may not require information about the female's gonad size, since females that engage in reproductive behaviors will be those that are ready to spawn (Stoddard, unpublished). Moreover, females may pay a large cost of unfertilized eggs if they mate with a male that will not provide enough sperm. Females may also be interested in mating with males with large testes to ensure their sons will have large testes too. Thus, we expect females to pay close attention to the reproductive condition of the male. Additionally, information about circulating androgen levels should also be relevant to same-sex receivers to assess the fighting motivational state of the signaler. Androgen treatment makes females, and probably males too, more aggressive. Females are more likely to attack and bite other females when implanted with androgens (Allee et al., 2009; Perez et al., unpublished). Consequently, conspecifics can gather valuable information from the EOD; females benefit by inferring the reproductive state of males, while both sexes benefit from inferring the motivational state of same-sex signalers.

Is the regulation of EOD plasticity in females similar to that of males?

Females increase the amplitude and duration of their EODs at night, albeit to a lesser extent than males (Franchina and Stoddard, 1998; Stoddard et al., 2007). Signal plasticity among females was hypothesized to save energetic cost during the day (Stoddard et al., 2007; Salazar and Stoddard, 2008) but no social functions were considered. In Chapter 4, I found that female signals also respond to changes in the social environment in a similar way as males do (Salazar and Stoddard, 2009). In general, 
females increase the amplitude of the EOD in response to increases in population density, while the duration of the EOD's second phase increases in response to a greater ratio of females to males. However in the lab, EOD amplitude and second phase duration both increased with population density.

A prior study on female $B$. gauderio found that androgens increase the responsiveness of the EOD to social and hormonal challenges (Allee et al., 2009). I also found a correlation between androgen levels, EOD duration and intrasexual competition in the field. However, I was unable to replicate this finding in the lab. Nonetheless, I did find an increase in EOD duration, which is regulated by androgens (Silva et al., 2002; Allee et al., 2009; Pouso et al., 2010; Goldina et al., 2011). Enhancements of EOD duration are mediated by androgens in conjunction with melanocortins (Allee et al., 2009; Goldina et al., 2011), and are particularly stimulated by intrasexual competition. Males enhance EOD duration more in the presence of other males than in the presence of females (Franchina et al., 2001; Chapter 6), while androgen-treated females enhance EOD duration more in the presence of females than with males (Allee et al., 2009). Moreover, In Chapter 3 I found a strong relationship between EOD duration and androgen levels. Peripherally acting melanocortins (adrenocorticotropic hormone (ACTH) and alpha melanocyte stimulating hormone $(\alpha-\mathrm{MSH}))$ act directly on the electric organ to increase EOD duration (Markham and Stoddard, 2005; Stoddard et al., 2006; Stoddard, 2007; Markham et al., 2009). Furthermore, androgens greatly potentiate the effect of melanocortin administration on EOD duration (Allee et al., 2009; Goldina et al., 2011). Therefore, I suspect that the increase in female's EOD duration seen in the lab 
experiments presented in Chapter 4 correspond to a small increase in androgens (not statistically significant) that nonetheless increased sensitivity to endogenous melanocortins, resulting in a much greater increase in EOD duration when females outnumbered males than when the sex-ratio was unity.

\section{Communication function of EOD parameters}

I propose that the parameters of the EOD have relatively similar functions in male and female communication. In females, the duration of the EOD's second phase serves as a signal during intrasexual competition while EOD amplitude may function as a signal for intraspecific competition. In males, the duration of the EOD's second phase serves for mate attraction and intrasexual competition while EOD amplitude functions as a signal of resource holding potential (RHP) during intrasexual competition.

In Chapter 4 I show that females increase the duration of their EOD's second phase when the adult sex ratio is female-biased, corresponding to an increase in intrasexual competition. I observed the same phenomenon in the field; at the beginning of the breeding season when sexually matured males were scarce and females had the highest motivation to mate and compete to mate with the best male available. Moreover, EOD duration reflects androgen levels (Chapter 3), which in turn regulate female-female aggression (Allee et al., 2009; P. Perez et al. unpublished).

The duration of the EOD's second phase may have a similar role in male-male competition as seen in female-female competition. In Chapter 6 I found that males increased the duration of their EODs in response to male challengers. Since the duration 
of the EOD's second phase predicts androgen levels and aggression providing valuable information to individuals of the same sex, it makes sense that the EOD's second phase is most responsive to changes in intrasexual competition. Likewise, the duration of the EOD's second phase also predicts testis size; this information should be relevant to females. As mentioned before, females in particular should pay attention to the male's reproductive condition. As expected, in Chapter 6 I found that males also increase the duration of their EOD in the presence of females.

In Chapter 4 I show that EOD amplitude increased in females in response to an increase in population density in the lab and in the field. The increased in population density may either increase competition for resources necessary for both sexes such as food or shelter. Alternatively, an increase in population density may increase background noise and promote an increase in EOD amplitude in order to be noticed out of the crowd, or in order to improve the electrolocation function of the signal.

Males show a similar use of EOD amplitude, increasing with population density as shown in Chapter 2. However in Chapter 6, I show that males increase EOD amplitude in response to a male challenger but not to a female challenger. It seems that in males EOD amplitude functions primarily for male-male competition. EOD amplitude enhancement improves the assessment of the information about size conveyed by the signal (Chapter 2). Since body size is the main determinant of RHP in this species (Salazar, 2009; Silva et al., 2010), amplitude enhancements will help determine RHP without engaging in costly physical contests. Females should also be interested in mating with large males. However, this preference in females can only be exercised when there 
are multiple males to choose from. Thus, EOD amplitude enhancements will be necessary to impress females only when there is at least another male present.

Is reproductive signaling favored over energy savings, as expected from a semelparous species? Alternatively, is signal plasticity used to reduce costs when energy is limited?

From its semelparous life-history, B. gauderio is expected to resist stress and continue to reproduce even when its glucocorticoids levels, primarily cortisol, soar. A previous study in the lab had shown an increase in cortisol levels with social competition, and, interestingly, cortisol was tightly correlated to EOD amplitude $\left(\mathrm{R}^{2}=0.90, \mathrm{p}=0.004\right.$; Salazar and Stoddard, 2009). The concurrent increase in cortisol levels with signal enhancement plus the relationship between cortisol and signal amplitude suggests that the electric signal of $B$. gauderio is resistant to cortisol inhibition, and raises the possibility that cortisol positively regulates EOD amplitude.

Cortisol has been shown to regulate courtship displays in other fish, including electric fish. For instance, cortisol induces courtship vocalization in type I (singing morph) males of the plainfin midshipman (Porichthys notatus) (Remage-Healey and Bass, 2004; Remage-Healey and Bass, 2007). In the brown ghost knifefish (Apteronotus leptorhynchus), a gymnotiform electric fish, social interactions raise cortisol levels and increase the production of electric chirp signals, rate modulations produced during aggressive interactions (Dunlap, 2002). The effects of social interactions on chirping in brown ghosts could be replicated by two weeks of cortisol administration (Dunlap, 2002). Cortisol has been proposed to enhance signal production indirectly by increasing cell 
addition and radial glial fiber density in the diencephalic periventricular zone of brown ghost (Dunlap et al., 2006; Dunlap et al., 2008).

Contrary to those predictions, in Chapter 5 I found that cortisol administration decreases EOD amplitude. Nevertheless, I still found a positive relationship between cortisol levels and EOD amplitude in socially challenged fish (Fig. 1). Since cortisol administration not only failed to enhance EOD waveform but actually decreased the EOD waveform, I do not think the relationship between cortisol and EOD amplitude is causal. Instead, it may have resulted from pleiotropic action of melanocortins (ACTH, adrenocorticotropic hormone, and $\alpha-\mathrm{MSH}$, alpha-melanocyte stimulating hormone), which in addition to promoting the release of cortisol, regulate EOD amplitude (Markham et al., 2009) and synergize with androgens to produce even greater effects in EOD amplitude (Allee et al., 2009; Goldina et al., 2011). As androgens increase in the social treatments, the effect that melanocortins have on the amplitude of the EOD increases as well, resulting in a better correlation between cortisol and EOD amplitude.

In Chapter $5 \mathrm{I}$ show that $B$. gauderio is not resistant to cortisol inhibition as commonly found in other semelparous species, and the prior correlations in our lab had suggested. Therefore, I propose that because $B$. gauderio has a breeding season relatively long compared to most semelparous species, $B$. gauderio should be more conservative in its energetic allocation in order to survive most of the breeding season. In support of my hypothesis, in Chapter 5 I found that when energy is limited and competition increases, B. gauderio increases foraging intensity in order to fuel costly signaling before compromising its energetic reserves. However, when there is no food available and 
competition intensifies, B. gauderio increases reproductive output while it burns through its energetic reserves as a typical semelparous species. This flexible strategy for energy management maximizes survival throughout the breeding season without compromising reproductive performance.

The intermediate levels of competition simulated in Chapter 5 have no effect rising cortisol levels. However, higher competition intensity results in an increase in cortisol (Salazar and Stoddard, 2009). The hormonal pattern resembles what predicted under the Energetics-Hormone Vocalization (EHV) model (Emerson, 2001). According to the EHV model, signaling output should decrease as energy reserves decline. The EHV model proposes that energetically expensive signaling would elevate circulating androgens, as predicted by the Challenge Hypothesis (Wingfield et al., 1990), but it would also elevate glucocorticoids to fuel signaling. Prolonged signaling will decrease energetic stores until glucocorticoids rise to a level at which they inhibit androgen release. The resultant drop in circulating androgen then causes signaling output to decline (Emerson, 2001; Moore and Jessop, 2003). However, I do not think the EHV entirely applies to B. gauderio. Although exogenous cortisol administration decreased the EOD, endogenous cortisol levels that parallel the doses administered have been reported concomitantly with EOD enhancements (Salazar and Stoddard, 2009). I predict that $B$. gauderio prevents cortisol release during intermediate levels of social competition, but when competition intensifies and cortisol levels do rise, B. gauderio must have an additional mechanism to prevent cortisol from inhibiting signaling. Such mechanism, I propose, could be a compensatory increase in androgen levels. Compensatory androgen 
release has been proposed as a mechanism for stress resistance in other species, such as male olive baboons and male Arctic ground squirrels (Sapolsky, 1982; Boonstra et al., 2001; Wingfield and Sapolsky, 2003). Although social stimulation increases androgen levels in all socially stimulated fish in Chapter 5, the group with social competition and food limitation showed the greatest increase in androgen levels.

I conclude that $B$. gauderio has a flexible stress resistance strategy that changes with food availability and urgency to breed. Social stimulation and food limitation both trigger an investment in reproductive signaling, when possible B. gauderio increases foraging to fuel signaling, when no food is available, instead of compromising signaling, B. gauderio increases signaling output and turns to body reserves to fuel signaling.

What have we learned and where to go from there

My dissertation research shows that information about body size, reproductive state and aggressive motivation is conveyed by the signal. I also show that the quality of the information improves instead of degrading when males boost their EOD parameters. Although I explored the function of each EOD parameter in communication, my approach was rather indirect using a sender-perspective to infer signal parameters that could be of interest to receivers. I did not show whether potential mates and potential competitors use the information conveyed by the signal to make behavioral decisions. Future studies are needed to show whether receivers can assess the information conveyed by the signal and what degree of discrimination receivers show for multiple signals. 
This dissertation corroborates a hypothesis previously proposed that in males EOD amplitude tracks the degree of social competition while EOD duration tracks intrasexual competition (Salazar and Stoddard, 2009). Accordingly, I found that EOD amplitude responds to changes in population density in males. Interestingly females also respond to changes in population density by increasing EOD amplitude. On the other hand, EOD duration in females changes with intrasexual competition. Therefore I conclude that in females, EOD amplitude tracks overall competition, but EOD duration is specific to intra-sexual competition. The latter is particularly relevant since EOD duration indicates androgen levels, and androgens increase the likelihood of aggression in females (P. Perez et al., unpublished). Nonetheless, it would also be important to test the fitness consequences of elevated androgen levels in females.

Finally, I found that the social signals of $B$. gauderio do not resist the negative regulation by cortisol as positive correlations had suggested. However, the signals of $B$. gauderio do resist inhibition under the degree of energetic stress simulated in Chapter 5. It would be interesting to test $B$. gauderio stress tolerance over a broader range of stressors. For instance, extend the period of food restriction from 2 weeks (Chapter 5) to a few weeks and increase the intensity of competition. It is also possible that stress tolerance changes with time into the breeding season, with males becoming more tolerant as the breeding season progresses. It would be interesting to test stress tolerance in males in the field at different points into the breeding season. Although I suggest that compensatory androgen release could work as a stress-resistance mechanism, this 
hypothesis still needs to be tested. Moreover, other stress-resistance mechanism may

exist for more intense stressors.

\section{References}

Adkins-Regan, E., 2008. Review. Do hormonal control systems produce evolutionary inertia? Philos. Trans. R. Soc. Lond. B. 363, 1599-609.

Allee, S. J., Markham, M. R., Salazar, V. L., Stoddard, P. K., 2008. Opposing actions of 5HT1A and 5HT2-like serotonin receptors on modulations of the electric signal waveform in the electric fish Brachyhypopomus pinnicaudatus. Horm. Behav. 53, 481-488.

Allee, S. J., Markham, M. R., Stoddard, P. K., 2009. Androgens enhance plasticity of an electric communication signal in female knifefish, Brachyhypopomus pinnicaudatus. Horm. Behav. 56, 264-273.

Ball, G. F., Tlemçani, O., Balthazart, J., 2008. Individual variation and the endocrine regulation of behaviour and physiology in birds: a cellular/molecular perspective. Philos. Trans. R. Soc. Lond. B. 363, 1699-1710.

Bass, A. H., 2008. Steroid-dependent plasticity of vocal motor systems: Novel insights from teleost fish. Brain Res. Rev. 57, 299-308.

Boonstra, R., Hubbs, A. H., Lacey, E. A., McColl, C. J., 2001. Seasonal changes in glucocorticoid and testosterone concentrations in free-living arctic ground squirrels from the boreal forest of the Yukon. Can J Zool. 79, 49-58.

Curtis, C. C., Stoddard, P. K., 2003. Mate preference in female electric fish, Brachyhypopomus pinnicaudatus. Anim. Behav. 66, 329-336.

Dunlap, K. D., 2002. Social interactions and cortisol treatment increase the production of aggressive electrocommunication signals in male electric fish, Apteronotus leptorhynchus. Horm. Behav. 42, 97-108.

Dunlap, K. D., Castellano, J. F., Prendaj, E., 2006. Social interaction and cortisol treatment increase cell addition and radial glia fiber density in the diencephalic periventricular zone of adult electric fish, Apteronotus leptorhynchus. Horm. Behav. 50, 10-17. 
Dunlap, K. D., McCarthy, E. A., Jashari, D., 2008. Electrocommunication signals alone are sufficient to increase neurogenesis in the brain of adult electric fish, Apteronotus leptorhynchus. Developmental Neurobiology. 68, 1420-1428.

Emerson, S. B., 2001, Male advertisement calls: behavioral variation and physiological processes. In: M. J. Ryan, (Ed.), Anuran Communication. Smithsonian Institution Press, Washington, DC, pp. 36-44.

Franchina, C. R., Salazar, V. L., Volmar, C. H., Stoddard, P. K., 2001. Plasticity of the electric organ discharge waveform of male Brachyhypopomus pinnicaudatus II. Social effects. J. Comp. Physiol. A. 187, 45-52.

Franchina, C. R., Stoddard, P. K., 1998. Plasticity of the electric organ discharge waveform of the electric fish Brachyhypopomus pinnicaudatus. I. Quantification of day-night changes. J. Comp. Physiol. A. 183, 759 - 768.

Godwin, J., 2010. Neuroendocrinology of sexual plasticity in teleost fishes. Front. Neuroendocrin. 31, 203-16.

Goldina, A., Gavassa, S., Stoddard, P. K., 2011. Testosterone and 11-ketotestosteone have different regulatory effects on electric communication signals of male Brachyhypopomus gauderio. Horm. Behav. 60, 139-147.

Green, A. J., 2001. Mass/length residuals: Measures of body condition or generators of spurious results? Ecology. 82, 1473-1483.

Hopkins, C. D., 1999. Design features for electric communication. J. Exp. Biol. 202, 1217-1228.

Hopkins, C. D., Comfort, N. C., Bastian, J., Bass, A. H., 1990. Functional analysis of sexual dimorphism in an electric fish, Hypopomus pinnicaudatus, order Gymnotiformes. Brain Behav. Evolut. 35, 350-67.

Jakob, E. M., Marshall, S. D., Uetz, G. W., 1996. Estimating fitness: a comparison of body condition indices. Oikos. 77, 61-67.

Karubian, J., Lindsay, W. R., Schwabl, H., Webster, M. S., 2011. Bill coloration, a flexible signal in a tropical passerine bird, is regulated by social environment and androgens. Anim. Behav. 81, 795-800.

Kempenaers, B., Peters, A., Foerster, K., 2008. Sources of individual variation in plasma testosterone levels. Philos. Trans. R. Soc. Lond. B. 363, 1711-23. 
Markham, M., Allee, S., Goldina, A., Stoddard, P., 2009. Melanocortins regulate the electric waveforms of gymnotiform electric fish. Horm. Behav. 55, 306-313.

Markham, M. R., Stoddard, P. K., 2005. Adrenocorticotropic hormone enhances the masculinity of an electric communication signal by modulating the waveform and timing of action potentials within individual cells. J. Neurosci. 25, 8746-8754.

Moore, F. L., Boyd, S. K., Kelley, D. B., 2005. Historical perspective: Hormonal regulation of behaviors in amphibians. Horm. Behav. 48, 373-83.

Moore, I. T., Jessop, T. S., 2003. Stress, reproduction, and adrenocortical modulation in amphibians and reptiles. Horm. Behav. 43, 39-47.

Pouso, P., Quintana, L., Bolatto, C., Silva, A. C., 2010. Brain androgen receptor expression correlates with seasonal changes in the behavior of a weakly electric fish, Brachyhypopomus gauderio. Horm. Behav. 58, 729-36.

Remage-Healey, L., Bass, A. H., 2004. Rapid, hierarchical modulation of vocal patterning by steroid hormones. J. Neurosci. 24, 5892-5900.

Remage-Healey, L., Bass, A. H., 2007. Plasticity in brain sexuality is revealed by the rapid actions of steroid hormones. J. Neurosci. 27, 1114-1122.

Salazar, V. K., The effect of male-male competition and its underlying regulatory mechanisms on the electric signal of the gymnotiform fish Brachyhypopomus gauderio., Biological Sciences. Florida International University, Miami, 2009, pp. 164.

Salazar, V. L., Stoddard, P. K., 2008. Sex differences in energetic costs explain sexual dimorphism in the circadian rhythm modulation of the electrocommunication signal of the gymnotiform fish Brachyhypopomus pinnicaudatus. J. Exp. Biol. 211, 1012-1020.

Salazar, V. L., Stoddard, P. K., 2009. Social competition affect electric signal plasticity and steroid hormone levels in the gymnotiform fish Brachyhypopomus gauderio. Horm. Behav. 56, 399-409.

Sapolsky, R. M., 1982. The endocrine stress-response and social status in the wild baboon. Horm. Behav. 16, 279-292. 
Silva, A., Quintana, L., Ardanaz, J. L., Macadar, O., 2002. Environmental and hormonal influences upon EOD waveform in gymnotiform pulse fish. J. Physiology-Paris. $96,473-484$.

Silva, A., Zubizarreta, L., Costa, G., Interspecific differences in agonistic behavior and its serotonergic modulation. International Congress of Neuroethology, Salamanca, Spain, 2010.

Stoddard, P. K., 2007, Plasticity of the electric organ discharge waveform: contexts, mechanisms, and implications for electrocommunication. In: F. Ladich, S. P. Collin, P. Moller, B. G. Kapoor, (Eds.), Communication in Fishes. Science Publisher, Inc., Enfield, NH, USA, pp. 623-646.

Stoddard, P. K., Markham, M. R., 2008. Signal cloaking by electric fish. BioScience. 58, 415-425.

Stoddard, P. K., Markham, M. R., Salazar, V. L., 2003. Serotonin modulates the electric waveform of the gymnotiform electric fish Brachyhypopomus pinnicaudatus. J. Exp. Biol. 206, 1353-1362.

Stoddard, P. K., Markham, M. R., Salazar, V. L., Allee, S., 2007. Circadian rhythms in electric waveform structure and rate in the electric fish Brachyhypopomus pinnicaudatus. Physiol. Behav. 90, 11-20.

Stoddard, P. K., Rasnow, B., Assad, C., 1999. Electric organ discharges of the gymnotiform fishes: III. Brachyhypopomus. J. Comp. Physiol. A. 184, 609-630.

Stoddard, P. K., Zakon, H. H., Markham, M. R., McAnelly, L., 2006. Regulation and modulation of electric waveforms in gymnotiform electric fish. J. Comp. Physiol. A. $192,613-624$.

Wingfield, J., Hegner, R. E., Dufty Jr, A. M., Ball, G., 1990. The "challenge hypothesis": theoretical implications for patterns of testosterone secretion, mating systems, and breeding strategies. Am. Nat. 136, 829-846.

Wingfield, J. C., Sapolsky, R. M., 2003. Reproduction and resistance to stress: when and how. J. Neuroendocrinol. 15, 711-724. 


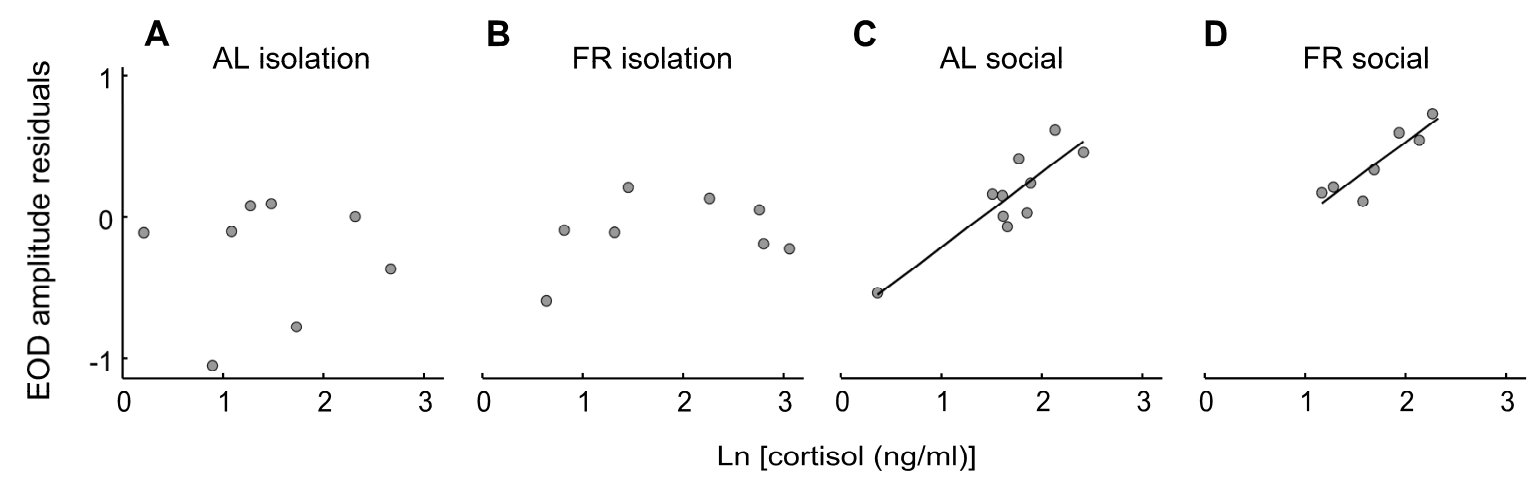

Figure 7.1. Cortisol versus EOD amplitude corrected for body length. (A-B) Cortisol levels were not related to EOD amplitude in the isolation treatments. Cortisol levels correlated with EOD amplitude only in the social treatments (C-D), regardless of food supply. However, it is unlikely that cortisol is promoting EOD amplitude since cortisol administration decreases EOD amplitude (Chapter 5). 
VITA

\section{SAT GAVASSA BECERRA}

April 8, 1982

2001-2006

2002

2003

2004-2005

2006-2007

2007-2009

2008-2009

2009
Born, Bucaramanga, Colombia

B.A., Biology

Universidad de los Andes

Bogota, Colombia

Teaching Assistant

Vertebrate Anatomy Lab

Universidad de los Andes

Bogota, Colombia

Teaching Assistant

Invertebrate Biology Lab

Universidad de los Andes

Bogota, Colombia

Teaching Assistant

Plant Physiology Lab

Universidad de los Andes

Bogota, Colombia

Teaching Assistant

General Biology Lab I \& II

Florida International University

Miami, FL

QBIC Teaching Assistant

Freshmen Journal Club

Florida International University

Miami, FL

QBIC Teaching Assistant

QBSS Summer

Florida International University Miami, FL

QBIC Teaching Assistant

Statistical Modeling

Florida International University Miami, FL 
2009-2010

2010-2011

2012
Dissertation Evidence Acquisition Fellow

Florida International University

Miami, FL

Research Assistant

P.I. Dr. Philip K. Stoddard

Florida International University

Miami, FL

Dissertation Year Fellow

Florida International University

Miami, FL

\section{PUBLICATIONS}

Gavassa, S., Roach, J. P., Stoddard, P. K., (submitted). Social regulation of electric signal plasticity in male Brachyhypopomus gauderio. J. Comp. Physiol. A.

Gavassa, S., Stoddard, P. K., (in press). Food restriction promotes signaling effort in response to social challenge in a short-lived electric fish. Hormones and Behavior.

Gavassa, S., Silva, A., Gonzalez, E., Molina, J. \& Stoddard, P. K. 2012 Social competition affects the electric communication signal of female Brachyhypopomus gauderio through androgen action. Behavioral Ecology and Sociobiology. 66, 1057-1066.

Gavassa, S., Silva, A., Gonzalez, E. \& Stoddard, P. K. 2012. Signal modulation as a mechanism for handicap disposal. Animal Behaviour. 83, 935-944

Gavassa, S., Silva, A., Stoddard, P. K., 2011. Tight hormonal phenotypic integration ensures honesty of the electric signal of male and female Brachyhypopomus gauderio. Hormones and Behavior. 60, 420-426.

Goldina, A., Gavassa, S., Stoddard, P. K., 2011. Testosterone and 11-ketotestosteone have different regulatory effects on electric communication signals of male Brachyhypopomus gauderio. Hormones and Behavior. 60, 139-147.

Weeks, O. I., Villamor, E., Tracey, M., Stoddard, P. K., Shapiro, S., Makemson, J., Garcia, R., Gavassa, S., Philippi, T., Pitzer, T., Dewsbury, D., Narasimhan, G., McGoron, A., Tashakkori, A., 2011. QBIC, an interdisciplinary and quantitative biological sciences curriculum: concept to implementation. Science Education. 12, 11-14. 